\title{
Diagnostics and mechanisms of hemostasis
}

Citation for published version (APA):

Heubel-Moenen, F. C. J. I. (2022). Diagnostics and mechanisms of hemostasis: In patients with mild bleeding disorders and thrombocytopenia. [Doctoral Thesis, Maastricht University]. ProefschriftMaken. https://doi.org/10.26481/dis.20220131fm

Document status and date:

Published: 01/01/2022

DOI:

10.26481/dis.20220131fm

Document Version:

Publisher's PDF, also known as Version of record

\section{Please check the document version of this publication:}

- A submitted manuscript is the version of the article upon submission and before peer-review. There can be important differences between the submitted version and the official published version of record.

People interested in the research are advised to contact the author for the final version of the publication, or visit the DOI to the publisher's website.

- The final author version and the galley proof are versions of the publication after peer review.

- The final published version features the final layout of the paper including the volume, issue and page numbers.

Link to publication

\footnotetext{
General rights rights.

- You may freely distribute the URL identifying the publication in the public portal. please follow below link for the End User Agreement:

www.umlib.nl/taverne-license

Take down policy

If you believe that this document breaches copyright please contact us at:

repository@maastrichtuniversity.nl

providing details and we will investigate your claim.
}

Copyright and moral rights for the publications made accessible in the public portal are retained by the authors and/or other copyright owners and it is a condition of accessing publications that users recognise and abide by the legal requirements associated with these

- Users may download and print one copy of any publication from the public portal for the purpose of private study or research.

- You may not further distribute the material or use it for any profit-making activity or commercial gain

If the publication is distributed under the terms of Article $25 \mathrm{fa}$ of the Dutch Copyright Act, indicated by the "Taverne" license above, 


\section{Diagnostics and Mechanisms of}

Hemostasis

In patients with mild bleeding disorders and thrombocytopenia 
CC Floor Heubel-Moenen, Maastricht 2022

No part of this book may be reproduced or transmitted in any form or by any means without permission by the author, or when appropriate, by the publishers of the publications.

Cover design: Jean Scheijen | vierdrie.nl

Layout: Tiny Wouters-Lenssen

Production: ProefschriftMaken | www.proefschriftmaken.nl

ISBN: 978-94-6423-603-3

Financial support by Bayer for the ProBe-AHP study, and by IFS Maastricht, Bayer, CSL Behring, Octapharma and Sobi for the production and publication of this thesis, is gratefully acknowledged. 


\section{Diagnostics and Mechanisms of Hemostasis}

\section{In patients with mild bleeding disorders and thrombocytopenia}

PROEFSCHRIFT

ter verkrijging van de graad van doctor aan de Universiteit Maastricht, op gezag van de Rector Magnificus, Prof. dr. Rianne M. Letschert volgens het besluit van het College van Decanen,

in het openbaar te verdedigen op maandag 31.01.2022 om 13.00 uur

$$
\text { door }
$$

Floortje Catharina Josephina Irene Heubel-Moenen 


\section{Promotores}

Prof. dr. E.A.M. Beckers

Prof. dr. ir. Y.M.C. Henskens

\section{Beoordelingscommissie}

Prof. dr. H. ten Cate (voorzitter)

Dr. J.M.E.M. Cosemans

Prof. dr. C.D. Dirksen

Prof. dr. M.P.M. de Maat (Erasmus Medisch Centrum Rotterdam)

Prof. dr. J.J. Zwaginga (Leids Universitair Medisch Centrum) 


\section{Contents}

Chapter 1 Introduction and outline of this thesis 7

$\begin{array}{lll}\text { PART I Diagnosis of mild bleeding disorders } & 25\end{array}$

Chapter 2 The diagnostic accuracy of bleeding assessment tools 27 for the identification of patients with mild bleeding disorders: a systematic review

Chapter 3 Screening for platelet function disorders with Multiplate and platelet function analyzer

Chapter 4 Multiparameter platelet function analysis of patients with a

prolonged platelet function analyzer closure time

Chapter 5 Effectiveness and costs of a stepwise versus an all-in-one approach 109 to diagnose mild bleeding disorders

PART II Mechanisms of bleeding in chemotherapy induced thrombocytopenia

Chapter 6 Impaired mitochondrial activity explains platelet dysfunction in

Chapter 7 Fibrinolysis in patients with chemotherapy induced thrombocytopenia and the effect of platelet transfusion

Chapter 8 General discussion and future perspectives

Addendum Samenvatting

Impact paragraph

Dankwoord

Curriculum vitae 


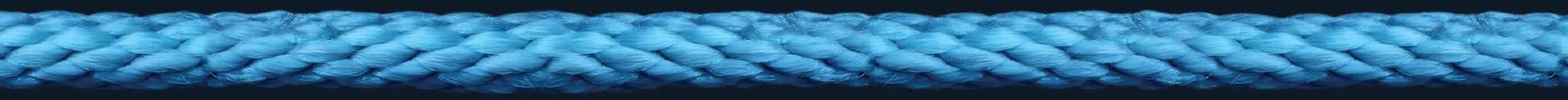




\section{CHAPTER 1}

\section{General introduction and outline of this thesis}

Based on:

Floor C.J.I. Moenen, Minka J.A. Vries, Yvonne M.C. Henskens, Hugo ten Cate, Rick J.H. Wetzels, Paul W.M. Verhezen, Rene G.M. van Oerle, Erik A.M. Beckers. Uitdagingen bij het screenen en diagnosticeren van milde bloedingsziekten.

Ned Tijdschr Hematol 2016; 13: 182-9 



\section{Hemostasis}

Hemostasis is the process of blood clot formation at the site of vessel injury and is a dynamic, highly interwoven complex of multiple processes ${ }^{1}$. For adequate hemostasis the formation and sustainability of a stable clot containing platelets, fibrinogen, red blood cells and fibrin are essential ${ }^{2}$. When a vessel wall is damaged, subendothelial collagen is exposed (Figure 1.1). Von Willebrand factor (VWF) binds to collagen and uncoils in adhesive strings. In uncoiled VWF, the binding site for glycoprotein Iba (GPIb $\alpha$ ) becomes exposed and this allows the binding of platelets to VWF ${ }^{3}$. In addition, the platelet collagen receptor (GPla/Ila) also binds to collagen. This process is called platelet adhesion. Subsequently platelets are activated.

Activated platelets secrete proteins from their granules, like adenosine diphosphate (ADP), serotonin, fibronectin, fibrinogen and thromboxane $A_{2}\left(T X A_{2}\right)$. These proteins stimulate and recruit additional platelets. The platelet integrin $\alpha_{11 b} \beta_{3}$ (GPIIB/IIIA) is exposed on the platelet surface and conformational changes lead to binding of VWF and fibrinogen, resulting in more aggregation and platelet to platelet cohesion. Activated platelets undergo shape changes, producing elongated pseudopods that make them extremely adhesive. The primary platelet plug is formed $^{3}$. Finally, conformational changes of the platelet membrane, the so called flipflop mechanism, lead to the exposure of procoagulant phospholipids, mainly phosphatidylserine (PS), providing a procoagulant surface for the binding of clotting factor complexes ${ }^{2}$.

Next, the coagulation cascade is activated (Figure 1.2), leading to the production of thrombin which turns fibrinogen into fibrin, to stabilize the platelet plug. Classically, the coagulation cascade is described as it occurs in vitro. However a newer model, describing the coagulation cascade in vivo, is widely accepted ${ }^{4}$.

In vitro, two main pathways initiate and amplify the coagulation cascade: the extrinsic and intrinsic pathway. The intrinsic (contact activation) pathway is initiated when blood comes into contact with negatively charged surfaces leading to autoactivation of factor XII (FXII). This results in the generation of small amounts of activated FXII (FXIla). FXIla cleaves prekallikrein to kallikrein, which leads to a subsequent activation of FXI and FIX. Factor IXa, in complex with FVIIIa and FX forms the intrinsic tenase complex. The extrinsic pathway is activated when tissue factor (TF), located on damaged subendothelial cells binds and activates FVII circulating in blood, and together with FX the extrinsic tenase complex is formed ${ }^{5}$. Both the intrinsic and extrinsic tenase complexes induce activation of FX. Factor $\mathrm{Xa}$, FVa and calcium form the prothrombinase complex which leads to activation of FII, by converting prothrombin into thrombin. Thrombin turns fibrinogen into fibrin ${ }^{2}$. 
Figure 1.1 Primary hemostasis.

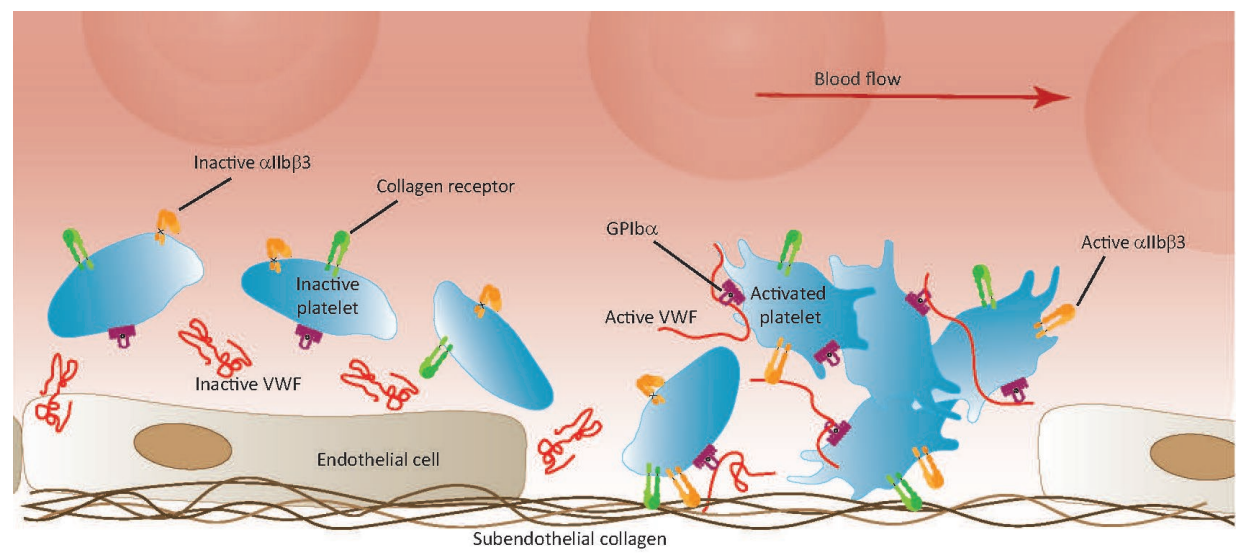

With vascular damage and exposure of subendothelial collagen, VWF binds to collagen and uncoils in adhesive strings allowing the binding of platelet GPIba. In addition, the platelet collagen receptors bind to collagen. The platelets adhere and are activated, leading to a conformational change in platelet integrin $\alpha$ llb $\beta 3$. The activated platelets become irregularly shaped. VWF and fibrinogen bind to the activated integrin $\alpha$ llb $\beta 3$, leading to aggregation of the activated platelets ${ }^{3}$.

In vivo, the coagulation process starts with an initiation phase with TF, as the key initiator of the coagulation cascade, binding to FVIIa ${ }^{4}$. This TF/FVIla complex activates small amounts of FIX and FX and the prothrombinase complex (FXa/FV) is formed, which converts prothrombin into thrombin. In the amplification phase, the slowly accumulating thrombin activates FIX and cofactor FVIII, and this greatly enhances the catalytic activity of FIX and FX leading to formation of large amounts of thrombin ('thrombin burst'), resulting in fibrin deposition'. To prevent excessive fibrin formation, coagulation is regulated by the fibrinolytic system and coagulation inhibitors. In the fibrinolytic pathway, tissue plasminogen activator (tPA) converts plasminogen to plasmin, which in turn cleaves fibrin into fibrin degradation products $(F D P)^{6}$. Fibrinolysis is inhibited by $\alpha_{2}$-antiplasmin $\left(\alpha_{2}-A P\right)$, plasminogen activator inhibitor-1 (PAI-1), PAI-2, and attenuated by thrombin-activatable fibrinolysis inhibitor (TAFI). TAFI decreases plasminogen binding to fibrin. Abnormal bleeding may occur when specific elements of these processes are missing or dysfunctional. 
Figure 1.2 Coagulation cascade.

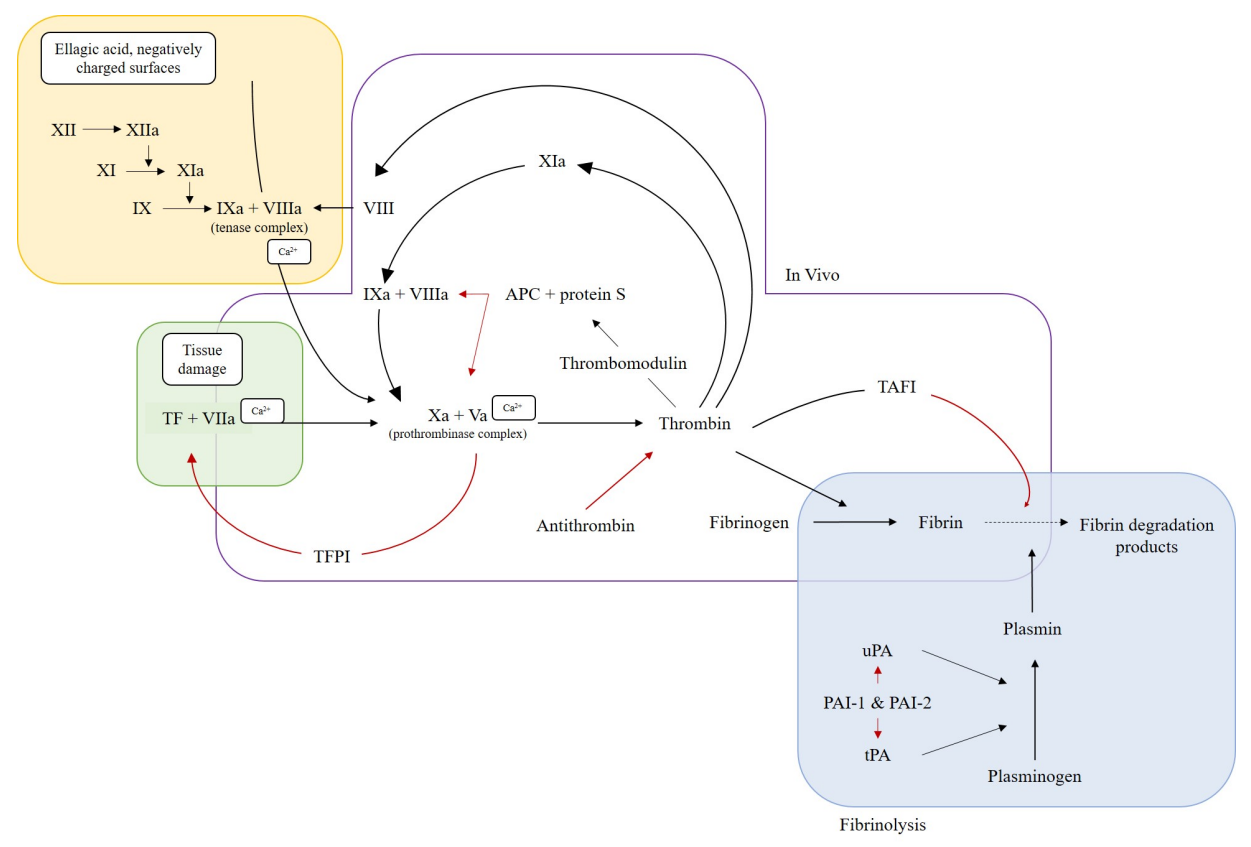

Coagulation cascade: black arrows indicate activation, red arrows indicate inhibition. Abbreviations: a, activated; $\alpha 2$-AP, $\alpha 2$-antiplasmin; Ca, calcium; TAFI, thrombin-activatable fibrinolysis inhibitor; TF, tissue factor; TFPI, tissue factor plasminogen inhibitor; PAI, plasminogen activator inhibitor; tPA, tissue plasminogen activator.

\section{Part I. Diagnosis of patients with mild bleeding disorders}

\section{Mild bleeding disorders}

Mild bleeding disorders (MBD) are a heterogeneous group including von Willebrand disease (VWD), platelet function disorders (PFD), mild clotting factor deficiencies, fibrinolytic abnormalities or a combination of defects ${ }^{7}$. The bleeding tendency in patients with mild bleeding disorders is, in addition to spontaneous mucosal bleeding or hematomas, mainly manifested by disproportionate bleeding after minor injuries, surgery and childbirth ${ }^{8}$.

Although patients with mild bleeding disorders do not have an increased mortality, they may bleed after surgery requiring blood products, re-surgery, or hemostatic agents to control the bleeding ${ }^{9}$. Frequently, an unexpected perioperative hemorrhage is the first presentation of a mild bleeding disorder and often the main reason to perform diagnostic work-up for a bleeding disorder ${ }^{10,11}$. 


\section{Diagnostic challenges in mild bleeding disorders}

Patients with a severe bleeding disorder often have clear symptoms which, in the case of a congenital disease, are expressed early in life. In patients with a mild bleeding disorder, the clinical symptoms are very heterogeneous and present later in life, or only after surgery. Also, in severe bleeding disorders, laboratory test abnormalities are obvious, reproducible and with a straight forward interpretation. However, in patients with a mild bleeding disorder, laboratory test results can be unclear or inconsistent, and the interpretation is often debatable ${ }^{12}$. Furthermore, in mild bleeding disorders, there is often no good correlation between the level of coagulation factor and the bleeding symptoms ${ }^{13,14}$. Many diagnostic algorithms have been proposed, giving guidance to a stepwise analysis of patients referred for bleeding evaluation ${ }^{15-17}$.

\section{Bleeding history}

Due to the high prevalence of trivial and minor bleeding symptoms in the general population, the interpretation of bleeding symptoms in patients with a suspected bleeding disorder can be challenging. As a result, mild bleeding can be considered normal, while trivial bleeding sometimes leads to extensive and unnecessary investigations ${ }^{18}$. Bleeding symptoms such as menorrhagia (47\%), epistaxis (25\%), easy bruising (18\%), and prolonged bleeding after dental extraction (18\%) are frequently reported in the healthy population ${ }^{19}$. The reporting of bleeding symptoms is also influenced by socioeconomic status, family history and personality ${ }^{20}$. Older individuals are less likely to report bleeding symptoms from the past, and if there is a positive family history, abnormal bleeding symptoms may be considered normal. On the other hand, a lot of attention might be given to trivial bleeding. Finally, there is no specific bleeding profile that discriminates between the different mild bleeding disorders ${ }^{8}$.

In order to distinguish patients with bleeding symptoms indicating an underlying bleeding disorder from healthy subjects with trivial bleeding symptoms, many attempts have been made to standardize and validate tools to report and interpret the bleeding history ${ }^{21-29}$. This has led to the development of the so-called 'bleeding assessment tools' (BAT), consisting of a standardized bleeding questionnaire and a computed interpretation score. The purpose of these questionnaires is to structurally question and display bleeding symptoms and to contribute to the diagnosis of mild bleeding disorders ${ }^{30-33}$. 


\section{Hemostatic tests}

Several studies show that, even after extensive laboratory testing, in $50-75 \%$ of patients referred for bleeding evaluation, no bleeding disorder is found ${ }^{34-36}$. Still, if these patients have a relevant bleeding history, they are often provided with a treatment plan to prevent bleeding during surgery or childbirth ${ }^{34,35,37}$.

The hemostatic laboratory assays can be classified into screening tests, confirmatory ('gold standard') tests and experimental assays. Screening tests commonly used to detect clotting factor deficiencies are prothrombin time (PT) and activated partial thromboplastin time (aPTT). These are sensitive enough to detect serious deficiencies (coagulation factor level $<30 \%$ ), but they are not suitable for detecting mild deficiencies or factor XIII deficiency ${ }^{16}$.

The platelet function analyzer-200 (PFA-200) is a screening test for VWD and platelet function disorders. It has a high sensitivity for VWD (VWF $<30 \%$ ), and severe platelet function disorders such as Bernard Soulier or Glanzmann thrombasthenia (sensitivity: 62-100\%), but not for mild VWD (VWF 30-50\%) or mild platelet function disorders (sensitivity: $24-58 \%$ ) $^{38,39}$. The diagnosis of VWD is mainly based on the level of VWF. However, the normal range of VWF is wide, $95 \%$ of the values fall within $50 \%$ and $200 \%$. In addition, VWF increases with physical activity, stress, infection, age and in pregnancy ${ }^{40-42}$, while blood group $\mathrm{O}$ is associated with lower VWF levels ${ }^{43}$.

Light transmission aggregometry (LTA) is the gold standard for platelet function disorders ${ }^{44}$. The LTA shows very clear deviations in Glanzmann thrombasthenia (GT) or Bernard Soulier (BSS). However, mild platelet defects are more difficult to detect, because they only exhibit mild or combined defects in platelet aggregation and secretion ${ }^{45}$.

Fibrinolysis proteins like PAI and $\alpha 2$-AP can be measured in order to investigate for fibrinolysis disorders ${ }^{46}$. The Euglobin clotlysis test is a global screening test to display fibrinolysis ${ }^{47}$. However, the value of this test in diagnosing patients with increased fibrinolytic activity is unknown ${ }^{16}$.

Finally, when interpreting the results of these laboratory tests, pre-analytical conditions and pitfalls ${ }^{48,49}$, standardization issues of different test methods ${ }^{50}$ and the effect of patient characteristics and behavior on test results ${ }^{51,52}$ should be taken into account.

\section{New hemostatic tests}

In the past years, new laboratory tests have emerged that claim to be able to discriminate normal from abnormal hemostasis. In these new hemostatic assays the test methods and designs have shifted from: slow and time consuming tests to pointof-care tests ${ }^{53}$; from testing a specific coagulation factor to testing more global 
hemostatic function ${ }^{53,54}$; from plasma based testing to whole blood testing, taking into account the interaction of platelets, red blood cells and leukocytes ${ }^{55,56}$, and from a stirring/static test method to flow based testing ${ }^{57,58}$.

Examples of new tests are: whole blood impedance platelet aggregation (Multiplate $^{59}$, whole blood rotational thromboelastography (ROTEM or TEG) with various agonists including tPA-ROTEM ${ }^{56,60}$, thrombin generation ${ }^{61}$, multiparameter microfluidic assays ${ }^{62}$ and flowcytometry ${ }^{63}$. In addition, there is an increasing focus on genetic testing like whole exome sequencing (WES ${ }^{64,65}$. However, the value of all these new tests in structurally identifying patients with a bleeding disorder or in supporting the treatment of these patients has yet to be determined.

\section{Predictors of bleeding}

The project 'Predictors of Bleeding' of the Maastricht University Medical Center+ (MUMC+), housed at the Cardiovascular Research Institute Maastricht (CARIM) research school, is an initiative of the laboratory for Hemostasis and Transfusion (Central Diagnostic Laboratory, MUMC+), the clinical department of Hematology/Internal Medicine and the faculty department of Biochemistry. This project contains various lines of research on bleeding tendency in different patient populations, including preoperative patients (PANE study), patients referred for bleeding evaluation (ProBe-AHP study), and patients with a known bleeding disorder (BEPA study) (Figure 1.3). In addition, there is a close collaboration with the Ouwehand laboratory of the Hematology Department of the University of Cambridge, with participation in the ThromboGenomics project (TG) and the BRIDGE Bleeding Thrombotic and Platelet Disorders project ${ }^{66}$. Within these projects, a genetic package (the ThromboGenomics HTS gene panel test) is being developed to make a rapid (genetic) diagnosis in patients who present with a bleeding tendency ${ }^{64}$.

In all patients participating in the PANE, ProBe-AHP and BEPA studies, the International Society on Thrombosis and Haemostasis Bleeding Assessment Tool (ISTH BAT), all available routine laboratory coagulation tests, and a number of experimental research tests, including the ThromboGenomics HTS gene panel test, will be performed (Table 1.1). The studies are described in short below, a detailed description of the study designs is presented in Appendix 3.A. The ultimate goal of the studies in the project 'Predictors of Bleeding', is to improve the diagnostic work-up in patients with a bleeding tendency, to determine the value and place of new diagnostic tests, including DNA testing, and to gain more inside in mechanisms of bleeding in different patient populations. This will finally lead to the development of a new bleeding algorithm: the Bleeding Algorithm Maastricht (BAM). 
Figure 1.3 Research lines in the project 'Predictors of Bleeding'.

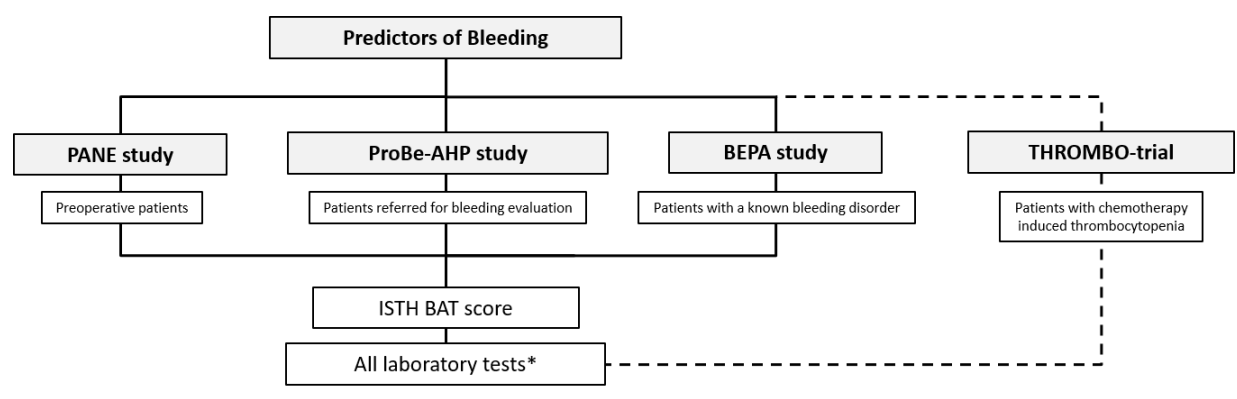

* See Table 1.1. BAT, bleeding assessment tool; ISTH, International Society on Thrombosis and Haemostasis.

Verification of preoperative screening for bleeding tendency and per-operative bleeding risk management, the PANE study

From September 2013 until January 2016, preoperative patients who indicated one or more bleeding symptoms on the anesthetist's preoperative screening list were included in the PANE study. Preoperative patients without bleeding symptoms were included as a control group. In all patients the ISTH BAT was performed together with full study laboratory examination. The aim of this study was to determine the percentage of pre-operative patients with a hemostatic abnormality. In addition, the value of an extensive hemostatic laboratory examination and the ISTH BAT for identifying patients with a high surgical bleeding risk was investigated ${ }^{10}$.

\section{Predictors of Bleeding Evaluation in Adult Hematologic Patients with Bleeding Tendencies, the ProBe-AHP (Bloody) study}

Since 2015, patients who are referred to the hematology clinic for bleeding evaluation, have been included in the ProBe-AHP (Bloody) study. In all patients the ISTH BAT is performed together with full study laboratory examination. Depending on the bleeding score, the results of the screening and/or conventional laboratory tests are visible to the treating physician. The final diagnosis of the patient is decided on in a multidisciplinary team in the presence of a senior hematologist, a clinical chemist and a senior analyst. The aim of the study is to see whether or not experimental hemostatic tests detect abnormalities in patients with a certain diagnosis and to investigate the value of these tests in the diagnostic work-up of patients with mild bleeding disorders. 
Predictors of Bleeding Evaluation in Adult Hematologic Patients with Bleeding Tendencies. Patients with established bleeding disorders: The BePa verification study

Since 2015, patients with a known bleeding disorder registered at the hemophilia treatment center MUMC+ have been approached to participate in the BEPA study. This concerns patients with hemophilia A or B, other factor deficiencies, VWD, platelet function disorders and fibrinolysis disorders. In these patients, the ISTH BAT is performed together with full study laboratory examination, if applicable before and after hemostatic medication. The aim of this study is to gain more insight into hemostatic mechanisms in certain bleeding disorders and to investigate whether or not the experimental hemostatic tests are able to detect bleeding disorders and to investigate their contribution to the monitoring of the treatment of these patients.

Table 1.1 Overview of hemostatic systems and laboratory tests performed in the project 'Predictors of Bleeding'.

\begin{tabular}{|c|c|c|c|c|}
\hline Tests & Coagulation/VWF & Platelet function & Fibrinolysis & Global \\
\hline Screening & PT, aPTT, TT & $\begin{array}{l}\text { Platelet count and } \\
\text { smear, MPV, PFA-200 }\end{array}$ & Euglobin clotlysis & \\
\hline $\begin{array}{l}\text { Confirmatory 'gold } \\
\text { standard' tests }\end{array}$ & $\begin{array}{l}\text { Fibrinogen, Factor II, } \\
\text { V, VII, VIII, IX, X, XI, } \\
\text { XII, XIII, VWF antigen } \\
\text { and activity } \\
\text { Single gene analysis }\end{array}$ & $\begin{array}{l}\text { Light transmission } \\
\text { aggregometry } \\
\text { Flowcytometry } \\
\text { Single gene analysis }\end{array}$ & $\begin{array}{l}\text { tPA, PAI-1, } \alpha 2- \\
\text { antiplasmin, TAFI } \\
\text { Single gene analysis }\end{array}$ & \\
\hline Experimental & ThromboGenomics & Multiplate & tPA-ROTEM & ROTEM \\
\hline research tests & HTS gene panel & $\begin{array}{l}\text { Multiparameter } \\
\text { microfluidic tests } \\
\text { Flowcytometry } \\
\text { ThromboGenomics } \\
\text { HTS gene panel }\end{array}$ & $\begin{array}{l}\text { Plasmin generation } \\
\text { ThromboGenomics } \\
\text { HTS gene panel }\end{array}$ & $\begin{array}{l}\text { Thrombin } \\
\text { generation } \\
\text { ThromboGenomics } \\
\text { HTS gene panel }\end{array}$ \\
\hline
\end{tabular}

aPTT, activated partial thrombin time; LTA, light transmission aggregometry; MPV, mean platelet volume; PAI-1, plasminogen activator inhibitor-1; PFA, platelet function analyzer; PT, prothrombin time; ROTEM, rotational thromboelastometry; TAFI, thrombin activatable fibrinolysis inhibitor; tPA, tissue plasminogen activator; TT, thrombin time; VWF, von Willebrand Factor.

\section{Part II Bleeding risk in patients with chemotherapy induced thrombocytopenia}

Platelet transfusions are widely used to prevent and treat bleeding in thrombocytopenic patients ${ }^{67,68}$. More than 4.4 million platelet products are transfused in the USA and Europe each year ${ }^{68}$, of which up to $67 \%$ are issued to patients with hematologic malignancies ${ }^{69}$. In patients with hematologic malignancies, severe thrombocytopenia ( $\left.<50 \times 10^{9} / \mathrm{L}\right)$ frequently develops as a consequence of the disease or its treatment and is associated with an increased risk of bleeding ${ }^{69,70}$. These 
patients are given prophylactic platelet transfusions when platelet count falls below $10 \times 10^{9} / L^{68,71}$. However, the routine use of prophylactic platelet transfusion has been debated, mainly because the effectiveness of platelet transfusions to prevent bleeding in patients with hematologic malignancies remains unclear ${ }^{70}$. Also, platelet transfusion is associated with serious complications, like transfusion related acute lung injury ${ }^{72}$.

Researchers have compared a therapeutic-only policy with the usual prophylactic platelet transfusion policy and found that, despite prophylaxis, a significant number of patients still had a bleeding, and a significant number of patients who did not receive prophylactic transfusions, did not bleed despite thrombocytopenia ${ }^{70,73,74}$. These observations indicate that the risk of bleeding during a thrombocytopenic episode does not solely depend on platelet count, and other factors of hemostasis or the vessel wall might contribute significantly to bleeding or protection against bleeding. A number of other factors have already been associated with bleeding, like low hematocrit, type of disease, recent allogeneic stem cell transplantation (SCT), infection, splenomegaly and previous bleeding ${ }^{69,75-77}$. In 2018, the American Society of Clinical Oncology guideline for platelet transfusion for patients with cancer recommended a more personalized transfusion strategy ${ }^{78}$, however, the knowledge on additional risk factors for bleeding has not yet led to a change in the currently applied prophylactic transfusion strategy ${ }^{79}$.

THROMBO-trial: validation of a new fibrinolysis assay for thromboelastometry on the ROTEM device: the effects of platelet transfusions on hemostasis parameters in patients with hematological malignancies.

The THROMBO-trail is another research line in the 'Predictors of Bleeding' project of the MUMC +. The aim of this study is to quantify the effect of platelet transfusion on hemostatic parameters in hemato-oncological patients with chemotherapy-induced thrombocytopenia and to identify other determinants of hemostasis which contribute to clot formation and clot strength. Adult patients undergoing intensive chemotherapy for whom prophylactic platelet transfusions are indicated, are eligible to participate in the study. In all patients, bleeding events will be recorded and preand post-platelet transfusion hemostatic tests will be performed as depicted in Table 1.1, with the exception of PFA-200, LTA, Multiplate and DNA analysis. 


\section{Outline of this thesis}

This thesis focuses on the value of several diagnostic tests and diagnostic approaches in patients referred for bleeding evaluation (Part I) and on clarifying mechanisms of bleeding in patients with chemotherapy induced thrombocytopenia (Part II).

The introductory Chapter 1 provides background information and describes the different research lines in the project 'Predictors of Bleeding'. In Chapter 2 a systematic review of diagnostic studies assessing the performance of the bleeding assessment tool in patients referred for evaluation of bleeding symptoms is described. In Chapter 3 the diagnostic value of the Multiplate and PFA regarding detection of platelet function abnormalities in different patient populations is assessed. Chapter 4 describes multiparameter microfluidic platelet function analysis in bleeding patients with a prolonged platelet function analyzer closure time as the only aberrant finding in their diagnostic work-up. In Chapter 5 effectiveness, healthcare resource use and costs, and patient burden of two diagnostic approaches for patients referred for bleeding evaluation are compared.

Chapter 6 describes the platelet activation processes and procoagulant activity in hemato-oncological patients with chemotherapy induced thrombocytopenia (CIT) and describes the effects of platelet transfusion. In Chapter 7 the fibrinolytic potential of whole blood clots of CIT patients is explored with TPA-ROTEM, and the effect of platelet transfusion is described. Chapter 8 provides an overview of the most important findings of this thesis, leading to the formation of a number of statements and topics which will be discussed in this chapter. Implications for both clinical practice and future research are discussed. 


\section{References}

1. Furie B, Furie BC. Mechanisms of thrombus formation. N Engl J Med 2008;359(9):938-49.

2. Versteeg $\mathrm{HH}$, et al. New fundamentals in hemostasis. Physiol Rev 2013;93(1):327-58.

3. Leebeek FW, Eikenboom JC. Von Willebrand's Disease. N Engl J Med 2016;375(21):2067-80.

4. Monroe DM, Hoffman M. What does it take to make the perfect clot? Arterioscler Thromb Vasc Biol 2006;26(1):41-8.

5. Smith SA, Travers RJ, Morrissey JH. How it all starts: Initiation of the clotting cascade. Crit Rev Biochem Mol Biol 2015;50(4):326-36.

6. Chapin JC, Hajjar KA. Fibrinolysis and the control of blood coagulation. Blood Rev 2015;29(1):17-24.

7. Mezzano D, Quiroga T. Diagnostic challenges of inherited mild bleeding disorders: a bait for poorly explored clinical and basic research. J Thromb Haemost 2019;17(2):257-70.

8. Quiroga T, Mezzano D. Is my patient a bleeder? A diagnostic framework for mild bleeding disorders. Hematology Am Soc Hematol Educ Program 2012;2012:466-74.

9. Orsini $\mathrm{S}$, et al. Bleeding risk of surgery and its prevention in patients with inherited platelet disorders. Haematologica 2017;102(7):1192-203.

10. Vries MJ, et al. Preoperative screening for bleeding disorders: A comprehensive laboratory assessment of clinical practice. Res Pract Thromb Haemost 2018;2(4):767-77.

11. Kuiper $\mathrm{G}$, et al. Routine haemostasis testing before transplanted kidney biopsy: a cohort study. Transpl Int 2018;31(3):302-12.

12. de Moerloose P, et al. Diagnosis of mild bleeding disorders. Swiss Med Wkly 2009;139(23-24):327-32.

13. Olsson A, et al. Clotting factor level is not a good predictor of bleeding in carriers of haemophilia $A$ and B. Blood Coagul Fibrinolysis 2014;25(5):471-5.

14. Rodeghiero F, Castaman G. Congenital von Willebrand disease type I: definition, phenotypes, clinical and laboratory assessment. Best Pract Res Clin Haematol 2001;14(2):321-35.

15. Boender J, Kruip MJ, Leebeek FW. A diagnostic approach to mild bleeding disorders. J Thromb Haemost 2016;14(8):1507-16.

16. Hayward CPM. How I investigate for bleeding disorders. Int J Lab Hematol 2018;40 Suppl 1:6-14.

17. Rodeghiero F, et al. Fundamentals for a Systematic Approach to Mild and Moderate Inherited Bleeding Disorders: An EHA Consensus Report. Hemasphere 2019;3(4):e286.

18. Tosetto A, Castaman G, Rodeghiero F. Bleeders, bleeding rates, and bleeding score. J Thromb Haemost 2013;11 Suppl 1:142-50.

19. Mauer AC, et al. Impact of sex, age, race, ethnicity and aspirin use on bleeding symptoms in healthy adults. J Thromb Haemost 2011;9(1):100-8.

20. Rodeghiero F, Tosetto A, Castaman G. How to estimate bleeding risk in mild bleeding disorders. J Thromb Haemost 2007;5:157-66.

21. Rodeghiero F, et al. The discriminant power of bleeding history for the diagnosis of type 1 von Willebrand disease: an international, multicenter study. J Thromb Haemost 2005;3(12):2619-26.

22. Tosetto A, et al. Prospective evaluation of the clinical utility of quantitative bleeding severity assessment in patients referred for hemostatic evaluation. J Thromb Haemost 2011;9(6):1143-8.

23. Tosetto $A$, et al. A quantitative analysis of bleeding symptoms in type 1 von Willebrand disease: results from a multicenter European study (MCMDM-1 VWD). J Thromb Haemost 2006;4(4):766-73.

24. Sramek A, et al. Usefulness of Patient Interview in Bleeding Disorders. Arch Intern Med 1995;155(13): 1409-15.

25. Azzam HA, et al. The condensed MCMDM-1 VWD bleeding questionnaire as a predictor of bleeding disorders in women with unexplained menorrhagia. Blood Coagul Fibrinolysis 2012;23(4):311-5.

26. Kaur $\mathrm{H}$, et al. Systematic analysis of bleeding phenotype in PT-VWD compared to type $2 \mathrm{~B}$ VWD using an electronic bleeding questionnaire. Clin Appl Thromb Hemost 2014;20(8):765-71.

27. Philipp CS, et al. Evaluation of a screening tool for bleeding disorders in a US multisite cohort of women with menorrhagia. Am J Obstet Gynecol 2011;204(3):209 e1-7.

28. Deforest $\mathrm{M}$, et al. Generation and optimization of the self-administered bleeding assessment tool and its validation as a screening test for von Willebrand disease. Haemophilia 2015;21(5):E384-8. 
29. Lowe GC, et al. Utility of the ISTH bleeding assessment tool in predicting platelet defects in participants with suspected inherited platelet function disorders. J Thromb Haemost 2013;11(9): 1663-8.

30. Rydz N, James PD. The evolution and value of bleeding assessment tools. J Thromb Haemost 2012; 10(11):2223-9.

31. Tosetto A, Castaman G, Rodeghiero F. Bleeding scores in inherited bleeding disorders: clinical or research tools? Haemophilia 2008;14(3):415-22.

32. Gresele P, et al. Validation of the ISTH/SSC bleeding assessment tool for inherited platelet disorders: A communication from the Platelet Physiology SSC. J Thromb Haemost 2020;18(3):732-9.

33. Gresele P, et al. The ISTH bleeding assessment tool as predictor of bleeding events in inherited platelet disorders: Communication from the ISTH SSC Subcommittee on Platelet Physiology. J Thromb Haemost 2021;19(5):1364-71.

34. Quiroga T, et al. High prevalence of bleeders of unknown cause among patients with inherited mucocutaneous bleeding. A prospective study of 280 patients and 299 controls. Haematologica 2007; 92(3):357-65.

35. Gebhart J, et al. High proportion of patients with bleeding of unknown cause in persons with a mildto-moderate bleeding tendency: Results from the Vienna Bleeding Biobank (VIBB). Haemophilia 2018;24(3):405-13.

36. Thomas W, Downes K, Desborough MJR. Bleeding of unknown cause and unclassified bleeding disorders; diagnosis, pathophysiology and management. Haemophilia 2020;26(6):946-57.

37. MacDonald S, et al. Characterization of a large cohort of patients with unclassified bleeding disorder; clinical features, management of haemostatic challenges and use of global haemostatic assessment with proposed recommendations for diagnosis and treatment. Int J Lab Hematol 2019;42(2):116-25.

38. Cattaneo $\mathrm{M}$, et al. Evaluation of the PFA-100 system in the diagnosis and therapeutic monitoring of patients with von Willebrand disease. Thromb Haemost 1999;82(1):35-9.

39. Quiroga T, et al. Template bleeding time and PFA-100 have low sensitivity to screen patients with hereditary mucocutaneous hemorrhages: comparative study in 148 patients. J Thromb Haemost 2004;2(6):892-8.

40. Chapin J, Von Willebrand disease in the elderly: clinical perspectives. Clin Interv Aging 2018;13: 1531-41.

41. van Loon JE, et al. Performance related factors are the main determinants of the von Willebrand factor response to exhaustive physical exercise. PLoS One 2014;9(3):e91687.

42. Castaman G, James PD. Pregnancy and delivery in women with von Willebrand disease. Eur J Haematol 2019;103(2):73-9.

43. Ward SE, O'Sullivan JM, O'Donnell JS. The relationship between ABO blood group, von Willebrand factor, and primary hemostasis. Blood 2020;136(25):2864-74.

44. Gresele P. Diagnosis of inherited platelet function disorders: guidance from the SSC of the ISTH. J Thromb Haemost 2015;13(2):314-22.

45. Hayward CPM, et al. Update on diagnostic testing for platelet function disorders: What is practical and useful? Int J Lab Hematol 2019;41 Suppl 1:26-32.

46. Saes JL, et al. Hemorrhagic disorders of fibrinolysis: a clinical review. J Thromb Haemost, 2018.

47. Ilich A, Bokarev I, Key NS. Global assays of fibrinolysis. Int J Lab Hematol 2017;39(5):441-7.

48. Denessen EJS, et al. Determining the Optimal Storage Time and Temperature for Performing Platelet Function Assays and Global Hemostasis Assays. Platelets 2021:1-9.

49. Denessen EJS, et al. Determining the maximal storage time of centrifuged citrated samples for performing add-on routine coagulation tests. Thromb Res 2020;196:54-62.

50. Munnix ICA, et al. Harmonizing light transmission aggregometry in the Netherlands by implementation of the SSC-ISTH guideline. Platelets 2020:1-8.

51. Krekels JPM, Verhezen PWM, Henskens YMC. Platelet Aggregation in Healthy Participants is Not Affected by Smoking, Drinking Coffee, Consuming a High-Fat Meal, or Performing Physical Exercise. Clin Appl Thromb Hemost 2019;25:1076029618782445. 
52. Kuiper $\mathrm{G}$, et al. The use of regression analysis in determining reference intervals for low hematocrit and thrombocyte count in multiple electrode aggregometry and platelet function analyzer 100 testing of platelet function. Platelets 2017;28(7):668-75.

53. Ganter MT, Hofer CK. Coagulation monitoring: Current techniques and clinical use of viscoelastic point-of-care coagulation devices. Anesth Analg 2008;106(5):1366-75.

54. Kuiper GJ, Henskens YM. Rapid and Correct Prediction of Thrombocytopenia and Hypofibrinogenemia with Rotational Thromboelastometry in Cardiac Surgery Reconsidered. J Cardiothorac Vasc Anesth 2016;30(6):e55-6.

55. Vries MJA, et al. Assessment and determinants of whole blood and plasma fibrinolysis in patients with mild bleeding symptoms. Thromb Res 2019;174:88-94.

56. Kuiper GJAJM, et al. Validation of a modified thromboelastometry approach to detect changes in fibrinolytic activity. Thromb J 2016;14:1.

57. Herfs L, et al. Multiparameter Microfluidics Assay of Thrombus Formation Reveals Increased Sensitivity to Contraction and Antiplatelet Agents at Physiological Temperature. Thromb Res 2021; 203:46-56.

58. Swieringa F, et al. Platelet Control of Fibrin Distribution and Microelasticity in Thrombus Formation Under Flow. Arterioscler Thromb Vasc Biol 2016;36(4):692-9.

59. Albanyan A, et al. Diagnosis of Glanzmann thrombasthenia by whole blood impedance analyzer (MEA) vs. light transmission aggregometry. Int J Lab Hematol 2015;37(4):503-8.

60. Nair SC, et al. Tests of global haemostasis and their applications in bleeding disorders. Haemophilia 2010;16:85-92.

61. Dargaud $\mathrm{Y}$, et al. Evaluation of thrombin generating capacity in plasma from patients with haemophilia A and B. Thrombosis and Haemostasis 2005;93(3):475-80.

62. Brouns SLN, van Geffen JP, Heemskerk JWM. High-throughput measurement of human platelet aggregation under flow: application in hemostasis and beyond. Platelets 2018;29(7):662-9.

63. van Asten I, Schutgens REG, Urbanus RT. Toward Flow Cytometry Based Platelet Function Diagnostics. Semin Thromb Hemost 2018;44(3):197-205.

64. Downes K, et al. Diagnostic high-throughput sequencing of 2396 patients with bleeding, thrombotic, and platelet disorders. Blood 2019;134(23):2082-91.

65. Simeoni I, et al. A high-throughput sequencing test for diagnosing inherited bleeding, thrombotic, and platelet disorders. Blood 2016;127(23):2791-803.

66. Ouwehand, P.W.H. ThromboGenomics project (TG) and the BRIDGE Bleeding Thrombotic and Platelet Disorders project. 2020; Available from: http://www.haem.cam.ac.uk/staff/senior-staff/professorwillem-h-ouwehand/.

67. McCullough J. Overview of Platelet Transfusion. Semin Hematol 2010;47(3):235-42.

68. Stroncek DF, Rebulla P. Platelet transfusions. Lancet 2007;370(9585):427-38.

69. Estcourt LJ, et al. Prospective observational cohort study of the association between thromboelastometry, coagulation and platelet parameters and bleeding in patients with haematological malignancies- The ATHENA study. Br J Haematol 2014;166(4):581-91.

70. Stanworth SJ, et al. A no-prophylaxis platelet-transfusion strategy for hematologic cancers. N Engl J Med 2013;368(19):1771-80.

71. Gmur J, et al. Safety of stringent prophylactic platelet transfusion policy for patients with acute leukaemia. Lancet 1991;338(8777):1223-6.

72. Blumberg N, et al. Platelets - to transfuse or not to transfuse. Lancet 2012;380(9850):1287-9.

73. Wandt $\mathrm{H}$, et al. Therapeutic platelet transfusion versus routine prophylactic transfusion in patients with haematological malignancies: an open-label, multicentre, randomised study. Lancet 2012; 380(9850):1309-16.

74. Zeller MP, Al-Habsi KS, Heddle NM. Prophylactic platelet transfusions: should they be a treatment of the past? Curr Opin Hematol 2014;21(6):521-7.

75. Uhl L, et al. Laboratory predictors of bleeding and the effect of platelet and RBC transfusions on bleeding outcomes in the PLADO trial. Blood 2017;130(10):1247-58.

76. Vinholt PJ, et al. Prediction of bleeding and prophylactic platelet transfusions in cancer patients with thrombocytopenia. Platelets 2016;27(6):547-54. 
77. Friedmann AM, et al. Do basic laboratory tests or clinical observations predict bleeding in thrombocytopenic oncology patients? A reevaluation of prophylactic platelet transfusions. Transfus Med Rev 2002;16(1):34-45.

78. Schiffer CA, et al. Platelet Transfusion for Patients With Cancer: American Society of Clinical Oncology Clinical Practice Guideline Update. J Clin Oncol 2018;36(3):283-99.

79. Cornelissen LL, et al. Risk factors for bleeding in haemato-oncology patients-a nested case-control study: The BITE study protocol (Bleeding In Thrombocytopenia Explained). BMJ Open 2020;10(6): e034710. 


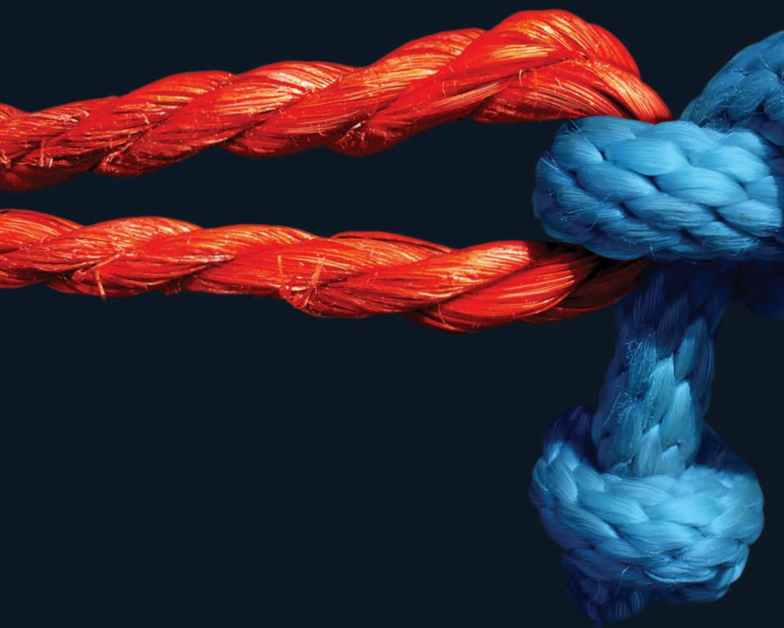


\section{PART I}

Diagnosis of mild bleeding disorders 


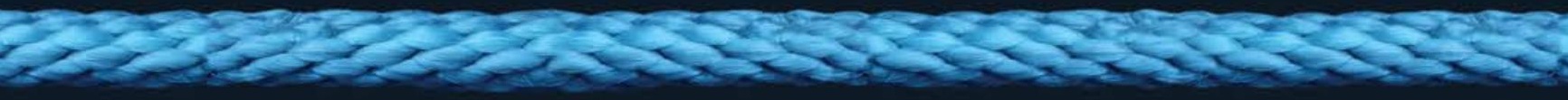




\section{CHAPTER 2}

The diagnostic accuracy of bleeding assessment tools for the identification of patients with mild bleeding disorders: a systematic review

Floor C.J.I Moenen, Patricia J. Nelemans, Saskia E.M. Schols, Harry C. Schouten, Yvonne. M.C. Henskens, Erik A.M. Beckers

Haemophilia. 2018 Jul;24(4):525-535 


\section{Abstract}

\section{Introduction}

The bleeding assessment tool (BAT) has been developed to standardize and interpret bleeding history in patients evaluated for a bleeding disorder. A critical appraisal addressing the quality and results of validation studies is lacking.

\section{Aim}

We performed a systematic review of diagnostic studies assessing the performance of the BAT in patients referred for evaluation of bleeding symptoms.

\section{Methods}

The electronic database PubMed was searched from inception through July 27, 2017. Eligible publications were original studies that assessed and validated the diagnostic accuracy of bleeding questionnaires for identification of adults with mild bleeding disorders. For each study sensitivity, specificity, and diagnostic odds ratio (DOR) were calculated. Quality was assessed using the Quality Assessment of Diagnostic studies-2 tool. To assess influence of specific study characteristics on DOR, univariate metaregression analyses were performed.

\section{Results}

Nine studies were included. Five studies investigating the ISTH BAT or other bleeding questionnaires had a moderate to low DOR. Four studies investigating Vicenza based BATs had a high DOR, with high specificity (>90\%) and sensitivity of $59-85 \%$. Study characteristics such as case-control design, retrospective data collection, and differences in reference standard were associated with optimistic estimates of diagnostic performance. Three out of four studies with a high DOR had these study characteristics. Studies with good methodological quality mainly had a low DOR.

\section{Conclusion}

The main advantage of the BAT is that it offers a complete and structured interview. However, the BAT is of limited diagnostic value to the work-up of patients referred for bleeding evaluation in clinical practice. 


\section{Introduction}

Mild bleeding disorders (MBD) include von Willebrand disease (VWD), platelet function disorders (PFD), and mild clotting factor deficiencies or a combination of defects $^{1}$. Although patients with mild bleeding disorders do not have an increased mortality, they can bleed after surgery and may require blood products or hemostatic agents to control bleeding ${ }^{2}$. Correct diagnosis is essential to apply preventive treatment if necessary. However, compared to severe bleeding disorders, the diagnostic work-up for mild bleeding disorders is much more controversial ${ }^{3}$.

Due to the high prevalence of trivial and minor bleeding symptoms in the general population, the interpretation of bleeding symptoms can be difficult. Minor bleeding symptoms can be easily overlooked and interpreted as normal. On the other hand, extensive analysis of trivial bleeding symptoms may result in unwarranted laboratory testing and false-positive diagnosis ${ }^{4}$. An investigation on the incidence of bleeding symptoms in healthy subjects, showed that bleeding symptoms such as menorrhagia $(47 \%)$, epistaxis $(25 \%)$, easy bruising $(18 \%)$, and prolonged bleeding after dental extraction (18\%) are frequently reported in the healthy population ${ }^{5}$. To distinguish patients with relevant bleeding symptoms indicating an underlying bleeding disorder from healthy subjects with trivial bleeding symptoms, many attempts have been made to standardize and validate tools to report and interpret bleeding history. Throughout the years different bleeding questionnaires, combined with a bleeding score, the so called bleeding assessment tools (BAT), have been developed.

The classical concept of the BAT was realized in $1982^{6}$. This initial model consisted of a questionnaire containing ten questions concerning spontaneous bleeding and bleeding after surgery, dental interventions, and deliveries. A group of investigators from Vicenza, Italy, contributed to further development and validation of the BAT in different populations, in order to offer a quantitative description of the bleeding phenotype, especially in patients with VWD type $1^{3,7,8}$. In 2010, the International Society of Thrombosis and Haemostasis (ISTH) Scientific and Standardization Committee (SSC) Joint Working Group agreed to establish a single BAT, the ISTH BAT, to standardize the reporting and interpretation of bleeding symptoms ${ }^{9}$.

The BAT is increasingly accepted in clinical practice as a valuable method for systematically assessing bleeding symptoms and for improving care for patients with mild bleeding disorders, and it has been increasingly investigated as a diagnostic tool for mild bleeding disorders in patients referred for bleeding evaluation ${ }^{10-13}$. In 2013, a historical review was published about the evolution and value of the BAT ${ }^{14}$. However, a critical appraisal addressing the quality and the results of the validation studies for the BAT is lacking. 


\section{Objective}

To perform a systematic review of diagnostic studies assessing the performance of the BAT in patients referred to a secondary care setting for expert evaluation of bleeding symptoms.

\section{Materials and methods}

\section{Protocol}

First, a review question was formulated: 'What is the diagnostic accuracy of a bleeding assessment tool or bleeding questionnaire for the diagnosis of mild bleeding disorders in adult patients referred to secondary care services for evaluation of bleeding symptoms?'. Then, the methodological quality (risk of bias and applicability concerns) of each study was assessed using the Quality Assessment of Diagnostic studies-2 (QUADAS-2) tool. The PRISMA statement and guidelines of the Cochrane Collaboration were followed ${ }^{15,16}$.

\section{Information sources and search}

A literature search was conducted using the electronic database PubMed. The final search date was July 27,2017 . No language or date restrictions were applied to the electronic search. The terms: 'bleeding disorder OR bleeding tendency' AND 'bleeding questionnaire' were used to identify articles describing the validation of bleeding questionnaires. Case reports and reviews were excluded. All references of eligible studies were checked for other possible relevant titles and this procedure was repeated until no new titles were found. Experts and study authors were contacted and conference proceedings were searched to identify additional studies. Details of search strategy are shown in Appendix 2.A.

\section{Study selection}

Eligible publications were original studies that assessed and validated the diagnostic accuracy of bleeding questionnaires for identification of adults with mild bleeding disorders. Studies had to meet all inclusion criteria: (i) the study had to assess the diagnostic accuracy of questionnaires exclusively addressing bleeding symptoms, (ii) the questionnaire had to be used for identification of patients with mild bleeding disorders (e.g. von Willebrand disease, platelet function disorders or mild coagulation factor deficiencies), (iii) the study population consisted of participants referred to secondary care services, and (iv) absolute numbers of true negative, false negative, true positive, and false positive test results were reported or could be derived. Studies 
which assessed the diagnostic accuracy of questionnaires combining bleeding symptoms with quality of life questions and/or other symptoms like pain, or involving laboratory results were excluded. Studies were excluded if all participants were aged $<18$ years. Publications involving the same patient cohort were included only once.

Two reviewers (FM and EB) independently screened all titles and abstracts, and the full text of potentially relevant articles was obtained. Both reviewers selected articles that met the pre-specified inclusion criteria. Disagreements on final selection of studies were resolved by consensus.

\section{Data extraction}

For each study, two reviewers (FM and SS) completed a standardized data extraction form. The information extracted from the studies included: first author and year of publication, the type of questionnaire used, mode of administration (selfadministered or interview), study design, source of study population, sample size, targeted condition, and choice of reference standard (laboratory results, physician assessment, retrospective review of medical records). Furthermore, the absolute numbers of true negative, false negative, true positive, and false positive test results were derived as well as the cut-off value of the bleeding score that was used for the definition of a positive BAT result. If data were available for multiple cut-off points, the cut-off value with the best results for diagnostic performance was used. If results were presented for different patient groups, the results for the patient group which most closely resembled the patient group in our review question (e.g. referred for evaluation of bleeding symptoms) was selected. The review authors were blinded to each other's data until extraction was complete. Subsequently, the data were compared and discussed, resolving disagreements in data extraction by discussion, involving a third reviewer (PN) when necessary.

\section{Quality assessment}

Two reviewers (FM and SS) independently assessed the methodological quality (risk of bias and applicability concerns) of each study using the Quality Assessment of Diagnostic studies-2 (QUADAS-2) tool, recommended by the Cochrane Collaboration Diagnostic Test Accuracy Working Group. This tool consists of four domains; patient selection, index test, reference standard, and participant flow. For each domain, the risk of bias was assessed using signaling questions (e.g. for patient selection: was a case-control design avoided? $)^{17}$. For the first three domains applicability to our review question was also assessed. To tailor the QUADAS- 2 tool to the review question, one adjustment was made. Following diagnosis of a bleeding disorder, patients are often 
given hemostatic prophylaxis prior to invasive procedures or surgical operations. Treatment of mild bleeding disorders can strongly interfere with the results of the bleeding questionnaire. Therefore the bleeding score had to be obtained before the start of treatment. The signaling question 'was there an appropriate interval between index test and reference standard?' was therefore changed into: 'did the questions of the questionnaire refer to a period before diagnosis or treatment of the bleeding disorder?' (see Appendix 2.B). Disagreements in assessments between the two reviewers were resolved by discussion, involving a third reviewer (PN) if necessary.

\section{Statistical analysis and synthesis of results}

This review focuses on the diagnostic performance of bleeding questionnaires for discrimination between presence and absence of a mild bleeding disorder. For each study, sensitivity and specificity as well as diagnostic odds ratio (DOR) with $95 \%$ confidence intervals were calculated. The DOR combines sensitivity and specificity into a single indicator of test performance and is not affected by differences in cut-off points (for a positive test result) between studies. A DOR of 1 means that a test does not discriminate between patients with and without disease. Higher values indicate better test performance. In case of sensitivity or specificity estimates of $100 \%$, the $2 \times 2$ tables including true positive, false positive, false negative, and true negative test results contain zero values. Therefore 0.5 was added to all counts in the $2 \times 2$ table to calculate an approximation of the $D O R^{18}$. Data are presented in forest plots and a summary receiver operating characteristic (SROC) curve. To assess whether or not the presence (vs absence) of specific study characteristics influenced the variation in DOR from individual studies, univariate meta-regression analyses were performed and relative diagnostic odds ratios (RDOR) with 95\% confidence intervals were calculated $^{19}$. The RDOR represents the pooled DOR for studies with a specific characteristic divided by the pooled DOR of studies without that specific characteristic. Furthermore, the $\mathrm{R}^{2}$ index, representing the amount of variation in DOR explained by a specific study characteristic was calculated. Analyses were performed with Review Manager 5 and R (version 3.3.2). R packages Mada and Metafor were used. $P$-values $\leq 0.05$ were considered to indicate statistical significance.

\section{Results}

\section{Study selection}

The literature search resulted in 1691 citations and three records were identified through other sources (reference list, contacting an expert, and one conference 
proceeding). For 37 potentially relevant studies, the full-text articles were derived. A total of nine studies met the inclusion criteria ${ }^{7,8,10-13,20-22}$. Reasons for exclusion were: the study appeared not to be a validation study; appeared to be a post script or letter to the editor; to validate a combined questionnaire; to investigate a primary care patient population, or a population of only children (Figure 2.1). Full references of included studies are available in Appendix 2.C.

\section{Figure 2.1 PRISMA flow diagram.}

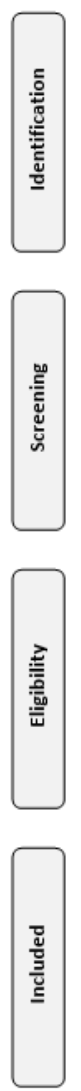

Records identified through database searching $(n=1691)$

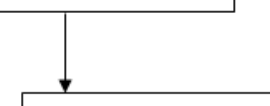

Records after d $(n=1688)$
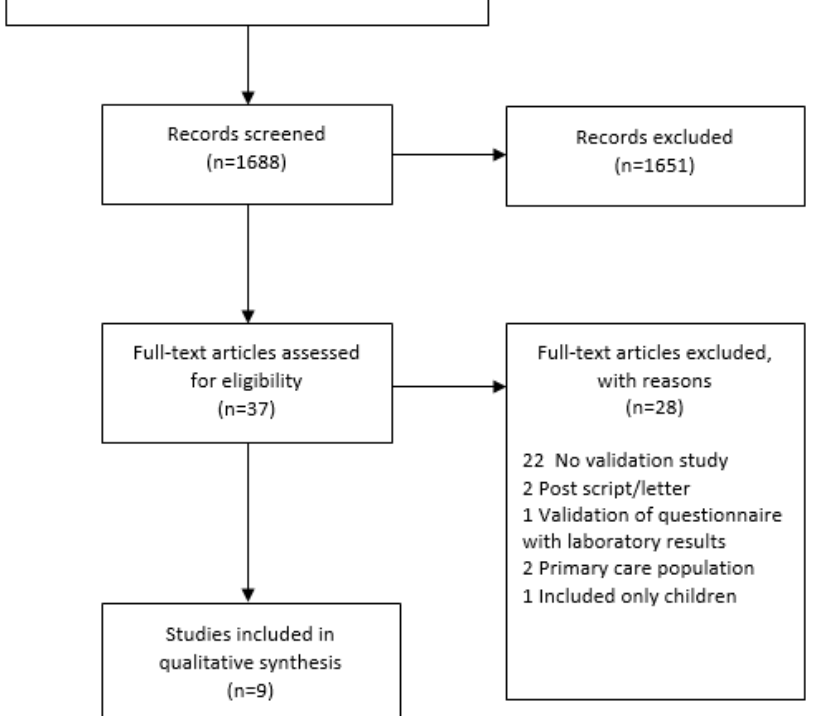

Article search and selection, other sources included reference lists, expert contact, and conference proceedings. $n$, number.

\section{Study characteristics}

The study characteristics of the nine included studies are summarized in Table 2.1. Five studies investigated Vicenza based bleeding assessment tools (the Vicenza BAT, the MCMDM-1 VWD BAT or the condensed MCMDM-1 VWD BAT). Two studies 
applied the ISTH BAT. The two other studies used a bleeding questionnaire specifically designed for the study or for a specific bleeding problem (menorrhagia). The targeted condition, mild bleeding disorders, was limited to VWD in four studies, one study focused only on platelet function disorders as MBD and the four remaining studies included a combination of different mild bleeding disorders (VWD, PFD, and mild coagulation factor deficiencies).

\section{Diagnostic test accuracy of bleeding questionnaires}

The sensitivity and specificity with 95\% confidence intervals for each study, with specification of investigated questionnaire and bleeding disorder are presented in Figure 2.2. Sensitivity in the studies ranged from $33 \%$ to $89 \%$, specificity ranged from $16 \%$ to $100 \%$. Figure 2.3 presents a summary ROC curve based on the pairs of sensitivity and specificity of the nine individual studies. This figure shows large heterogeneity in study results as the observed pairs of sensitivity and specificity from the individual studies scatter around the summary ROC curve. The DORs with 95\% confidence intervals are presented in Figure 2.4. The DORs from individual studies range from 0.86 to 151 . Four studies reported high diagnostic performance with DORs ranging from 36 to 151, two studies reported moderate performance (DOR 6.7 and 3.5 , respectively), and three studies reported DORs around 1 indicating lack of discriminating power of the questionnaire between patients with and without MBD. The four studies reporting high DOR used Vicenza based questionnaires and predominantly focused on the diagnosis of VWD. All of these studies reported high specificity with a wide range in sensitivity (59 to $85 \%$ ).

Figure 2.2 Forest plot of sensitivity and specificity for each study.

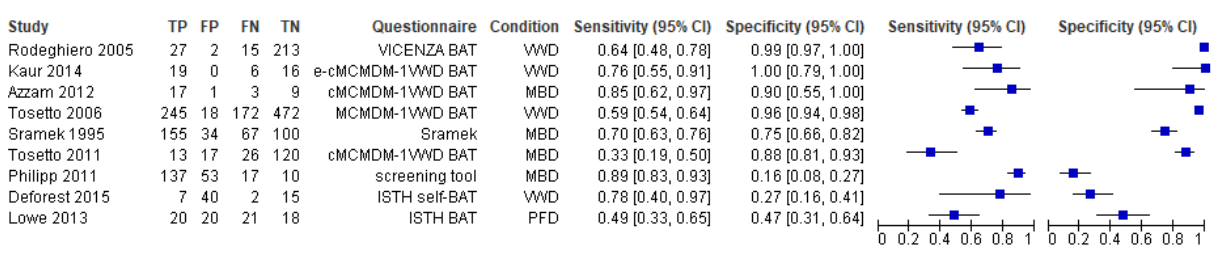

Sensitivity and specificity with 95\% confidence interval for each study with specification of investigated questionnaire and bleeding disorder. BAT, bleeding assessment tool; $\mathrm{C}$, condensed; $\mathrm{Cl}$, confidence interval; FN, false negative; FP, false positive; ISTH, International Society of Thrombosis and Haemostasis; TN, true negative; TP, true positive; MBD, mild bleeding disorders; MCMDM-1 VWD, Molecular and Clinical Markers for the Diagnosis and Management of Type 1 von Willebrand disease; PFD, platelet function disorder; VWD, von Willebrand disease. 


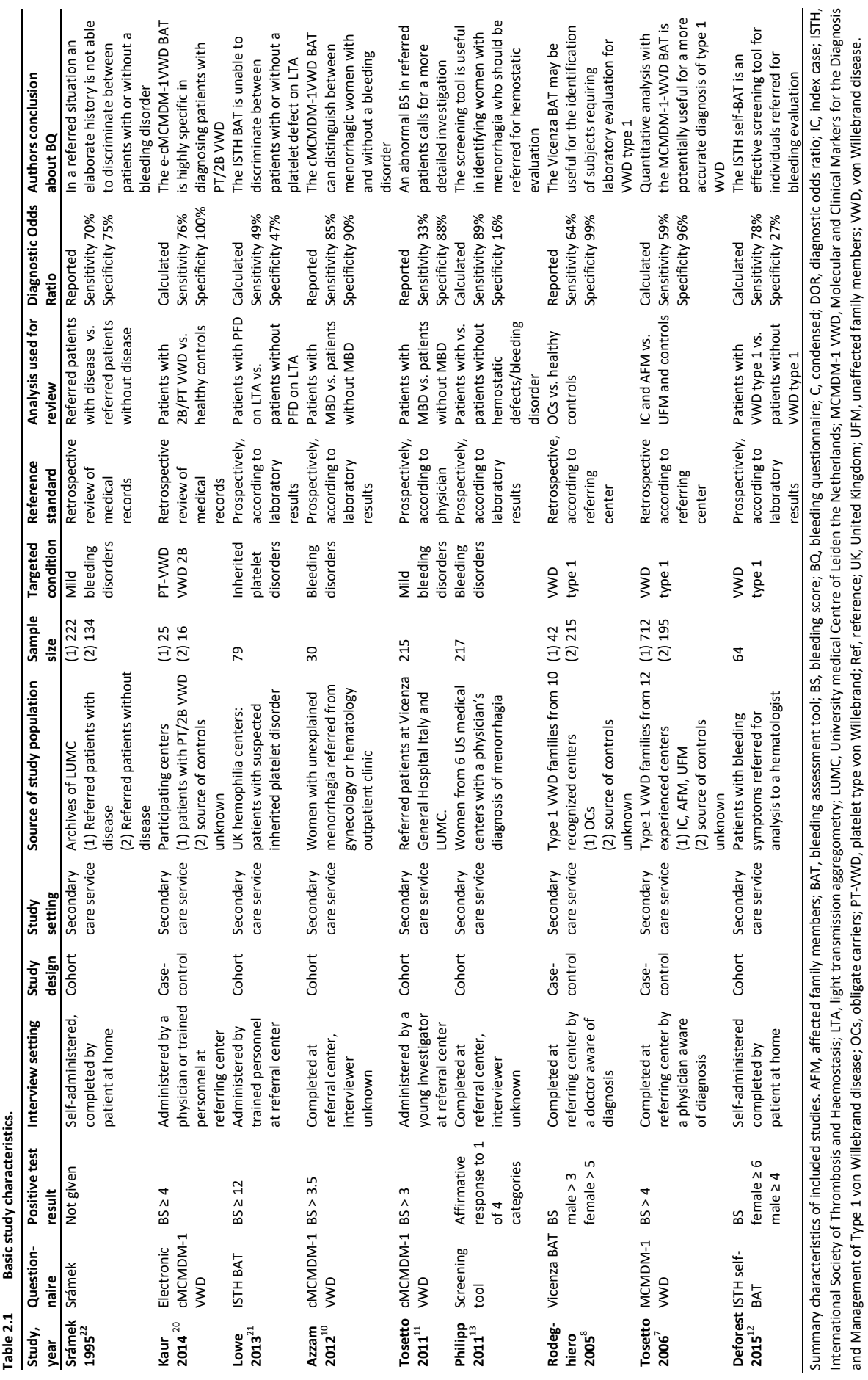




\section{Methodological quality of the studies}

The methodological quality of the studies was assessed with the QUADAS-2. A summary of the risk of bias and applicability concerns of each study is presented in Figure 2.5. Risk of bias was considered high when studies used a case-control design $(n=3)$ or retrospective data collection $(n=4)$, when blinding to final diagnosis was inadequate $(n=5)$ or when the diagnostic criteria for the targeted conditions (reference standard) were not well described $(n=4)$. In four studies the diagnosis was not verified in the same way in all patients (patient flow: differences in reference standard). High loss to follow-up of $23 \%$ without clear reason was observed in one study.

Figure 2.3 SROC curve for all studies with summary estimate and confidence region.

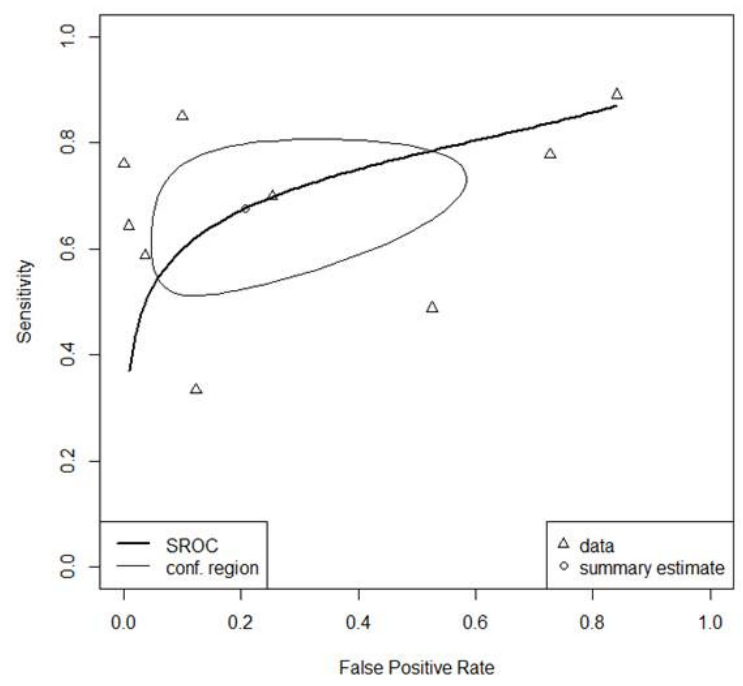

The SROC curve (bivariate model) based on the pairs of sensitivity and specificity of the nine individual studies with summary estimate and confidence region. Due to large heterogeneity in study results area under the SROC curve was not calculated. Conf, confidence; SROC, summary receiver operating characteristic. 
Figure 2.4 Diagnostic Odds Ratio (DOR) with 95\% confidence interval.

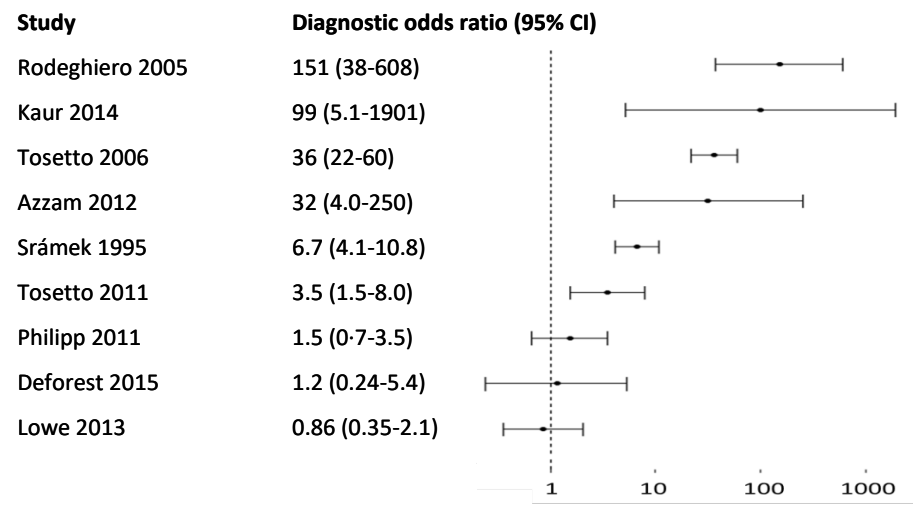

For each study the diagnostic odds ratio (DOR) was calculated with $95 \%$ confidence interval. $\mathrm{Cl}$, confidence interval; DOR, diagnostic odds ratio.

Figure 2.5 Risk of bias and applicability concerns (QUADAS-2).

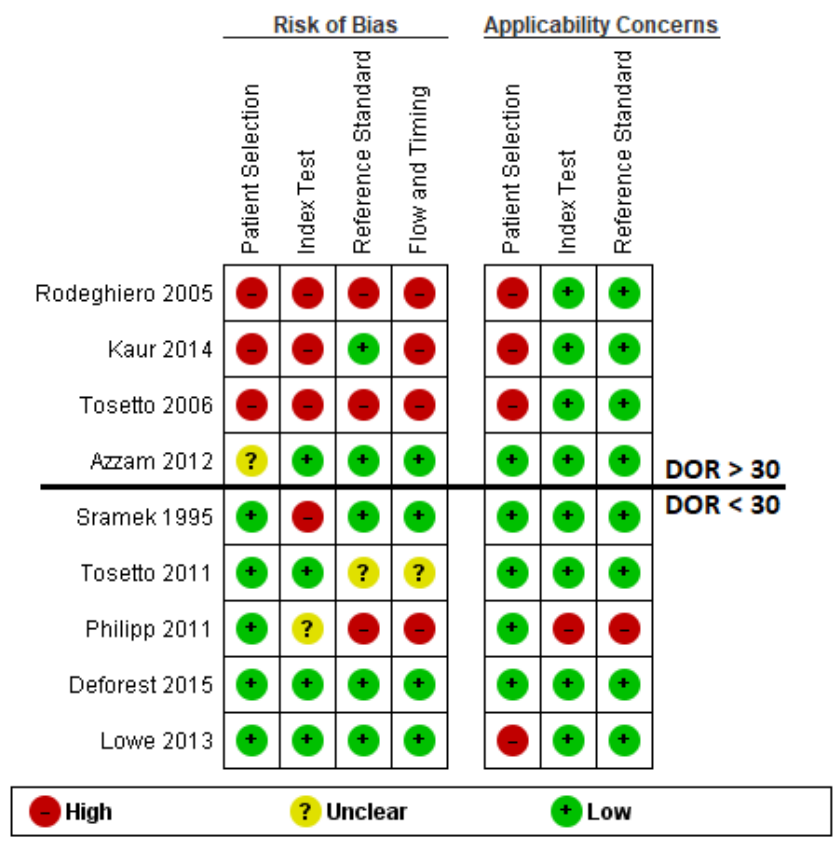

Summary of the results for risks of bias and applicability concerns according to the Quality Assessment of Diagnostic studies-2 (QUADAS-2). Studies above the bold line had a DOR of more than 30, studies below the bold line had a DOR of less than 30. DOR, diagnostic odds ratio; QUADAS-2, Quality Assessment of Diagnostic studies-2. 


\section{Effect of study characteristic on DOR}

Studies were categorized into subgroups according to the presence or absence of specific study characteristics. Table 2.2 shows the distribution of study characteristics across the studies. For each study characteristic the relative diagnostic odds ratios (RDOR) with $95 \%$ confidence intervals and $\mathrm{R}^{2}$ is shown in Figure 2.6. Studies with a case-control design, retrospective data collection, and differences in reference standard tended to report higher DORs. These study characteristics, which explain part of the heterogeneity between study results, were present in three of four studies with high DOR and in two of five studies with low DOR. The other study characteristics had no significant effect on DOR and offered no significant explanation for the variation in DOR across the studies.

Table 2.2 Distribution of study characteristics across the studies.

\begin{tabular}{llc}
\hline Study characteristic & & Number of studies \\
\hline Patient selection & Cohort & 6 \\
& Case-control & 3 \\
\hline Data collection & Prospective & 5 \\
& Retrospective & 4 \\
\hline Index test & Blinding of final diagnosis & 4 \\
& No or unclear blinding of final diagnosis & 5 \\
\hline Reference test & Clear definition of disease & 5 \\
& No definition of disease & 4 \\
\hline Patient flow & No differences in reference standard & 5 \\
& Differences in reference standard & 4 \\
\cline { 2 - 3 } & No or clear loss to follow-up & 8 \\
& High or unclear loss to follow-up & 1 \\
\hline Target disease & von Willebrand disease & 4 \\
& Other mild bleeding disorders & 5 \\
\cline { 2 - 3 } & Platelet function disorder & 1 \\
& Other mild bleeding disorders & 8 \\
\hline
\end{tabular}

Distribution of study characteristics across the studies.

\section{Predictive value of bleeding questionnaires}

The positive and negative predictive value of a test strongly depends on the prevalence of the disease. For illustration purposes, Figure 2.7 shows the post-test probability of having a MBD in patients with a positive (A) or negative (B) BAT according to the pre-test probability (disease prevalence). Predictive values were calculated based on the sensitivity and specificity for all studies. As anticipated for the studies with low DOR, the results show that the likelihood of a mild bleeding disorder is not influenced by a positive or negative BAT result. The results of studies with high DOR show that the likelihood of a mild bleeding disorder, especially VWD, becomes 
higher when the patient has a positive test result. Furthermore, although the likelihood of a mild bleeding disorder becomes lower when the patient has a negative test result, MBD cannot be excluded definitely and especially at higher pre-test probabilities, the probability that the patient has a mild bleeding disorder still remains high.

Figure 2.6 Relative diagnostic odds ratios for study characteristics.

Study Characteristic ( $\mathbf{n}$ )
Case control (3)
Retrospective data (4)
Differences in ref. test (4)
Target disease VWD (4)
No blinding final diagnosis (5)
Target disease PFD (1)
No definition disease (4)

Relative Diagnostic Odds ratio $(95 \% \mathrm{Cl})$

25.2 (5.1 - 126)

$14.8(2.3-91.2)$

$10.7(1.3-90.1)$

$7.89(0.8-76.4)$

$6.15(0.5-69.9)$

$0.07(0.0-2.9)$

$2.06(0.1-30.8)$

$\begin{array}{ll}\boldsymbol{P} \text { value } & \mathbf{R}^{2} \text { (\%) } \\ <0.01 & 75 \\ <0.01 & 56 \\ 0.03 & 37 \\ 0.07 & 29 \\ 0.14 & 15 \\ 0.87 & 12 \\ 0.60 & 0\end{array}$

$\begin{array}{lllll}0.01 & 0.1 & 1 & 10 & 100\end{array}$

A univariate meta-regression was performed to calculate the relative diagnostic odds ratio (RDOR). The RDOR represents the pooled DOR for studies with a specific characteristic divided by the pooled DOR of studies without that specific characteristic. The $R^{2}$ index represents the amount of variation in DOR explained by specific study characteristic. $n$ represents the number of studies with that specific study characteristic. $P$ value below 0.05 is significant. $\mathrm{Cl}$, confidence interval; $\mathrm{n}$, number; RDOR, relative diagnostic odds ratio; ref, reference. 
Figure 2.7 Post-test probability of having a mild bleeding disorder according to test results and pretest probability.
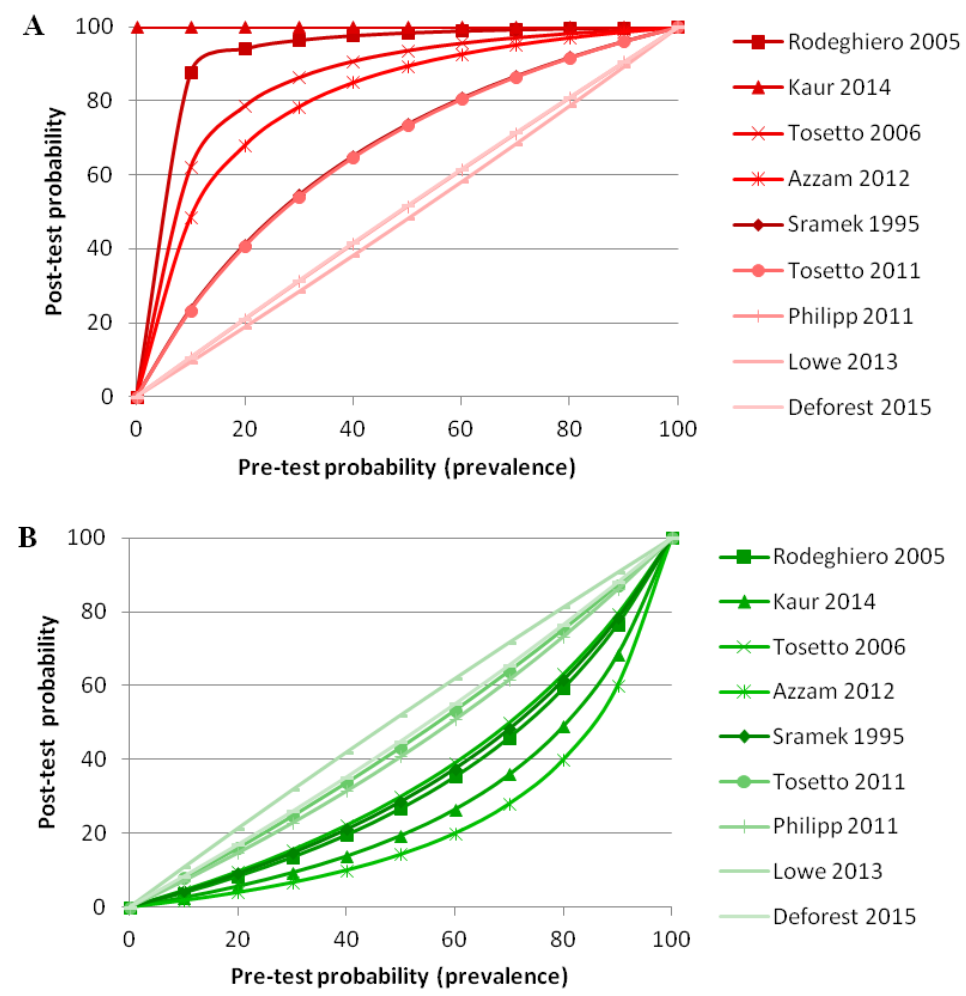

Post-test probability of having a mild bleeding disorder according to test results and pre-test probability (prevalence). (A) post-test probability of MBD in patients with a positive test result. (B) post-test probability of MBD in patients with a negative test result.

\section{Discussion}

This systematic review assessed the performance of the BAT in patients referred to secondary care services for expert evaluation of bleeding symptoms. We analyzed nine studies. There was a wide range in sensitivity and specificity across the different studies, and there was a large heterogeneity in study results.

Five studies investigating the ISTH BAT or other bleeding questionnaires had a moderate to low DOR indicating that the BAT is not very helpful in discriminating patients with and without mild bleeding disorders. Four studies investigating Vicenza based BATs in patients with predominantly VWD, reported a high DOR, indicating good discriminative ability for this disease. These studies had a high specificity 
indicating that a positive BAT increases the patient's likelihood of having a MBD, especially VWD, requiring a more detailed investigation of the patient ${ }^{11,23}$. Sensitivity of these studies ranged from 59 to $85 \%$ indicating that the BAT is not able to definitely exclude a MBD and there remains a substantial chance that the patient has a mild bleeding disorder despite a negative BAT. The low sensitivity of the BAT may be explained by the fact that many patients with MBD are pauci-symptomatic and, in the case of younger patients, there is less exposure to challenging events that predispose to bleeding.

Many studies investigating the BAT claim that a MBD can be excluded with a negative BAT and that the greatest clinical utility lies in the high negative predictive value of the BAT and its ability to exclude subjects in which further testing may be unnecessary $3,8,11,12$. Our findings do not support this claim. The high NPV in these studies is misleading, due to the low disease prevalence in the studied population, varying from 1 to $14 \%$. Since most patients do not have a MBD, there are many true negative test results, but the lower sensitivity in these studies (33-78\%) indicates that the few patients with a MBD still could be missed by the BAT. The prevalence of MBD in patients referred to secondary care services for bleeding evaluation can range up to $44 \%^{11,24}$. Therefore, the NPV calculated in the referred population with higher disease prevalence is expected to be much lower.

A positive BAT increases the likelihood of having a MBD. Yet, since there are no distinctive symptoms in patients suffering from different mild bleeding disorders and there is no difference in bleeding scores between patients with VWD, PFD, and clotting factor deficiencies, the BAT alone cannot diagnose a specific disorder ${ }^{24}$. A positive BAT might justify extensive laboratory testing ${ }^{8,11}$ and Tosetto et al. ${ }^{25}$ stated that the BAT should be integrated in a full diagnostic algorithm including laboratory and family data.

The BAT offers a structured and standardized diagnostic approach, especially for unexperienced physicians and when standardization of clinical assessment is mandatory (e.g. in clinical studies). However, an important disadvantage of performing the BAT and computing a bleeding score in clinical practice is that it is time consuming (taking up to 45 minutes).

One might dispute the additional value of an elaborate BAT with a complex bleeding score to the medical history taking of an experienced physician. A study by Rodeghiero et al. $^{8}$ showed no superiority between a simple criterion (having more than two bleeding symptoms) and the more elaborate bleeding score to define a bleeding history distinguishing VWD patients from normal subjects. In a study by Tosetto et al. ${ }^{11}$ the bleeding history was assessed by a young investigator administering the BAT and by a senior physician who independently evaluated the 
patient and made the final diagnoses. They found that of the 215 patients enrolled in the study only eleven with a positive BAT were classified by the physician as having no bleeding disorder. These patients had no laboratory abnormalities and were previously identified as healthy subjects with mucocutaneous bleeding of uncertain clinical relevance ${ }^{26}$. Another study compared the bleeding score derived from a standardized questionnaire with the insight of the physician who classified the bleeding severity at the end of the interview into one of five clinical bleeding categories. A good correlation was found between the bleeding score and the estimation of the physician. The authors concluded that performing and analyzing a time consuming bleeding score was not better than an interview with a preestablished questionnaire followed by the classification of the bleeding severity by the physician ${ }^{24,26}$.

Furthermore, the additional value of the increased likelihood of having a MBD when the BAT is positive, may also be less pronounced, because in hematological practices, the process of referral (often because of bleeding symptoms) means that the patients is already likely to have a bleeding problem. This consideration is in line with a previous review where it was stated that the value of a BAT in determining pretest likelihood for mild bleeding disorders seems limited ${ }^{27}$.

The assessment of methodological quality revealed that risk of bias was present in the majority of studies. Studies with a case-control study design, retrospective data collection, or differences in reference standard tended to report more optimistic estimates of diagnostic performance. These study characteristics were present in three of four studies with a high DOR investigating Vicenza based BATs and diagnostic performance may have been overestimated. Of the four studies with good methodological quality, three had a low DOR.

This study has some limitations. An important problem is lack of a generally accepted gold standard for the diagnosis of MBD. Even more, most of the diagnostic criteria used in the included studies are largely based on laboratory findings, and there is an acknowledged lack of standardization in the laboratory approach of testing for mild bleeding disorders. Tests for specific disorders (e.g. LTA for platelet function defects) are known to have inter-user variability ${ }^{24}$. Lack of gold standard hampers proper evaluation of diagnostic tests. The development of new diagnostic tests, especially DNA analyses, might lead to a better gold standard and improve future research. Furthermore, several studies evaluated only one specific disorder. The sensitivity and specificity in these studies may only reflect the disorder tested for and not necessarily all mild bleeding disorders. Finally, only a small number of studies met the inclusion criteria and could be included in this review. The effect of methodological shortcomings were evaluated by univariate meta-regression analyses, 
but multivariable analyses that can adjust for residual confounding by other study characteristics was not feasible.

\section{Conclusion}

Four studies investigating Vicenza based BATs in patients with predominantly VWD, reported a high DOR, indicating good discriminative ability for this disease. These studies had a high specificity indicating that a positive BAT increases the patient's likelihood of having a MBD, calling for a more detailed investigation of the patient. The sensitivity of the four studies investigating Vicenza based bleeding assessment tools in patients with predominantly VWD ranged from 59 to $85 \%$ indicating that a BAT is not able to definitely exclude a MBD, and there remains a substantial chance that the patient has a mild bleeding disorder despite a negative BAT. Five of nine studies investigating the ISTH BAT or other bleeding questionnaires had a low to moderate DOR, indicating that the BAT is not very helpful in discriminating patients with and without mild bleeding disorders.

We found a trend towards lower DORs for studies with good methodological quality. Methodological shortcomings in three of four studies with a high DOR investigating Vicenza based BATs may have resulted in overestimation of diagnostic performance. We believe that the main advantage of the BAT is that it offers a complete and structured interview, especially in settings were standardization of clinical assessment is mandatory. However, the BAT is of limited additional diagnostic value to the work-up of patients referred for bleeding evaluation in clinical practice. 


\section{References}

1 Mezzano D, Quiroga T. Diagnostic challenges of inherited mild bleeding disorders: a bait for poorly explored clinical and basic research. J Thromb Haemost 2019; 17: 257-70.

2 Orsini S, Noris $\mathrm{P}$, Bury $\mathrm{L}$, et al. Bleeding risk of surgery and its prevention in patients with inherited platelet disorders. Haematologica 2017; 102: 1192-203.

3 Bowman M, Mundell G, Grabell J, Hopman WM, Rapson D, Lillicrap D, James P. Generation and validation of the Condensed MCMDM-1VWD Bleeding Questionnaire for von Willebrand disease. Journal of Thrombosis and Haemostasis 2008; 6: 2062-6.

4 Tosetto A, Castaman G, Rodeghiero F. Bleeders, bleeding rates, and bleeding score. J Thromb Haemost 2013; 11 Suppl 1: 142-50.

5 Mauer AC, Khazanov NA, Levenkova N, et al. Impact of sex, age, race, ethnicity and aspirin use on bleeding symptoms in healthy adults. Journal of Thrombosis and Haemostasis 2011; 9: 100-8.

6 Wahlberg T. Carriers and noncarriers of haemophilia A. Evaluation of bleeding symptoms registered by a self-administered questionnaire with binary (no/yes) questions. Thromb Res 1982; 25: 415-22.

7 Tosetto A, Rodeghiero F, Castaman G, et al. A quantitative analysis of bleeding symptoms in type 1 von Willebrand disease: results from a multicenter European study (MCMDM-1 VWD). Journal of Thrombosis and Haemostasis 2006; 4: 766-73.

8 Rodeghiero F, Castaman G, Tosetto A, et al. The discriminant power of bleeding history for the diagnosis of type 1 von Willebrand disease: an international, multicenter study. J Thromb Haemost 2005; 3: 2619-26.

9 Rodeghiero F, Tosetto A, Abshire T, et al. ISTH/SSC bleeding assessment tool: a standardized questionnaire and a proposal for a new bleeding score for inherited bleeding disorders. J Thromb Haemost 2010; 8: 2063-5.

10 Azzam HA, Goneim HR, El-Saddik AM, Azmy E, Hassan M, El-Sharawy S. The condensed MCMDM-1 VWD bleeding questionnaire as a predictor of bleeding disorders in women with unexplained menorrhagia. Blood Coagul Fibrinolysis 2012; 23: 311-5.

11 Tosetto A, Castaman G, Plug I, Rodeghiero F, Eikenboom J. Prospective evaluation of the clinical utility of quantitative bleeding severity assessment in patients referred for hemostatic evaluation. J Thromb Haemost 2011; 9: 1143-8.

12 Deforest M, Grabell O, Albert S, Young J, Tuttle A, Hopman WM, James PD. Generation and optimization of the self-administered bleeding assessment tool and its validation as a screening test for von Willebrand disease. Haemophilia 2015; 21: E384-E8.

13 Philipp CS, Faiz A, Heit JA, et al. Evaluation of a screening tool for bleeding disorders in a US multisite cohort of women with menorrhagia. Am J Obstet Gynecol 2011; 204: 209 e1-7.

14 Rydz N, James PD. The evolution and value of bleeding assessment tools. J Thromb Haemost 2012; 10: 2223-9.

15 Moher D, Liberati A, Tetzlaff J, Altman DG, Group P. Preferred reporting items for systematic reviews and meta-analyses: the PRISMA statement. Int J Surg 2010; 8: 336-41.

16 Deeks JJ WS, Davenport C. . Chapter 4: Guide to the contents of a Cochrane Diagnostic Test Accuracy Protocol. Cochrane Handbook for Systematic Reviews of Diagnostic Test Accuracy Version 1.0.0. . 2013.

17 Rutjes AW, Reitsma JB, Vandenbroucke JP, Glas AS, Bossuyt PM. Case-control and two-gate designs in diagnostic accuracy studies. Clin Chem 2005; 51: 1335-41.

18 Glas AS, Lijmer JG, Prins MH, Bonsel GJ, Bossuyt PM. The diagnostic odds ratio: a single indicator of test performance. J Clin Epidemiol 2003; 56: 1129-35.

19 Lijmer JG, Mol BW, Heisterkamp S, Bonsel GJ, Prins MH, van der Meulen JH, Bossuyt PM. Empirical evidence of design-related bias in studies of diagnostic tests. JAMA 1999; 282: 1061-6.

20 Kaur H, Ozelo M, Scovil S, James PD, Othman M. Systematic analysis of bleeding phenotype in PTVWD compared to type $2 \mathrm{~B}$ VWD using an electronic bleeding questionnaire. Clin Appl Thromb Hemost 2014; 20: 765-71. 
21 Lowe GC, Lordkipanidze M, Watson SP, group UGs. Utility of the ISTH bleeding assessment tool in predicting platelet defects in participants with suspected inherited platelet function disorders. J Thromb Haemost 2013; 11: 1663-8.

22 Sramek A, Eikenboom JCJ, Briet E, Vandenbrouche JP, Rosendaal FR. Usefulness of Patient Interview in Bleeding Disorders. Archives of Internal Medicine 1995; 155: 1409-15.

23 Rodeghiero F, Kadir RA, Tosetto A, James PD. Relevance of quantitative assessment of bleeding in haemorrhagic disorders. Haemophilia 2008; 14 Suppl 3: 68-75.

24 Quiroga T, Mezzano D. Is my patient a bleeder? A diagnostic framework for mild bleeding disorders. Hematology Am Soc Hematol Educ Program 2012; 2012: 466-74.

25 Tosetto A, Castaman G, Rodeghiero F. Evidence-based diagnosis of type 1 von Willebrand disease: a Bayes theorem approach. Blood 2008; 111: 3998-4003.

26 Quiroga T, Goycoolea M, Panes O, et al. High prevalence of bleeders of unknown cause among patients with inherited mucocutaneous bleeding. A prospective study of 280 patients and 299 controls. Haematologica 2007; 92: 357-65.

27 Hayward CP. Diagnosis and management of mild bleeding disorders. Hematology Am Soc Hematol Educ Program 2005: 423-8. 


\section{Appendix 2.A Search strategy}

Relevant studies published from inception to July 27, 2017 (date last searched), were identified through an electronic search using the Pubmed database. Electronic search was supplemented by scanning reference lists of articles identified for all relevant studies (including review articles). Experts and study authors were contacted and conference proceedings were searched to identify additional studies. The computer-based search combined search terms related to bleeding disorders and bleeding questionnaire.

01. "bleeding disorder" (97756)

02. "bleeding tendency" (96942)

03. "bleeding questionnaire" (17351)

04. ((bleeding disorder) OR bleeding tendency) AND bleeding questionnaire (1426)

Query translations 04:

(("blood coagulation disorders"[MeSH Terms] OR ("blood"[All Fields] AND "coagulation"[All Fields] AND

"disorders"[All Fields]) OR "blood coagulation disorders"[All Fields] OR ("bleeding"[All Fields] AND

"disorder"[All Fields]) OR "bleeding disorder"[All Fields]) AND ("blood coagulation disorders"[MeSH Terms] OR ("blood"[All Fields] AND "coagulation"[All Fields] AND "disorders"[All Fields]) OR "blood coagulation disorders"[All Fields] OR ("bleeding"[All Fields] AND "tendency"[All Fields]) OR "bleeding tendency"[All Fields])) AND (("hemorrhage"[MeSH Terms] OR "hemorrhage"[All Fields] OR "bleeding"[All Fields]) AND ("surveys and questionnaires"[MeSH Terms] OR ("surveys"[All Fields] AND "questionnaires"[All Fields]) OR "surveys and questionnaires"[All Fields] OR "questionnaire"[All Fields])) 


\section{Appendix 2.B Signaling questions for risk of bias and applicability concerns assessment}

\begin{tabular}{|c|c|}
\hline $\begin{array}{l}\text { Risk of bias } \\
\text { Domain }\end{array}$ & Signaling questions \\
\hline \multirow[t]{3}{*}{ Patient selection } & Was a consecutive or random sample of patients enrolled? \\
\hline & Was a case-control design avoided? \\
\hline & Did the study investigators avoid inappropriate exclusions? \\
\hline \multirow[t]{2}{*}{ Index test } & $\begin{array}{l}\text { Were the index test results interpreted without knowledge of the results of the } \\
\text { reference standard? }\end{array}$ \\
\hline & If a threshold was used, was it pre-specified? \\
\hline \multirow[t]{2}{*}{ Reference standard } & Is the reference standard likely to correctly classify the target condition? \\
\hline & $\begin{array}{l}\text { Were the reference standard results interpreted without knowledge of the results } \\
\text { of the index test? }\end{array}$ \\
\hline \multirow[t]{4}{*}{ Study flow } & $\begin{array}{l}\text { Did the questions only regard the time before diagnosis or treatment of the } \\
\text { bleeding disorder? }\end{array}$ \\
\hline & Did all patients receive a reference standard? \\
\hline & Did patients receive the same reference standard? \\
\hline & Were all patients included in the analysis? \\
\hline \multicolumn{2}{|l|}{ Applicability concerns } \\
\hline Domain & Signaling questions \\
\hline Patient & Are there concerns that the included patients do not match the review question? \\
\hline Index test & $\begin{array}{l}\text { Are there concerns that the index test, its conduct, or interpretation differ from } \\
\text { the review question? }\end{array}$ \\
\hline Reference standard & $\begin{array}{l}\text { Are there concerns that the target condition as defined by the reference standard } \\
\text { does not match the review question? }\end{array}$ \\
\hline
\end{tabular}




\section{Appendix 2.C References of included studies}

Azzam HA, Goneim HR, El-Saddik AM, Azmy E, Hassan M, El-Sharawy S. The condensed MCMDM-1 VWD bleeding questionnaire as a predictor of bleeding disorders in women with unexplained menorrhagia. Blood Coagul Fibrinolysis 2012; 23: 311-5.

Deforest M, Grabell J, Albert S, Young J, Tuttle A, Hopman WM, James PD. Generation and optimization of the self-administered bleeding assessment tool and its validation as a screening test for von Willebrand disease. Haemophilia 2015; 21: e384-8.

Kaur H, Ozelo M, Scovil S, James PD, Othman M. Systematic analysis of bleeding phenotype in PT-VWD compared to type 2B VWD using an electronic bleeding questionnaire. Clin Appl Thromb Hemost 2014; 20 : 765-71.

Lowe GC, Lordkipanidze M, Watson SP, group UGs. Utility of the ISTH bleeding assessment tool in predicting platelet defects in participants with suspected inherited platelet function disorders. J Thromb Haemost 2013; 11: 1663-8.

Philipp CS, Faiz A, Heit JA, et al. Evaluation of a screening tool for bleeding disorders in a US multisite cohort of women with menorrhagia. Am J Obstet Gynecol 2011; 204: 209 e1-7.

Rodeghiero F, Castaman G, Tosetto A, et al. The discriminant power of bleeding history for the diagnosis of type 1 von Willebrand disease: an international, multicenter study. J Thromb Haemost 2005; 3: 2619-26.

Srámek A, Eikenboom JC, Briet E, Vandenbroucke JP, Rosendaal FR. Usefulness of patient interview in bleeding disorders. Arch Intern Med 1995; 155: 1409-15.

Tosetto A, Castaman G, Plug I, Rodeghiero F, Eikenboom J. Prospective evaluation of the clinical utility of quantitative bleeding severity assessment in patients referred for hemostatic evaluation. J Thromb Haemost 2011; 9: 1143-8.

Tosetto A, Rodeghiero F, Castaman G, et al. A quantitative analysis of bleeding symptoms in type 1 von Willebrand disease: results from a multicenter European study (MCMDM-1 VWD). J Thromb Haemost 2006; 4: 766-73. 



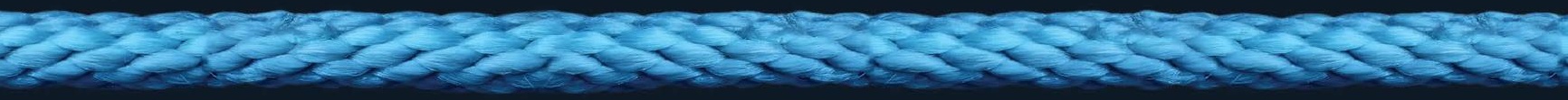




\section{CHAPTER 3}

\section{Screening for platelet function disorders with Multiplate and platelet function analyzer}

Floor C.J.I. Moenen, Minka J.A. Vries, Patricia J. Nelemans, Katrien J.M. van Rooy, Jeannique R.R.A. Vranken, Paul W.M. Verhezen, Rick J. H. Wetzels, Hugo ten Cate, Harry C. Schouten, Erik A.M. Beckers, Yvonne M.C Henskens 


\section{Abstract}

\section{Introduction}

Light transmission aggregation (LTA) is the gold standard for the diagnosis of platelet function disorders (PFD), but is time consuming and limited to specialized laboratories. Whole blood impedance aggregometry (Multiplate) and platelet function analyzer (PFA) may be used as rapid screening tools to exclude PFD.

\section{Aim}

The aim of this study is to assess the diagnostic performance of Multiplate and PFA for platelet function disorders, as detected by LTA.

\section{Methods}

Data from preoperative patients, patients referred to the hematologist for bleeding evaluation and patients with a diagnosed bleeding disorder were used. PFD were defined as $\geq 2$ abnormal LTA curves. Diagnostic performance of Multiplate (ASPItest, TRAPtest, COLtest, ADPtest) and PFA (C-EPI, C-ADP) for detecting PFD was expressed as sensitivity and specificity. The ability of Multiplate agonists and PFA kits to detect corresponding LTA curve abnormalities was expressed as area under the receiver operating characteristic curve (AUC).

\section{Results}

Prevalence of PFD was 16/335 (5\%) in preoperative patients, 10/54 (19\%) in referred patients and $3 / 25(12 \%)$ in patients with a diagnosed bleeding disorder. In preoperative and referred patients, the sensitivity of Multiplate and PFA for detecting mild PFD varied between $0 \%$ and $40 \%$ and AUCs for detecting corresponding LTA curve abnormalities were close to 0.50 . In patients with a diagnosed bleeding disorder, both assays could detect Glanzmann Thrombasthenia (GT) with sensitivity of $100 \%$ and AUCs of $0.70-1.00$.

\section{Conclusion}

Multiplate and PFA cannot discriminate between preoperative and referred patients with and without mild platelet function disorders, meaning that they cannot be used as screening tests to rule out mild platelet function disorders in these populations. Both Multiplate and PFA can detect GT in previously diagnosed patients. 


\section{Introduction}

Platelets are critically involved in normal hemostasis and pathological bleeding ${ }^{1,2}$, and platelet function testing is commonly performed in the diagnostic work-up of patients with a bleeding tendency, or patients at risk of interventional bleeding. Many clinical laboratories evaluate platelet function by light transmission aggregometry (LTA), which is considered the gold standard for the assessment of platelet function ${ }^{3,4}$. By using a panel of agonists that activate platelets via different receptors, LTA can detect many well characterized platelet function disorders (PFD). LTA primarily tests for platelet aggregation defects (e.g. Glanzmann thrombasthenia), but curve interpretation also gives information about platelet adhesion and secretion (second wave) and certain findings are characteristic for platelet adhesion defects (BernardSoulier syndrome) or highly suggest platelet secretion defects.

LTA is however, a time consuming technique and affected by different preanalytical conditions (e.g. lipemic plasma, low platelet count) as well as by different procedural conditions (e.g. platelet rich plasma (PRP) preparation, different agonist concentrations $)^{5}$. A high degree of experience and expertise is necessary to perform and interpret this test, limiting its use to specialized laboratories ${ }^{1,2,6}$. To overcome these limitations, other less laborious whole blood platelet function tests and primary hemostatic function tests have been developed and used, such as impedance aggregometry (Multiplate) and platelet function analyzer (PFA). The procedures of these assays are easier and faster, they have disposable cartridges or cups, are less influenced by (pre-)analytical procedures ${ }^{7-9}$ and do not require a specialized laboratory ${ }^{1}$. The use of anticoagulated whole blood eliminates the need for timeconsuming centrifugation steps and less blood is needed. Multiplate and PFA do not provide the same extent of information as LTA (characteristics such as the shape change of platelets upon stimulation and the reversibility of platelet aggregation cannot be assessed ${ }^{1}$ ), and therefore, these whole blood assays cannot be used to diagnose specific PFD ${ }^{10}$. However, these assays may be used to exclude PFD in a screening setting, as they are rapid and easy to use.

Since patients with PFD suffer from bleeding symptoms and can bleed excessively after surgery and trauma ${ }^{11}$, it is of importance to exclude PFD, to avoid these bleeding risks $^{8}$. A worldwide survey, in which 202 laboratories from 37 countries participated, demonstrated that PFA and Multiplate are used to screen for PFD in 53\% and $12 \%$ of the laboratories, respectively ${ }^{12}$. Previous studies evaluated the screening value of PFA for PFD (as detected by the LTA) and found variable results depending on the severity of PFD ${ }^{6}$. The screening value of Multiplate for PFD has not been studied. We aimed to establish the utility of Multiplate and PFA as screening tests for PFD as detected by 
the gold standard LTA. PFD were defined as having $\geq 2$ LTA curve abnormalities ${ }^{13}$. In addition, we assessed the ability of individual Multiplate agonists and PFA kits to correctly identify the corresponding LTA curve abnormalities. We used data from three different patient cohorts in which we expected differences in prevalence and severity of PFD, including preoperative patients, patients referred to the hematologist for bleeding evaluation, and patients with a diagnosed bleeding disorder.

\section{Methods}

\section{Study design}

Data from three observational studies, which evaluated the diagnostic value of established and new hemostatic tests in patients with a (suspected) bleeding tendency, were used for this analysis. These studies included adult subjects from September 2013 until September 2016 and performed laboratory tests in preoperative patients (PANE study), patients referred to the hematologist for bleeding evaluation, (ProBe-AHP study), and patients with a diagnosed bleeding disorder of any kind (BEPA study). This last group was included to assess whether the tests could discriminate between patients with and without severe PFD. Subjects with platelet count below $100 \times 10^{9} / \mathrm{L}$ and/or hematocrit below $0.25 \mathrm{~L} / \mathrm{L}$, with active bleeding or use of specific medication (e.g. antiplatelet therapy, anticoagulants, non-steroidal antiinflammatory drugs (NSAIDs)) were excluded to avoid interference with test results on LTA, Multiplate and PFA ${ }^{1,2,6,8,14}$. Patients with von Willebrand Factor (VWF) levels below $50 \%$ were excluded for the detection of PFD with PFA, because these patients can have abnormal PFA results (due to von Willebrand disease, VWD) without having a PFD. Detailed study designs are presented in Appendix 3.1.

To assess the performance of Multiplate and PFA as screening tests for PFD, Multiplate and PFA results were compared to the results of LTA as reference test for PFD, within the three study groups. First, the diagnostic value of Multiplate and PFA to detect PFD using previously defined cut-off values was assessed. Second, we assessed the ability of the individual Multiplate agonists and PFA kits to correctly identify the corresponding abnormal LTA agonist curve (e.g. Multiplate ASPItest should detect LTA AA curve abnormality and not LTA TRAP curve abnormality).

Informed consent was obtained from all patients. The studies were approved by the MUMC medical ethical committee. 


\section{Blood sampling}

Patients were asked to avoid fat containing food 4 hours before blood withdrawal, as plasma lipids might interfere with LTA measurements. Patients had to stop any interfering medication 7 days before blood withdrawal. Blood was drawn from the antecubital vein by puncture using a $21 \mathrm{G}$ needle. Complete blood count was measured in EDTA blood ( $1,8 \mathrm{mg} / \mathrm{mL}$, BD Vacutainer, Plymouth), citrated blood was used for the LTA and PFA and Hirudin blood was used for the Multiplate. LTA and Multiplate were performed within 3-4 hours; PFA was performed 1 hour after blood withdrawal. For the preparation of platelet-rich plasma (PRP), citrated-blood was centrifuged at $170 \mathrm{~g}$ for $10 \mathrm{~min}$ at $18^{\circ} \mathrm{C}$. For the preparation of platelet free plasma (PFP), citrated-blood was centrifuged at $2500 \mathrm{~g}$ for $5 \mathrm{~min}$ at $18^{\circ} \mathrm{C}$. The plasma was transferred to another tube and centrifuged at $10000 \mathrm{~g}$ for $10 \mathrm{~min}$ at $18^{\circ} \mathrm{C}$.

Performance of LTA was largely based on Clinical Laboratory Standards Institute (CLSI) and Scientific and Standardization Committee of the International Society of Hemostasis and Thrombosis (ISTH-SSC) guidelines ${ }^{15,16}$. In the ProBe-AHP and BEPA study, platelet count in PRP was adjusted with autologous platelet poor plasma (PPP) to $250 \times 10^{9} / \mathrm{L}$. In the PANE study, the platelet count was not adjusted, unless PRP platelet count was $>600 \times 10^{9} / \mathrm{L}$ (adjusted to $500 \times 10^{9} / \mathrm{L}$ ). Samples from healthy controls were run every 1-2 weeks as an internal quality control for LTA, Multiplate and PFA.

\section{Platelet function tests}

The results of all platelet function tests were interpreted without knowledge of other test results.

\section{Light transmission aggregometry (LTA)}

Blood was collected in 3.2\% sodium citrate Vacuette tubes ( $9 \mathrm{~mL}$; Greiner Bio-One). The Chronolog (Chrono-log Corporation, Havertown) was used for the Probe-AHP study and the BEPA study, the PAR-4 Platelet Aggregometer (Hart Biologicals, Hartlepool) was used for the PANE study. Platelet aggregation in PRP was initiated using ADP (chronology CH384) $5 \mu \mathrm{M}$, ADP $10 \mu \mathrm{M}$, Collagen (COL; Chrono-par Ref385) $1 \mu \mathrm{g} / \mathrm{mL}$ and $4 \mu \mathrm{g} / \mathrm{mL}$, Epinephrine (EPI; chronolog CH393) $10 \mu \mathrm{M}$, Arachidonic acid (AA; Bio/Data) $1 \mathrm{mM}$, Ristocetine (RIST; Chronolog Stago Ref396) $1.5 \mathrm{mg} / \mathrm{ml}$ and thrombin receptor activating peptide (TRAP; Boom H8105) $15 \mu \mathrm{M}$. The agonist concentrations were based upon the CLSI guideline and published literature ${ }^{3,15,17}$. Local within-run coefficient of variation for both the Chronolog and PAR-4 was between $2.2 \%$ and $3.5 \%$. 


\section{Multiplate}

Blood was collected in Hirudin Blood Tubes ( $3 \mathrm{~mL}$, Double Wall; Roche Rotkreuz, Switzerland). Platelet aggregation was measured on the Multiplate Analyzer (Roche, Rotkreuz, Switzerland) and initiated using AA $0.5 \mathrm{mM}$ (ASPItest), TRAP $32 \mu \mathrm{M}$ (TRAPtest), collagen $3.2 \mu \mathrm{g} / \mathrm{ml}$ (COLtest) and ADP $6.4 \mu \mathrm{M}$ (ADPtest). The agonist concentrations were according to the manufacturer leaflet. Test results were expressed as area under the curve (AUC). Reference values were established in our laboratory by analyzing samples of 25 healthy volunteers with EP evaluator software (Data Innovations) ${ }^{18}$. Reference values for AUC were 33-108 for ADP, 50-119 for AA, 70-130 for TRAP and 45-125 for COL. Multiplate was considered 'abnormal' when $\geq 1$ agonist showed low AUC. Local within-run coefficient of variation for all Multiplate agonists was between 8.4 and $9.9 \%$.

\section{PFA}

Blood was collected in $3.2 \%$ sodium citrate tubes (Greiner bio-one). The platelet function analyzer 200 (PFA-200; Innovance PFA-200 System, Siemens, Marburg, Germany) was used. Closure times (CT) were recorded with collagen and epinephrine (C-EPI) or collagen and ADP (C-ADP) kits. Reference intervals for PFA-200 were provided by the manufacturer and verified in our laboratory by analyzing the test results from 23 healthy volunteers with EP evaluator software. PFA was considered 'abnormal' when $\geq 1$ kits showed prolonged CT according to the manufacturer leaflet (>160 seconds for C-EPI, >118 seconds for C-ADP). Local within-run coefficient of variation for both PFA kits was 9.5\%.

\section{Definition of platelet function disorders}

A platelet function defect was considered present in case of deviant LTA curves with $\geq 2$ agonists or when aggregation with ristocetin or collagen $4 \mu \mathrm{g} / \mathrm{mL}$ was the only abnormal curve (Table 3.1). The aggregation curves of LTA were visually assessed by a senior technician, a hematologist and a clinical chemist. A deviant LTA curve was defined in case of maximal aggregation below $60 \%$; reversibility to below $50 \%$ of the maximal aggregation; or if maximal aggregation was not reached within 3 minutes (visually interpreted as delayed). A delayed aggregation with epinephrine was not considered abnormal, as this is very often present in healthy subjects ${ }^{2,6,16}$.

\section{Statistical analysis}

Continuous variables are expressed as mean with standard deviation (SD) for normally distributed variables or median with interquartile range (IQR) otherwise. Categorical variables are expressed as counts and percentages. To assess whether Multiplate and 
PFA are able to detect PFD ( $\geq 2$ abnormal LTA agonists), the diagnostic performance of Multiplate and PFA was assessed by calculating sensitivity and specificity with $95 \%$ confidence intervals, using previously proposed cut-off values. To assess whether individual Multiplate agonists and PFA kits can detect corresponding LTA curve abnormalities, the different Multiplate agonists and PFA kits were compared to the corresponding LTA agonists. Their diagnostic performance was assessed by construction of receiver operating characteristic (ROC) curves, which visualize the trade-off between sensitivity and specificity over a range of cut-off points for positive test results. Areas under the ROC curves (AUC) with 95\% confidence intervals were calculated. Statistical analyses were performed with IBM SPSS statistics version 24.0; significance was assumed at $p<0.05$.

Table 3.1 Definitions of platelet function disorders (LTA) and Multiplate/PFA abnormalities.

\begin{tabular}{|c|c|c|c|}
\hline & $\begin{array}{l}\text { Gold standard: LTA (AA, } \\
\text { TRAP, ADP, COL, EPI, RIST) }\end{array}$ & $\begin{array}{l}\text { Screening test: Multiplate } \\
\text { (ASPItest, TRAPtest, } \\
\text { ADPtest, COLtest) }\end{array}$ & $\begin{array}{l}\text { Screening test: PFA-200 } \\
\text { (C-EPI and C-ADP) }\end{array}$ \\
\hline $\begin{array}{l}\text { Platelet function } \\
\text { defect/abnormal test } \\
\text { result }\end{array}$ & $\begin{array}{l}\geq 2 \text { agonist abnormalities, or } \\
\text { only COL4/RIST* abnormal }\end{array}$ & $\geq 1$ agonist abnormalities & $\begin{array}{l}\text { at least one kit } \\
\text { prolonged }\end{array}$ \\
\hline $\begin{array}{l}\text { No platelet function } \\
\text { defect/normal test } \\
\text { result }\end{array}$ & $\begin{array}{l}<2 \text { agonist abnormalities } \\
\text { (except for only COL4/RIST*) }\end{array}$ & no agonist abnormality & no kit prolonged \\
\hline
\end{tabular}

Definition of platelet function disorders according LTA and Multiplate/PFA abnormalities. The diagnostic performance of the screening tests Multiplate and PFA was calculated, using these predefined cut-off levels.

* For comparison LTA-Multiplate patients with only LTA ristocetin abnormalities were not considered, as GP1b function cannot be assessed by current Multiplate agonists. AA, arachidonic acid; ADP, adenosine diphosphate; AUC, area under the curve; COL, collagen; $C T$, closure time; EPI, epinephrine; LTA, light transmission aggregometry; PFA, platelet function analyzer; TRAP, thrombin receptor activatable peptide; RIST, ristocetin.

\section{Results}

\section{Study population}

The three studies included 414 patients: 335 preoperative patients, 54 patients referred to the hematologist for bleeding evaluation and 25 patients previously diagnosed with a bleeding disorder. This latter group included $3(12 \%)$ patients with Glanzmann thrombasthenia (GT), a severe platelet function disorder ${ }^{10,17,18}$. The remaining patients had other diagnoses, such as VWD type 1 (3), VWD type 2 (5), hemophilia A (7) or hemophilia B (1), carriership of hemophilia A (2) or hemophilia B (2), factor XI deficiency (1) and factor XII deficiency (1). Patients with VWF $<50 \%$ were 
excluded from LTA-PFA comparison. Not all tests were successfully accomplished in all patients due to partially failed blood withdrawal or laboratory errors. Figure 3.1 shows the number of platelet function test results that were available for the analyses.

Table 3.2 shows the baseline characteristics of all patients. LTA detected a PFD in $16(5 \%)$ preoperative patients and in 10 (19\%) patients referred for bleeding evaluation. All PFD that were identified in these patients were 'thrombopathy not otherwise specified (NOS)', usually classified as mild PFD ${ }^{10,19,20}$. In the group of patients with a previously diagnosed bleeding disorder, LTA abnormalities specific for GT were identified in all three patients with GT (LTA detection rate of 100\%). In the other patients, as suspected, no LTA abnormalities were found. Details on PFD as detected by the LTA are shown in Appendix 3.2.

Figure 3.1 Number of platelet function tests available for analysis.

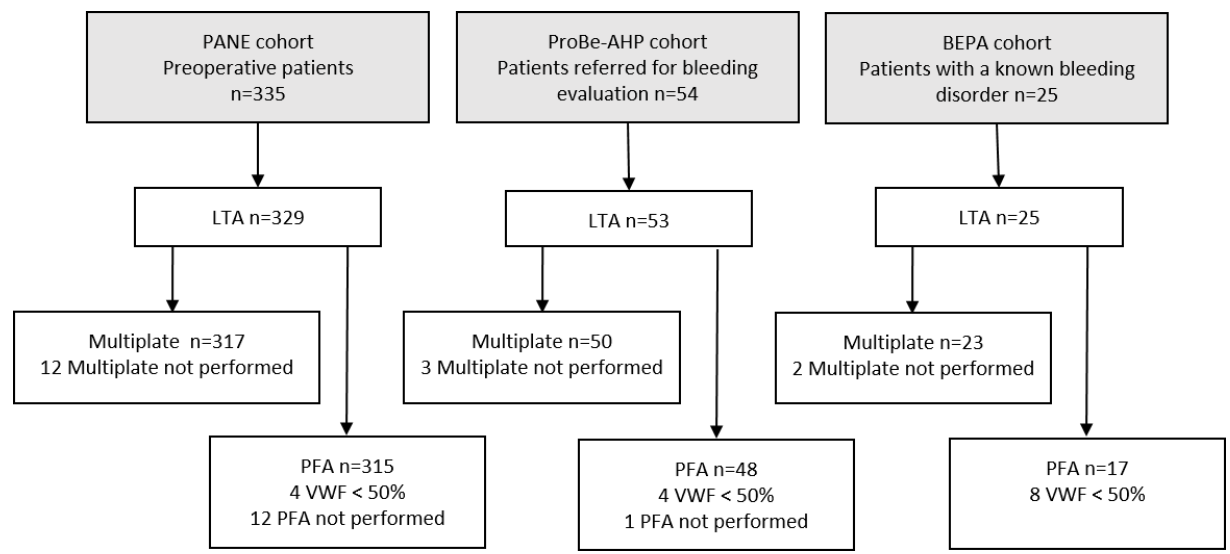

This figure shows the number of platelet function test results that were available for the LTA-Multiplate and LTA-PFA comparison. PFA, platelet function analyser; LTA, light transmission aggregometry; VWF, von Willebrand factor. 
Table 3.2 Patient characteristics.

\begin{tabular}{lccc}
\hline $\mathrm{n}=414$ & $\begin{array}{c}\text { Preoperative patients } \\
\mathrm{n}(\%), \text { mean } \pm \text { SD }\end{array}$ & $\begin{array}{c}\text { Patients referred for } \\
\text { bleeding evaluation } \mathrm{n}(\%), \\
\text { mean } \pm \text { SD }\end{array}$ & $\begin{array}{c}\text { Patients with a diagnosed } \\
\text { bleeding disorder } \mathrm{n}(\%), \\
\text { mean } \pm \text { SD }\end{array}$ \\
\hline Number & 335 & 54 & 25 \\
Age & $52 \pm 15$ & $46 \pm 16$ & $50 \pm 13$ \\
Female & $216(65)$ & $41(76)$ & $11(44)$ \\
Hematocrit (L/L) & & & \\
Women & $0.41 \pm 0.03$ & $0.42 \pm 0.03$ & $0.43 \pm 0.04$ \\
Men & $0.45 \pm 0.03$ & $0.44 \pm 0.05$ & $0.45 \pm 0.04$ \\
Platelets (10\%) & $270 \pm 64$ & $269 \pm 73$ & $239 \pm 64$ \\
MPV & $10.6 \pm 0.9$ & $10.5 \pm 1.1$ & $10.4 \pm 1.1$ \\
PFD & $16(5)$ & $10(19)$ & $3(12)$ \\
\hline
\end{tabular}

MPV, mean platelet volume; LTA, light transmission aggregometry; PFD, platelet function disorder; SD, standard deviation.

\section{Test performance of Multiplate and PFA for the detection of PFD}

To assess whether Multiplate and PFA can detect PFD ( $\geq 2$ abnormal LTA agonists), the diagnostic performance of Multiplate and PFA was assessed by calculating sensitivity and specificity as shown in Table 3.3. For these calculations, predefined cut-off values of Multiplate and PFA were used. In preoperative patients and patients referred for bleeding evaluation, sensitivity of both the Multiplate and PFA was low for the detection of PFD (0-40\%) but specificity was high (82-97\%). In patients with a diagnosed bleeding disorder, both the Multiplate and PFA showed abnormalities compatible with GT, meaning that the sensitivity was $100 \%$. Specificity was also high for both the Multiplate and PFA (100 resp. 79\%).

Table 3.3 Diagnostic performance of Multiplate and PFA for the detection of PFD.

\begin{tabular}{lrrrrrr}
\hline & TP & FP & FN & TN & Sensitivity (95\%Cl) & Specificity (95\%Cl) \\
\hline $\begin{array}{l}\text { Multiplate } \\
\text { Preoperative patients }\end{array}$ & 1 & 9 & 15 & 292 & $0.06(0.00-0.30)$ & $0.97(0.94-0.99)$ \\
$\begin{array}{l}\text { Patients referred for evaluation } \\
\text { Patients with bleeding disorder }\end{array}$ & 4 & 1 & 6 & 39 & $0.40(0.12-0.74)$ & $0.97(0.87-1.00)$ \\
PFA & & 0 & 0 & 20 & $1.00(0.29-1.00)$ & $1.00(0.83-1.00)$ \\
Preoperative patients & 0 & 33 & 16 & 266 & $0.00(0.00-0.21)$ & $0.89(0.85-0.92)$ \\
Patients referred for evaluation & 2 & 7 & 7 & 32 & $0.22(0.03-0.60)$ & $0.82(0.66-0.92)$ \\
Patients with bleeding disorder & 3 & 3 & 0 & 11 & $1.00(0.29-1.00)$ & $0.79(0.49-0.95)$ \\
\hline
\end{tabular}

Sensitivity and specificity of the Multiplate and PFA when using predefined cut-off levels in 3 patient groups, using the presence of PFD as detected by the LTA as reference. $\mathrm{Cl}$, confidence interval; $\mathrm{FN}$, false negative; FP, false positive; PFA, platelet function analyzer; TN, true negative; TP, true positive. 


\section{Test performance of Multiplate agonists and PFA kits for detection of LTA curve abnormalities}

To assess whether individual Multiplate agonists and PFA kits can detect corresponding LTA curve abnormalities, ROC curve analyses were performed. These results are shown in Tables 3.4 and 3.5. In preoperative patients and patients referred for bleeding evaluation, the AUCs of all Multiplate agonists and PFA kits varied around 0.50 (between 0.36 and 0.63$)$ except for Multiplate ASPI with AUC $=0.71(95 \% \mathrm{CI}$ 0.49-0.92) in preoperative patients and Multiplate TRAP with $A U C=0.72(95 \% \mathrm{Cl}$ $0.45-0.99$ ) in referred patients. This means that in these patient groups, the individual Multiplate agonists and PFA kits were not or poorly able to differentiate between patients with and without corresponding LTA curve abnormalities. In patients with a previously diagnosed bleeding disorder AUCs of 0.80 and higher, which significantly exceeded the non-diagnostic value of 0.50 , were found for all Multiplate agonists and PFA kits, except for Multiplate $\mathrm{COL}$ with AUC of $0.70(95 \% \mathrm{Cl} 0.38-1.00)$ and PFA-EPI with AUC of 0.74 (95\% Cl 0.33-1.00).

\section{Discussion}

We evaluated the diagnostic performance of Multiplate and PFA as screenings tests for PFD and LTA aggregation curve abnormalities in different patient populations. In both preoperative patients and patients referred for bleeding evaluation, only mild platelet function disorders were found by the LTA. The sensitivity for detection of mild PFD ( $\geq 2$ abnormal LTA agonists) was low (0-40\%) at the pre-defined, conventionally used, cut-off values. In addition, the individual Multiplate agonists and PFA kits were not, or only poorly able to identify corresponding LTA agonist abnormalities. However, for the detection of Glanzmann thrombasthenia, which was present in the patient group with previously diagnosed bleeding disorders, the sensitivity of both Multiplate and PFA was high (79-100\%) and both tests discriminated well between patients with and without GT. This suggests that Multiplate and PFA may be used to rule out severe PFD like GT, but cannot be used as screening test to rule out mild PFD.

This is the first study which directly evaluates the clinical utility of Multiplate as a screening test for mild PFD in preoperative patients and patients referred for bleeding evaluation. Two previous studies compared LTA results with Multiplate in a total of 24 patients with GT. Also in these studies, all patients with GT were detected by Multiplate ${ }^{7,21}$. 

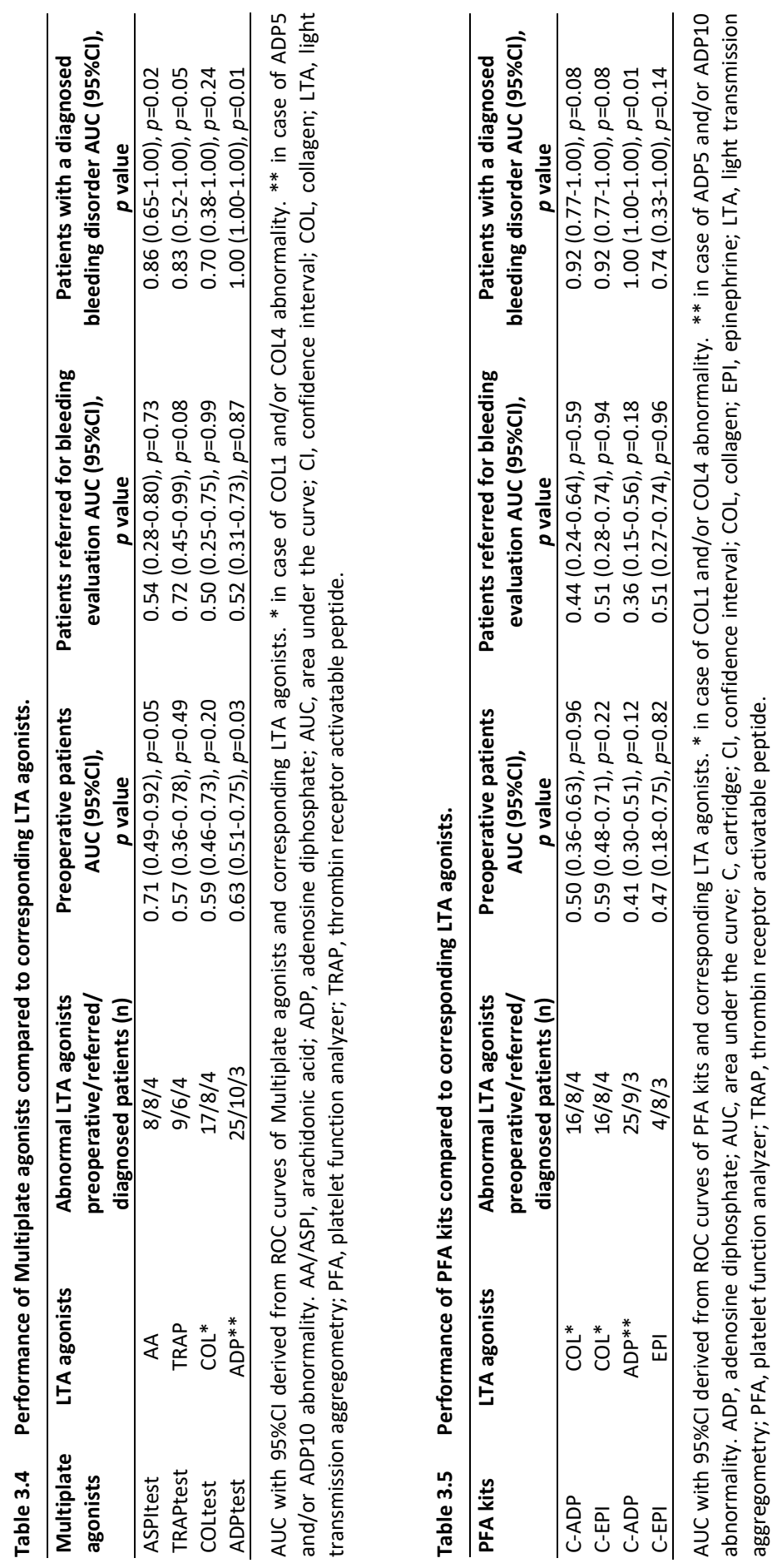
A small number of prospective studies investigated the value of the PFA as a screening test for mild PFD in patients referred for bleeding evaluation. These studies show that the overall sensitivity of the PFA for mild PFD is poor ${ }^{22-24}$. In addition, many reviews as well as a recent guideline from the Platelet Physiology Subcommittee of the ISTH ${ }^{25}$ do not recommended PFA as a screening tool for $\mathrm{PFD}^{2,6,9,10,19,26-28}$. In the preoperative setting, to the best of our knowledge, no previous studies consistently related PFA outcomes to PFD as detected with LTA. In patients with diagnosed bleeding disorders, the value of the PFA was assessed by several studies. These studies predominantly included patients with severe PFD. In agreement with our findings, these studies reported a sensitivity of $80 \%$ and higher ${ }^{29-31}$.

Although the survey by Gresele et al. ${ }^{12}$ showed that Multiplate and PFA are often used in clinical practice to screen for PFD, our results suggest that this practice is obsolete. Multiplate and PFA are not able to exclude the presence of mild PFD (low sensitivity) and LTA curve abnormalities, but they might be able to exclude more severe platelet function disorders. However, severe PFD are unlikely to be encountered in preoperative patients and adult patients referred for bleeding evaluation, as demonstrated by our findings. The main reason for this is that patients with a severe PFD often undergo elaborate hemostatic diagnostic tests in childhood, including LTA, as these patients present early in life with a severe bleeding phenotype or are family members of a proband. However, if LTA testing is not possible, or when there is only a small amount of blood available (e.g. in children), Multiplate and PFA might be an attractive alternative for LTA to exclude GT and possibly other severe PFD.

Our study has some limitations. Although we included in total 414 patients, only 3 patients with a severe PFD were included, therefore we cannot draw firm conclusions on the value of these tests to detect severe PFD. Furthermore, different LTA procedures regarding platelet count adjustment were used for the different studies. The LTA measurements of the ProBe-AHP and BEPA studies were performed in our clinical laboratory, for which the procedure is to adjust the platelet count in PRP (CLSI guideline $\left.{ }^{15}\right)$. The LTA measurements of the PANE study were performed in our research laboratory were non-adjustment of the platelet count in PRP is the procedure (ISTH-SCC guideline ${ }^{16}$ ). Results of a study by Castilloux et al. ${ }^{32}$ showed that there is no difference in clinical diagnosis when adjusting is compared to nonadjusting.

Finally, we recognize that the LTA may not be the ultimate gold standard test for platelet function disorders. Tests like flow-cytometry, transmission electron microscopy or genetic tests might detect a PFD in patients with a normal LTA ${ }^{25}$. In 
addition, these tests may have been able to diagnose the exact platelet function defect in some of our patients with 'thrombopathy not otherwise specified'.

\section{Conclusion}

In preoperative patients and patients referred for bleeding evaluation, Multiplate and PFA were not able to discriminate between patients with and without mild PFD and aggregation curve abnormalities as detected by LTA. This means that they cannot be used as screening tests to rule out mild PFD in these populations. The Multiplate and PFA could only discriminate between patients with and without Glanzmann Thrombasthenia. 


\section{References}

1. Paniccia R, et al. Platelet function tests: a comparative review. Vasc Health Risk Manag 2015;11: 133-48.

2. Hayward CPM. Diagnostic approach to platelet function disorders. Transfus Apher Sci 2008;38(1): 65-76.

3. Dawood BB, et al. Evaluation of participants with suspected heritable platelet function disorders including recommendation and validation of a streamlined agonist panel. Blood 2012;120(25):5041-9.

4. Cattaneo $\mathrm{M}$, et al. Results of a worldwide survey on the assessment of platelet function by light transmission aggregometry: a report from the platelet physiology subcommittee of the SSC of the ISTH. J Thromb Haemost 2009;7(6):1029.

5. Cattaneo M. Light transmission aggregometry and ATP release for the diagnostic assessment of platelet function. Semin Thromb Hemost 2009;35(2):158-67.

6. Harrison $\mathrm{P}$, et al. Guidelines for the laboratory investigation of heritable disorders of platelet function. Br J Haematol 2011;155(1):30-44.

7. Albanyan A, et al. Diagnosis of Glanzmann thrombasthenia by whole blood impedance analyzer (MEA) vs. light transmission aggregometry. Int J Lab Hematol 2015;37(4):503-8.

8. Görlinger K, et al. Perioperative Coagulation Management and Control of Platelet Transfusion by Point-of-Care Platelet Function Analysis. Transfusion Medicine and Hemotherapy 2007;34(6):396-411.

9. Harrison P. The role of PFA-100 testing in the investigation and management of haemostatic defects in children and adults. BJH 2005;130(1):3-10.

10. Podda G, Femia EA, Cattaneo M. Current and emerging approaches for evaluating platelet disorders. Int J Lab Hematol 2016;38 Suppl 1:50-8.

11. Orsini $\mathrm{S}$, et al. Bleeding risk of surgery and its prevention in patients with inherited platelet disorders. Haematologica 2017;102(7):1192-203.

12. Gresele P, et al. Diagnosis of suspected inherited platelet function disorders: results of a worldwide survey. J Thromb Haemost 2014;12(9):1562-9.

13. Hayward CPM, et al. Development of North American Consensus Guidelines for Medical Laboratories That Perform and Interpret Platelet Function Testing Using Light Transmission Aggregometry. Am J Clin Pathol 2010;134(6):955-63.

14. Kuiper $\mathrm{G}$, et al. The use of regression analysis in determining reference intervals for low hematocrit and thrombocyte count in multiple electrode aggregometry and platelet function analyzer 100 testing of platelet function. Platelets 2017;28(7):668-75.

15. Christie DJ, et alL. Platelet function testing by aggregometry; approved guideline. Clinical and Laboratory Standards Institute, Wayne, PA, USA, 2008. Vol. 28 No. 31.

16. Cattaneo $\mathrm{M}$, et al. Recommendations for the Standardization of Light Transmission Aggregometry: A Consensus of the Working Party from the Platelet Physiology Subcommittee of SSC/ISTH. J Thromb Haemost, 2013.

17. Moffat KA, et al. Variability in clinical laboratory practice in testing for disorders of platelet function: results of two surveys of the North American Specialized Coagulation Laboratory Association. Thromb Haemost 2005;93(3):549-53.

18. EP Evaluator product information available on http://datainnovations.com/ep-evaluator.

19. Norman JE, et al. How should we test for nonsevere heritable platelet function disorders? Int J Lab Hematol 2014;36(3):326-33.

20. Nurden AT, Nurden P. Congenital platelet disorders and understanding of platelet function. $\mathrm{Br} \mathrm{J}$ Haematol 2014;165(2):165-78.

21. Awidi $A$, et al. Comparison of platelet aggregation using light transmission and multiple electrode aggregometry in Glanzmann thrombasthenia. Platelets 2009;20(5):297-301.

22. Quiroga T, et al. Template bleeding time and PFA-100 have low sensitivity to screen patients with hereditary mucocutaneous hemorrhages: comparative study in 148 patients. J Thromb Haemost 2004;2(6):892-8.

23. Cattaneo M. Are the bleeding time and PFA-100R useful in the initial screening of patients with mucocutaneous bleedings of hereditary nature? J Thromb Haemost 2004;2(6):890-1. 
24. Podda GM, et al. Usefulness of PFA-100 (R) testing in the diagnostic screening of patients with suspected abnormalities of hemostasis: comparison with the bleeding time. J Thromb Haemost 2007;5(12):2393-8.

25. Gresele $P$, Diagnosis of inherited platelet function disorders: guidance from the SSC of the ISTH. J Thromb Haemost 2015;13(2):314-22.

26. Hayward CPM, et al. Platelet function analyzer (PFA)-100 closure time in the evaluation of platelet disorders and platelet function. J Thromb Haemost 2006;4(2):312-9.

27. Hayward CP. Diagnostic evaluation of platelet function disorders. Blood Rev 2011;25(4):169-73.

28. Favaloro EJ. Clinical utility of the PFA-100. Semin Thromb Hemost 2008;34(8):709-33.

29. Cattaneo $\mathrm{M}$, et al. Evaluation of the PFA-100 system in the diagnosis and therapeutic monitoring of patients with von Willebrand disease. Thromb Haemost 1999;82(1):35-9.

30. Posan E, et al. Comparison of PFA-100 testing and bleeding time for detecting platelet hypofunction and von Willebrand disease in clinical practice. Thromb Haemost 2003;90(3):483-90.

31. Mammen EF, et al. PFA-100 (TM) system: A new method for assessment of platelet dysfunction. Semin Thromb Hemost 1998;24(2):195-202.

32. Castilloux JF, et al. A prospective cohort study of light transmission platelet aggregometry for bleeding disorders: is testing native platelet-rich plasma non-inferior to testing platelet count adjusted samples? Thromb Haemost 2011;106(4):675-82. 


\section{Appendix 3.A Study protocol summaries}

\section{PANE study}

Title: Preoperative screening for mild bleeding disorders

Acronym: PANE study

Trial registry: Nederlands Trial Register, NTR4070.

METC (medical ethical committee): NL38767.068.11, approved July 26, 2013.

Principal Investigator: Prof. dr. ir. Y.M.C. Henskens, Maastricht University Medical Center+, P. Debeyelaan 25, 6202 AZ, Maastricht.

Study type: observational - diagnostic.

Study objectives: to assess the prevalence of haemostatic abnormalities in patients with and without reported bleeding symptoms on a preoperative questionnaire, consisting of guideline-proposed bleeding questions, and appraise the diagnostic value of several screening modalities for the identification of patients with haemostatic abnormalities

Study design: Patients reporting bleeding symptoms on the preoperative questionnaire are primarily included. A random sample of patients not reporting bleeding symptoms is also included. Preoperatively, all patients undergo haemostatic testing and complete the International Society of Thrombosis and HaemostasisBleeding Assessment Tool (ISTH BAT).

Study population: adults undergoing any kind of surgery.

Inclusion criteria: Age $\geq 18$ years; undergoing elective surgery; signed informed consent.

Exclusion criteria: pregnancy; the use of medication which may interfere with diagnostic tests; anemia and thrombocytopenia.

Primary endpoint: the prevalence of haemostatic abnormalities, defined as coagulation or fibrinolysis factor levels below the reference range and platelet function defects.

Secondary endpoint: diagnostic value of screening assays for haemostatic disorders, using gold standard assays as reference. 


\section{ProBe-AHP study}

Title: Predictors of Bleeding Evaluation in Adult Hematologic Patients with Bleeding Tendencies

Acronym: ProBe-AHP study.

Other ID: the Bloody study.

Trial registry: Nederlands Trial Register, NL9643.

METC (medical ethical committee): 14-4-036 , approved on 14 August 2014

Principal Investigator: Prof. dr. E.A.M. Beckers, Maastricht University Medical Centert, P. Debeyelaan 25, 6202 AZ, Maastricht.

Study type: observational - diagnostic.

Study objectives: to assess the diagnostic parameters of experimental hemostatic tests (e.g. impedance aggregometry, rotational thromboelastometry analysis) by comparing them to the standard diagnostic algorithm and laboratory tests used in MUMC+ for the work-up of patients with a bleeding tendency.

Study design: patients with (suspected) bleeding tendencies are investigated on an out-patient clinic basis, according to the standard work-up of Maastricht University Medical Center+. According to this work-up patients are assessed by a quantitative bleeding questionnaire and screening and additional blood tests are done. In addition, extra blood will be drawn for experimental tests. Patient and treating hematologist are blinded to the results of the experimental tests.

Patient population: out-of-clinic patients referred for evaluation of bleeding tendency.

Inclusion criteria: Age $\geq 18$ years; signed informed consent; patients with (suspected) bleeding tendency.

Exclusion criteria: pregnancy (or lactating); active bleeding due to medical interventions or surgical/obstetrical causes; the use of medication which may interfere with diagnostic tests.

Primary endpoint: sensitivity, specificity, negative predictive value, positive predictive value, likelihood ratio of the experimental hemostatic tests. 


\section{BEPA study}

Title: Predictors of Bleeding Evaluation in Adult Hematologic Patients with Bleeding Tendencies. Patients with established bleeding disorders: The BePa verification study Acronym: The BEPA study.

Trial registry: Nederlands Trial Register, NL9644.

METC (medical ethical committee): NL51315.068.14, approved on 18 May 2015

Principal Investigator: Prof. dr. E.A.M. Beckers, Maastricht University Medical Center+, P. Debeyelaan 25, 6202 AZ, Maastricht.

Study type: diagnostic-validation.

Study objectives: To evaluate the diagnostic accuracy of the experimental tests (e.g. impedance aggregometry, rotational thromboelastometry analysis) to detect bleeding disorders and to evaluate whether or not they can be used for monitoring the effects of coagulant factor replacement therapy.

Study design: In this diagnostic study we will evaluate if the experimental tests and the bleeding assessment tool (BAT) are able to detect bleeding disorders. Sensitivity and specificity of the experimental tests will be estimated at various cut-off values and results will be summarized in receiver operating characteristic (ROC) curves with corresponding area under the curve (AUC). Values of the experimental in patients with a bleeding disorder before and after planned medical intervention will be evaluated to see if these tests are able to detect the differences in plasma factor levels before and after treatment (e.g. rotational thromboelastometry analysis).

Patient population: Registered patients with established bleeding disorders recruited from the hemophilia treating center MUMC+.

Inclusion criteria: age $\geq 18$ years; signed informed consent.

Exclusion criteria: pregnancy (or lactating); current active bleeding due to medical interventions or surgical/obstetric causes; use of any interfering medication $<48$ hours before laboratory testing; known platelet level lower than 100,000/ $\mu$ l, known hematocrit lower than $30 \%$.

Primary endpoint: Diagnostic value (sensitivity, specificity, negative and positive predictive value) of the experimental haemostatic tests, using gold standard assays as reference. 
Appendix 3.B Platelet function defects detected by LTA

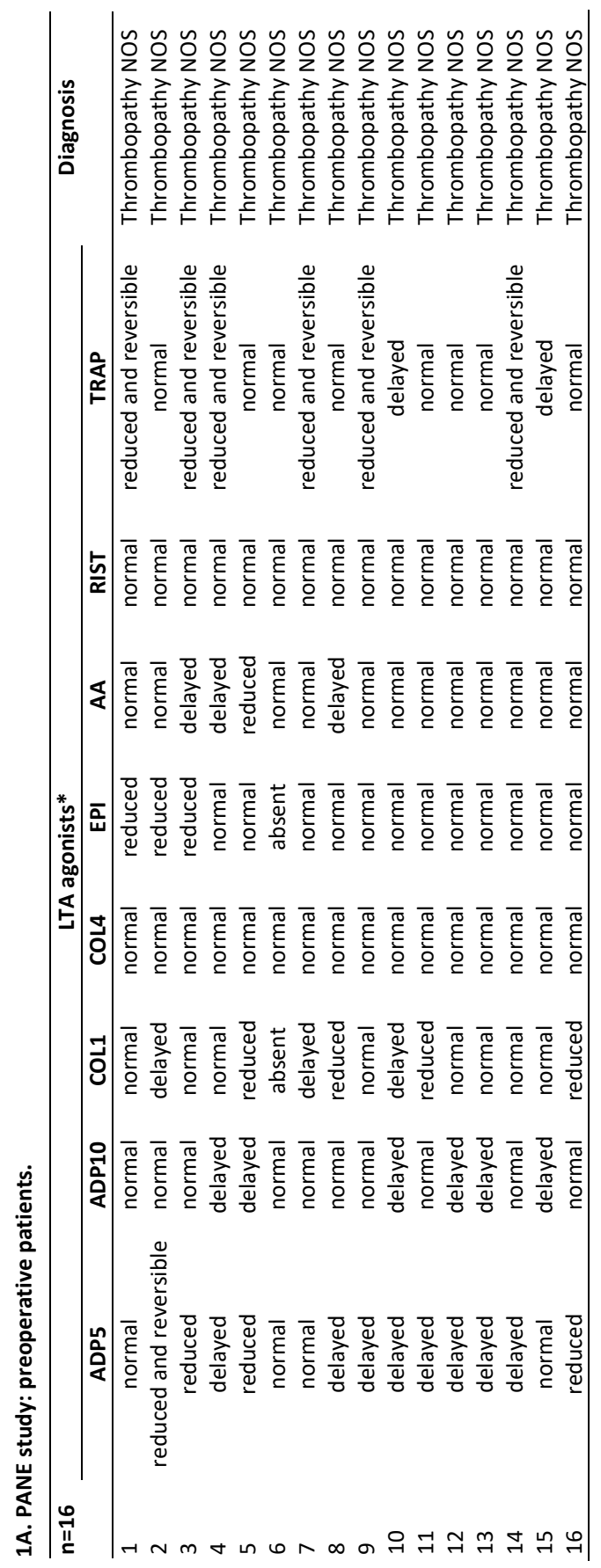



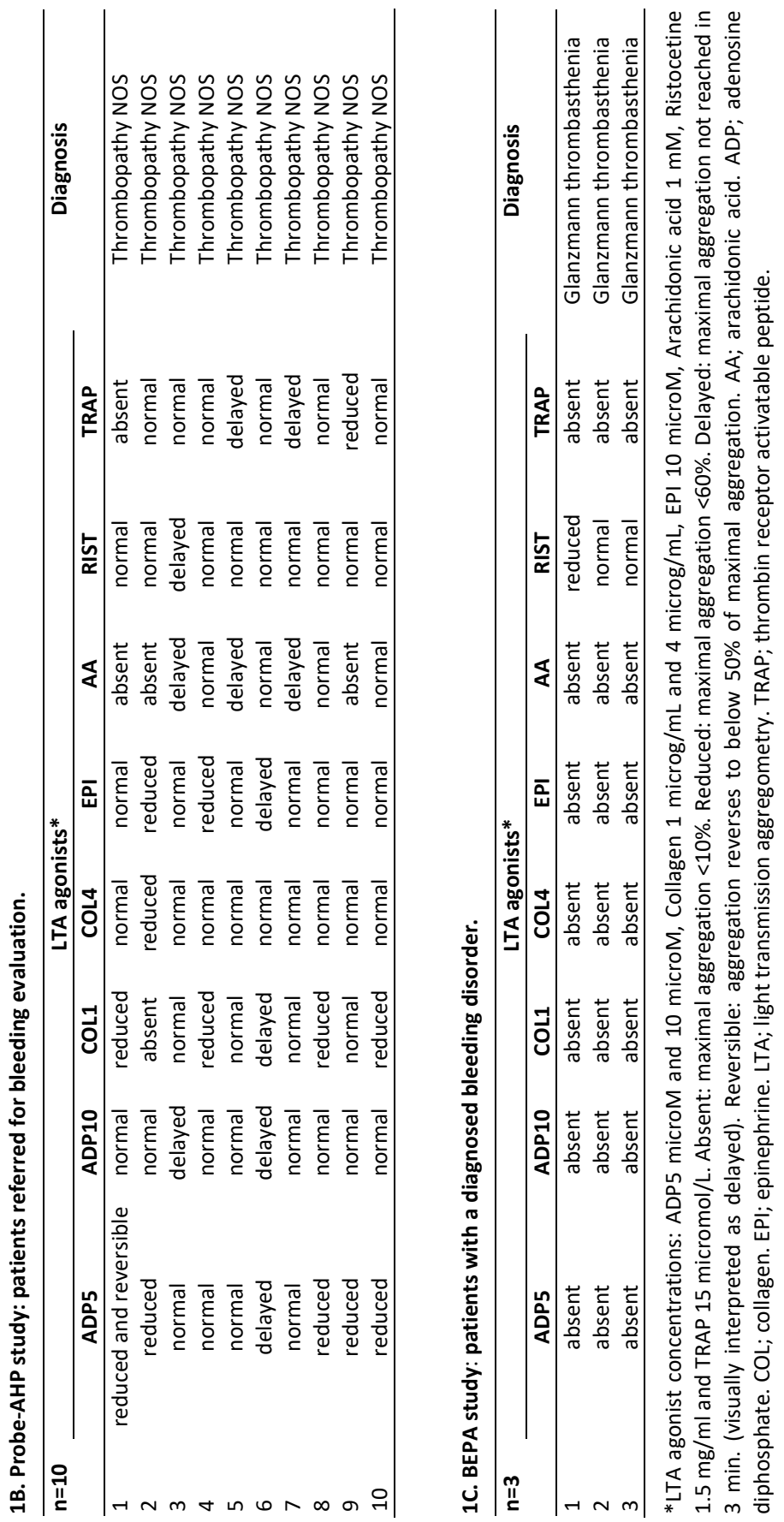



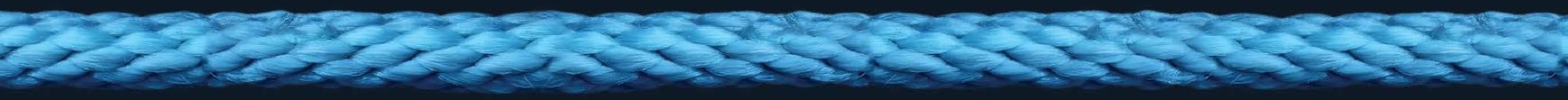




\section{CHAPTER 4}

Multiparameter platelet function analysis of bleeding patients with a prolonged platelet function analyzer closure time

Floor C.J.I. Heubel-Moenen, Sanne L.N. Brouns, Linda Herfs, Lara S. Boerenkamp, Rick J.H. Wetzels, Paul W.M. Verhezen, Patric Machiels, Karyn Megy, Kate Downes, Johan W.M. Heemskerk, Erik A.M. Beckers, Yvonne M.C. Henskens

Br J Haematol (accepted for publication) 


\section{Abstract}

\section{Introduction}

Patients referred for evaluation of bleeding symptoms occasionally have a prolonged platelet function analyzer (PFA) closure time, without evidence for von Willebrand disease or impaired platelet aggregation.

\section{Aim}

To establish a shear-dependent platelet function defect in bleeding patients with a prolonged PFA closure time as single aberrant test.

\section{Methods}

Patients were included based on high bleeding score and prior PFA prolongation. Common tests of VWF and platelet function and exome sequencing were performed. Microfluidic analysis of shear-dependent collagen-induced whole-blood thrombus formation at $37^{\circ} \mathrm{C}$ was performed to inform on shear-dependent platelet adhesion, activation and aggregation.

\section{Results}

In 14 PFA-only patients, compared to healthy volunteers, microfluidic tests showed significant lower platelet adhesion ( 49 vs. $61 \%, p<0.001$ ) and thrombus formation parameters $(p<0.008)$. This was accompanied by lower integrin activation, phosphatidylserine exposure and P-selectin expression. Principal component analysis indicated VWF as primary explaining variable of PFA prolongation, whereas conventional platelet aggregation primarily explained the reduced thrombus parameters under shear. In five patients with severe microfluidic abnormalities, conventional platelet aggregation was in the lowest range of normal. No causal variants in Mendellian genes known to cause bleeding or platelet disorders were identified.

\section{Conclusion}

These results show that an abnormal PFA as a single aberrant test in bleeding patients should be further evaluated. Multiparameter assessment of whole-blood thrombus formation under shear senses single or combined effects of low-normal VWF and lownormal platelet aggregation in these patients, suggesting a shear-dependent platelet function defect, not detected by static conventional hemostatic tests. 


\section{Introduction}

Whole-blood assessment using a platelet function analyzer (PFA) is regularly used in the diagnostic work-up of patients suspected of a bleeding disorder. The PFA test has been developed as a proxy measurement of primary hemostasis under conditions of high wall-shear rate ${ }^{1,2}$. High-shear blood flow is created by a pressure gradient over a membrane with a narrow central aperture, which is coated with collagen and either epinephrine (EPI) or adenosine diphosphate (ADP). A platelet aggregate or plug is formed that gradually closes the aperture, thus resulting in an aperture closure time (CT), which is recorded ${ }^{2,3}$. However, use of the PFA test in identifying patients with a bleeding disorder has been questioned in single studies and meta-analyses ${ }^{2-5}$.

As accepted, the PFA is particularly sensitive in the detection of von Willebrand disease (VWD), especially when the level of von Willebrand factor (VWF) is below $30 \%{ }^{6-8}$. Similarly, in such patients, the PFA is used for therapeutic monitoring of the effects of desmopressin or VWF supplementation ${ }^{9-11}$. Furthermore, the PFA is sensitive for severe platelet function disorders (PFD), such as Glanzmann's thrombasthenia (GT) and Bernard-Soulier syndrome (BSS) ${ }^{12-14}$. However, this test has only limited sensitivity for diseases with milder bleeding phenotype ${ }^{15}$, like primary platelet secretion syndrome, storage pool disease ${ }^{16-18}$ or moderately low levels of VWF $(30-50 \%)^{19}$. Accordingly, in the diagnostic work-up for patients with bleeding symptoms, the finding of a prolonged PFA indicates that further diagnostic testing is required ${ }^{3,13,20}$. Often extensive laboratory testing is required before a final diagnosis can be made.

For identification of platelet function disorders, light transmission aggregometry (LTA) is the gold standard, often in combination with ATP release or flow cytometry $^{21-23}$. For the diagnosis of VWD, plasma levels of VWF activity and antigen are determined, if indicated, in combination with testing of VWF multimer pattern, VWF-collagen binding and VWF-factor VIII binding ${ }^{24}$. Several reports indicate that, even after extensive testing, in a significant proportion of patients with bleeding symptoms, an 'unexplained' prolonged PFA-CT is the only abnormality found $d^{25,26}$. These 'PFA-only' patients are, like other patients with bleeding of unknown cause, provided with a treatment plan, e.g. receiving desmopressin or tranexamic acid, to prevent excessive bleeding upon surgery or tooth extraction ${ }^{27,28}$.

An explanation for a prolonged PFA-CT, as single laboratory aberration, is that this is the only test in the diagnostic work-up for bleeding evaluation that relies on highshear forces. In primary hemostasis, initial platelet rolling is regulated by the interaction of platelet GPIb-V-IX complex to VWF bound to collagen. High shear is required to unfold VWF multimers to expose the VWF-A1 binding site for platelet 
$\mathrm{GPIb}^{29}$. Accordingly, shear-dependent defects of both platelets and VWF will contribute to abnormal PFA-CT values, which are likely not detected in static tests of hemostasis. A clear limitation of the PFA however, is that it only gives the aperture closure time as a test result, not providing any information about the cause of a prolonged CT.

Microfluidic whole blood flow assays are frequently used to study platelet thrombus formation in vitro. As we and others have shown previously, multiparameter measurements of thrombus formation give a wealth of information about platelet adhesion, activation and aggregation under flow conditions, especially when combined with arrays of microspots in the same flow chamber ${ }^{29,30}$. Thus, when using microspots of collagen-I and collagen-III (both binding plasma VWF), multiple qualitative and quantitative platelet traits have been identified, linked to genetic variation of the collagen receptor GPVI and to inherited bleeding disorders ${ }^{31-33}$. In combination with the use of brightfield and multicolor fluorescence microscopy, the microfluidic assay thus produces multiple platelet-dependent outcome values relevant for flow conditions ${ }^{34-36}$.

For the present study, our aim was to investigate whether and how multiparameter shear-dependent microfluidic testing operating at physiological temperature can help to identify abnormalities in platelet function or VWF that explain the prolonged closure times of PFA-only patients with a bleeding history. Furthermore, patients DNA was sequenced using targeted high throughput gene panel testing to identify genetic variants known to cause bleeding and platelet disorders.

\section{Methods}

A detailed description of the methods is available in Appendix 4.A.

\section{Study population}

Patients were selected from three observational cohort studies including adult subjects between September 2013 and November 2020. In all cases, a large panel of in-hospital laboratory tests was performed evaluating the blood from: (i) 136 patients referred to the hematologist for bleeding evaluation (ProBe-AHP cohort), (ii) 240 patients examined pre-operative (PANE cohort), and (iii) 49 patients with a prior diagnosed bleeding disorder of any kind (BEPA cohort). Detailed study designs were described previously ${ }^{37}$ in Appendix 3.A.

For the present paper, patients were included if prior PFA results were abnormal (i.e. one or both cartridges showing a prolonged CT) and if this was the only 
aberration found in the diagnostic work-up. Four patients with a known bleeding disorder were selected from the BEPA study as positive controls. For the microfluidic assay, blood samples from 50 healthy controls were evaluated. All studies were approved by the local medical ethics committee. All participating patients and healthy individuals gave written informed consent according to the Helsinki declaration.

\section{Data collection and prospective laboratory evaluation}

For all patients, the following data was retrospectively collected: medical history; family history; medication use; international society on thrombosis and hemostasisbleeding assessment tool (ISTH BAT) score; diagnosis; complete blood counts; PFA results; LTA results; VWF activity and antigen. Prospectively, medical history; ISTH BAT score, and medication use were evaluated again. Blood was taken to repeat and extend laboratory tests for VWD (including multimer pattern, VWF-FVIII binding, VWFcollagen binding) and PFD (including ATP release and flow cytometry).

\section{Multiparameter thrombus formation using microfluidics}

Multiparameter microfluidic assays were performed using the Maastricht flow chamber, as described previously in detail29,33, but adapted to operation at physiological temperature of $37^{\circ} \mathrm{C}^{38}$. Brightfield and tri-color fluorescence images were taken with an EVOS-FL microscope (Life Technologies), every minute during an overall time of 7 minutes.

Platelet adhesion and aggregation were assessed using the following parameters (Table 4.1): platelet surface area coverage: obtained from threshold, representing identified regions of all adhered platelet and thrombus structure $(P 1)$; platelet deposition: identified regions of platelet deposition and mono- or multi-layered thrombi (P2); thrombus morphology score (P3); thrombus contraction score $(P 4)$ and thrombus multilayer score $(P 5)$. Fibrinogen binding was assessed to report on integrin $\alpha_{11 b} \beta_{3}$ activation (P6). Phosphatidylserine (PS) exposure (P7) was measured as a marker of platelet procoagulant activity. Platelet secretion was assessed by measuring P-selectin expression (P8).

Platelet adhesion and aggregation (P1-5) were assessed at a collagen I surface (M1) at a wall-shear rate of $1000 \mathrm{~s}^{-1}$ (S1). Fluorescence parameters (P6-8) were assessed at a collagen I and a collagen III surface (M2) at wall-shear rate of $1000 \mathrm{~s}^{-1}$ and $1600 \mathrm{~s}^{-1}$ (S2). Figure 4.1 shows representative images of the results of a healthy individual and a patient with severe (type 1) GT. 
Table 4.1 Overview of microspot surfaces $(M)$, microfluidic parameters $(P)$, and flow cytometry markers $(C)$.

\begin{tabular}{llll}
\hline Microspot surface & \multicolumn{2}{l}{ Platelet receptor involved } \\
\hline M1 & Collagen type I & GP1b, GPVI, $\alpha_{2} \beta_{1}$ & \\
M2 & Collagen type III & GP1b, GPVI, $\alpha_{2} \beta_{1}$ & \\
\hline Brightfield / Fluorescence parameters & Range & Normalized \\
\hline P1 & platelet surface area coverage (\%SAC) & $0-100$ & $0-10$ \\
P2 & platelet deposition (\%SAC) & $0-100$ & $0-10$ \\
P3 & thrombus morphological score & $0-6$ & $0-10$ \\
P4 & thrombus contraction score & $0-4$ & $0-10$ \\
P5 & thrombus multilayer score & $0-4$ & $0-10$ \\
P6 & Integrin $\alpha_{\text {IIb }} \beta_{3}$ activation (\%SAC) & $0-100$ & $0-10$ \\
P7 & PS exposure (\%SAC) & $0-100$ & $0-10$ \\
P8 & P-selectin expression (\%SAC) & $0-100$ & $0-10$ \\
\hline Flowcytometry markers & Platelet receptor involved \\
\hline C1 & CD41 & GPIIb (integrin $\left.\alpha_{\text {IIb }} \beta_{3}\right)$ & \\
C2 & CD42a & GPIX & \\
C3 & CD42b & GPIb (integrin $\left.\alpha_{2}\right)$ & \\
C4 & CD42d & GPV & \\
C5 & CD61 & GPIIla (integrin $\beta_{3}$ & \\
\hline
\end{tabular}

GP, glycoprotein; PS, phosphatidylserine; SAC, surface area coverage.

Figure 4.1 Microscopic imaging of platelet thrombus formation on two different microspots.

A

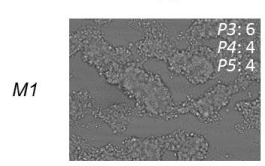

M2

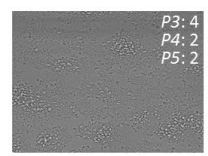

B

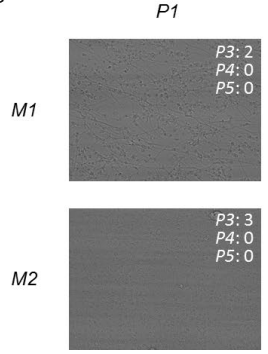

P2
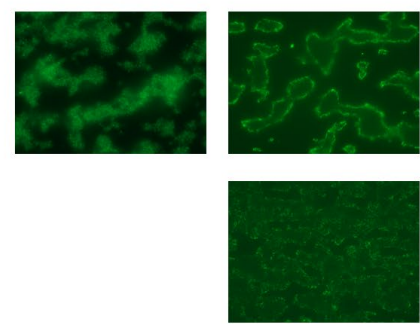

P6

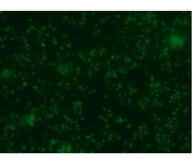

P6

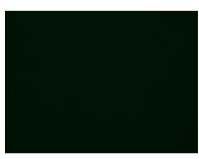

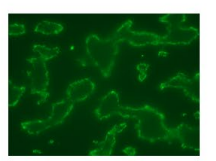
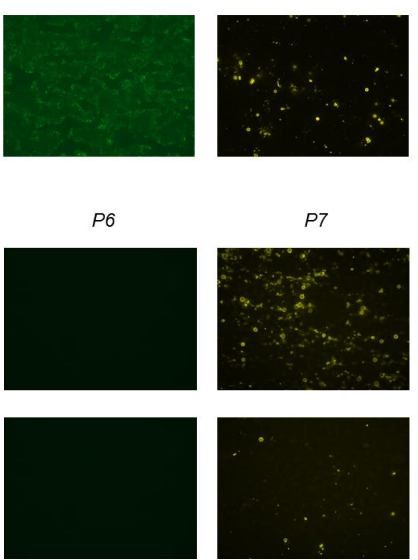

$P 7$

$P 7$
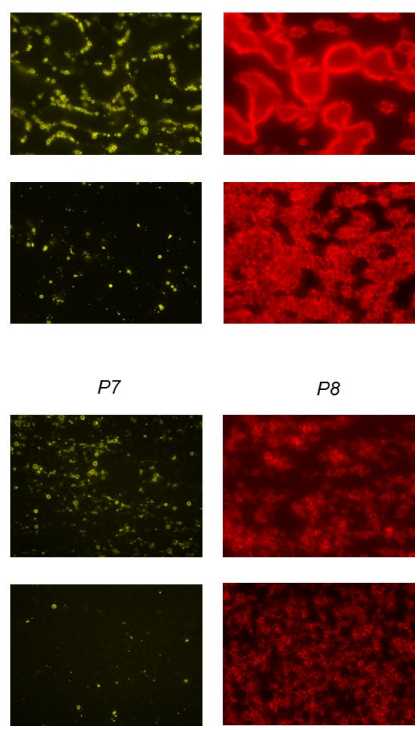

$P 8$

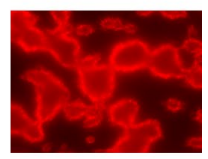

P8
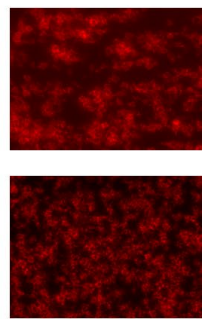

Representative images after whole blood flow from a healthy subject (A) and a patient with type 1 GT (B) over different microspots $(M 1, M 2)$ at shear rate $S 1$. For the brightfield images, score values for $P 3$ (thrombus morphological score), P4 (thrombus contraction score) and $P 5$ (thrombus multilayer score) at 7 minutes are indicated. The definition of all parameters is given in Table 4.1. 


\section{ThromboGenomics High-Throughput Sequencing (HTS)}

Patients were sequenced using the ThromboGenomics HTS panel test of diagnosticgrade genes known to harbor variants associated with rare bleeding, thrombotic, or platelet disorders. The ThromboGenomics HTS test sample preparation, sequencing protocols, tested genes, variant prioritization, interpretation and reporting, were extensively described before ${ }^{39,40}$.

\section{Results}

\section{Selection of patients and control subjects}

Laboratory data from a total of 425 patients with established or suspected bleeding disorders from three earlier observational studies ${ }^{37}$ were evaluated for the identification of 'PFA-only' patients. In the ProBe-AHP cohort (referred patients), $15 / 136(11 \%)$ of such patients were identified; in the PANE cohort (preoperative patients) this number was $2 / 240(1 \%)$, and in the BEPA cohort (patients with a bleeding disorder) it was 4/49 (8\%). From the 21 identified PFA-only patients, 14 consented to be included in this study (Figure 4.2).

From the BEPA cohort, two patients with VWD and two patients with GT were included as positive controls. For the microfluidic assay, blood samples from 50 healthy controls were evaluated, in order to obtain normal values for the sets of thrombus parameters. Brightfield microscopic images of shear-dependent thrombus formation at $37^{\circ} \mathrm{C}$ were recorded for all 50 subjects, while parallel fluorescence images were also recorded for 23 of them and in 15 of those 23 patients, PFA results were obtained as well.

\section{Baseline characteristics and alterations in PFA, platelet function and VWF in PFA-only patients}

Table 4.2 shows the prospective baseline characteristics of the 14 PFA-only patients and the subgroup of 23 controls with parallel fluorescent images. Baseline characteristics, retrospective and prospective data of PFA-only patients, controls and patients with a bleeding disorder are presented in Appendix 4.B Table S4.1-S4.4.

Compared to the controls, the PFA-CT results of the PFA-only patients were longer (mean PFA-CT ADP: $118 \pm 27$ vs. $94 \pm 13$ s, $p=0.007$; mean PFA-CT EPI: $150 \pm 42$ vs. $111 \pm 16 \mathrm{~s}, p=0.005$ ) and platelet volume was higher (mean MPV: $10.6 \pm 1.3 \mathrm{vs}$. $9.8 \pm 0.8 \mathrm{~nL}, p=0.016$ ). Furthermore, the patients were significantly older compared to the controls (mean age: $51 \pm 17$ vs. $34 \pm 10$ years, $p=0.003$ ). The majority of the PFA- 
only patients was female (86\%), had blood type O (64\%) and the mean ISTH BAT score was $10 \pm 4$.

Figure 4.2 Patient flow diagram.

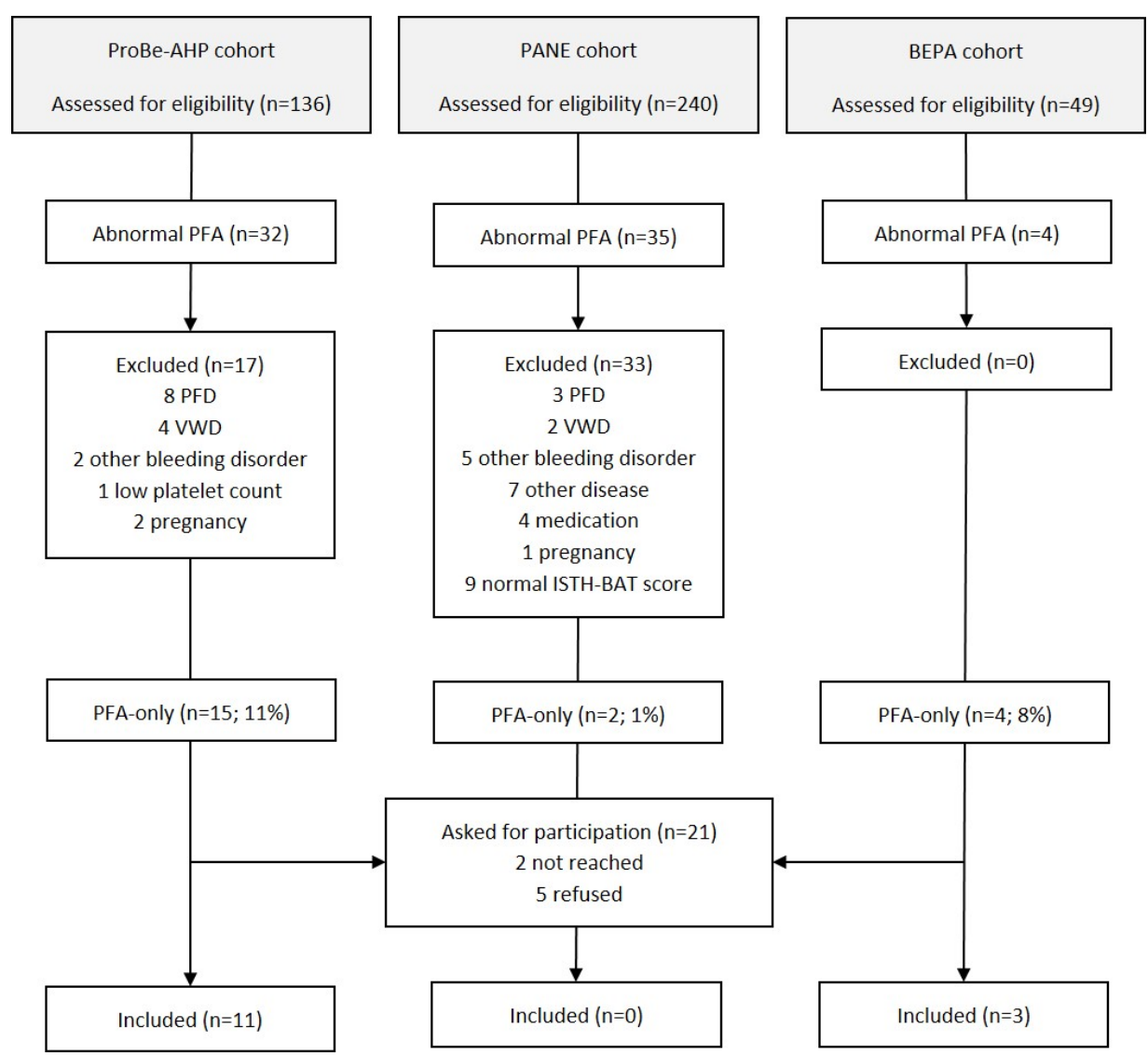

Flow diagram representing patient selection, reasons for exclusion and number of included patients from three observational studies, the ProBe-AHP, PANE and BEPA cohort. CT, closure time; ISTH BAT, international society on thrombosis and hemostasis-bleeding assessment tool; LTA, light transmission aggregometry; $n$, number; PFA, platelet function analyzer; PFD, platelet function disorder; VWD, von Willebrand disease; VWF, von Willebrand factor.

Extensive VWF tests, ATP release and flow cytometry were within the normal ranges in all 14 patients. Prospective LTA results showed two abnormal aggregation curves in one patient (nr. 8), fulfilling the diagnostic criteria of a platelet function disorder $21,22,41,42$. Patient nr. 9 used Venlafaxine, an SSRI, possibly explaining the prolonged PFA-CT. 
Table 4.2 Baseline characteristics of PFA-only patients $(n=14)$ and control group $(n=23)$.

\begin{tabular}{lccc}
\hline Characteristics & PFA-only patients & Controls & $p$ (95\%Cl) \\
mean \pm SD, $\mathbf{n}(\%)$ & $12(86)$ & $16(70)$ & 0.273 \\
\hline Female & $51 \pm 17$ & $34 \pm 10$ & $0.003(6.257-26.687)$ \\
Age (years) & $9(64)$ & & \\
Blood type O & $10 \pm 4$ & & \\
ISTH BAT score* & $0.42 \pm 0.03$ & $0.42 \pm 0.03$ & $0.848(-0.0221-0.018)$ \\
Hematocrit (L/L) & $257 \pm 84$ & $279 \pm 68$ & $0.374(-73.727-28.435)$ \\
Thrombocytes (x10\%/L) & $10.6 \pm 1.3$ & $9.8 \pm 0.8$ & $0.016(0.165-1.495)$ \\
MPV (nL) & $5.7 \pm 1.1$ & $6.2 \pm 1.6$ & $0.290(-1.479-0.455)$ \\
Leukocytes (x10\%/L) & $150 \pm 42$ & $111 \pm 16$ & $0.005(13.558-63.728)$ \\
PFA-EPI (s) & $118 \pm 27$ & $94 \pm 13$ & $0.007(7.453-41.204)$ \\
PFA-ADP (s) & $93 \pm 33$ & & \\
VWF activity (\%) & $91 \pm 32$ & & \\
VWF antigen (\%) & $1.0 \pm 0.1$ & & \\
VWF ratio & $125 \pm 39$ & & \\
FVIII (\%) & $0(0)$ & & \\
Multimer pattern abnormal & $82 \pm 31$ & & \\
VWF-Collagen binding (\%) & $94 \pm 12$ & & \\
VWF-FVIII binding (\%) & $0(0)$ & & \\
Flowcytometry abnormal & & & \\
\hline
\end{tabular}

*An ISTH BAT score for male $\geq 4$ and female $\geq 6$ was considered to be abnormal. ADP, adenosine diphosphate; $\mathrm{Cl}$, confidence interval; EPI, epinephrine; ISTH BAT, international society on thrombosis and hemostasis bleeding assessment tool; MPV, mean platelet volume; $n$, number; PFA, platelet function analyzer; s, seconds; SD, standard deviation; VWF, von Willebrand factor.

\section{Altered microfluidic thrombus formation in PFA-only patients}

Multiparameter microfluidic assessment of shear-dependent thrombus formation, operating shear rate of $1000 \mathrm{~s}^{-1}$ and $1600 \mathrm{~s}^{-1}$ and physiological temperature of $37^{\circ} \mathrm{C}$, was performed with blood from all patients. Results are shown in Figure 4.3 (data are presented in Appendix 4.B Table S4.5).

In the cohort of PFA-only patients, as compared to healthy volunteers, microfluidic test results showed significantly lower platelet surface coverage $(P 1)$ and platelet deposition $(P 2)$ at 3 and 7 minutes (P1_7min: 49 vs. 61\%, $p<0.001 ; P 2$ 7min: 17 vs. $22 \%, p<0.001)$ and significantly lower thrombus formation scores at all time points ( $p<0.008$ for all comparisons). Fluorescence markers showed lower integrin activation (P6: 20 vs. 27\%, $p=0.001$ ), PS exposure (P7: 12 vs. 16\%, $p=0.036$ ) and P-selectin expression ( $P 8$ : 33 vs. 41\%, $p=0.001$ ). No significant difference was seen in the results of PFA-only patients compared to the results of patients with a known bleeding disorder. Thrombus contraction and multilayer score $(P 4, P 5)$ were even less in PFAonly patients when compared to patients with a bleeding disorder, however this was not significant. 
Figure 4.3 Microfluidic results of PFA-only patients $(n=14)$, patients with a bleeding disorder $(n=4)$ and control groups $(n=50 / 23)$.

Platelet surface area coverage (\%SAC)

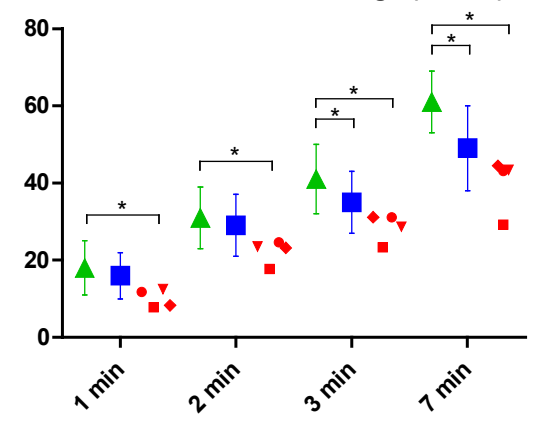

Thrombus morfological score (0-6)

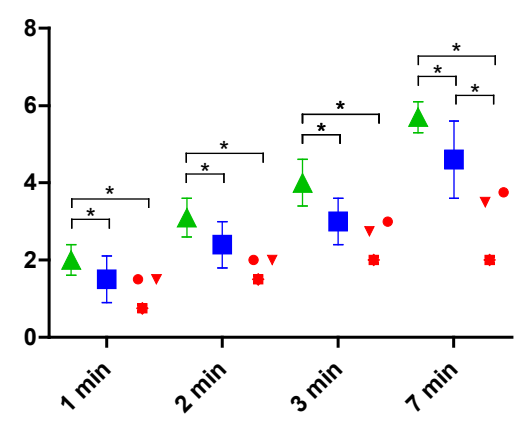

Thrombus multilayer score (0-4)

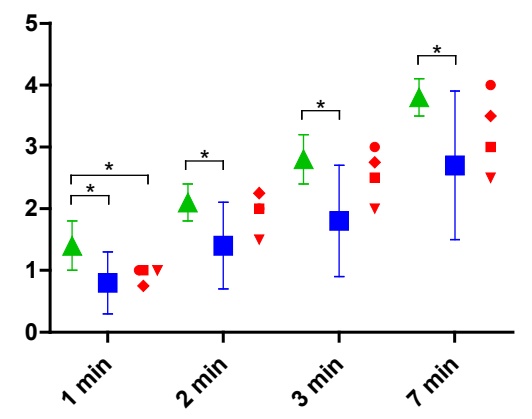

Platelet deposition (\%SAC)

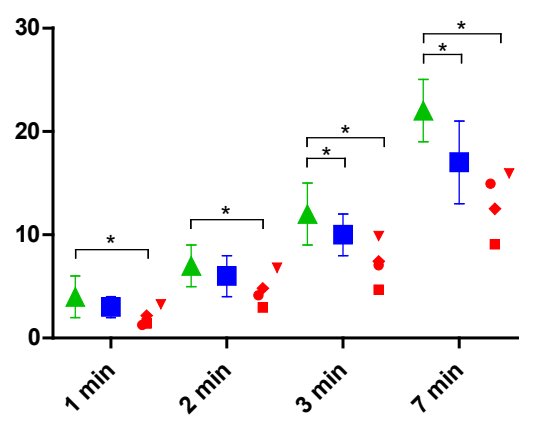

Thrombus contraction score (0-4)

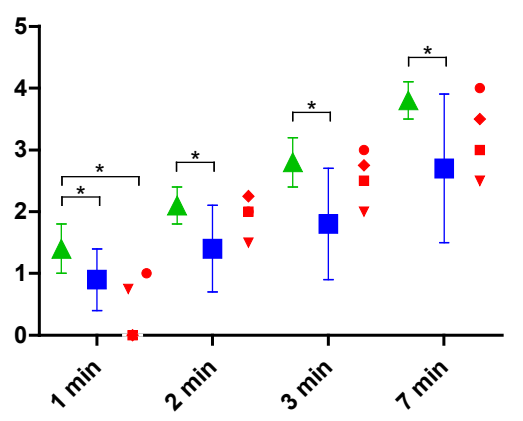

Integrin $\alpha$ llb $\beta 3$ activation (\%SAC)

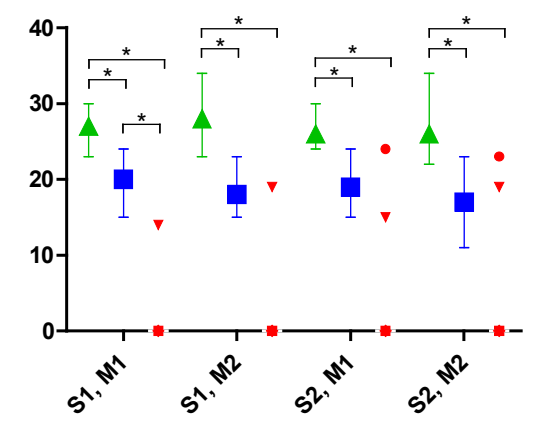



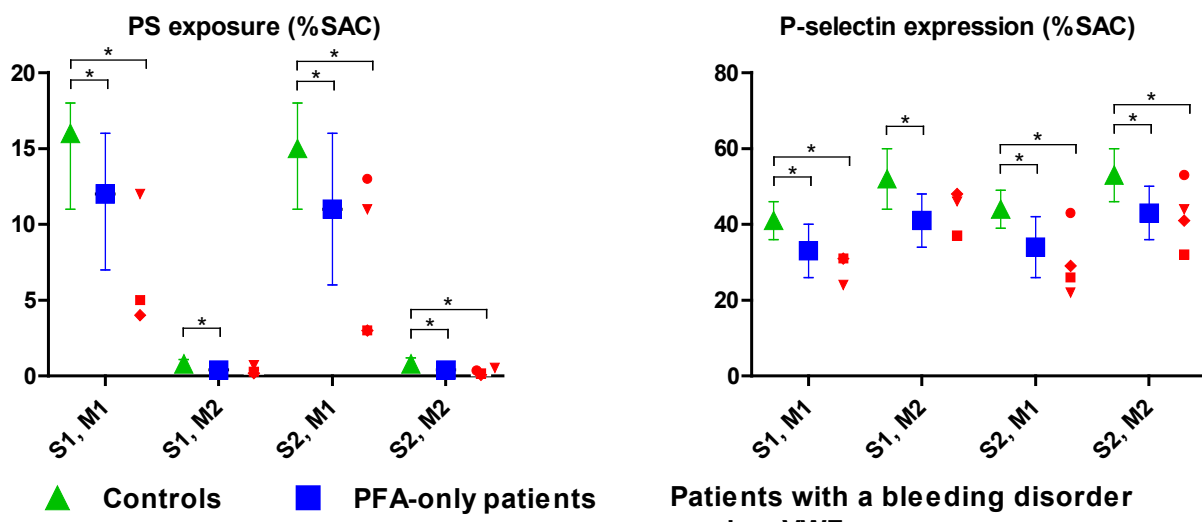

Patients with a bleeding disorder

- low VWF

$\checkmark$ VWD type 2A

- GT type 3

- GT type 1

Comparison of microfluidic result of all patients. Parameters $P 1-5$ at surface $M 1$ and shear rate $S 1$ are compared between 50 controls (green triangle), PFA-only patients (blue square), and patients with a bleeding disorder (in red, each symbol represents a patient). Parameters $P 6-8$ at surface $M 1$ and $M 2$ and shear rate $S 1$ and $S 2$ are compared between PFA-only patients, patients with a bleeding disorder and 23 controls. *alpha level of significance $=p<0.05$. Min, minutes; PFA, platelet function analyzer; SAC, surface area coverage. For detailed explanation of parameters $(P)$, microspots $(M)$ and shear rates $(S)$ see Table 4.1.

In Figure 4.4, the results of the microfluidic parameters are visualized per individual patient. In all patients abnormalities in microfluidic parameters were found, indicating reduced thrombus formation, compared to the group of controls. In PFAonly patients $8,9,12,13$ and 14 severe abnormalities in thrombus formation were found, comparable to patients with VWD or thrombopathy.

\section{Integration of data sets}

For a systemic evaluation of the characteristics, correlation analysis were performed for the laboratory results and microfluidic parameters in the 14 PFA-only patients. Integrated results of the correlations coefficients $R$ and $p$ values are visualized in Figure 4.5A and 4.5B, respectively (results of all subjects are presented in Appendix 4.C Figure $S 4.1 \mathrm{~A}$ and $\mathrm{B}$ ). The PFA-CT results correlated moderately with VWF activity $(\mathrm{R}=-0.58, p<0.031)$ and with PS exposure $(P 7)$ at $M 2, S 2(\mathrm{R}=-0,62, p=0.022)$ but not with other microfluidic parameters. Moderate to strong correlations were seen between microfluidic parameters and LTA aggregation curves, but not with ATP release. Furthermore, both LTA results and microfluidic parameters correlated with hematocrit ( $\mathrm{R}$ range $=0.40-0.77, p<0.05)$. 


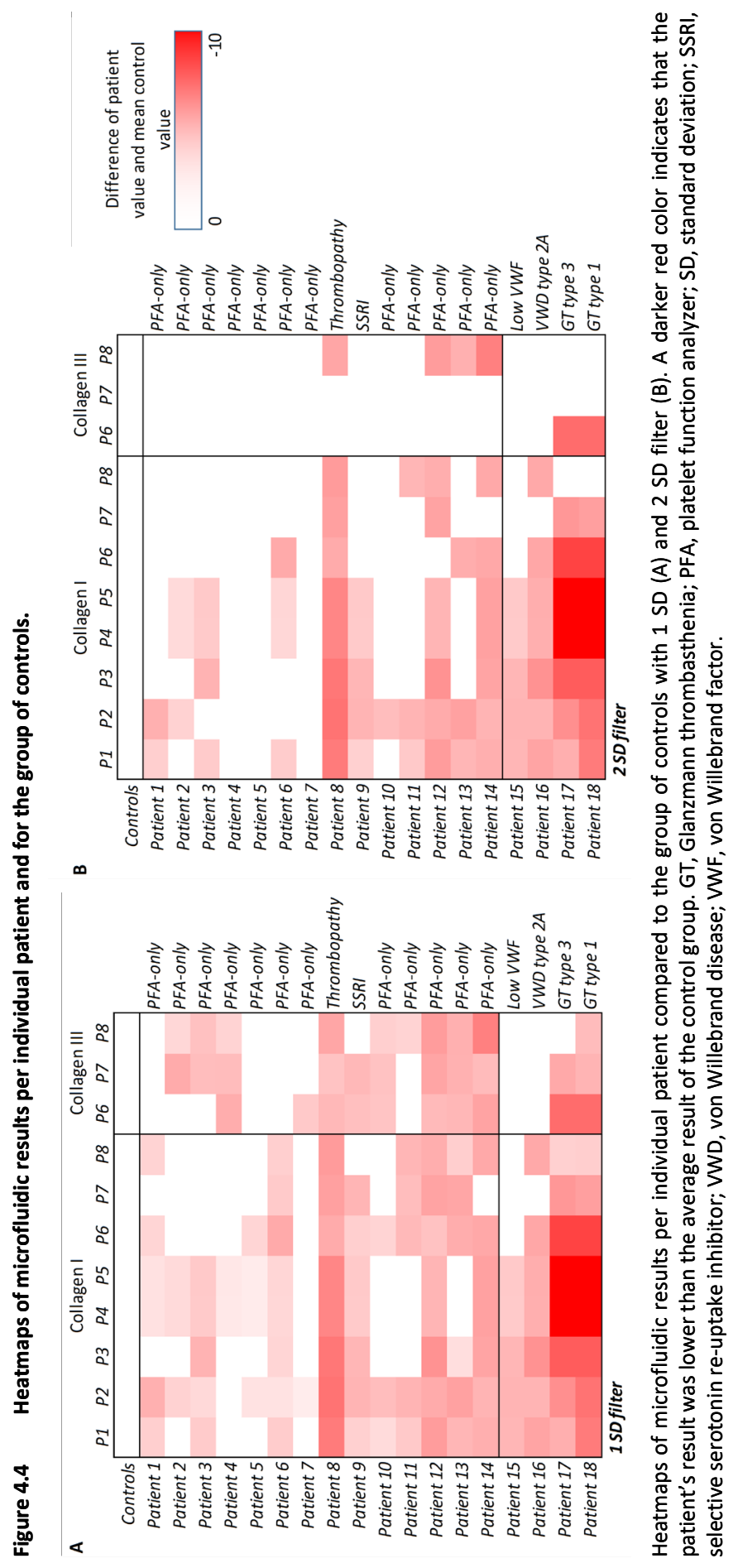


Principle component analysis (PCA) was performed to find the gross relationships between the microfluidic parameters at $S 1, M 1$ and the subject parameters in the PFA-only patients (Figures 4.6A and 4.6B). Markedly, Figure 4.6A shows the extent of clustering of microfluidic parameters $P 1-8$ and hematocrit in component 1 (42\%). Neither age, sex nor BAT contributed to this component. In component 2 (22\%) the other hematology parameters clustered together. Figure $4.6 \mathrm{~B}$ shows the extent of clustering of microfluidic parameters, LTA aggregation curves and hematocrit in component 1 (52\%), while PFA with VWF and to a lesser extent P6 clustered in component 2 (18\%).

To further investigate the parameters in the PFA-only patients, comparative analysis was performed in subgroups stratified for results below respectively above the mean value of all patients, for PFA (total of PFA-CT EPI and ADP), VWF activity, scaled microfluidic results, and hematocrit. Results are shown in Appendix 4.B Table S4.6. Patients with more prolonged PFA results (7/14), had less integrin activation ( $P 6$ : $17 \pm 3$ vs. $23 \pm 4 \%, p=0.011)$ in thrombus formation, and a trend towards a lower VWF activity (76 \pm 13 vs. $109 \pm 39 \%, p=0.070$ ), compared to patients with higher PFA results. Patients with lower VWF activity $(8 / 14)$ had significantly prolonged PFA-CT EPI compared to patients with higher VWF (PFA-CT EPI: $169 \pm 38$ vs. $124 \pm 33$ s., $p=0.038$ ), but no differences were found in microfluidic test results.

Patients with severe abnormal microfluidic results (5/14), had lower LTA aggregation curves (although not considered as diagnostically relevant) and less ATP release on epinephrine compared to patients with less severe abnormalities (e.g. LTA NAAA: $72 \pm 7$ vs. $85 \pm 4 \%, p=0.001$; LTA ADP5: $63 \pm 11$ vs. $81 \pm 4 \%, p=0.001$; ATPrEPI: $1.19 \pm 0.09$ vs. $1.54 \pm 0.41 \mathrm{nmol}, p=0.032$ ), there was no significant difference in VWF activity. Finally, patients with lower hematocrit (6/14) had lower LTA aggregation curves and more abnormal microfluidic test results (e.g. LTA NAAA: $74 \pm 8$ vs. $84 \pm 5 \%, p=0.014$; platelet deposition $P 2: 14 \pm 3$ vs. $20 \pm 2 \%, p=0.001)$.

\section{Results of high-throughput sequencing of PFA-only patients}

The ThromboGenomics HTS gene panel test results were available for all PFA-only patients. The patients were tested with the ThromboGenomics version 3 (TG.V3), including 96 Mendelian genes causing coagulation, thrombotic, or platelet disorders and probes for 10,000 common single-nucleotide variants (SNVs) to estimate relatedness and ancestry ${ }^{39}$. No genetic variants (SNV or structural variants) that could explain the coagulation or platelet abnormality were identified. 
Figure 4.5 Correlation analysis to reveal interactions between microfluidic parameters, hematology, PFA, platelet function tests, age, sex, and BAT score in PFA-only patients $(n=14)$.

A

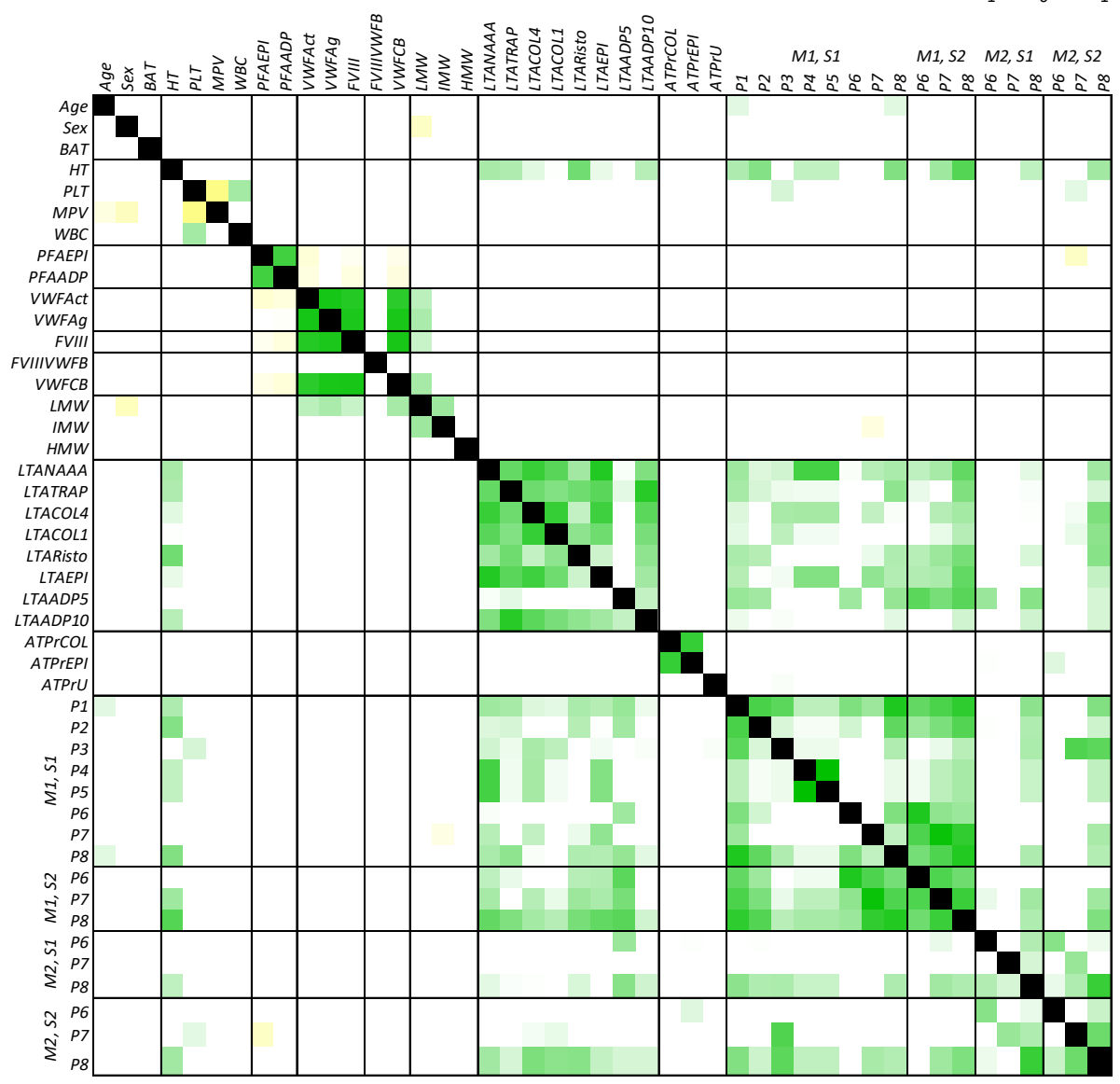

Heat mapped correlations (R) with $p$ values of microfluidic parameters and subject parameters in the 14 PFA-only patients. (A) Heat mapped Pearson correlation coefficients R: a darker color indicates a stronger negative (yellow) or positive (green) correlation. (B) Heat mapped $p$ values in which a darker color (blue) indicates a highly significant correlation (white offset at $p=0.05$ ). ADP, adenosine diphosphate; ATPr, adenosine triphosphate release; BAT, bleeding assessment tool score; COL, collagen; EPI, epinephrine; 


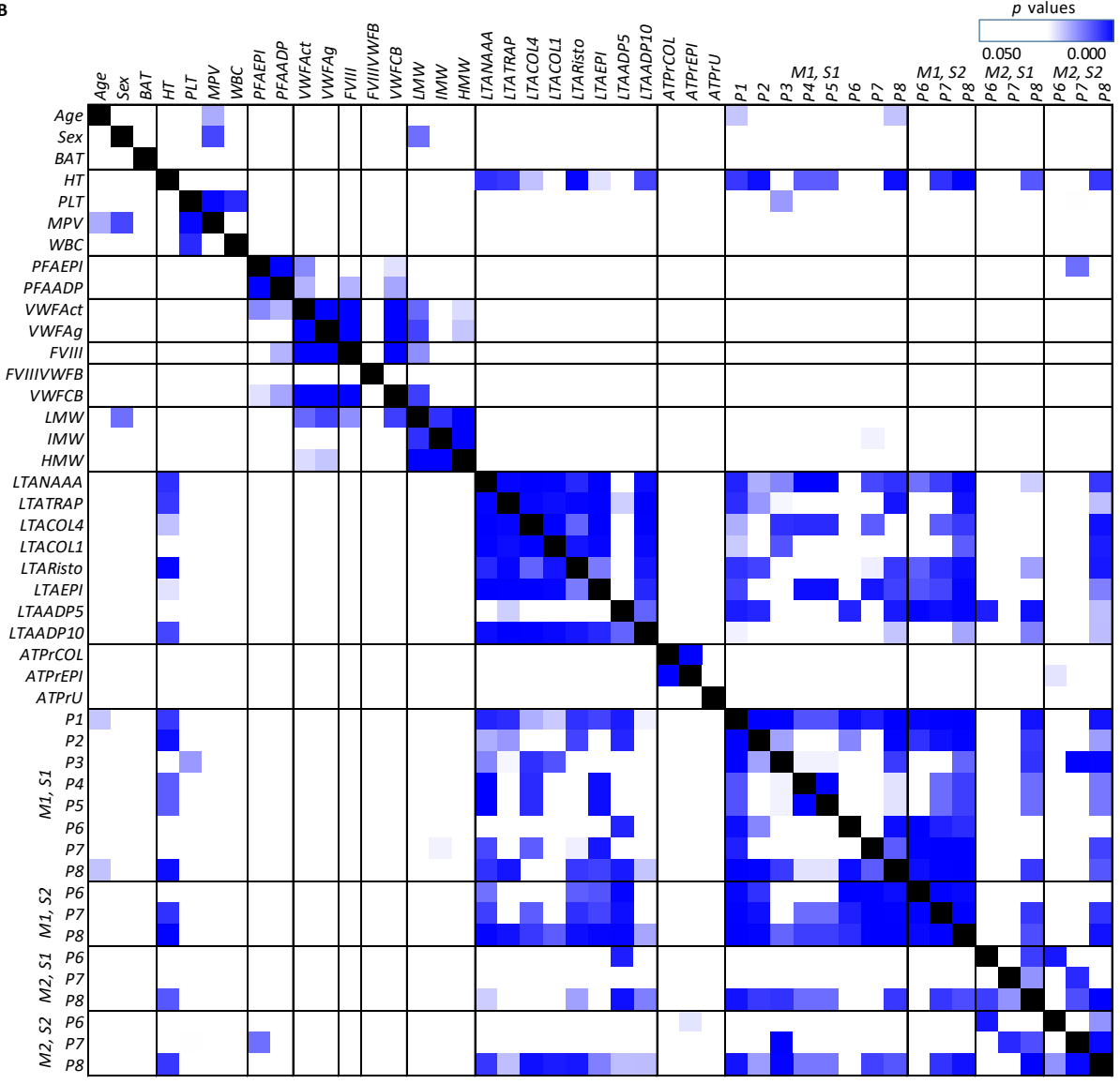

$\mathrm{HT}$, hematocrit; LTA, light transmission aggregometry; MPV, mean platelet volume; NAAA, arachidonic acid; PFA; platelet function analyzer; PLT, platelet count; $R$, correlation coefficient; RISTO, ristocetin; TRAP, thrombin receptor activatable peptide aggregometry; PFA, platelet function analyzer; $U$, thromboxane A2 analog; WBC, white blood cell count; VWFact, von Willebrand factor activity; VWFag, von Willebrand factor antigen. For detailed explanation of parameters $(P)$, microspots $(M)$ and shear rates $(S)$ see Table 4.1. 
Figure 4.6 Principal component analysis to reveal correlations between the microfluidic parameters and subject parameters in PFA-only patients $(n=14)$.

A

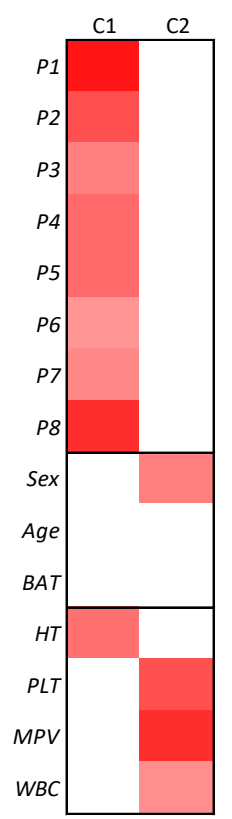

B

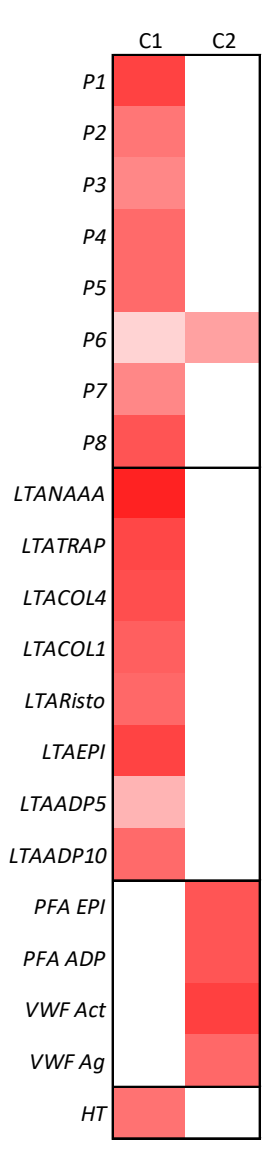

Relative contribution

to component

Principal component analysis of microfluidic parameters $P 1-8$ at surface $M 1$ at shear rate $S 1$ and $(A)$ sex, age, BAT score, HT, PLT, MPV and WBC score, (B) LTA aggregation curves, PFA, VWF and HT. Darker (red) colors indicate the parameters that tend to cluster together per component when compared to other sets of parameters. ADP, adenosine diphosphate; BAT, bleeding assessment tool score; COL, collagen; EPI, epinephrine; HT, hematocrit; LTA, light transmission aggregometry; MPV, mean platelet volume; NAAA, arachidonic acid; PFA; platelet function analyzer; PLT, platelet count; RISTO, ristocetin; TRAP, thrombin receptor activatable peptide aggregometry; $U$, thromboxane $A 2$ analogue; WBC, white blood cell count; VWFact, von Willebrand factor activity; VWFag, von Willebrand factor antigen. For detailed coding of parameters $(P)$, microspots $(M)$ and shear rates $(S)$ see Table 4.1 . 


\section{Discussion}

In this study we investigated whether and how the multiparameter shear-dependent microfluidic assay could help to identify abnormalities in platelets or VWF that can explain the prolonged closure times of PFA-only patients with a bleeding history.

Shear stress and shear rate dependent mechanisms play important roles in primary hemostasis. The static conventional platelet function tests do not evaluate thrombus formation under high wall shear. Whole blood microfluidic assays mimic the hemodynamic conditions of the vasculature and the complex cellular interactions with the vessel wall. In addition to the PFA test, the microfluidic test has multiple outcome parameters, enabling evaluation and analysis of 'real life' thrombus formation and platelet activation in greater detail. Furthermore, additional microspot surfaces can be used to give more detailed insight into altered platelet reactivity and receptor functions ${ }^{29}$.

We found that in PFA-only patients: $(i)$ most microfluidic test parameters were impaired compared to healthy controls. Abnormalities reached to levels seen in patients with established bleeding disorders, such as Glanzmann thrombasthenia and VWD; (ii) PCA analysis indicated VWF and platelet activation as co-variables of PFA results; (iii) LTA aggregation results and hematocrit were indicated as co-variables of microfluidic parameters; (iv) in patients with the most severe microfluidic abnormalities, LTA aggregation results were in the lowest range of normal; $(v)$ patients with lower hematocrit had more abnormal microfluidic test results; ( $v i$ ) exome sequencing did not reveal a monogenic explanation for the abnormalities found.

Our results show that an abnormal PFA as a single aberrant test in patients referred for bleeding evaluation, has to be considered for further evaluation. We found that PFA-only patients exhibited abnormal multiparameter microfluidic test results, and analysis of the specific microfluidic outcome parameters indicated impaired thrombus formation and less platelet activation in these patients. In some PFA-only patients abnormalities were even comparable to patients with previously established VWD or a PFD. PFA prolongation was associated with low-normal VWF, while microfluidic abnormalities were also associated with low normal LTA aggregation curves and low hematocrit.

Aberrations in microfluidic assays have been described in patients with mild to severe platelet function defects like Gray platelet syndrome, storage pool disease, Bernard-Soulier and Glanzmann thrombasthenia ${ }^{29,30}$, but also in patients with VWD type 1 and low $V_{W} F^{30,43}$. The correlations of microfluidic test results with LTA aggregation results, found in our study, indicate that the abnormalities found in these 
patients could reflect a shear dependent platelet activation defect, that is not detected using the current thresholds for normality in the static conventional platelet function tests.

We found no correlation between VWF levels, multimer pattern, VWF-collagen binding or VWF-FVIII binding and microfluidic test parameters. However, this does not exclude that defects in VWF, affecting its platelet binding function under high shear rate, could contribute to the abnormal microfluidic test results found in these patients.

Exome sequencing of a large panel of genes associated with coagulation, thrombotic, or platelet disorders, did not reveal a monogenic explanation for the abnormalities found in the PFA-only patients. Again, this does not exclude genetic variations in unknown genes involved in the VWF-platelet interactions, or the accumulative effects of a number of common variants ${ }^{39}$, which might result in the abnormalities found.

In the PFA-only patients, but not in all subjects, hematocrit correlated with the microfluidic test results. Patients with low normal hematocrit had more abnormal microfluidic test results. Hematocrit is known to influence platelet interaction with the vessel wall, but the effect is limited to levels below $0.25 \mathrm{~L} / \mathrm{L}^{44}$. A previous study of a microfluidic assay in healthy individuals, found no association between hematocrit and the microfluidic assay results ${ }^{45}$. However, in PFA-only patients, reduced red blood cell dependent platelet migration towards the vessel wall, might aggravate the abnormalities of platelet function found in the microfluidic assay.

Based on the present analysis, we propose that, in all patients with an unexplained abnormal PFA, microfluidic testing should be considered in the diagnostic work-up for mild bleeding tendency as an additional test of shear-dependent VWF or platelet function. Our results also indicate that it would be interesting to perform microfluidic testing in all patients with bleeding of unknown cause (BUC). Only one study investigated the results of a microfluidic assay in a group of ten BUC patients and found no significant differences compared to the healthy controls ${ }^{43}$. However, the microfluidic assay used in this study differed greatly from our assay.

This study is limited by the small number of PFA-only patients, of which 2 patients prospectively had an explanation for the prolongation of PFA-CT. This prevented us from performing a robust regression analysis and therefore, our results should be interpreted with care. However, we still believe that our findings add important information to the discussion about BUC patients with an unexplained abnormal PFA and give directions for further research.

In conclusion, our study showed that an abnormal PFA as a single aberrant test in bleeding patients should be further evaluated. The multiparameter microfluidic test is 
able to detect abnormalities in these patients, also detecting low normal LTA aggregation results, suggesting a shear-dependent platelet function defect, not detected by the static conventional platelet function tests. 


\section{References}

1. Kratzer MA, Born GV. Simulation of primary haemostasis in vitro. Haemostasis 1985;15(6):357-62.

2. Favaloro EJ. Clinical utility of closure times using the platelet function analyzer-100/200. Am J Hematol 2017;92(4):398-404.

3. Harrison P. The role of PFA-100 testing in the investigation and management of haemostatic defects in children and adults. BJH 2005;130(1):3-10.

4. Karger $R$, et al. Diagnostic performance of the platelet function analyzer (PFA-100 (R)) for the detection of disorders of primary haemostasis in patients with a bleeding history - a systematic review and meta-analysis. Platelets 2007;18(4):249-60.

5. Cattaneo M. Are the bleeding time and PFA-100R useful in the initial screening of patients with mucocutaneous bleedings of hereditary nature? J Thromb Haemost 2004;2(6):890-1.

6. Ardillon $L$, et al. Platelet function analyser (PFA-100) results and von Willebrand factor deficiency: a 16-year 'real-world' experience. Haemophilia 2015;21(5):646-52.

7. Favaloro EJ. Utility of the platelet function analyser (PFA-100/200) for exclusion or detection of von Willebrand disease: A study 22 years in the making. Thromb Res 2020;188:17-24.

8. Fressinaud $\mathrm{E}$, et al. Screening for von Willebrand disease with a new analyzer using high shear stress: A study of 60 cases. Blood 1998;91(4):1325-31.

9. Cattaneo $\mathrm{M}$, et al. Evaluation of the PFA-100 system in the diagnosis and therapeutic monitoring of patients with von Willebrand disease. Thromb Haemost 1999;82(1):35-9.

10. Favaloro EJ, Pasalic L, Curnow J. Monitoring Therapy during Treatment of von Willebrand Disease. Semin Thromb Hemost 2017;43(3):338-54.

11. van Vliet $\mathrm{HH}$, et al. PFA-100 monitoring of von Willebrand factor (VWF) responses to desmopressin (DDAVP) and factor VIII/VWF concentrate substitution in von Willebrand disease type 1 and 2 . Thromb Haemost 2008;100(3):462-8.

12. Cattaneo $\mathrm{M}$, et al. Evaluation of platelet function with the PFA-100 system in patients with congenital defects of platelet secretion. Thromb Res 1999;96(3):213-7.

13. Moenen FCJ, et al. Screening for platelet function disorders with Multiplate and platelet function analyzer. Platelets 2019;30(1):81-7.

14. Kerenyi A, et al. Comparison of PFA-100 closure time and template bleeding time of patients with inherited disorders causing defective platelet function. Thromb Res 1999;96(6):487-92.

15. Podda GM, et al. Usefulness of PFA-100 (R) testing in the diagnostic screening of patients with suspected abnormalities of hemostasis: comparison with the bleeding time. J Thromb Haemost 2007; 5(12):2393-8.

16. Norman J, et al. How should we test for nonsevere heritable platelet function disorders? Int J Lab Hematol 2014;36(3):326-33.

17. Hayward C, et al. Platelet function analyzer (PFA)-100 closure time in the evaluation of platelet disorders and platelet function. J Thromb Haemost 2006;4(2):312-9.

18. Harrison $\mathrm{P}$, et al. The PFA-100: a potential rapid screening tool for the assessment of platelet dysfunction. Clin Lab Haematol 2002;24(4):225-32.

19. Favaloro EJ. The utility of the PFA-100 in the identification of von Willebrand disease: a concise review. in Seminars in thrombosis and hemostasis. 2006. Copyright@ 2006 by Thieme Medical Publishers, Inc., 333 Seventh Avenue, New York, NY 10001, USA.

20. Favaloro E.J. Clinical utility of the PFA-100. Semin Thromb Hemost 2008;34(8):709-33.

21. Gresele P. Diagnosis of inherited platelet function disorders: guidance from the SSC of the ISTH. J Thromb Haemost 2015;13(2):314-22.

22. Dawood BB, et al. Evaluation of participants with suspected heritable platelet function disorders including recommendation and validation of a streamlined agonist panel. Blood 2012;120(25):5041-9.

23. Pai $M$, et al. Diagnostic usefulness of a lumi-aggregometer adenosine triphosphate release assay for the assessment of platelet function disorders. Am J Clin Pathol 2011;136(3):350-8.

24. Sharma R, Haberichter SL. New advances in the diagnosis of von Willebrand disease. Hematology Am Soc Hematol Educ Program 2019;2019(1):596-600. 
25. Quiroga T, et al. High prevalence of bleeders of unknown cause among patients with inherited mucocutaneous bleeding. A prospective study of 280 patients and 299 controls. Haematologica 2007; 92(3):357-65.

26. Posan E, et al. Comparison of PFA-100 testing and bleeding time for detecting platelet hypofunction and von Willebrand disease in clinical practice. Thromb Haemost 2003;90(3):483-90.

27. MacDonald S, et al. Characterization of a large cohort of patients with unclassified bleeding disorder; clinical features, management of haemostatic challenges and use of global haemostatic assessment with proposed recommendations for diagnosis and treatment. Int J Lab Hematol 2019;42(2):116-25.

28. Obaji S, et al. Unclassified bleeding disorders: outcome of haemostatic challenges following tranexamic acid and/or desmopressin. Haemophilia 2016;22(2):285-91.

29. de Witt SM, et al. Identification of platelet function defects by multi-parameter assessment of thrombus formation. Nat Commun 2014;5:4257.

30. Brouns SLN, van Geffen JP, Heemskerk JWM. High-throughput measurement of human platelet aggregation under flow: application in hemostasis and beyond. Platelets 2018;29(7):662-9.

31. Petersen $\mathrm{R}$, et al. Platelet function is modified by common sequence variation in megakaryocyte super enhancers. Nat Commun 2017;8:16058.

32. Nagy $M$, et al. Variable impairment of platelet functions in patients with severe, genetically linked immune deficiencies. Haematologica 2018;103(3):540-9.

33. van Geffen JP, et al. High-throughput elucidation of thrombus formation reveals sources of platelet function variability. Haematologica 2019;104(6):1256-67.

34. Roest $\mathrm{M}$, et al. Flow chamber-based assays to measure thrombus formation in vitro: requirements for standardization. J Thromb Haemost 2011;9(11):2322-4.

35. Van Kruchten R, Cosemans JM, Heemskerk JW. Measurement of whole blood thrombus formation using parallel-plate flow chambers-a practical guide. Platelets 2012;23(3):229-42.

36. Heemskerk JW, et al. Collagen surfaces to measure thrombus formation under flow: possibilities for standardization. J Thromb Haemost 2011;9(4):856-8.

37. Moenen $\mathrm{F}$, et al. The diagnostic accuracy of bleeding assessment tools for the identification of patients with mild bleeding disorders: A systematic review. Haemophilia 2018;24(4):525-35.

38. Herfs L, et al. Multiparameter Microfluidics Assay of Thrombus Formation Reveals Increased Sensitivity to Contraction and Antiplatelet Agents at Physiological Temperature. Thromb Res 2021; 203:46-56..

39. Downes K, et al. Diagnostic high-throughput sequencing of 2396 patients with bleeding, thrombotic, and platelet disorders. Blood 2019;134(23):2082-91.

40. Megy $\mathrm{K}$, et al. Curated disease-causing genes for bleeding, thrombotic, and platelet disorders: Communication from the SSC of the ISTH. J Thromb Haemost 2019;17(8):1253-60.

41. Hayward CP. Diagnostic evaluation of platelet function disorders. Blood Rev 2011;25(4):169-73.

42. Cattaneo $\mathrm{M}$, et al. Recommendations for the Standardization of Light Transmission Aggregometry: A Consensus of the Working Party from the Platelet Physiology Subcommittee of SSC/ISTH. J Thromb Haemost 2013.

43. Lehmann $M$, et al. Evaluation of a microfluidic flow assay to screen for von Willebrand disease and low von Willebrand factor levels. J Thromb Haemost 2018;16(1):104-15.

44. Weisel JW, Litvinov RI. Red blood cells: the forgotten player in hemostasis and thrombosis. J Thromb Haemost 2019;17(2):271-82.

45. Neeves KB, et al. Sources of variability in platelet accumulation on type 1 fibrillar collagen in microfluidic flow assays. PLoS One 2013;8(1):e54680.

46. Linden MD, et al. Application of flow cytometry to platelet disorders. Semin Thromb Hemost 2004; 30(5):501-11.

47. Schmitz G, et al. European Working Group on Clinical Cell Analysis: Consensus protocol for the flow cytometric characterisation of platelet function. Thromb Haemost 1998;79(5):885-96. 


\section{Appendix 4.A Supplemental methods}

\section{Study population exclusion criteria}

Patients were excluded if they had normal PFA results; no further diagnostic information was available; a diagnosis of VWD (VWF activity or antigen $<50 \%$ ); a diagnosis of a platelet function disorder (low LTA with $\geq 2$ agonists, low ristocetin agglutination, or low ATP-release); a diagnosis of other bleeding disorders (e.g., coagulation factor deficiency); a low platelet count (<100 × 10\%/L); low hematocrit $(<0.25 \mathrm{~L} / \mathrm{L})$; interfering medication (e.g. antiplatelet or non-steroidal antiinflammatory drugs); or a medical history explaining a prolonged PFA-CT (for instance uremia, liver cirrhosis, hematologic disorders); were pregnant or lactating.

\section{Blood collection and work-up}

Patients were instructed to stop any interfering medication (e.g. aspirin, non-steroidal anti-inflammatory drugs) for 7 days before blood withdrawal. Patients were also asked to avoid taking fat-containing food for 4 hours. Blood was drawn from the antecubital vein by puncture using a $21 \mathrm{G}$ needle and collected in $3.2 \%$ sodium citrate Vacuette tubes ( $9 \mathrm{~mL}$; Greiner Bio-One, Austria). Measurements of PFA were performed within $1 \mathrm{~h}$ after blood withdrawal. Measurements of LTA (with ATP release) and microfluidics thrombus formation were performed within 3-4 h. For the preparation of platelet-rich plasma (PRP), citrated-blood was centrifuged at $170 \mathrm{~g}$ for $10 \mathrm{~min}$ at $18^{\circ} \mathrm{C}$. For the preparation of platelet free plasma (PFP), the citrated-blood was first centrifuged at $2500 \mathrm{~g}$ for $5 \mathrm{~min}$ at $18^{\circ} \mathrm{C}$. The plasma was then transferred to another tube, and re-centrifuged at $10000 \mathrm{~g}$ for $10 \mathrm{~min}$ at $18^{\circ} \mathrm{C}$.

\section{Diagnostic laboratory measurements}

Hemoglobin ( $\mathrm{Hb}$ ), platelet count and white blood cell (WBC) measurements were performed with a Sysmex XN 9000 analyzer (Sysmex Corporation, Kobe, Japan). Blood smears were evaluated for assessment of platelet size and morphology (AX10, Zeiss, Jena, Germany). Levels of factor VIII (actin FS, Siemens), were measured with a clotting assay using factor-depleted plasma on a Sysmex CS 2100i (Sysmex Corporation, Kobe, Japan), prothrombin times were assessed using Innovin reagent (Siemens, Marburg). Levels of VWF antigen and activity were determined on a Sysmex CS2100i analyser (VWF Reag and VWF Ac Reagens, Siemens). Multimers of VWF were assessed on a Hydrasys 2 instrument (Sebia, Evry Cedex. Collagen binding was assessed using a Technozym ${ }^{\circledR}$ VWF:CBA Eliza (Technoclone, Vienna) and factor VIII binding was performed with an assay from ASSERACHROM ${ }^{\circledR}$ VWF:FVIIIB assay (Diagnostica Stago S.A.S., Asnières sur Seine). 
For assessment of LTA and ATP release, a Chronolog aggregometer was used (Chrono-log Corporation, Havertown, USA). Platelet aggregation in PRP was induced with 5 or $10 \mu \mathrm{M}$ ADP (Chronolog CH384), 1 or $4 \mu \mathrm{g} / \mathrm{mL}$ collagen-I (Chrono-par Ref385), $10 \mu \mathrm{M}$ epinephrine (Chronolog CH393), $1 \mathrm{mM}$ arachidonic acid (Bio/Data) $1.5 \mathrm{mg} / \mathrm{mL}$ ristocetin (Chronolog-Stago Ref396), or $15 \mu \mathrm{M}$ thrombin receptor activating peptide (TRAP-6; Boom H8105). For ATP release measurements, platelets were activated by 5 $\mu \mathrm{g} / \mathrm{mL}$ collagen, $5 \mu \mathrm{M}$ epinephrine, or $1 \mu \mathrm{M}$ thromboxane $A_{2}$ analog U46619. Reference values for ATP release were $1.20-2.79 \mathrm{nmol}$ for collagen, $0.86-2.47 \mathrm{nmol}$ for epinephrine, and 0.39-1.87 nmol for U46619.

The platelet function analyzer 200 (PFA-200; Innovance PFA-200 System, Siemens, Marburg, Germany), was used to record closure times with the collagen/epinephrine (C-EPI) and the collagen/ADP (C-ADP) cartridges. The reference PFA-CT values were established as 84-160 s. for C-EPI and 68-118 s. for C-ADP. These values, provided by the manufacturer, were verified in our laboratory using blood samples from 23 healthy volunteers. Local within-run coefficient of variation for both PFA kits was $<10 \%$.

\section{Flow cytometry}

Platelet immunophenotyping was performed by a validated procedure based on previous papers ${ }^{1,2}$, using antibodies at recommended concentrations for the markers: CD41 (integrin $\alpha_{11 b} \beta_{3}$ complex, anti-CD41-PE Cy7, 303718, Biolegend), CD42a (GPIX, anti-CD42a-FITC, 348083, BD Biosciences), CD42b (GPIba, anti-CD42b-PE, 303906, Biolegend), CD42d (GPV, anti-CD42d-APC, FAB4249A, RnD Systems), CD61 (integrin $\beta_{3}$, anti-CD61-BV510, BD Biosciences). An overview is given in Table 4.1.

\section{Multiparameter thrombus formation using microfluidics}

Glass coverslips were coated with microspots of $2 \mu \mathrm{L}$ collagen I $(100 \mu \mathrm{g} / \mathrm{ml})$ and collagen III $(100 \mu \mathrm{g} / \mathrm{ml})$. Multiparameter microfluidic assays were performed using the Maastricht flow chamber, as described previously in detail ${ }^{3,4}$. An important modification was that whole blood flow was performed at a preset temperature of $37^{\circ} \mathrm{C}$, using an adapted temperature-controlled chamber holder, at wall-shear rates of $1000 \mathrm{~s}^{-1}$ or $1600 \mathrm{~s}^{-1}$. Prior to perfusion, citrated blood samples were recalcified in the presence of $40 \mu \mathrm{M}$ PPACK. Time kinetics were assessed by pre-staining with the membrane label $\mathrm{DiOC}_{6}(0.5 \mu \mathrm{g} / \mathrm{mL})$, after which the blood was flowed during an overall time of $7 \mathrm{~min}$, i.e. with recalcified blood for $3.5 \mathrm{~min}$., followed by rinse buffer (Hepes buffer pH $7.45+136 \mathrm{mM} \mathrm{NaCl}, 2.7 \mathrm{mM} \mathrm{KCl}, 2 \mathrm{mM} \mathrm{MgCl} 2,2 \mathrm{mM} \mathrm{CaCl}$, $1 \mathrm{mg} / \mathrm{mL}$ glucose, $1 \mathrm{mg} / \mathrm{mL}$ BSA and $1 \mathrm{U} / \mathrm{mL}$ heparin (Sigma-Aldrich)). For end stage measurements, whole blood perfusion was for 3.5 minutes with post-staining for 
FITC-labelled anti-fibrinogen mAb (1:100, Dako, F0111, Santa Clara, CA, USA), AF568 annexin A5 (1:200, Molecular Probes), and AF647 anti-CD62P monoclonal antibody $\left(1: 80\right.$, Biolegend, London, UK) ${ }^{4}$.

Brightfield and tri-color fluorescence images were taken with an EVOS-FL microscope (Life Technologies) equipped with GFP, RFP and Cy5 dichroic cubes and an Olympus UPLSAPO 60x oil-immersion objective. Brightfield and fluorescence images were taken, either every minute or after whole blood perfusion, to visualize and assess the parameters described in Table 4.1. Semi-automated scripts written in Fiji were used. Test variability was $7-20 \%{ }^{4}$, depending on the type of surface and parameter. Platelet adhesion and aggregation were assessed basically as before ${ }^{4}$.

\section{Statistical analyses}

Continuous variables were presented as either means \pm SD for normally distributed traits, or otherwise as medians \pm interquartile ranges. Categorical variables were expressed as counts and percentages. Differences between samples of PFA-only patients, patients with a known bleeding disorder and controls were tested with an unpaired t-test in case of normal distribution, with the Mann-Whitney $U$ test in case of not normal distribution and with a Fisher's exact test in case of categorical variables. Differences between subgroups within the PFA only patients were tested with an unpaired t-test in case of normal distribution, and the Wilcoxon matched pair signed rank test in case of not normal distribution.

Correlations were tested by Pearson's correlation or Spearman rank order correlation in SPSS. Correlation coefficients $\mathrm{R}$ and $p$ values were color coded and visualized in heatmaps. Statistical significance was assumed at $p$ values $<0.05$. Principal Component Analyses was performed in SPSS, using Oblimin rotation with Kaiser normalization. Results were color-coded and heat mapped.

Microfluidic results of patients were compared with results of a control group. Results were converted into heatmaps. Mean values of the defined outcome parameters were linearly scaled to a range from $0-10^{4}$. The individual patient values were subtracted from the mean control value, and visualized in a color-coded heatmap, which was filtered for mean \pm 1 SD and 2 SD. Color keys are shown next to the heatmaps as a reference. Principle component analysis (PCA) was performed to find the gross relationships between the microfluidic parameters and the subject parameters. Heat mapping of the relative contributions of components 1 and 2 indicated the parameters clustering together per component (in dark color), when compared to other sets of parameters.

Data were missing in case of a partially failed blood withdrawal, or when no blood was left to repeat the laboratory assay when it initially failed. Statistical analyses were 
performed with IBM SPSS statistics version 24.0. Graphs were made in GraphPad Prism version 5.0.

\section{References}

1. Linden MD, Frelinger AL, 3rd, Barnard MR, Przyklenk K, Furman MI, Michelson AD. Application of flow cytometry to platelet disorders. Semin Thromb Hemost 2004;30(5):501-11.

2. Schmitz G, Rothe G, Ruf A, Barlage S, Tschope D, Clemetson KJ, et al. European Working Group on Clinical Cell Analysis: Consensus protocol for the flow cytometric characterisation of platelet function. ThrombHaemost 1998;79(5):885-96.

3. de Witt SM, Swieringa F, Cavill R, Lamers MM, van Kruchten R, Mastenbroek T, et al. Identification of platelet function defects by multi-parameter assessment of thrombus formation. Nat Commun 2014;5:4257.

4. van Geffen JP, Brouns SLN, Batista J, McKinney H, Kempster C, Nagy M, et al. High-throughput elucidation of thrombus formation reveals sources of platelet function variability. Haematologica 2019;104(6):1256-67. 


\section{Appendix 4.B Supplemental tables}

Table S4.1 Retrospective and prospective baseline characteristics of PFA-only patients ( $n=14)$.

\begin{tabular}{lccc}
\hline Characteristics & Retrospective & Prospective & $\boldsymbol{p}$ (95\%Cl) \\
mean \pm SD, $\mathbf{n}(\%)$ & $12(86)$ & $12(86)$ & \\
\hline Female & $49 \pm 17$ & $51 \pm 17$ & \\
Age (years) & $9(64)$ & $9(64)$ & $0.349(0.525-1.382)$ \\
Blood type O & $10 \pm 4$ & $10 \pm 4$ & $0.166(-0.026-0.005)$ \\
ISTH BAT score & $0.40 \pm 0.03$ & $0.42 \pm 0.03$ & $0.130(-5.259-36.687)$ \\
Hematocrit (L/L) & $272 \pm 84$ & $257 \pm 84$ & $0.033(0.020-0.395)$ \\
Thrombocytes (x10\%/L) & $10.4 \pm 1.1$ & $10.6 \pm 1.3$ & $0.406(-0.203-0.470)$ \\
MPV (nL) & $5.8 \pm 1.3$ & $5.7 \pm 1.1$ & $0.090(-4.487-54.915)$ \\
Leukocytes (x10\%/L) & $175 \pm 42$ & $150 \pm 42$ & $0.032(1.384-26.045)$ \\
PFA-EPI (s) & $132 \pm 30$ & $118 \pm 27$ & $0.106(-21.649-2.363)$ \\
PFA-ADP (s) & $83 \pm 22$ & $93 \pm 33$ & $0.581(-13.774-8.060)$ \\
VWF activity (\%) & $88 \pm 21$ & $91 \pm 32$ & $0.301(-2.493-0.836)$ \\
VWF antigen (\%) & $0.9 \pm 0.1$ & $1.0 \pm 0.1$ & $0.218(-19.066-4.780)$ \\
VWF ratio & $118 \pm 26$ & $125 \pm 38$ & \\
FVIII (\%) & & $0(0)$ & \\
Multimer pattern abnormal (\%) & & $82 \pm 31$ & \\
VWF-Collagen binding (\%) & & $94 \pm 12$ & \\
VWF-FVIII binding (\%) & & $0(0)$ & \\
Flowcytometry abnormal (\%) & & & \\
\hline
\end{tabular}

$A D P$, adenosine diphosphate; $\mathrm{Cl}$, confidence interval; $\mathrm{EPI}$, epinephrine; ISTH BAT, international society on thrombosis and hemostasis-bleeding assessment tool; MPV, mean platelet volume; $n$, number; PFA, platelet function analyzer; SD, standard deviation; VWF, von Willebrand factor.

Table S4.2 Baseline results of control (sub)groups.

\begin{tabular}{lccc}
\hline $\begin{array}{l}\text { Characteristics } \\
\text { mean } \pm \text { SD, } \mathbf{n} \text { (\%) }\end{array}$ & $\begin{array}{c}\mathbf{5 0} \text { controls } \\
\text { (PFA } \mathbf{n = 1 8 )}\end{array}$ & $\begin{array}{c}\text { 23 controls } \\
\text { (PFA } \mathbf{n = 1 5 )}\end{array}$ & $\boldsymbol{p ~ ( 9 5 \% C l ) ~}$ \\
\hline Female & $31(62)$ & $16(70)$ & 0.606 \\
Age (years) & $35 \pm 12$ & $34 \pm 10$ & $0.846(-5.281-6.429)$ \\
Hematocrit (L/L) & $0.41 \pm 0.04$ & $0.42 \pm 0.03$ & $0.188(-0.029-0.006)$ \\
Thrombocytes(x10^9/L) & $269 \pm 64$ & $279 \pm 68$ & $0.532(-43.091-22.452)$ \\
MPV (nL) & $9.7 \pm 0.8$ & $9.8 \pm 0.8$ & $0.765(-0.464-0.342)$ \\
Leukocytes (x10^9/L) & $6.0 \pm 1.6$ & $6.2 \pm 1.6$ & $0.630(-1.001-0.615)$ \\
PFA-EPI (s) & $114 \pm 19$ & $111 \pm 16$ & $0.558(-9.097-16.541)$ \\
PFA-ADP (s) & $100 \pm 27$ & $94 \pm 13$ & $0.393(-8.882-22.015)$ \\
\hline
\end{tabular}

$A D P$, adenosine diphosphate; $\mathrm{Cl}$, confidence interval; $\mathrm{EPI}$, epinephrine; MPV, mean platelet volume; $n$, number; PFA, platelet function analyzer; s, seconds; SD, standard deviation. 
Table S4.3 Baseline characteristics of PFA-only patients and control groups.

\begin{tabular}{|c|c|c|c|c|c|}
\hline $\begin{array}{l}\text { Characteristics } \\
\text { mean } \pm \text { SD, n (\%) }\end{array}$ & $\begin{array}{c}\text { PFA-only } \\
\text { patients } \\
(n=14)\end{array}$ & $\begin{array}{c}50 \\
\text { controls }\end{array}$ & $\begin{array}{c}23 \\
\text { controls }\end{array}$ & $\begin{array}{c}p(95 \% \mathrm{Cl}) \\
\text { PFA-only vs. } 50 \text { controls }\end{array}$ & $\begin{array}{c}p(95 \% \mathrm{Cl}) \\
\text { PFA-only vs. } 23 \\
\text { controls } \\
\end{array}$ \\
\hline Female & $12(86)$ & $31(62)$ & $16(70)$ & 0.097 & 0.273 \\
\hline Age (years) & $51 \pm 17$ & $35 \pm 12$ & $34 \pm 10$ & $0.004(5.908-25.888)$ & $0.003(6.257-26.687)$ \\
\hline Hematocrit (L/L) & $0.42 \pm 0.03$ & $0.41 \pm 0.04$ & $0.42 \pm 0.03$ & $0.369(-0.012-0.031)$ & $0.848(-0.0221-0.018)$ \\
\hline Thrombocytes ( $\left.\times 10^{9} / \mathrm{L}\right)$ & $257 \pm 84$ & $269 \pm 64$ & $279 \pm 68$ & $0.556(-53.956-29.303)$ & $0.374(-73.727-28.435)$ \\
\hline $\operatorname{MPV}(\mathrm{nL})$ & $10.6 \pm 1.3$ & $9.7 \pm 0.8$ & $9.8 \pm 0.8$ & $0.002(0.330-1.452)$ & $0.016(0.165-1.495)$ \\
\hline Leukocytes (x109/L) & $5.7 \pm 1.1$ & $6.0 \pm 1.6$ & $6.2 \pm 1.6$ & $0.501(-1.245-0.616)$ & $0.290(-1.479-0.455)$ \\
\hline PFA-EPI (s) & $150 \pm 42$ & $114 \pm 19$ & $111 \pm 16$ & $0.010(9.688-60.153)$ & $0.005(13.558-63.728)$ \\
\hline PFA-ADP (s) & $118 \pm 27$ & $100 \pm 27$ & $94 \pm 13$ & $0.075(-1.900-37.424)$ & $0.007(7.453-41.204)$ \\
\hline
\end{tabular}

$A D P$, adenosine diphosphate; $\mathrm{Cl}$, confidence interval; $\mathrm{EPI}$, epinephrine; MPV, mean platelet volume; $n$, number; PFA, platelet function analyzer; s, seconds; SD, standard deviation. 


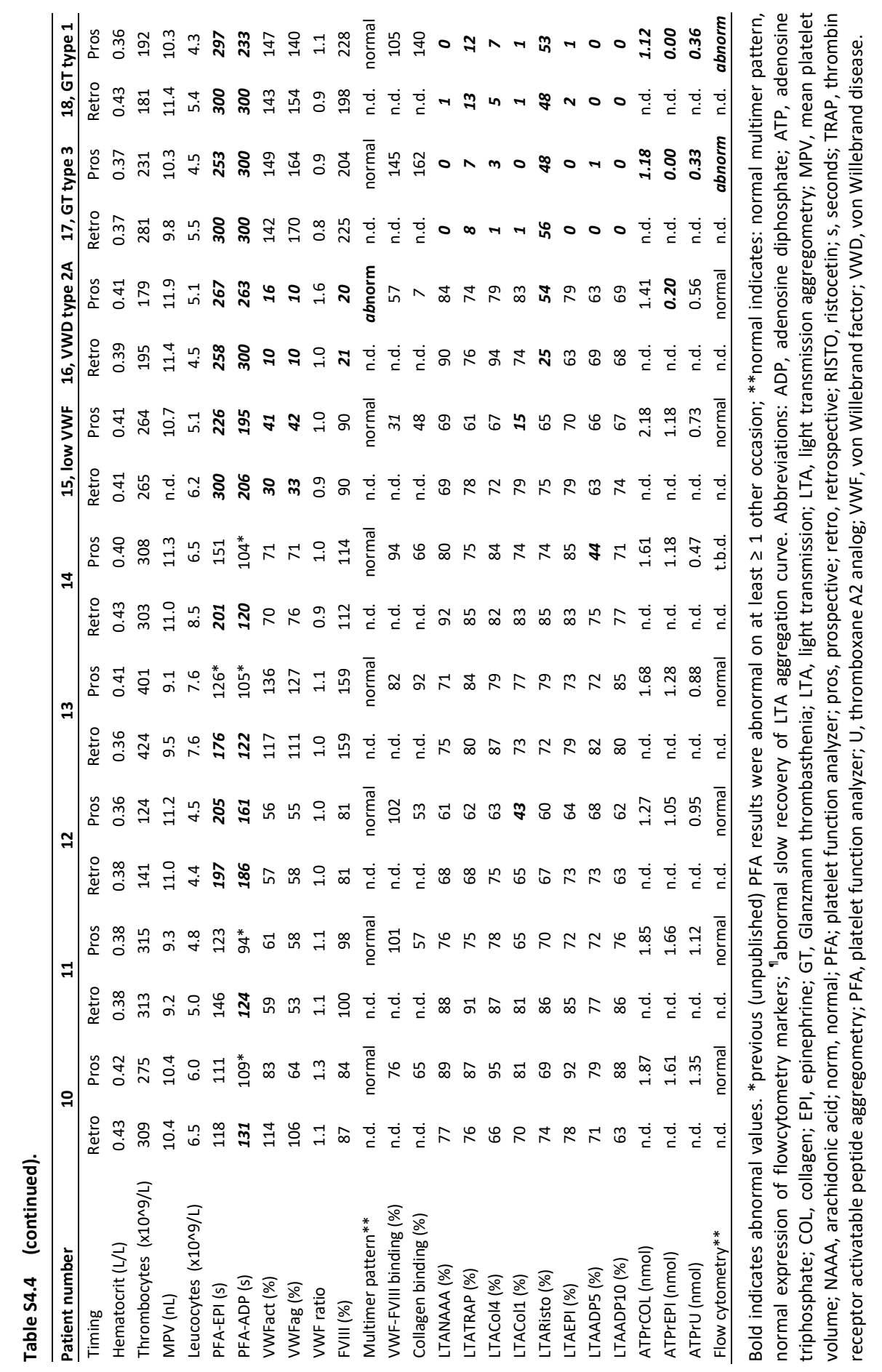




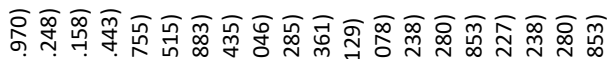

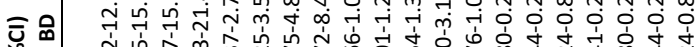

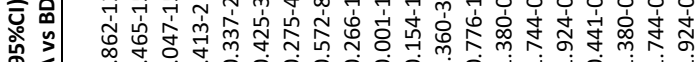

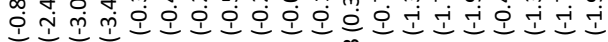

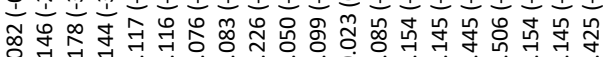

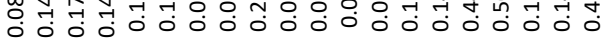

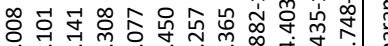



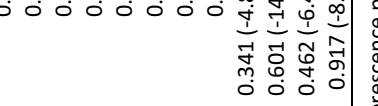

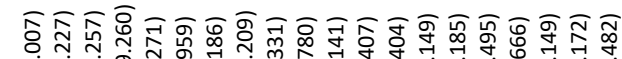

可

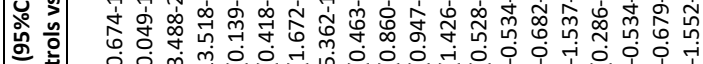

2

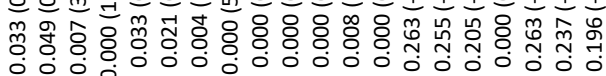

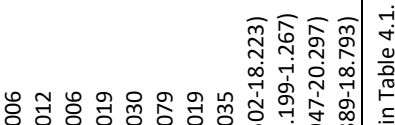

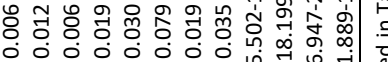

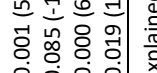

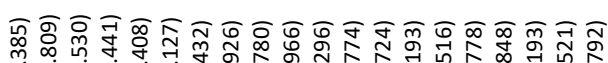

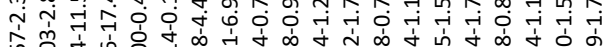

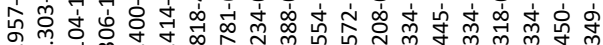

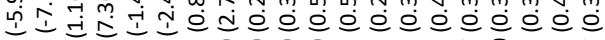

이의.

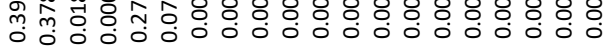

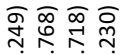

ㄱำ

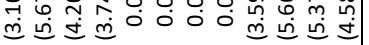

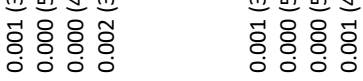

ฐฺุ

ร.ำ

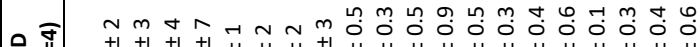

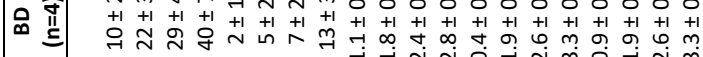

र्่行

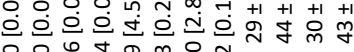

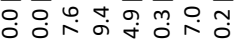

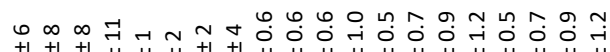

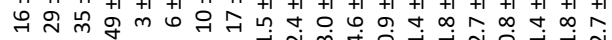

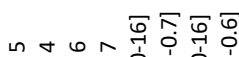

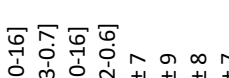

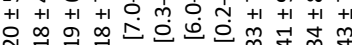

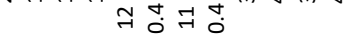

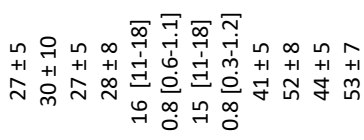

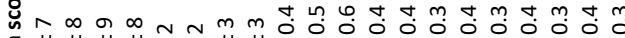

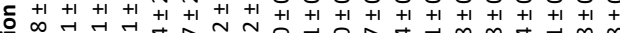

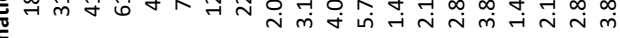

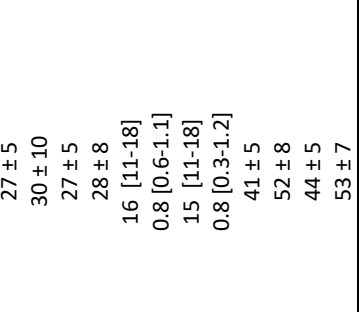

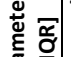

急

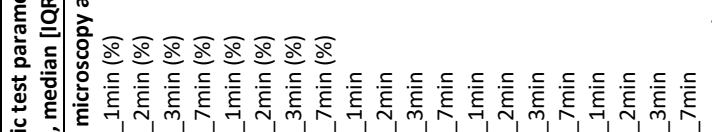

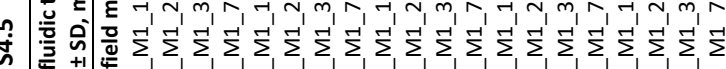

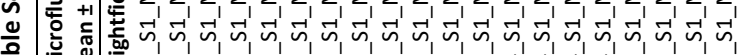

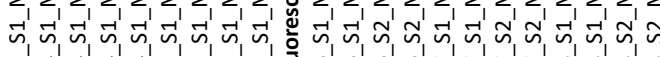

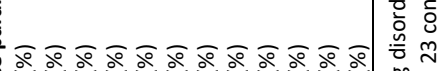

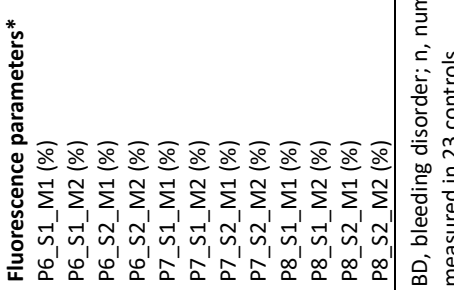




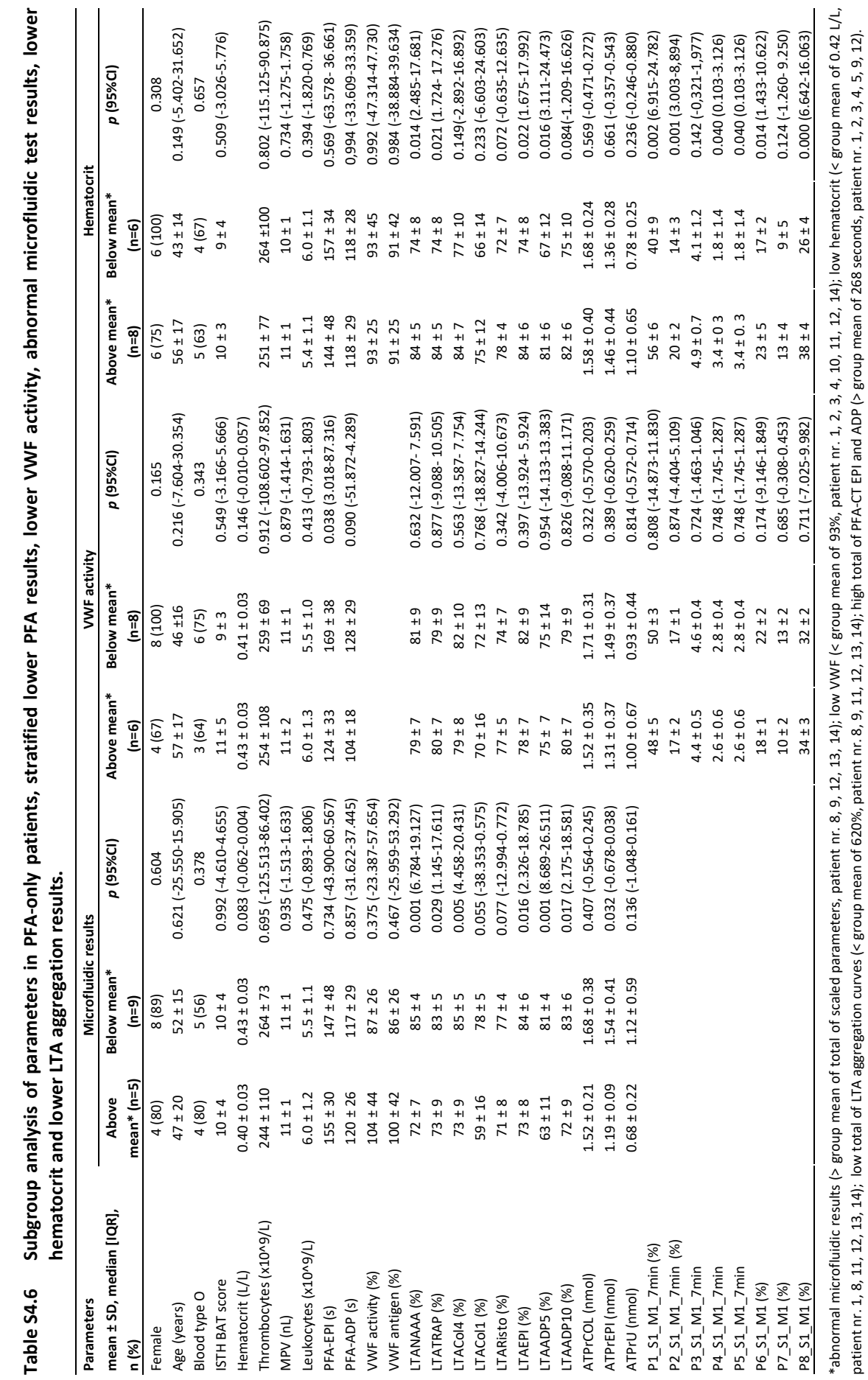




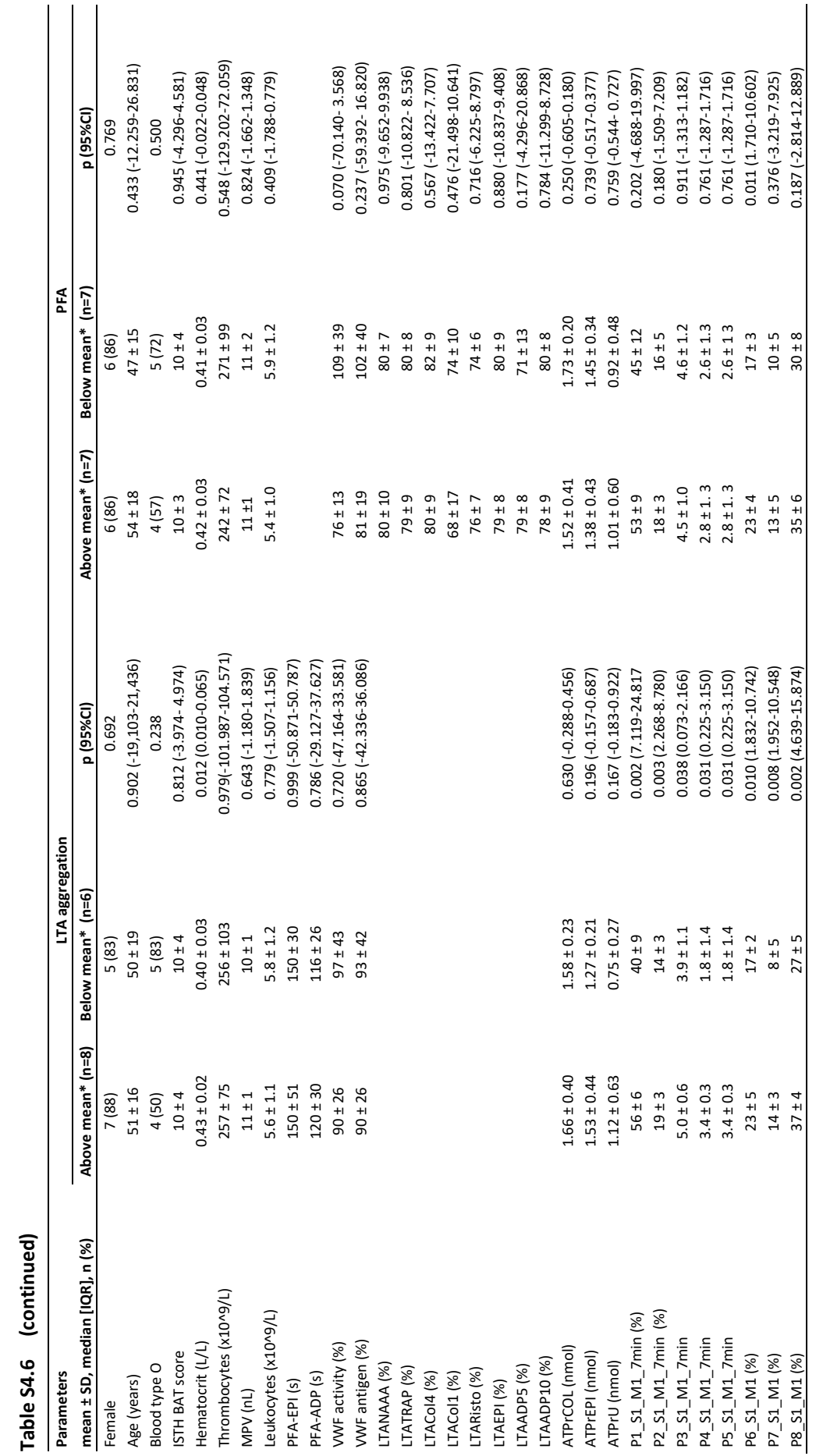




\section{Appendix 4.C Supplemental figures}

Figure S4.1 Correlation analysis to reveal interactions between microfluidic parameters, hematology, PFA, platelet function tests, age, sex, and BAT score in all subjects $(n=68)$.

A

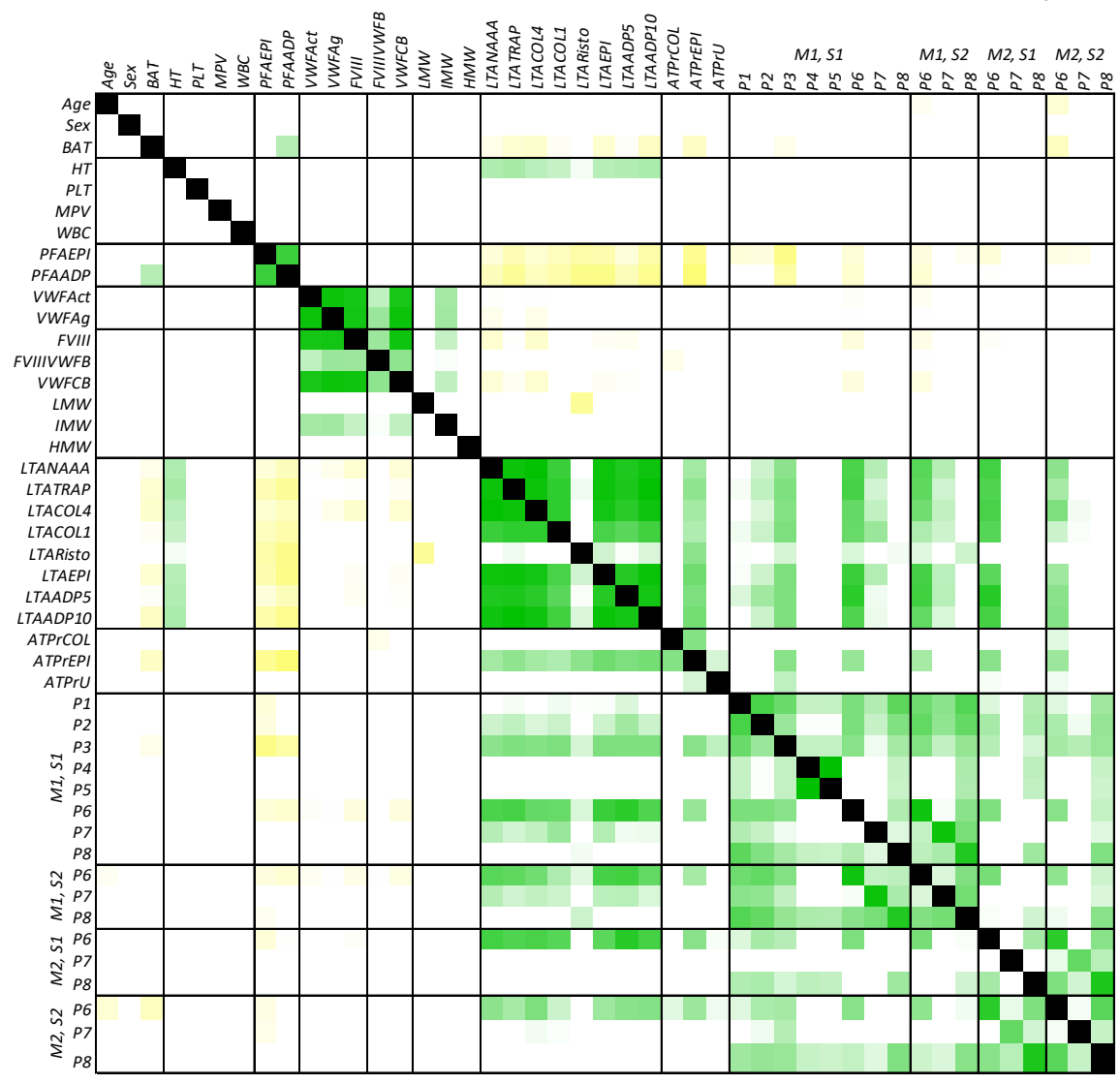

Heat mapped correlations (R) with $p$ values, between microfluidic parameters and subject parameters in all subjects. (A) Heat mapped Pearson correlation coefficients $R$ : a darker color indicates a stronger negative (yellow) or positive (green) correlation. (B) Heat mapped $\mathrm{p}$ values in which a darker color (blue) indicates a highly significant correlation (white offset at $p=0.05$ ). Number of patients $(n)$ for the different comparisons are given. ADP, adenosine diphosphate; ATPr, adenosine triphosphate release; BAT, bleeding assessment tool score; COL, collagen; EPI, epinephrine; HT, hematocrit; LTA, light transmission aggregometry; MPV, mean platelet volume; NAAA, arachidonic acid; PFA; platelet function analyzer; PLT, platelet count; R, correlation coefficient; RISTO, ristocetin; TRAP, thrombin receptor activatable peptide aggregometry; PFA, platelet function analyzer; $U$, thromboxane $A 2$ analog; WBC, white blood cell count; VWFact, von Willebrand factor activity; VWFag, von Willebrand factor antigen. For detailed coding of parameters $(P)$, microspots $(M)$ and shear rates $(S)$ see Table 4.1. 
B

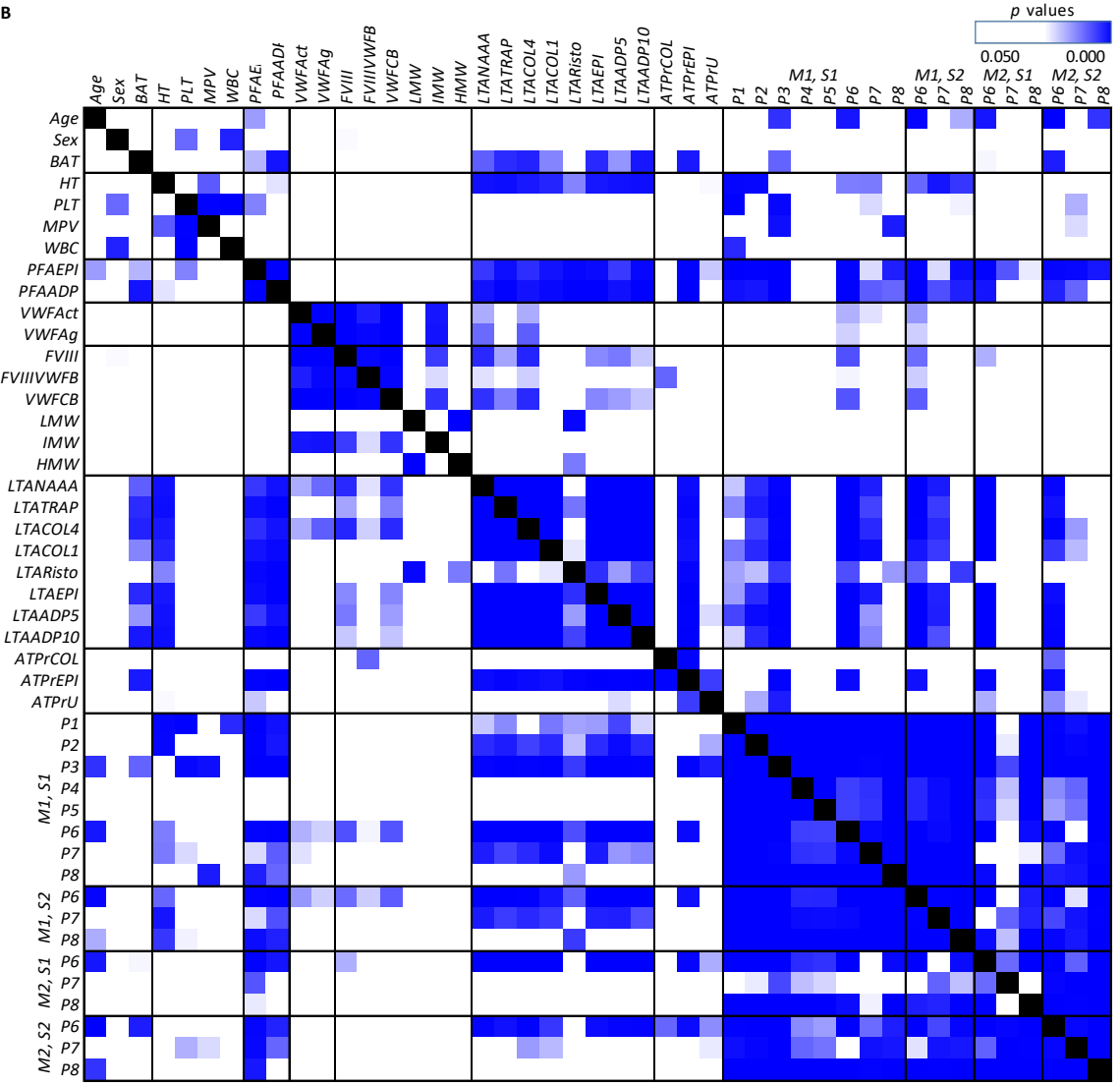

Number of patients for comparison of: age, sex, hematology, $n=68^{*}$; ISTH BAT score, $n=18^{* *}$; PFA, $n=36^{\text {" }}$; VWF, FVIII, VWFCB, VWFFVIIIB, LTA and ATP release, $\mathrm{n}=18^{* *}$; P1-5_M1_S1, $\mathrm{n}=68^{*} ; \mathrm{P} 6-8$-M1_S1/S2, $\mathrm{n}=39^{*}$; P6-8_M2_S1/S2, $n=36^{\S} .{ }^{*}=14$ PFA-only patients, 4 patients with a bleeding disorder, 50 controls; ${ }^{* *}=14$ PFA-only patients, 4 patients with a bleeding disorder; $\uparrow=14$ PFA-only patients, 4 patients with a bleeding disorder, 18 controls; $¥=14$ PFA-only patients, 3 patients with a bleeding disorder, 22 controls; $\S=13$ PFAonly patients, 4 patients with a bleeding disorder, 19 controls. 



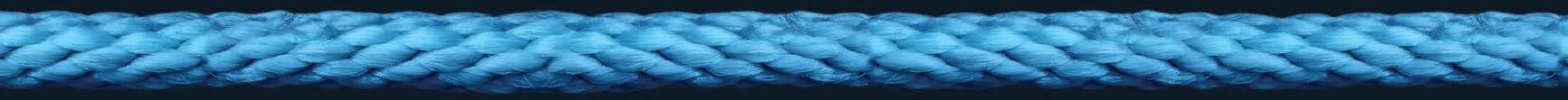




\section{CHAPTER 5}

Effectiveness and costs of a stepwise versus an all-in-one approach to diagnose mild bleeding disorders

Floor C.J.I. Heubel-Moenen, Loes E.M. Ansems, Paul W.M. Verhezen, Rick J.H. Wetzels, Rene G.M. van Oerle, Ron J.M.H.E. Straat, Karyn Megy, Kate Downes, Yvonne M.C Henskens, Erik A.M. Beckers, Manuela A. Joore 


\section{Abstract}

\section{Introduction}

The diagnostic work-up of patients referred to the hematologist for bleeding evaluation is conventionally performed in a stepwise way, in which bleeding history and the results of initial screening laboratory tests guide further evaluation. This can be ineffective, time consuming and burdensome for patients. To improve this diagnostic strategy, the initial laboratory investigation can be extended.

\section{Aim}

To compare the effectiveness and costs of a conventional stepwise versus a newly proposed all-in-one diagnostic approach for bleeding evaluation.

\section{Methods}

In a model-bases analysis, adult patients referred to a hematologist for bleeding evaluation, were evaluated according to a conventional stepwise and an all-in-one diagnostic approach, in which specialized platelet function tests, coagulation factors, and fibrinolysis tests were included in the initial laboratory investigation. Data were collected on diagnostic yield (proportion of patients with a conclusive diagnosis), hospital resource use and costs (doctor visits, multidisciplinary team (MDT) meetings, laboratory costs) and patient burden (hospital visits, venipunctures, travel expenses and time to diagnosis).

\section{Results}

150 patients ( $80 \%$ female, mean age 46 years) were included. Compared to the stepwise approach, in the all-in-one approach, 19 additional patients reached a conclusive diagnosis and patient burden was lower, but total costs per patient were higher (€359, 95\%BCl 283-518, $p=0.001)$.

\section{Conclusions}

For bleeding evaluation of patients referred to the hematologist, an all-in-one diagnostic approach had a higher diagnostic yield and reduced patient burden, but at a higher cost. These findings open the discussion to the value of a conclusive diagnosis in bleeding patients. 


\section{Introduction}

Investigation for a possible bleeding disorder is warranted when a patient presents with a personal and/or family history of bleeding, or with laboratory abnormalities, suggesting a bleeding disorder ${ }^{1}$. In clinical practice, diagnosing patients with a suspected a bleeding disorder is challenging. Experts have proposed and described different guidelines for the diagnostic work-up of patients with increased bleeding tendency ${ }^{1-4}$. These diagnostic algorithms are aimed to help guide rational testing for both common and rare causes of bleeding such as von Willebrand disease (VWD), inherited platelet function disorders (PFD), coagulation factor deficiencies or disorders of fibrinolysis.

Following the experts opinions, patients usually undergo a stepwise diagnostic route. At first, an extensive bleeding history is taken, aided by a standardized bleeding questionnaire and bleeding score, a so-called bleeding assessment tool (BAT) ${ }^{5}$. A proper analysis of family history and use of medication and supplements are also essential elements ${ }^{3}$. Next, laboratory work-up is started with screening coagulation tests. The clinical suspicion for a bleeding disorder, inspired by the bleeding history, the BAT score, together with the results of the screening tests, guide further laboratory evaluation ${ }^{1,2,4,6}$. Although for some tests stored frozen plasma can be used, patient visits are often required more than once. Moreover, abnormal results need confirmation by repeated or even extended laboratory evaluation. The patient's bleeding history and subsequent laboratory tests results are discussed several times in a multidisciplinary team (MDT) with hematologists and laboratory experts, to decide on the next step in laboratory testing. Finally, a definite diagnosis and a patientspecific treatment plan are established.

Following this stepwise approach, the diagnostic trajectory of bleeding evaluation is time consuming and resource-intensive. In case of a clear diagnosis, like type 1 VWD, with a VWF of $20 \%$ or a coagulation factor deficiency, usually 2-3 sample draws and visits are needed to complete all tests ${ }^{1}$. However, if the diagnosis is not straight forward, the patients can be exposed to many venipunctures and hospital visits beforehand ${ }^{2}$. This can be a burden for patients and may cause delays in medical intervention (e.g. surgery). At the same time, the diagnostic yield of the conventional work-up for bleeding evaluation is relatively low, since a hemostatic defect is found in only $25-50 \%$ of the patients ${ }^{7-9}$.

Some centers have extended the 'screening' or initial laboratory investigations in order to reduce the number of venipunctures and hospital visits ${ }^{1}$. Furthermore, new diagnostic tests are introduced into clinical practice to increase the diagnostic yield of bleeding evaluation ${ }^{10-12}$. Finally, genetic testing (single gene or high-throughput 
sequencing (HTS) gene panel testing) is increasingly used to confirm a diagnosis, and inform patients about the genetic nature of their disease. However, the clinical relevance of many genetic variants is not well known, and interpretation of genetic variants should be done carefully, taking into account the phenotype of the patient and the possibility of unsolicited findings ${ }^{13,14}$. The effectiveness and costs of such strategies and tests have not been investigated yet.

To evaluate a new diagnostic test or strategy a cost-effectiveness analysis (CEA) can be done to characterize the costs relative to the amount of benefit that it yields ${ }^{15}$. In this study, diagnostic yield (proportion of patients with a conclusive diagnosis), hospital resource use and costs, and patient burden (e.g. number of venipunctures, time to diagnosis, and travel expenses) were assessed $d^{16,17}$.

\section{Objectives}

The aim of this study was to gain more insight in the diagnostic yield, hospital resource use and costs, patient burden, and cost-effectiveness of the diagnostic workup of patients referred to the hematologist for evaluation of bleeding symptoms. In a model-based analysis, two algorithms, the conventional stepwise approach and a proposed all-in-one approach, were compared using information from an observational patient cohort study. Furthermore, the added diagnostic information of the ThromboGenomics HTS gene panel test results performed at the end of the diagnostic work-up in all patients was evaluated.

\section{Materials and methods}

\section{Study population and dataset}

For this study, data from all patients included in the observational 'Predictors of Bleeding Evaluation in Adult Hematologic Patients with Bleeding Tendencies' (ProBeAHP) study was used. This observational study included adult patients referred to the hematologist for evaluation of bleeding symptoms from September 2015 until December 2020. Details on study design were previously published ${ }^{18}$ and described in Appendix 3.A.

In all patients an International Society of Thrombosis and Hemostasis Bleeding Assessment Tool (ISTH BAT) score was obtained and a large panel of in-hospital laboratory tests was done. A detailed description of blood collection, performed laboratory tests and test procedures is provided in the supplementary methods in Appendix 5.A. 
Patients were sequenced using the ThromboGenomics HTS panel test of diagnostic-grade genes known to harbor variants associated with rare bleeding, thrombotic, or platelet disorders. The ThromboGenomics HTS test sample preparation, sequencing protocols, tested genes, variant prioritization, interpretation and reporting, were extensively described before ${ }^{13,19}$.

\section{Diagnostic algorithms}

In the model-based analysis, two diagnostic algorithms were compared: a conventional stepwise approach, and an in-hospital proposed all-in-one approach. Figure 5.1 represents a simplified display of both diagnostic algorithms. The main difference between both algorithms is the extend of the initial laboratory investigation. The detailed algorithms with decision rules on patient flow and additional laboratory testing are shown in Appendix 5.C Figures S5.1 and S5.2.

The conventional stepwise approach reflects the usual in hospital work-up for bleeding evaluation ${ }^{1-4}$ : during the first appointment at the outpatient hematology department, the patient's medical history including an ISTH BAT score is taken and blood is drawn for screening tests (CBC, aPTT, PT, fibrinogen, thrombin time, PFA-200, VWFact/ant). Frozen plasma is stored. Results of the screening tests and the patients bleeding history are discussed in an MDT. Based on these results, the decision is made to perform additional laboratory tests or to end the diagnostic work-up. The cycle of additional laboratory testing and discussing the results in an MDT is repeated until a final diagnosis is established or patients are classified as bleeders of unknown cause (BUC).

In the all-in-one approach, the initial laboratory investigation, after the first appointment with the hematologist, is extended with more specialized platelet function tests, all coagulation factors and fibrinolysis tests. Results of the bleeding history and all laboratory investigations are discussed in the MDT. Abnormal results are repeated, and if necessary extended, until a final diagnosis is established or patients are classified as BUC.

In both algorithms, single gene analysis was performed in case of a factor VIII deficiency (for family consultation), and in case of VWD type 2 (to confirm the diagnosis). Gene panel analysis was performed in patients with BUC when bleeding score was above 10 (to unravel clinical phenotype) and in patients with macrothrombocytopenia (to confirm diagnosis). 


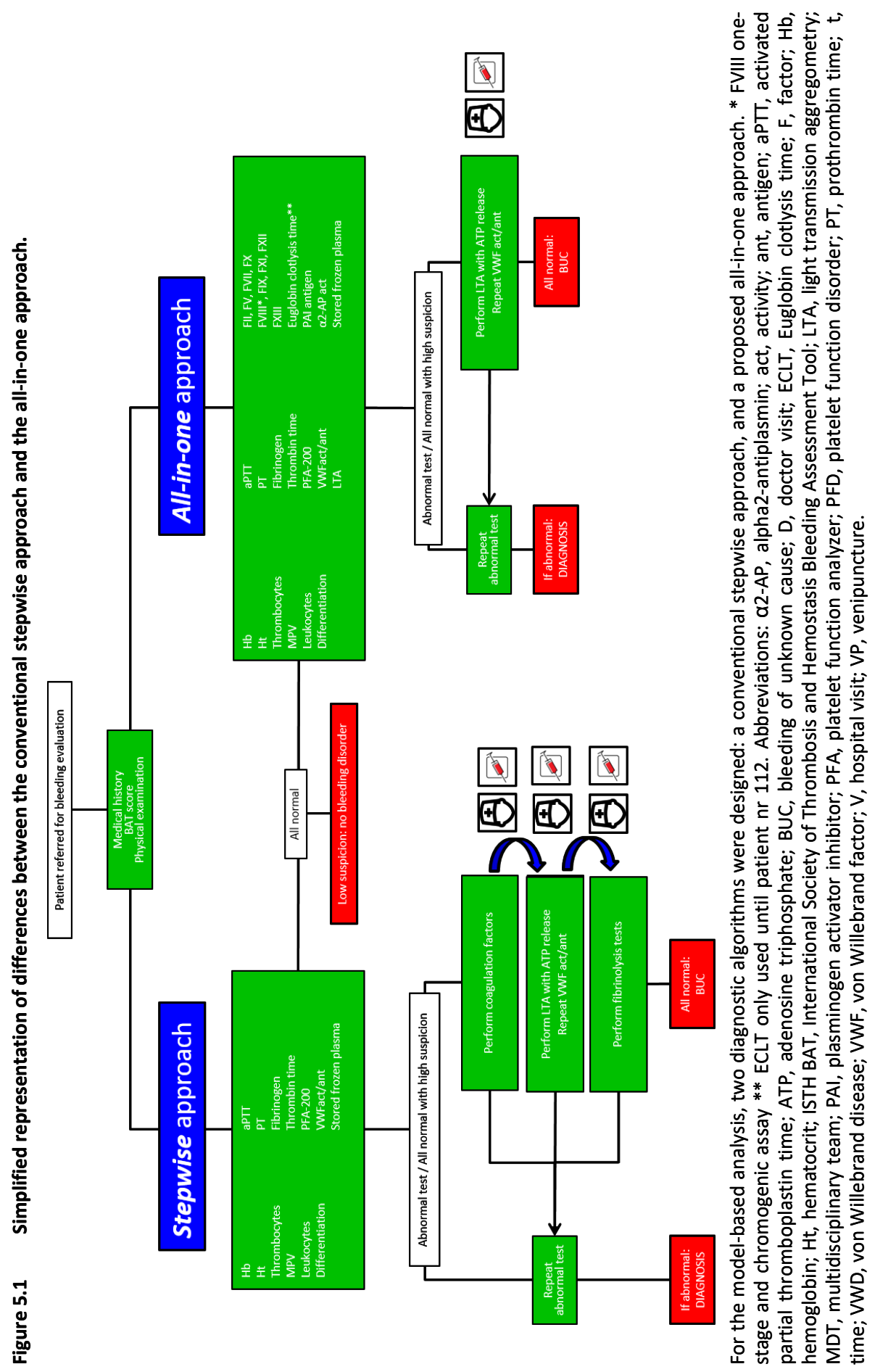




\section{Analysis}

The parameters of the cost and effectiveness analysis are shown in Table 5.1. Two investigators (a medical student and a junior hematologist), independently from each other, evaluated the patient data according to the conventional stepwise approach and the all-in-one approach. Diagnostic criteria for each bleeding disorder were preset, based on literature ${ }^{11,20-23}$ and on our own hospital references and shown in Appendix 5.B Table S5.1. Disagreements in final diagnosis were discussed and agreed on in the MDT.

Table 5.1 Characteristics and parameters of the cost-effectiveness analysis for bleeding evaluation.

\begin{tabular}{ll}
\hline Characteristic & Parameter \\
\hline Diagnostic assessment strategies & Algorithm 1: stepwise approach versus \\
& Algorithm 2: all-in-one approach \\
Effectiveness outcomes & Final diagnosis per patient \\
& Diagnostic yield: proportion of patients with a conclusive \\
& diagnosis \\
Hospital resource use and costs & Doctor visits (number and costs) \\
& MDT meetings (number and costs) \\
& Laboratory costs (blood withdrawal, tests and personnel costs) \\
Indicators of patient burden & Number of hospital visits \\
& Number of venipunctures \\
& Time to diagnosis \\
& Travel expenses \\
Economic outcomes & Total costs per patient \\
& Total costs per diagnosis \\
\hline
\end{tabular}

MDT, multidisciplinary team.

The primary effectiveness outcome was the diagnostic yield, expressed as the proportion of patients with a conclusive diagnosis. Patients were assigned to the 'conclusive diagnosis' category in both algorithms when they were diagnosed with the exact same bleeding disorder(s) (including no bleeding disorder) in both algorithms. Patients with bleeding of unknown cause in both algorithms were assigned to the 'no conclusive diagnosis' category in both algorithms. Patients in whom a diagnosis or additional diagnoses were found in only one of the algorithms, were assigned to the 'no conclusive diagnosis' category in the algorithm were these diagnoses were missed and the 'conclusive diagnosis' category in the algorithm were these diagnoses were found. Patients were assigned to the 'no conclusive diagnosis' category in both algorithms when they were diagnosed with different bleeding disorder(s) in both algorithms.

For comparison of hospital resource use and costs and patients burden between both algorithms, the following data were collected per patient: for hospital resource 
use, number of doctor visits (hematologist consultation), number of MDT meetings and laboratory tests (including blood withdrawal, test costs and personnel costs); for patient burden: number of hospital visits, number of venipunctures, time to diagnosis and travel expenses. Patient travel expenses, and unit costs for the hematologist consultation and the MDT meetings were derived from the Dutch Healthcare Institution cost guideline ${ }^{24}$. Costs of laboratory tests were derived from a cost list published (for hospital use only) by the Dutch Healthcare Authority (NZa) in $2020^{25}$. Internal prices were used for tests that were not available from this list. Full details of both algorithms, with indication of hospital resource use and patient burden are shown in Appendix 5.C Figures S5.3 and S5.4.

Apart from genetic testing as proposed in the algorithms, all available results from the ThromboGenomics HTS gene panel test were compared with the patient's final diagnosis according to both algorithms in order to evaluate the added diagnostic yield of this test in the same manner as described above.

As cost data are usually highly skewed due to a minority of patients utilizing large amounts of resources, bootstrap simulations with 5,000 replications were performed to estimate the mean costs and 2.5-97.5 percentile interval. Likewise, data on diagnostic yield, hospital resource use and patient burden were bootstrapped. Differences between mean results were tested with a bootstrapped paired t-test. Statistical significance was assumed at $p$ value $<0.05$.

An incremental cost-effectiveness ratio (ICER) informing on the costs for an additional patient with a conclusive diagnosis was calculated by dividing the mean difference in costs per patients between both algorithms by the mean difference in diagnostic yield per patient. This represents the extra costs that need to be made to gain one additional patient with a conclusive diagnosis. To address the uncertainty around costs and effects, the costs and diagnostic yield were bootstrapped with 5,000 replications ${ }^{26}$. Results of the CEA were visualized in a CEA plane. Statistical analyses were performed with IBM SPSS statistics version 25, Microsoft excel 2016 and GraphPad Prism 5.

\section{Results}

\section{Baseline characteristics}

Data from a total of 150 patients were available. Baseline characteristics are shown in Table 5.2. Mean age was $46 \pm 17$ years, $80 \%$ of the patients was female. Patients were mostly referred by another specialist in the hospital (52\%), and evaluation of bleeding symptoms was the most common reason for referral (75\%). Median ISTH BAT score 
was 6 [IQR 3-10]. ThromboGenomics HTS gene panel test was performed in 98/150 patients $(65 \%)$.

\section{Effectiveness of the diagnostic algorithms}

Table 5.3 shows the effectiveness of both algorithms. Detailed information about the total number of final diagnoses according to each algorithm is presented in Appendix 5.B Table S5.2.

With the stepwise approach, a total of 52/150 (35\%) patients were diagnosed with one or more bleeding disorders, in 54/150 (36\%) patients there was no bleeding disorder and $44 / 150$ (29\%) patients were diagnosed with bleeding of unknown cause. In the all-in-one approach a total of 66/150 (44\%) patients were diagnosed with one or more bleeding disorders, in $40 / 150(27 \%)$ patients there was no bleeding disorder and $44 / 150$ (29\%) patients were diagnosed with bleeding of unknown cause.

Table 5.2 Patients characteristics $(n=150)$.

\begin{tabular}{lc}
\hline Characteristic & mean \pm SD, median [IQR], $\mathbf{n}(\%)$ \\
\hline Age (years) & $46 \pm 17$ \\
Female & $120(80)$ \\
Referrer & \\
General practitioner & $35(23)$ \\
Other specialist & $78(52)$ \\
Other hospital & $37(25)$ \\
Reason of referral & \\
Bleeding symptoms & $113(75)$ \\
Abnormal laboratory test & $27(18)$ \\
Family investigation & $10(7)$ \\
ISTH BAT score & $6[3-10]$ \\
Blood type O & $82(55)$ \\
Exome sequencing & $98(65)$ \\
No contributing variants found & $82(84)$ \\
Total number of contributing variants found & 16 patients \\
$\quad$ Variant of uncertain significance & 11 \\
Likely pathogenic & 2 \\
Pathogenic & 7 \\
\hline
\end{tabular}

IQR, interquartile range; ISTH BAT, International Society of Thrombosis and Hemostasis Bleeding Assessment Tool; SD, standard deviation; $n$, number. 
Table 5.3 Effectiveness outcomes of the conventional stepwise approach and an all-in-one approach for bleeding evaluation.

\begin{tabular}{lccr}
\hline Final diagnosis per patient & $\begin{array}{c}\text { Stepwise approach } \\
\text { n patients (\%) }\end{array}$ & $\begin{array}{c}\text { All-in-one approach } \\
\text { n patients (\%) }\end{array}$ & $\begin{array}{c}\text { Differences } \\
\text { n patients (\%) }\end{array}$ \\
\hline No bleeding disorder & $54(36)$ & $40(27)$ & $14(9)$ \\
Bleeding of unknown cause & $44(29)$ & $44(29)$ & $0(0)$ \\
Single bleeding disorder & $50(33)$ & $60(40)$ & $14(9)$ \\
Platelet function disorder & $18(12)$ & $26(17)$ & $8(5)$ \\
Factor deficiency & $9(6)$ & $9(6)$ & $0(0)$ \\
von Willebrand disease & $7(5)$ & $6(4)$ & $1(0)$ \\
Fibrinolysis disorder & $12(8)$ & $15(10)$ & $3(2)$ \\
Hypofibrinogenemia & $1(1)$ & $1(1)$ & $0(0)$ \\
Macrothrombocytopenia & $3(2)$ & $3(2)$ & $0(0)$ \\
Multiple diagnoses & $2(1)^{\dagger}$ & $6(4)^{\ddagger}$ & $4(3)$ \\
\hline Diagnostic yield* & $\mathbf{4 7 ( 3 1 )}$ & $\mathbf{6 6 ( 4 4 )}$ & $\mathbf{1 9 ( 1 3 ) * *}$ \\
\hline
\end{tabular}

* Diagnostic yield: proportion of patients with a conclusive diagnosis. ** 14 patients with a conclusive diagnosis in the all-in-one approach, not found in the stepwise approach and 5 patients with one or two additional diagnoses in the all-in-one approach, not found in the stepwise approach. See Table 5.4. † Diagnoses: VWD type 1 and FXI deficiency; VWD type 1 and low fibrinogen.

₹ Diagnoses: VWD type 1 and FXI deficiency; VWD type 1 and low fibrinogen and PFD and fibrinolysis disorder. VWD type 2 and PFD; FV deficiency and fibrinolysis disorder; FVII deficiency and fibrinolysis disorder; FXI deficiency and fibrinolysis disorder.

F, factor; n, number; PFD, platelet function disorder; VWD, von Willebrand disease

The bootstrapped diagnostic yield was $58 \%$ for the stepwise approach and $71 \%$ for the all-in-one diagnostic approach (Figure 5.2). In the all-in-one approach, a conclusive diagnosis was established in 19/150 (13\%) additional patients (Table 5.4). In five female patients diagnosed with a bleeding disorder in the stepwise approach, 1 (four patients) or 2 (one patient) additional diagnoses were found in the all-in-one approach (2 platelet function disorders, 4 fibrinolysis disorders). These patients all had a high ISTH BAT score $(\geq 11)$. The other 14 patients were classified as having no bleeding disorder in the stepwise approach, but diagnosed with a PFD (8/14), FVIII deficiency (2/14), FVII deficiency (1/14) and a fibrinolysis disorder (3/14) in the all-inone approach. ISTH BAT score was normal in all of these patients. Patient 13 was referred because of bleeding symptoms and a positive family history for hemophilia $A$ (his mother was carrier of hemophilia A). In the stepwise approach this was not diagnosed because he had a normal BAT score and the APTT was normal (30 sec), although his FVIII level was $27 \%$. There were no patients with a conclusive diagnosis in the stepwise approach, not detected in the all-in-one approach. 
Figure 5.2 Diagnostic yield of the conventional stepwise approach and the all-in-one approach.

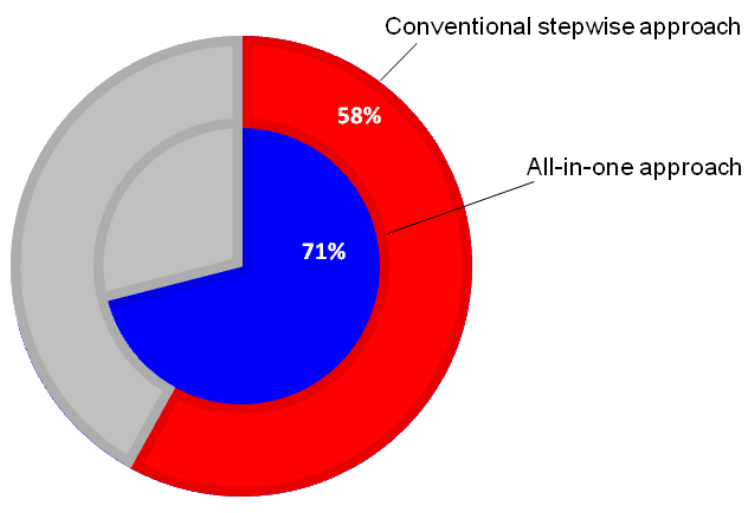

In the stepwise approach $58 \%$ of the patients received a conclusive diagnosis, in the all-one-approach $71 \%$ were diagnosed with a conclusive diagnosis

\section{Hospital resource use and costs and patient burden}

Hospital resource use and costs and patient burden for both algorithms are shown in Table 5.5. Non-bootstrapped data were comparable and are available in Appendix 5.B Table S5.3. Hospital resource use, costs and patients burden per diagnosis are presented in Appendix 5.B Tables S5.4 and S5.5.

The bootstrapped mean number of doctor visits and MDT meetings were significantly lower in the all-in-one approach compared to the stepwise approach (doctor visits 3 vs. 4, $p=0.001$; MDT meetings 2 vs. 3, $p=0.001$ ). On the other hand, bootstrapped mean laboratory costs were significantly higher in the all-in-one approach (€1,544 vs. $€ 987, p=0.001)$. With regard to patient burden, the bootstrapped mean number of venipunctures was lower and time to diagnosis was shorter in the all-in-one approach compared to the stepwise approach (venipunctures 2 vs. $3, p=0.001$; time to diagnosis 11 vs. 16 weeks, $p=0.001$ ). There was no significant difference in bootstrapped mean number of hospital visits and patients travel costs in the all-in-one approach compared to the stepwise approach (hospital visits 4 vs. 5, $p=0.250$, travel costs $€ 26$ vs. $€ 26, p=0.287)$. 


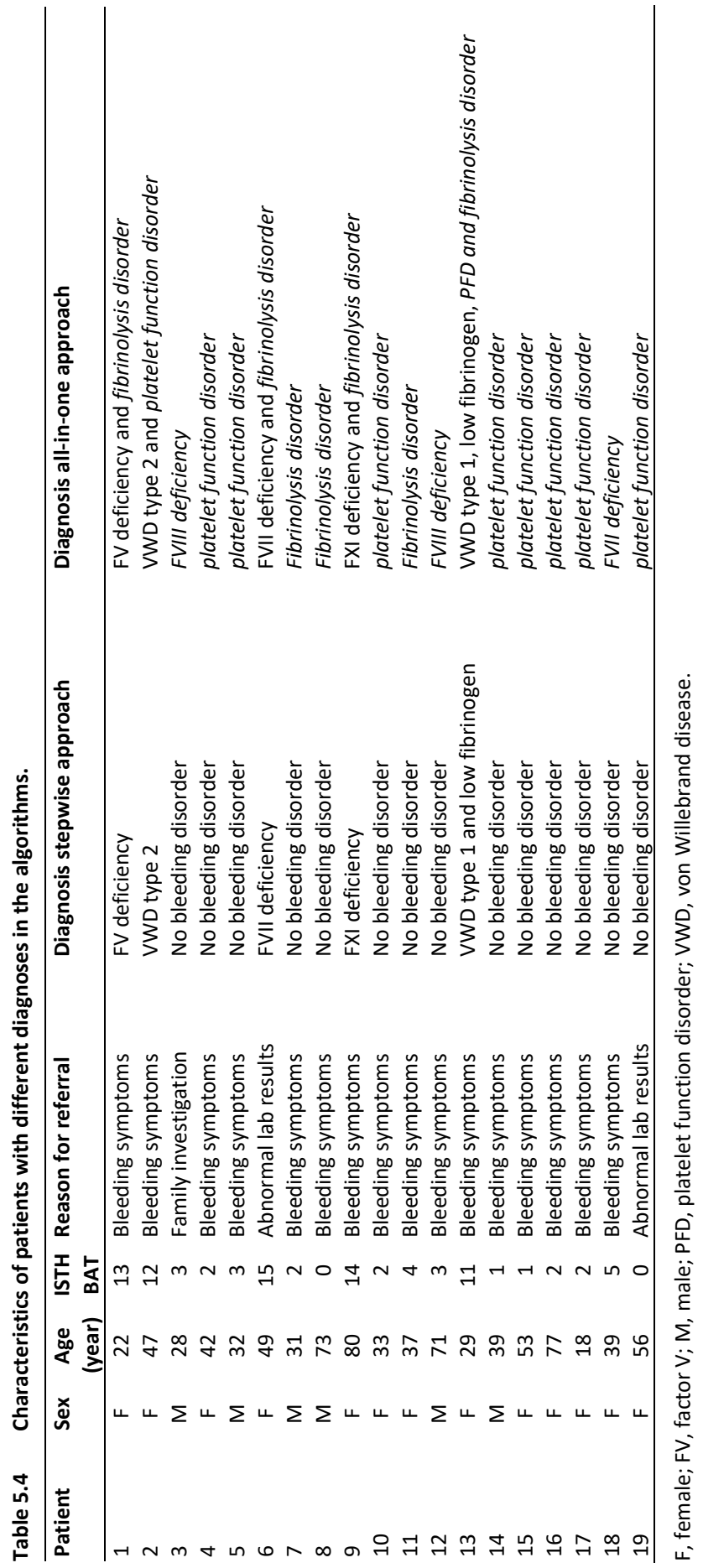




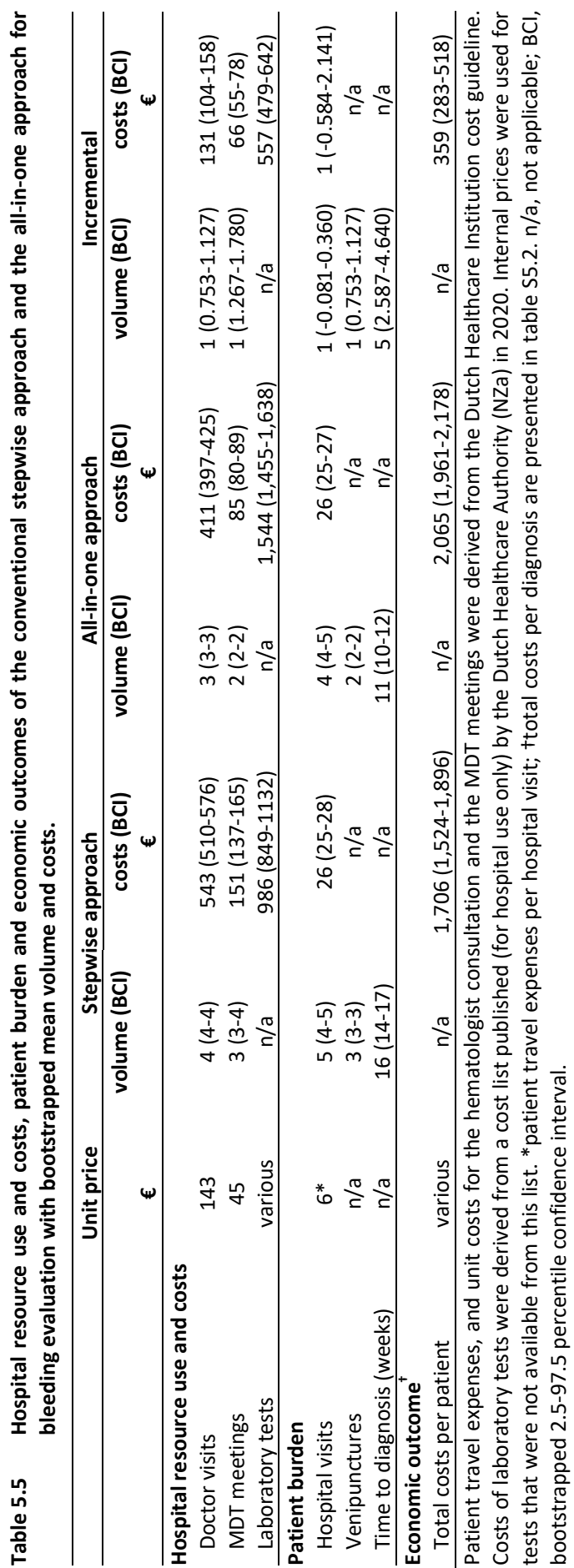




\section{Cost-effectiveness}

Total bootstrapped mean costs per patient were higher in the all-in approach $(€ 2,065)$ than in the stepwise approach $(€ 1,706, p=0.001)$. The mean incremental bootstrapped costs amounted to $€ 359$ ( $95 \% \mathrm{BCl} 283-518)$. The increment in bootstrapped diagnostic yield was $0.13(95 \% \mathrm{BCl} 0.120-0.133)$. This results in an ICER of $€ 2,830$ per additional patient with a conclusive diagnosis. Figure 5.3 shows the uncertainty surrounding the incremental costs and diagnostic yield, based on the bootstrap analysis. In $99 \%$ of the bootstrap stimulations the all-in-one diagnostic approach was more costly but yielded more patients with a conclusive diagnosis.

Figure 5.3 CEA plane.

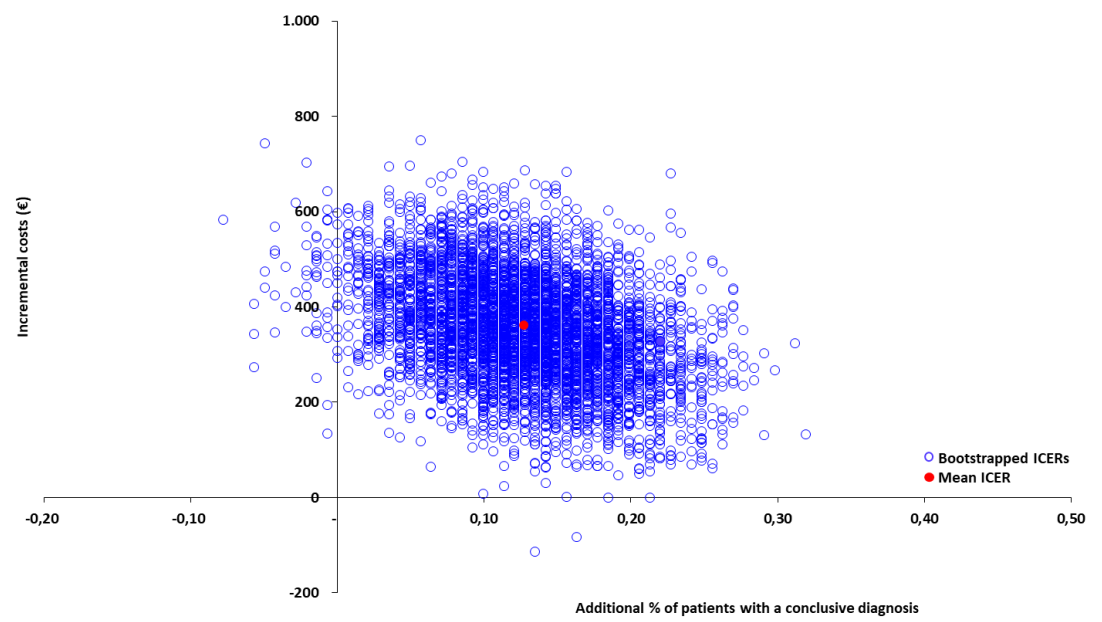

Incremental cost-effectiveness ratio (ICER) of the all-in-one diagnostic approach compared to the conventional stepwise approach measured as costs per additional patient with a conclusive diagnosis.

\section{ThromboGenomics HTS gene panel test}

98/150 (65\%) of the patients were sequenced using the ThromboGenomics HTS panel test of diagnostic-grade genes known to harbor variants associated with rare bleeding, thrombotic, or platelet disorders ${ }^{13}$.

Table 5.6 shows the 20 genetic variants that were found in 16/98 (16\%) patients: 7 pathogenic variants (3 F11 mutations, 3 F8 mutations, 1 RUNX1 mutation); 2 likely pathogenic variants (1 VWF mutation, 1 PTPN11 mutation); and 11 variants of unknown significance (1 F5 mutation, 1 F8 mutation, 1 VWF mutation, 1 GFI1B mutation, 1 NBEAL2 mutation, 2 P2PY12 mutations, 2 ITGB3 mutations and 2 ACTN1 mutations). 


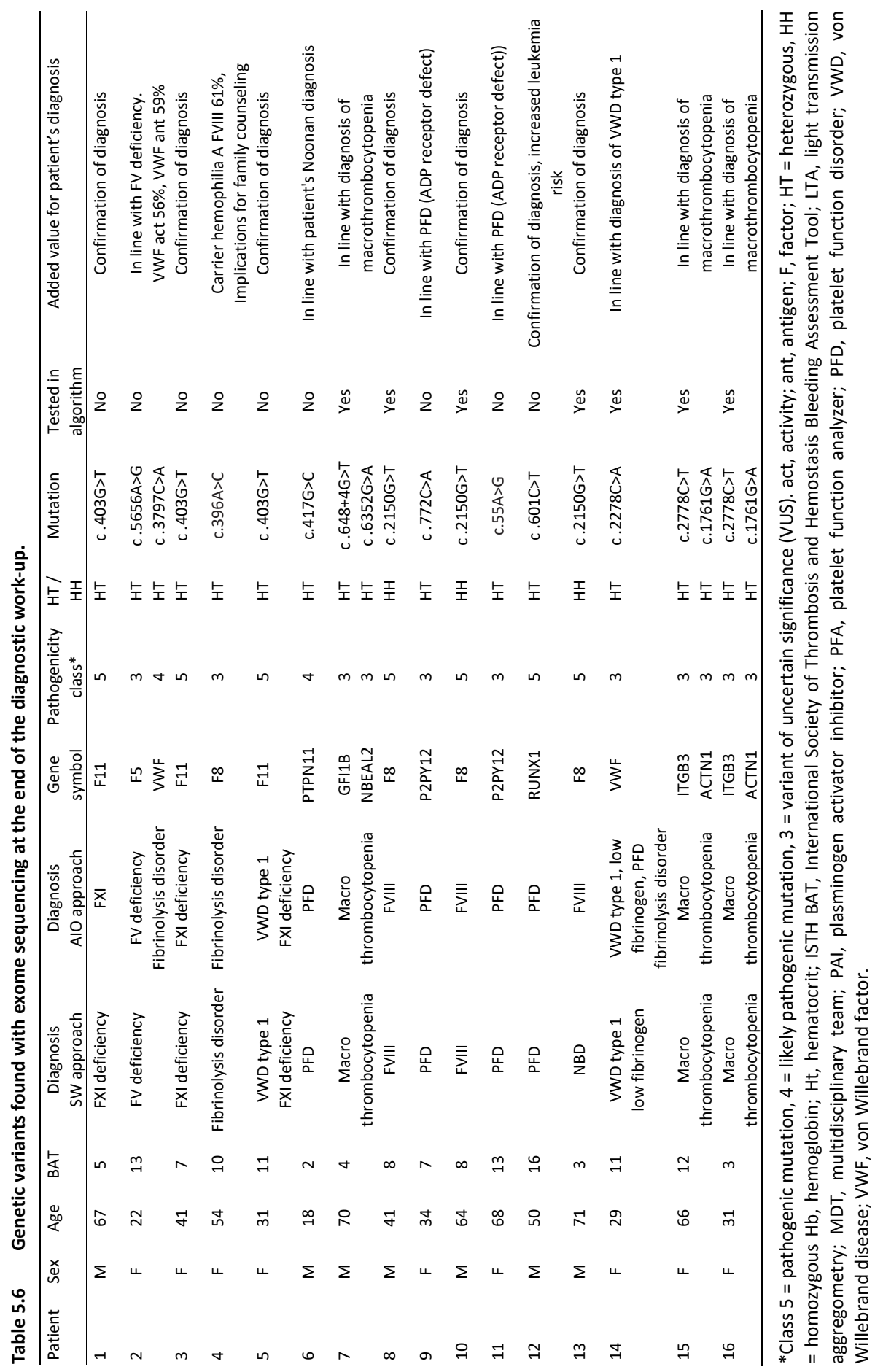


The pathogenic variants confirmed the already established diagnoses in all cases. According to both algorithms, only the F8 gene would have been sequenced, so the pathogenic FXI and RUNX1 mutations would not have been found.

The likely pathogenic variant in VWF was found in a patient who was diagnosed with FV deficiency (FV 40\%, a VUS in F5 was found as well) and a PAI deficiency (PAI ant $3.3 \mathrm{ng} / \mathrm{mL}$ ). VWF act. and ant. were $56 \%$ resp. 59\% in this patient. Lower VWF levels might contribute to bleeding in this young patient with a high bleeding score of 13. The likely pathogenic mutation in PTPN11 was in line with the patient's diagnosis of Noonan syndrome, with a PFD.

$10 / 11$ VUS were in line with the established diagnoses. In one female patient, diagnosed with a fibrinolysis disorder, a VUS was found in the F8 gene, indicating carriership for hemophilia A. Her FVIII level was $61 \%$. Both of her sons were diagnosed with mild hemophilia A and the same F8 mutation was found.

\section{Discussion}

This is, to our knowledge, the first study to investigate the diagnostic yield, hospital resource use and costs, patient burden, and cost-effectiveness of different diagnostic algorithms for bleeding evaluation in patients referred to the hematologist with a bleeding tendency. Our results show that the proposed all-in-one diagnostic approach, compared to the conventional stepwise approach, has a $13 \%$ higher diagnostic yield. Furthermore, the all-in-one diagnostic approach results in less hospital resource use and costs with regard to doctor visits and MDT meetings, and in less venipunctures and a shorter time to diagnosis. However, due to the higher laboratory costs, the mean total costs per patient of this all-in-one approach are higher (€359) compared to the conventional stepwise approach. This resulted in an ICER of $€ 2,830$. A genetic variant was found in $16 / 98$ of the patients evaluated with the ThromboGenomics HTS gene panel test.

In the all-in-one approach 19 additional patients reached a conclusive diagnosis. In five of these patients, all with high bleeding scores, multiple diagnoses were established. The additional hemostatic abnormalities might contribute to the bleeding symptoms in these patients. The total number of patients with multiple bleeding disorders was $6 / 150(4 \%)$ in the all-in-one approach compared to $2 / 150(1 \%)$ in the stepwise approach. The percentage found in the all-in-one approach is more in agreement with a previous study investigating mucocutaneous bleeding in 280 patients, were the frequency of combined hemostatic defects was $5 \%(13 / 280)^{7}$. The occurrence of severe bleeding, or a high bleeding score in a patient diagnosed with a 
mild bleeding disorder, should therefore always raise the consideration of additional hemostatic defects. Extensive laboratory investigation is justified in these patients.

In 14 patients with no bleeding disorder according to the stepwise approach (negative ISTH BAT score and normal screening tests), a mild bleeding disorder was found after performing extensive laboratory testing in the all-in-one approach. This emphasizes our previous finding that a negative bleeding score is not able to rule out a mild bleeding disorder ${ }^{27}$. Performing extensive laboratory testing in all patients, regardless of the bleeding score, overcomes these limitations. However, care should be taken when the diagnosis of the bleeding disorder is solely based on laboratory testing, unless the qualifying laboratory assay has a very high likelihood for a distinct disorder ${ }^{4}$. Another advantage of establishing the conclusion of 'no bleeding disorder' after extensive laboratory testing is that this might be more reassuring, compared to when only screening tests are performed.

The incremental cost-effect ratio (ICER), or the costs per additional patient with a conclusive diagnosis, amounted to $€ 2,830$. This raises the question what costs justify a conclusive diagnosis in a patient suspected of a bleeding disorder and a diagnostic strategy that is less burdensome for patients.

Regarding the value of a conclusive diagnosis, patients with mild bleeding disorders do not have an increased mortality, but they may suffer from increased morbidity, like uncontrolled bleeding after surgery, trauma or post-partum ${ }^{28-30}$. Also, bleeding symptoms can be perceived as alarming and have impact on work and school participation (e.g. heavy menstrual bleeding) ${ }^{31}$. After a conclusive diagnosis, preventive treatment during menstrual cycle or peri-operative can be advised ${ }^{32}$. A successful preventive treatment reassures the patient and his family, prevents morbidity, and enables full participation in school and working life ${ }^{4}$. Furthermore, establishing a targeted treatment plan for a specific bleeding disorder can prevent hospital admission because of bleeding or prolonged hospital stay due to bleeding after surgery or delivery, saving hospital resources and costs.

Regarding patient burden, in the all-on-one approach compared to the stepwise approach, less doctor visits, less venipunctures, and shorter time to diagnosis, is likely of value to the patient. A visit to the doctor implies taking leave from work or school, affecting job or school performances. Venipunctures can cause anxiety in patients. Although fear of needles is most common in children, about $15-30 \%$ of (female) adults (aged 20-40 years) report fear of needles, and 16\% avoids medical procedures because of this ${ }^{33,34}$. Shorter time to diagnosis results in shorter time with untreated bleeding, improving quality of life of the patient. It also leads to less delay, moving and cancelation of dental or surgical procedures. We believe that these advantages justify the additional costs in the all-in-one approach. Furthermore, shorter time to 
diagnoses also has financial consequences for the health system. In the Dutch health care system, payment of healthcare is based on a diagnosis treatment combination system (DBC) defined by the Dutch Healthcare Authority. At first visit to the hospital, a DBC code is registered and declared at the patient's health insurance. The first DBC has a term of 90 days, after which a new DBC, with a term of 120 day is registered and declared $^{35}$. Shorter time to diagnosis means less registered DBCs, saving money from both the patient and the healthcare system. All these significant additional benefits were not quantified in our analysis.

Although we did not evaluate quality of life, it is still interesting to compare the ICER of $€ 2,830$ with the costs we are willing to pay for a quality-adjusted life year (QALY). In the Netherlands, it is generally found acceptable to spend between $€ 20,000$ to $€ 80,000 / \mathrm{QALY}^{36}$. The low end of this range applies to situations with a relatively low burden of disease, including prevention ${ }^{24}$. This would mean that in our study, the mean additional diagnostic yield per patient of $13 \%$ would need to translate in at least a mean of 0.018 QALY gained per patient (or 0.14 QALY gained per patient with a conclusive diagnosis).

A genetic variant was found in 16/98 of the patients evaluated with the ThromboGenomics HTS gene panel test. No variants were found in patients with bleeding of unknown cause. This was in line with previous publication on prevalence of genetic mutations found in different patient groups ${ }^{13}$. All but two variants confirmed the conclusive diagnosis. The two unexpected variants were reviewed. In one patient a likely pathogenic variant in VWF was found, while her VWF activity was $56 \%$ (reference range $50-150 \%$ ). In this case, repeated VWF measures would be justified, to rule out elevation of VWF by stress or as an acute phase protein. In the other patient a VUS in factor FVIII was found, while she was diagnosed with a fibrinolysis disorder. Her FVIII level was 61\% (reference range 60-150\%). However, since her sons were diagnosed with hemophilia A (FVIII level 30\%), having the same F8 variant, this patient is an obligate carrier of hemophilia $A$.

This study has some important limitations. First, we focused on costs and diagnostic yield, but did not quantify the impact of a conclusive diagnosis on the quality of life and survival of the patient. Preferably, a final outcome such as qualityadjusted-life-year (QALY) is used in cost-effectiveness analysis. Measuring the value that patients put on reassurance when they do (or do not) receive a diagnosis, and the effect on symptoms, quality of life and survival when a proper treatment is installed is feasible and subject for future studies ${ }^{37}$. Second, family history was not taken into account in both diagnostic algorithms, but would have at least explained the discrepancy between the algorithms in one of the patients. Furthermore, an algorithm 
is never able to fully reflect the daily practice of bleeding evaluation, since it misses nuances in clinical judgement and MDT discussion.

The finding of a pathogenic RUNX1 mutation puts forward ethical issues that can occur with gene panel sequencing for platelet function disorders. Although this variant confirms the diagnosis of macrothrombocytopenia found in this patient, it is also associated with an increased lifetime risk of leukemia of $45 \%^{38}$. Close surveillance and family counseling is consequently warranted. Variant interpretation was performed in the context of the clinical phenotype of the patient. Therefore, it was not feasible to explore a 'DNA-first' diagnostic approach were results of exome sequencing were available before other laboratory tests were performed.

In conclusion, for the evaluation of bleeding symptoms in patients referred to the hematologist, an all-in-one diagnostic approach, compared to the conventional stepwise approach, has a higher diagnostic yield and leads to lower patient burden, at higher costs of $€ 359$ per patient. In our hospital, this led us to change the work-up of patients referred for bleeding evaluation from a step-wise approach to an all-in-one approach. For future research we would like to further investigate the impact on (long-term) quality of life and costs, and the role of upfront DNA analyses in patients suspected with bleeding tendencies. 


\section{References}

1. Hayward CPM. How I investigate for bleeding disorders. Int J Lab Hematol 2018;40 Suppl 1:6-14.

2. Boender J, Kruip MJ, Leebeek FW. A diagnostic approach to mild bleeding disorders. J Thromb Haemost 2016;14(8):1507-16.

3. Zegers SAM, et al. Diagnostic work up of patients with increased bleeding tendency. Haemophilia 2020;26(2):269-77.

4. Rodeghiero F, et al. Fundamentals for a Systematic Approach to Mild and Moderate Inherited Bleeding Disorders: An EHA Consensus Report. Hemasphere 2019;3(4):e286.

5. Rydz N, James PD. The evolution and value of bleeding assessment tools. J Thromb Haemost 2012; 10(11):2223-9.

6. Gebhart J, et al. The discriminatory power of bleeding assessment tools in adult patients with a mild to moderate bleeding tendency. Eur J Intern Med 2020;78:34-40.

7. Quiroga $\mathrm{T}$, et al. High prevalence of bleeders of unknown cause among patients with inherited mucocutaneous bleeding. A prospective study of 280 patients and 299 controls. Haematologica 2007; 92(3):357-65.

8. Gebhart J, et al. High proportion of patients with bleeding of unknown cause in persons with a mildto-moderate bleeding tendency: Results from the Vienna Bleeding Biobank (VIBB). Haemophilia 2018;24(3):405-13.

9. Thomas W, Downes K, Desborough MJR. Bleeding of unknown cause and unclassified bleeding disorders; diagnosis, pathophysiology and management. Haemophilia 2020.

10. van Asten I, Schutgens REG, Urbanus RT. Toward Flow Cytometry Based Platelet Function Diagnostics. Semin Thromb Hemost 2018;44(3):197-205.

11. Hayward CPM, et al. Update on diagnostic testing for platelet function disorders: What is practical and useful? Int J Lab Hematol 2019;41 Suppl 1:26-32.

12. Potgieter JJ, Damgaard M, Hillarp A. One-stage vs. chromogenic assays in haemophilia A. Eur J Haematol 2015;94 Suppl 77:38-44.

13. Downes K, et al. Diagnostic high-throughput sequencing of 2396 patients with bleeding, thrombotic, and platelet disorders. Blood 2019;134(23):2082-91.

14. Downes K, et al. Clinical management, ethics and informed consent related to multi-gene panel-based high throughput sequencing testing for platelet disorders: Communication from the SSC of the ISTH. J Thromb Haemost 2020;18(10):2751-8.

15. Shi CR, Nambudiri VE. Research Techniques Made Simple: Cost-Effectiveness Analysis. J Invest Dermatol 2017;137(7):e143-7.

16. Graziadio S, et al. How to Ease the Pain of Taking a Diagnostic Point of Care Test to the Market: A Framework for Evidence Development. Micromachines (Basel) 2020;11(3).

17. Black WC. The CE plane: a graphic representation of cost-effectiveness. Med Decis Making 1990; 10(3):212-4.

18. Moenen FCJ, et al. Screening for platelet function disorders with Multiplate and platelet function analyzer. Platelets 2019;30(1):81-7.

19. Megy $\mathrm{K}$, et al. Curated disease-causing genes for bleeding, thrombotic, and platelet disorders: Communication from the SSC of the ISTH. J Thromb Haemost 2019;17(8): 1253-60.

20. Leebeek FW, Eikenboom JC. Von Willebrand's Disease. N Engl J Med 2016;375(21):2067-80.

21. Saes JL, et al. Hemorrhagic disorders of fibrinolysis: a clinical review. J Thromb Haemost 2018.

22. de Moerloose P, Casini A, Neerman-Arbez M. Congenital fibrinogen disorders: an update. Semin Thromb Hemost 2013;39(6):585-95.

23. Verhovsek M, Moffat KA, Hayward CP. Laboratory testing for fibrinogen abnormalities. Am J Hematol 2008;83(12):928-31.

24. Nederland Z. Kosteneffectiviteit in de praktijk, Z. Nederland, Editor. 2015.

25. Nederland Z., Richtlijn voor het uitvoeren van economische evaluaties in de gezondheidszorg, in Kostenhandleiding. 2016, Institute for Medical Technology Assessment Erasmus Universiteit Rotterdam. 
26. van Hout BA, et al. Costs, effects and C/E-ratios alongside a clinical trial. Health Econ 1994;3(5):30919.

27. Moenen F, et al. The diagnostic accuracy of bleeding assessment tools for the identification of patients with mild bleeding disorders: A systematic review. Haemophilia 2018;24(4):525-35.

28. Quiroga T, Mezzano D. Is my patient a bleeder? A diagnostic framework for mild bleeding disorders. Hematology Am Soc Hematol Educ Program 2012;2012:466-74.

29. Greaves M, Watson HG. Approach to the diagnosis and management of mild bleeding disorders. J Thromb Haemost 2007;5 Suppl 1:167-74.

30. Orsini $S$, et al. Bleeding risk of surgery and its prevention in patients with inherited platelet disorders. Haematologica 2017;102(7):1192-203.

31. Blumenthal PD, Dawson L, Hurskainen R. Cost-effectiveness and quality of life associated with heavy menstrual bleeding among women using the levonorgestrel-releasing intrauterine system. Int J Gynaecol Obstet 2011;112(3):171-8.

32. Vries MJ, et al. Preoperative screening for bleeding disorders: A comprehensive laboratory assessment of clinical practice. Res Pract Thromb Haemost 2018;2(4):767-77.

33. McLenon J, Rogers MAM. The fear of needles: A systematic review and meta-analysis. J Adv Nurs 2019;75(1):30-42.

34. Deacon B, Abramowitz J. Fear of needles and vasovagal reactions among phlebotomy patients. J Anxiety Disord 2006;20(7):946-60.

35. Zorgautoriteit N., Handleiding dbc-systematiek RZ20a v20181220. 2020.

36. Ministerie van Volksgezondheid, W.e.S., Ziektelast in de praktijk - De theorie en praktijk van het berekenen van ziektelast bij pakketbeoordelingen.

37. Mushlin Al, Ruchlin HS, Callahan MA. Costeffectiveness of diagnostic tests. Lancet 2001;358(9290): 1353-5.

38. Galera P, Dulau-Florea A, Calvo KR. Inherited thrombocytopenia and platelet disorders with germline predisposition to myeloid neoplasia. Int J Lab Hematol 2019;41 Suppl 1:131-41. 


\section{Appendix 5.A Supplementary methods}

\section{Blood collection}

Patients were asked to avoid fat containing food 4 hours before blood withdrawal, as plasma lipids might interfere with LTA measurements. Patients had to stop any interfering medication (e.g. NSAIDs) 7 days before blood withdrawal. Blood was drawn from the antecubital vein by puncture using a $21 \mathrm{G}$ needle. Complete blood count was measured in EDTA blood ( $1,8 \mathrm{mg} / \mathrm{mL}, \mathrm{BD}$ Vacutainer, Plymouth).

Platelet-rich plasm (PRP) was obtained by centrifuging citrated blood at $170 \mathrm{~g}$ for $10 \mathrm{~min}$ at $18^{\circ} \mathrm{C}$. For the preparation of platelet free plasma (PFP) citrated-blood was centrifuged at $10000 \mathrm{~g}$ for $10 \mathrm{~min}$ at $18^{\circ} \mathrm{C}$.

\section{Laboratory measurements}

Hemoglobin $(\mathrm{Hb})$, platelet count and white blood cell (WBC) measurements were performed on a Sysmex XN 9000 analyzer (Sysmex Corporation, Kobe, Japan). PT (Innovin, Siemens), APTT (ActinFSL, Siemens), thrombin time (Thromboclotin Siemens), fibrinogen (Clauss method, Thrombin Reagent, Siemens) and FXIII activity (Berichrom FXIII, Siemens) measurements were performed on a Sysmex CS 2100i (Sysmex Corporation, Kobe, Japan). Levels of FII, FV, FVII, FX (Thromborel S, Siemens) and FVIII, FIX, FXI (actin FS, Siemens), were measured with a clotting assay using factor-depleted plasma on a Sysmex CS 2100i (Sysmex Corporation, Kobe, Japan). Levels of VWF antigen and activity were determined on a Sysmex CS2100i analyser (VWF Reag and VWF Ac Reagens, Siemens, Sysmex Europe, Norderstedt, Germany. Platelet function was analyzed by the platelet function analyzer 200 (PFA-200; Innovance PFA-200 System, Siemens, Marburg, Germany), closure times (CTs) were recorded with collagen and epinephrine (C-EPI) or collagen and ADP (C-ADP) kits. For assessment of Light Transmission Aggregometry (LTA), a Chronolog aggregometer was used (Chrono-log Corporation, Havertown, USA). Platelet aggregation in PRP was induced with 5 or $10 \mu \mathrm{M}$ ADP (Chronolog CH384), 1 or $4 \mu \mathrm{g} / \mathrm{mL}$ collagen-I (Chrono-par Ref385), $10 \mu \mathrm{M}$ epinephrine (Chronolog CH393), $1 \mathrm{mM}$ arachidonic acid (Bio/Data) $1.5 \mathrm{mg} / \mathrm{mL}$ ristocetin (Chronolog-Stago Ref396), or $15 \mu \mathrm{M}$ thrombin receptor activating peptide (TRAP-6; Boom H8105). For ATP release measurements, platelets were activated by $5 \mu \mathrm{g} / \mathrm{mL}$ collagen, $5 \mu \mathrm{M}$ epinephrine, or $1 \mu \mathrm{M}$ thromboxane $A_{2}$ analog U46619. Reference values for ATP release were 1.20-2.79 nmol for collagen, 0.86-2.47 nmol for epinephrine, and 0.39-1.87 nmol for U46619. The Euglobin clotlysis test was based on a home-made clotlysis method (SOP 031211), reference values were locally established. PAI-1 antigen was measured with Zymutest PAI-A antigen (Hyphen BioMed), PAl-1 activity was measured with the Zymutest PAI-A activity kit (Hypen BioMed). Reference values were locally established. Plasminogen was measured with Berichrom Plasminogen kit (Siemens CS2100), $\alpha 2-A P$ (Stachrom Antiplasmin) was performed by chromogenic assays on Sta-R, (Stago, Paris). 


\section{Appendix 5.B Supplemental tables}

Table S5.1 Diagnostic criteria for bleeding disorders.

\begin{tabular}{|c|c|c|c|}
\hline Disease & Laboratory test & Normal values & Diagnostic criteria \\
\hline VWD type 1 & VWF act/ant, ratio & $50-150 \%,>0.7$ & VWFact and/or ant $<50 \%$, ratio $>0.7$ \\
\hline VWD type 2 & VWF act/ant, ratio & $50-150 \%,>0.7$ & VWFact and/or ant $<50 \%$, ratio $<0.7$ \\
\hline VWD type 3 & VWF act/ant & $50-150 \%$ & VWFact and/or ant $<5 \%$ \\
\hline \multirow[t]{5}{*}{ Platelet function disorder } & $\begin{array}{l}\text { Light transmission } \\
\text { aggregometry }\end{array}$ & $\begin{array}{l}\text { Maximal } \\
\text { aggregation } \\
>60 \%, \text { normal } \\
\text { shape of curve }\end{array}$ & $\begin{array}{l}\geq 2 \text { abnormal aggregations, or } \\
\text { abnormal aggregation with ristocetin } \\
\text { or collagen } 4 \mu \mathrm{g} / \mathrm{mL}\end{array}$ \\
\hline & ATP release & & $\geq 1$ clear abnormalities \\
\hline & collagen & $1.20-2.79 \mathrm{nmol}$ & \\
\hline & epinehrine & $0.86-2.47 \mathrm{nmol}$ & \\
\hline & U46619 & $0.39-1.87 \mathrm{nmol}$ & \\
\hline \multirow[t]{2}{*}{ Macrothrombocytopenia } & Smear & $\begin{array}{l}\text { Normal } \\
\text { thrombocytes }\end{array}$ & Macrothrombocytes \\
\hline & Thrombocyte count & $150-350 \times 10^{9} / \mathrm{L}$ & Thrombocyte count $<150 \times 10^{\wedge} 9 / \mathrm{L}$ \\
\hline \multirow[t]{8}{*}{ Factor deficiency } & Factor II & $60-140 \%$ & $<60 \%$ \\
\hline & Factor V & $60-140 \%$ & $<60 \%$ \\
\hline & Factor VII & $60-140 \%$ & $<60 \%$ \\
\hline & Factor VIII & $50-200 \%$ & $<50 \%$ \\
\hline & Factor IX & $60-140 \%$ & $<60 \%$ \\
\hline & Factor $\mathrm{X}$ & $60-140 \%$ & $<60 \%$ \\
\hline & Factor XI & $60-140 \%$ & $<60 \%$ \\
\hline & Factor XIII & $70-140 \%$ & $<70 \%$ \\
\hline Afibrinogenemia & $\begin{array}{l}\text { Clottable/immunoreactive } \\
\text { fibrinogen, ratio }\end{array}$ & $1.6-4.2 \mathrm{~g} / \mathrm{L}, 1$ & $\begin{array}{l}\text { clottable/immunoreactive fibrinogen } \\
\text { undetectable }\end{array}$ \\
\hline Hypofibrinogenemia & $\begin{array}{l}\text { Clottable/immunoreactive } \\
\text { fibrinogen, ratio }\end{array}$ & $1.6-4.2 \mathrm{~g} / \mathrm{L}, 1$ & $\begin{array}{l}\text { clottable/immunoreactive fibrinogen } \\
<1 \mathrm{~g} / \mathrm{L} \text {, ratio around } 1\end{array}$ \\
\hline Dysfibrinogenemia & $\begin{array}{l}\text { Clottable/immunoreactive } \\
\text { fibrinogen, ratio }\end{array}$ & $1.6-4.2 \mathrm{~g} / \mathrm{L}, 1$ & $\begin{array}{l}\text { discrepancy between clottable and } \\
\text { immunoreactive fibrinogen, ratio }>1\end{array}$ \\
\hline \multirow[t]{4}{*}{ Fibrinolysis disorder } & Euglobin clotlysis time & $90-240$ minutes & $\begin{array}{l}<60 \text { minutes } \\
<80 \text { minutes with PAl act }<1.0\end{array}$ \\
\hline & PAI-1 activity & $<10.4 \mathrm{ng} / \mathrm{mL}$ & \\
\hline & PAI-1 antigen & $3.4-39 \mathrm{ng} / \mathrm{mL}$ & $<3.4 \mathrm{ng} / \mathrm{mL}$ with PAl act $<1.0$ \\
\hline & $\alpha 2$-AP activity & $80-130 \%$ & $<80 \%$ \\
\hline Bleeding of unknown cause & No laboratory abnormalities & & $\begin{array}{l}\text { ISTH BAT score } \geq 4 \text { in men, } \geq 6 \text { in } \\
\text { female }\end{array}$ \\
\hline No bleeding disorder & No laboratory abnormalities & & $\begin{array}{l}\text { ISTH BAT score }<4 \text { in men, }<6 \text { in } \\
\text { female }\end{array}$ \\
\hline
\end{tabular}

Act, activity; ant, antigen; ATP, adenosine triphosphate; COL, collagen; EPI, epinephrine; VWD, von Willebrand disease; VWF, von Willebrand factor. 
Table S5.2 Detailed information on final diagnosis and costs per diagnosis according to both approaches.

\begin{tabular}{|c|c|c|}
\hline Diagnosis & $\begin{array}{c}\text { Stepwise approach } \\
\text { n diagnosis (\%) }\end{array}$ & $\begin{array}{c}\text { All-in-one approach } \\
\text { n diagnosis (\%) }\end{array}$ \\
\hline No bleeding disorder & $54(36)$ & $40(26)$ \\
\hline Abnormal PFA & 10 & 6 \\
\hline Bleeding of unknown cause & $44(29)$ & $44(28)$ \\
\hline PFA only & 10 & 10 \\
\hline ISTH BAT >10 & 11 & 11 \\
\hline Platelet function disorder & $18(12)$ & $26(17)$ \\
\hline Based on ATP release & 8 & 8 \\
\hline Factor deficiency & $9(6)$ & $12(8)$ \\
\hline FV & 1 & 1 \\
\hline FVII & 1 & 2 \\
\hline FVIII & 3 & 5 \\
\hline FXI & 4 & 4 \\
\hline von Willebrand disease & $9(6)$ & $9(6)$ \\
\hline VWD type 1 & 8 & 8 \\
\hline VWD type 2 & 1 & 1 \\
\hline Fibrinolysis disorder & $12(8)$ & $19(12)$ \\
\hline ECLT $<60$ minutes & 2 & 5 \\
\hline ECLT $<80$ minutes \& & 4 & 8 \\
\hline \multicolumn{3}{|l|}{ PAl-1 act $<1.0 \mathrm{ng} / \mathrm{mL}$} \\
\hline $\mathrm{PAl}$ antigen $<3.4 \mathrm{ng} / \mathrm{mL}$ & 6 & 6 \\
\hline Hypofibrinogenemia & $2(1)$ & $2(1)$ \\
\hline Macrothrombocytopenia & $3(2)$ & $3(2)$ \\
\hline
\end{tabular}

ATP, adenosine triphosphate; ECLT, Euglobin clotlysis time; F, factor; ISTH BAT, International Society of Thrombosis and Hemostasis Bleeding Assessment Tool; $n$, number; PAl, plasminogen activator inhibitor; PFA, platelet function analyzer; PFD, platelet function disorder; SD, standard deviation; VWD, von Willebrand disease. 
Table S5.3 Hospital resource use and patient burden per diagnosis.

\begin{tabular}{|c|c|c|c|c|c|}
\hline & $\begin{array}{c}\text { Doctor visits } \\
\text { (n) }\end{array}$ & $\begin{array}{l}\text { Hospital visits } \\
\text { (n) }\end{array}$ & $\begin{array}{c}\text { Venipunctures } \\
\text { (n) }\end{array}$ & $\begin{array}{l}\text { MDT meetings } \\
\text { (n) }\end{array}$ & $\begin{array}{c}\text { TTD } \\
\text { (weeks) }\end{array}$ \\
\hline \multicolumn{6}{|l|}{ No bleeding disorder } \\
\hline Stepwise approach & 2 & 2 & 1 & 1 & 6 \\
\hline All-in-one approach & 2 & 3 & 1 & 1 & 6 \\
\hline \multicolumn{6}{|c|}{ Bleeding of unknown cause } \\
\hline Stepwise approach & 5 & 6 & 4 & 5 & 26 \\
\hline All-in-one approach & 3 & 5 & 2 & 2 & 10 \\
\hline \multicolumn{6}{|c|}{ BUC with exome sequencing } \\
\hline Stepwise approach & 6 & 7 & 5 & 6 & 34 \\
\hline All-in-one approach & 4 & 6 & 3 & 3 & 22 \\
\hline \multicolumn{6}{|c|}{ Platelet function disorder } \\
\hline Stepwise approach & 4 & 6 & 3 & 4 & 14 \\
\hline All-in-one approach & 3 & 5 & 2 & 2 & 10 \\
\hline \multicolumn{6}{|l|}{ Factor deficiency } \\
\hline Stepwise approach & 3 & 3 & 2 & 3 & 10 \\
\hline All-in-one approach & 3 & 4 & 2 & 2 & 8 \\
\hline \multicolumn{6}{|c|}{ Factor deficiency with single gene analysis } \\
\hline Stepwise approach & 4 & 4 & 3 & 4 & 22 \\
\hline All-in-one approach & 4 & 5 & 3 & 3 & 20 \\
\hline \multicolumn{6}{|l|}{ von Willebrand disease } \\
\hline Stepwise approach & 3 & 3 & 2 & 2 & 10 \\
\hline All-in-one approach & 3 & 4 & 2 & 2 & 10 \\
\hline \multicolumn{6}{|c|}{ VWD with single gene analysis } \\
\hline Stepwise approach & 4 & 4 & 3 & 3 & 22 \\
\hline All-in-one approach & 4 & 5 & 3 & 3 & 22 \\
\hline \multicolumn{6}{|l|}{ Fibrinolysis disorder } \\
\hline Stepwise approach & 6 & 7 & 5 & 6 & 26 \\
\hline All-in-one approach & 3 & 4 & 2 & 2 & 12 \\
\hline \multicolumn{6}{|c|}{ A/hypo/dysfibrinogenemia } \\
\hline Stepwise approach & 3 & 3 & 2 & 2 & 10 \\
\hline All-in-one approach & 3 & 5 & 2 & 2 & 10 \\
\hline \multicolumn{6}{|c|}{ Macrothrombocytopenia with exome sequencing } \\
\hline Stepwise approach & 5 & 7 & 4 & 5 & 26 \\
\hline All-in-one approach & 4 & 6 & 3 & 3 & 22 \\
\hline
\end{tabular}

BUC, bleeding of unknown cause; MDT, multidisciplinary team; n, number; TTD, time to diagnosis; VWD, von Willebrand disease. 


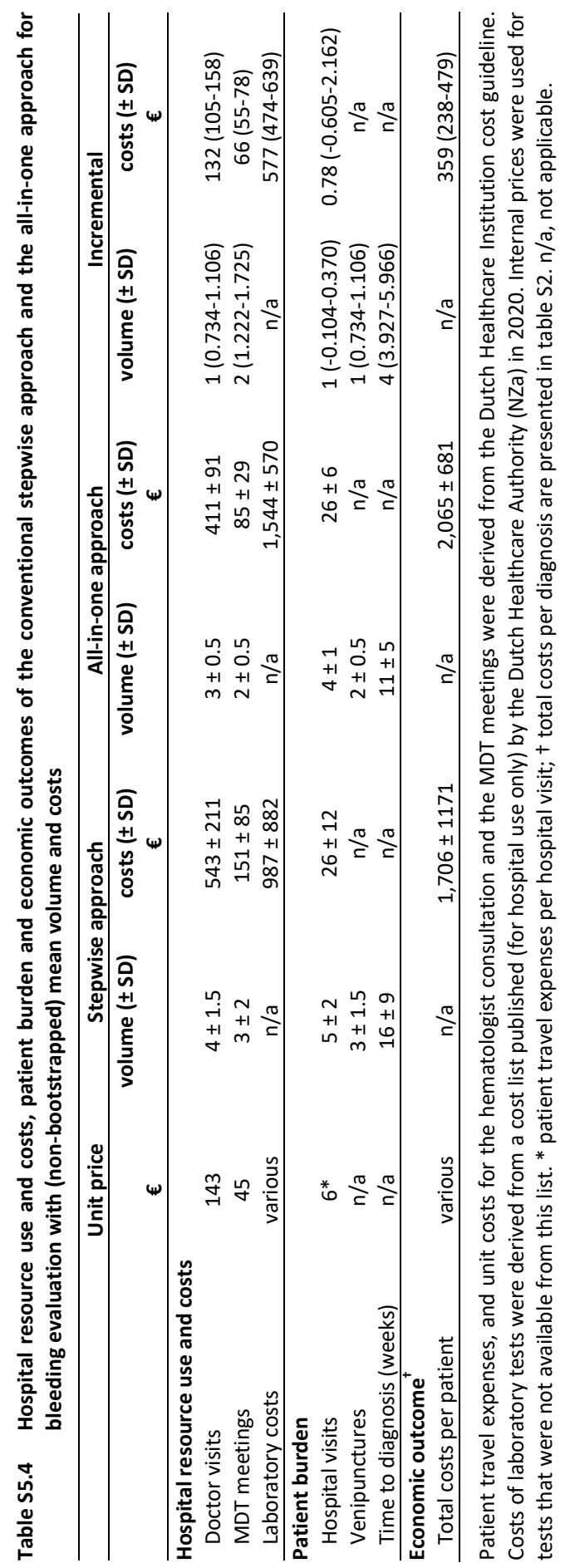


Table S5.5 Bootstrapped mean costs per diagnosis according to both algorithms.

\begin{tabular}{lccc}
\hline Diagnosis & $\begin{array}{c}\text { Stepwise approach } \\
\text { costs }(\mathrm{BCl}) €\end{array}$ & $\begin{array}{c}\text { All-in-one approach } \\
\text { costs }(\mathrm{BCI}) €\end{array}$ & $\begin{array}{c}\text { Incremental } \\
\text { costs }(\mathrm{BCI}) €\end{array}$ \\
\hline No bleeding disorder & $544(505-586)$ & $1431(1415-1448)$ & $887(931-840)$ \\
Bleeding of unknown cause & $2414(2371-2449)$ & $2044(2015-2072)$ & $145(98-356)$ \\
$\quad$ with gene panel testing & $4260(4210-4313)$ & $3834(3737-3912)$ & $426(332-529)$ \\
Platelet function disorder & $1497(1389-1593)$ & $1841(1820-1859)$ & $345(245-457)$ \\
$\quad$ based on ATP release & $1774(1674-1816)$ & $2070(2037-2104)$ & $296(231-387)$ \\
Factor deficiency & $975(867-1061)$ & $1728(1610-1889)$ & $753(573-992)$ \\
$\quad$ with single gene analysis & $2009(1911-2062)$ & $2699(2657-2716)$ & $689(610-792)$ \\
Von Willebrand disease & $1025(797-1492)$ & $2000(1730-2490)$ & $976(331-1561)$ \\
$\quad$ with single gene analysis & 2026 & & \\
Fibrinolysis disorders & $3093(3040-3146)$ & $2182(2160-2204)$ & $911(852-979)$ \\
Hypofibrinogenemia & 742 & 1695 & 889 \\
Macrothrombocytopenia & $3353(3227-3417)$ & $3857(3746-3912)$ & $504(270-737)$ \\
\hline
\end{tabular}

ATP, adenosine triphosphate; $\mathrm{BCl}$, bootstrapped 2.5-97.5 percentile confidence interval. 
Appendix 5.C Supplemental figures

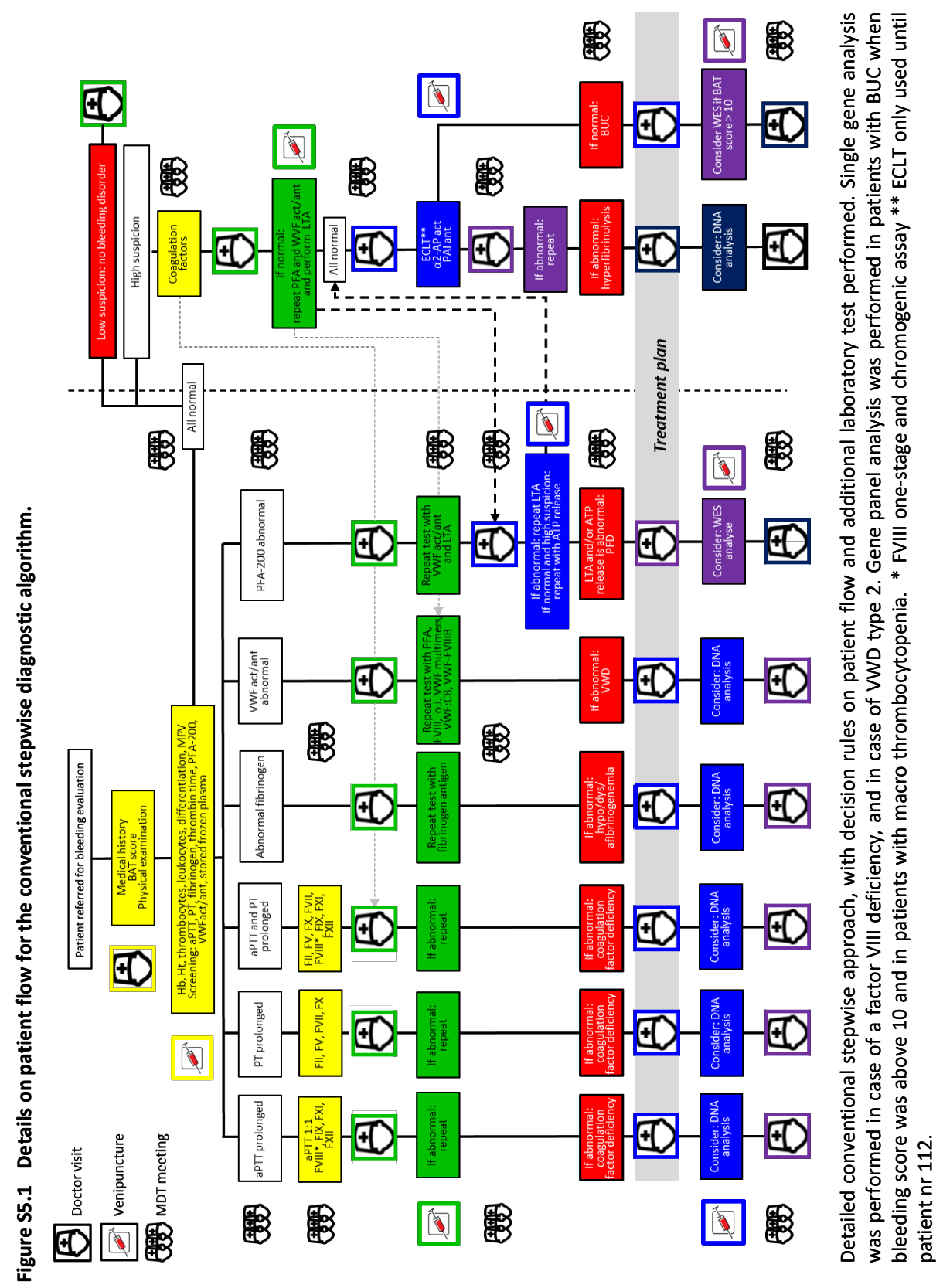




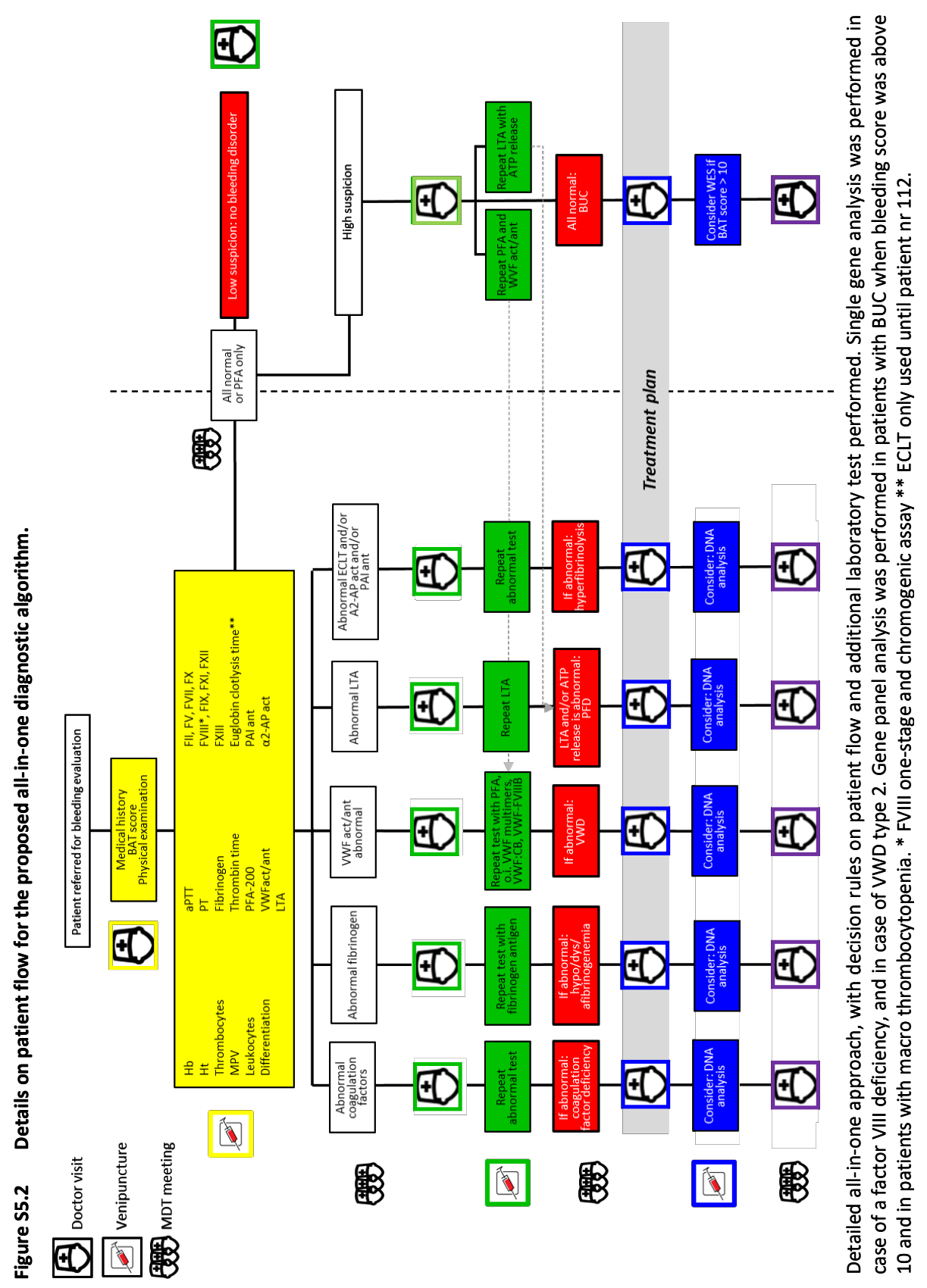




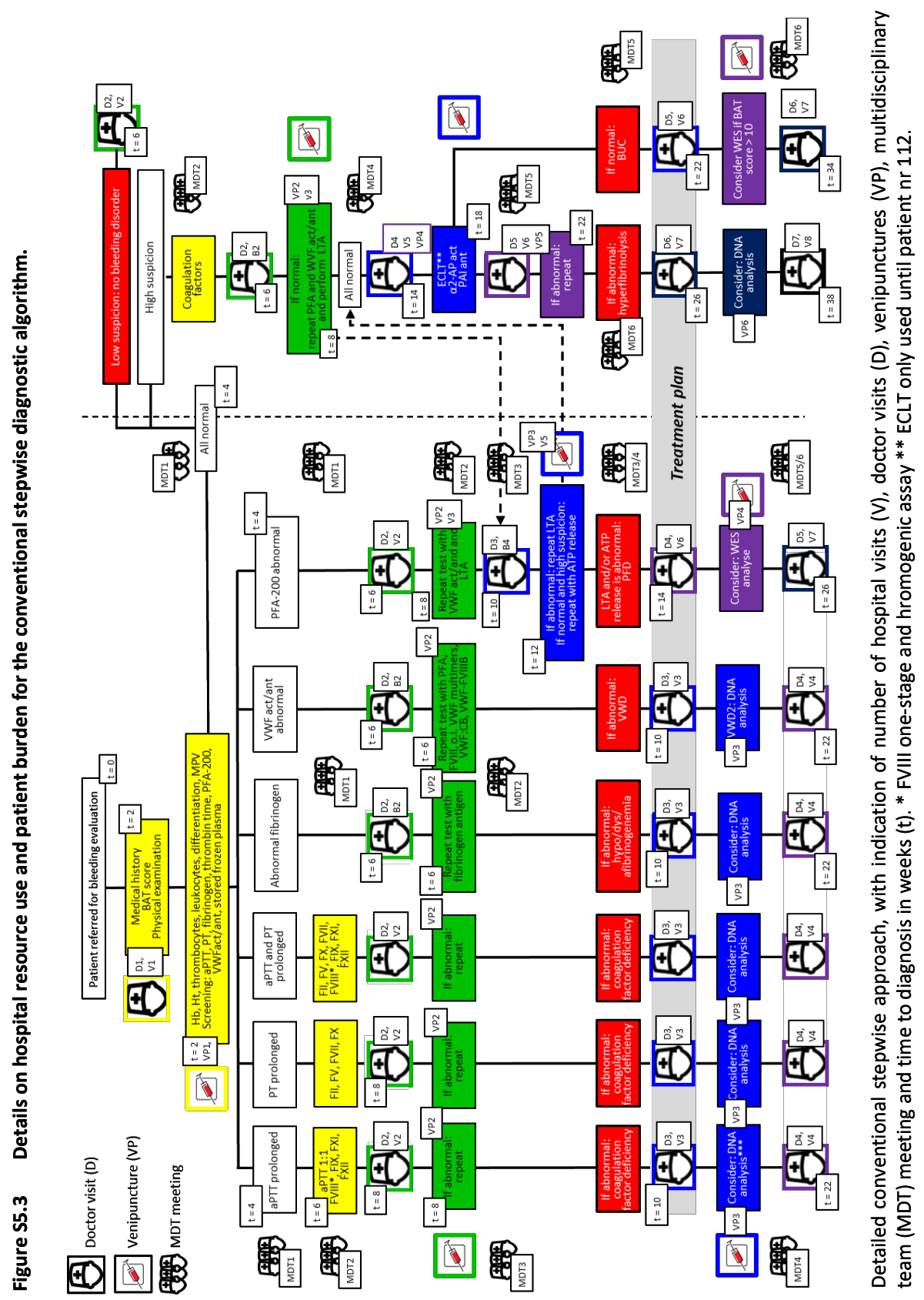




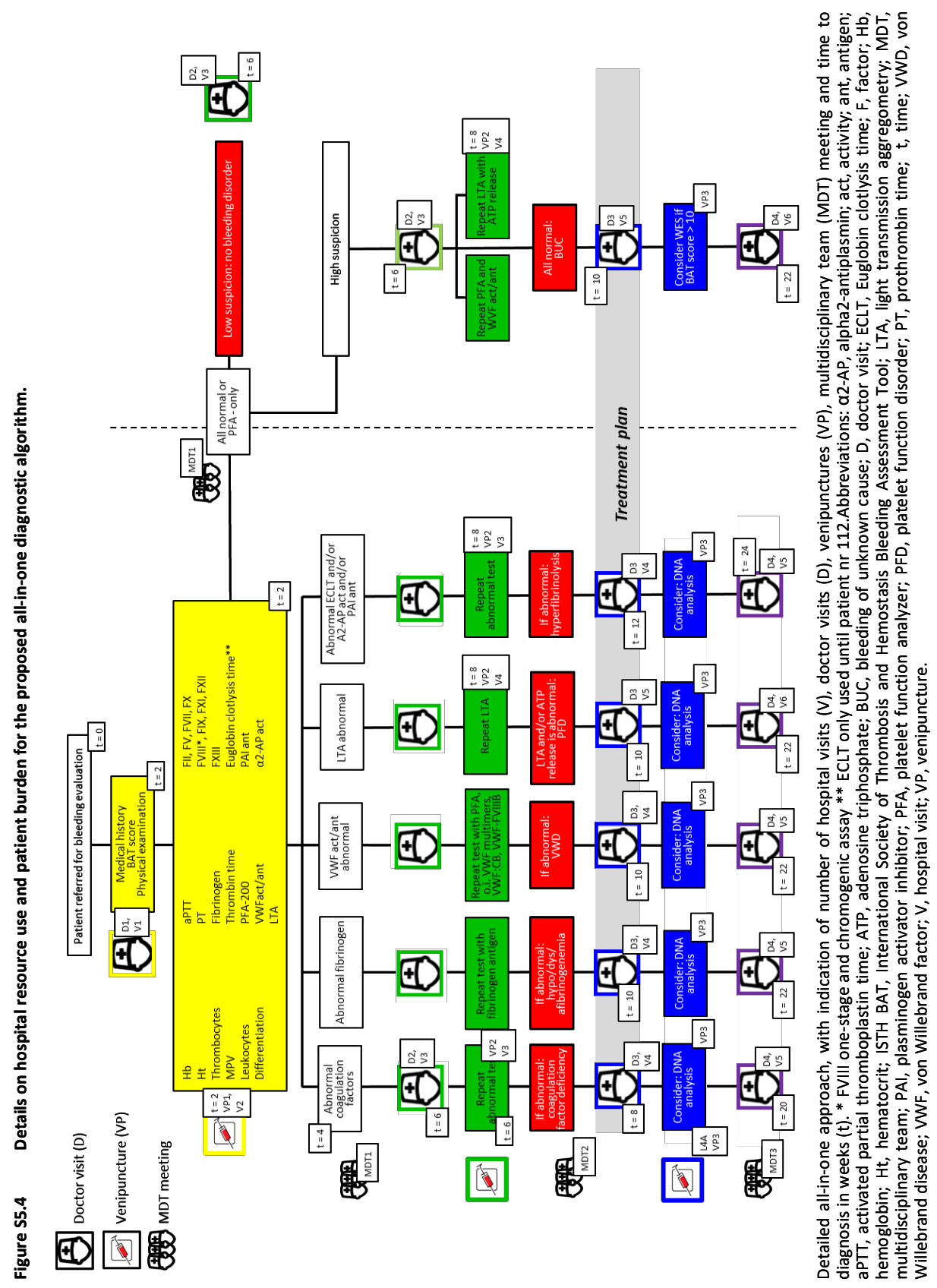




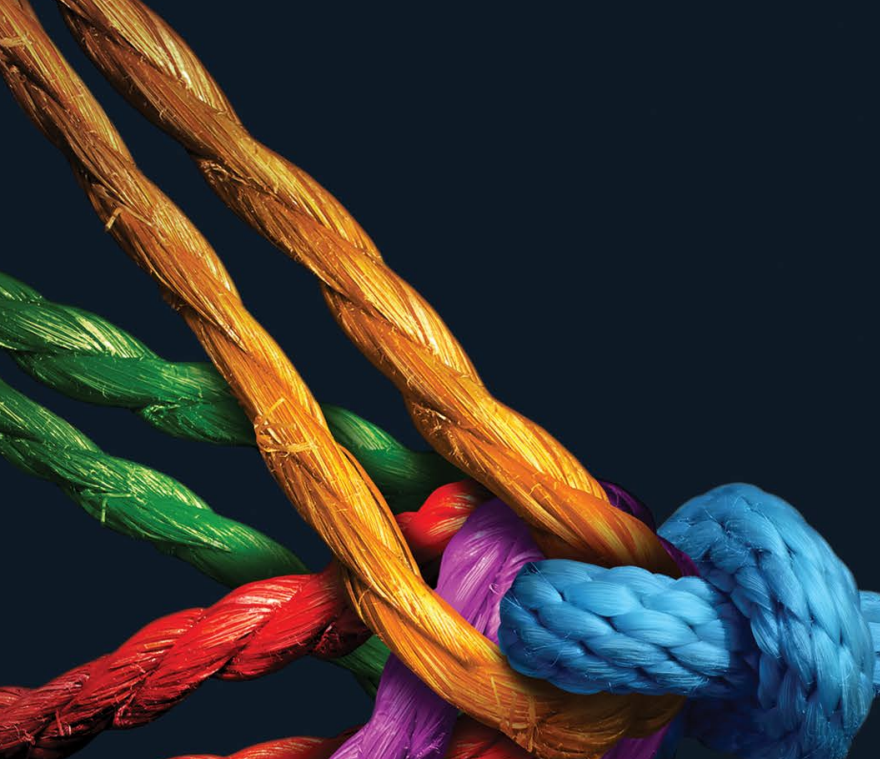




\section{PART II}

Mechanisms of bleeding in chemotherapy
induced thrombocytopenia 


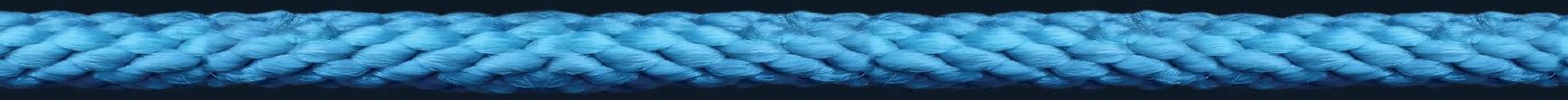




\section{CHAPTER 6}

\section{Impaired mitochondrial activity explains platelet dysfunction in thrombocytopenic cancer patients undergoing chemotherapy}

Constance C.F.M.J. Baaten, Floor C.J.I. Moenen, Yvonne M.C. Henskens, Frauke Swieringa, Rick J.H. Wetzels, René van Oerle, Harry F.G. Heijnen, Hugo ten Cate, Graham P. Holloway, Erik A.M. Beckers, Johan W.M. Heemskerk, Paola E.J. van der Meijden 


\section{Abstract}

\section{Introduction}

Severe thrombocytopenia ( $\leq 50 \times 10^{9}$ platelets/L) due to hematological malignancy and intensive chemotherapy is associated with an increased risk of clinically significant bleeding. Since the bleeding risk is not linked to the platelet count only, other hemostatic factors must be involved.

\section{Methods}

In 77 patients with acute leukemia, multiple myeloma or malignant lymphoma, who experienced chemotherapy-induced thrombocytopenia, we studied platelet function.

\section{Results}

Platelets from all patients - independent of disease or treatment type - were to a variable extent compromised in $\mathrm{Ca}^{2+}$ flux, integrin $\alpha_{11 \mathrm{~b}} \beta_{3}$ activation and P-selectin expression, when stimulated with a panel of agonists. The patients' platelets were also impaired in spreading on fibrinogen. Whereas the $\mathrm{Ca}^{2+}$ store content was unaffected, the patients' platelets showed ongoing phosphatidylserine exposure, which was not due to apoptotic caspase activity. Interestingly, mitochondrial function was markedly reduced in platelets from a representative subset of patients, as evidenced by a low mitochondrial membrane potential $(p<0.001)$ and low oxygen consumption $(p<0.05)$, while the mitochondrial content was normal. Moreover, the mitochondrial impairments coincided with elevated levels of reactive oxygen species (Spearman's rho=-0.459, $p=0.012$ ). Markedly, the impairment of platelet function only appeared after two days of chemotherapy, suggesting origination in the megakaryocytes. In patients with bone marrow recovery, platelet function improved.

\section{Conclusion}

Our findings disclose defective receptor signaling related to impaired mitochondrial bioenergetics, independent of apoptosis, in platelets from cancer patients treated with chemotherapy, explaining the low hemostatic potential of these patients. 


\section{Introduction}

Platelets are indispensable for maintaining vascular integrity and accomplishing hemostatic plug formation ${ }^{1}$. A sufficient platelet count as well as an adequate platelet function is required for prevention of bleeding. Patients with hematological malignancies, such as leukemia, multiple myeloma or malignant lymphoma, are commonly treated with combination chemotherapy, frequently followed by bone marrow transplantation. This treatment impairs proliferation of megakaryocytes and ensuing production of proplatelets. As a consequence, severe thrombocytopenia, i.e. a platelet count of $\leq 50 \times 10^{9} / \mathrm{L}$, develops in essentially all treated patients ${ }^{2}$. These patients are at high risk of bleeding, with up to $43 \%$ experiencing clinically significant bleeding (WHO grade 2 or higher), and 1\% experiencing life-threatening bleeding ${ }^{3}$. Prophylactic transfusion with platelet concentrates for preventing bleeding is given as standard care once the count drops below $10 \times 10^{9} / \mathrm{L}$ or in case of active bleeding ${ }^{2,4}$. Randomized clinical trials have indicated that the bleeding risk in this patient group is reduced by platelet transfusion, although it does not completely eliminate hemorrhagic events $\mathrm{s}^{3,5}$. Since bleeding is relatively infrequent in non-malignant thrombocytopenia ${ }^{6,7}$, it can be considered that a low platelet count is not the sole risk factor for bleeding in chemotherapy-treated patients.

Earlier studies on patients with acute myeloid leukemia, not receiving chemotherapy, have provided indications for impaired platelet function due to disease, as apparent from low platelet aggregation, reduced granule secretion and weak thromboxane $B_{2}$ production ${ }^{8-10}$. It was proposed that low expression of the $\alpha$ granule glycoprotein, P-selectin, can be used as a prognostic marker for hemorrhage ${ }^{11}$. However, bleeding in combination with thrombocytopenia is more frequently observed in cancer patients treated with chemotherapy ${ }^{12}$. The literature so far only indicates that the anthracycline daunorubicin inhibits integrin $\alpha_{11 b} \beta_{3}$ activation, aggregation and secretion of platelets upon agonist stimulation ${ }^{13,14}$. Daunorubicin and its analogue idarubicin were found to induce integrin activation and secretion in resting platelets ${ }^{15}$. However, to which extent and by which mechanism myelosuppressive chemotherapy in general affects platelet function has remained largely unclear.

In the present study, we evaluated the platelet activation processes and coagulant activity in 77 patients with hematological malignancies treated with chemotherapy. Our results point to multiple functional defects in the patients' platelets that are related to impaired mitochondrial activity, independent of classical apoptosis. In the majority of the patients, low platelet activity could be improved by platelet transfusion. 


\section{Materials and methods}

\section{Materials and methods}

See Appendix 6.A

\section{Patients and control subjects}

The study was approved by the local ethics committee (METC-11-4-097, Dutch Trial Register NL9645). All participating patients and healthy volunteers gave written informed consent according to the Helsinki declaration. Patients, reporting at the hospital, fulfilling the inclusion criteria and providing informed consent, were consecutively included in the period of November 2014 until April 2018. Eligible patients were $\geq 18$ years of age, received chemotherapy for treatment of a confirmed hematologic malignancy (acute myeloid leukemia, acute lymphoid leukemia, multiple myeloma or malignant lymphoma), and had or were expected to have thrombocytopenia (platelet count $\leq 50 \times 10^{6} / \mathrm{mL}$ ). Morning platelet counts were monitored daily as part of the routine clinical care. According to standard practice, when the morning platelet count was $<10 \times 10^{6} / \mathrm{mL}$, patients received prophylactic transfusion with one batch of platelet concentrate (leukocyte-depleted pooled buffy coat from 5 donors, median storage time: 6 days, median platelet count: $357 \times 10^{6} / \mathrm{mL}$ ). Patient exclusion criteria were: sepsis, splenomegaly, signs of active bleeding at the time of blood withdrawal, previous platelet transfusion within 3 days (excluding the presence of donor platelets), and/or use of antithrombotic medication in the past 14 days.

For clinical care, blood samples were collected before and during chemotherapeutic treatment at multiple time points: 1) before start of chemotherapy, 2) before myelosuppression, 3) during myelosuppression (platelet count $\leq 50 \times 10^{6} / \mathrm{mL}$ ), 4) during myelosuppression: before (platelet count $\leq 10 \times 10^{6} / \mathrm{mL}$ ) and $1 \mathrm{~h}$ after platelet transfusion and 5) during bone marrow recovery (platelet count $\leq 50 \times 10^{6} / \mathrm{mL}$ ). Patient blood samples were obtained via a central venous catheter, rinsed with $100 \mathrm{~mL}$ saline to remove residual traces of heparin (verified by measurement of thrombin time). Blood samples from healthy control subjects were obtained via venipuncture of the antecubital vein using a Vacutainer 21gauge needle (Becton-Dickinson Bioscience, Franklin Lakes, NJ). Blood collection was always into 3.2\% (w/v) trisodium citrate (Greiner Bio-One Vacuette, Alphen a/d Rijn, The Netherlands). For clinical care (hematological parameters), separate samples from patients were drawn into vacuette tubes containing $\mathrm{K}_{2}$-EDTA (Becton-Dickinson Bioscience, Franklin Lakes, NJ, USA). 


\section{Experimental setup}

Within the limitations of medical ethical permission, in total blood samples from 52 patients (platelet count $\leq 50 \times 10^{6} / \mathrm{mL}$ ) could be obtained during myelosuppression (study A). In all these samples, platelet responsiveness was assessed using flow cytometry. Due to the limited blood volume and the low platelet counts, a restricted number of additional analyses was carried out per sample. When sufficient sample volume was remaining, platelet function was further characterized by measuring the following platelet responses: platelet spreading, intracellular calcium signaling and phosphatidylserine (PS) exposure. To gain a deeper understanding of the underlying mechanisms of platelet dysfunction, subsequently blood samples could be obtained from 25 additional patients (platelet count $\leq 50 \times 10^{6} / \mathrm{mL}$ ) during the myelosuppression phase (study B). The samples were used to investigate apoptotic signaling (caspase activity; western blotting for caspase-mediated protein cleavage), mitochondrial respiration and structure (high-resolution respirometry, citrate synthase activity, transmission electron microscopy) or reactive oxygen species (ROS). Care was taken that for all measurements patients from the major treatment classes were represented (see figure legends).

For 36 of the patients of study A, blood samples could also be obtained at $1 \mathrm{~h}$ after transfusion with platelet concentrate. Again, platelet responsiveness was determined by flow cytometry.

\section{Statistical analysis}

Data are represented as medians with interquartile ranges. Paired data were compared using the Wilcoxon signed-rank test, otherwise the Mann-Whitney $U$ test was used. When comparing more than two groups, the Kruskal Wallis $\mathrm{H}$ test was used. $P$ values $<0.05$ were considered significant. Graphs were made using Graphpad Prism v6 (San Diego, CA, USA). Statistical analysis was performed using the SPSS Statistics 23 package (IBM, Armonk, NY, USA).

\section{Results}

\section{Variable impairment of platelet activation in cancer patients with thrombocytopenia after chemotherapy}

Blood samples were obtained from in total 77 patients, who were diagnosed with acute myeloid leukemia or acute lymphocytic leukemia ( $A M L / A L L, n=37$ ), multiple myeloma $(n=21)$, malignant lymphoma $(n=15)$ or other hematologic malignancies 
$(n=4)$. All patients experienced severe thrombocytopenia due to chemotherapy, which was stopped at median 8 days before blood sample analysis (Table 6.1). The median age of the patient group was 60 years, and $41 \%$ was female (Table 6.1). Leukocyte and platelet counts were below normal, as was the hemoglobin level. Standard coagulation parameters were determined in plasmas from 43 patients after the chemotherapy treatment. For $70 \%$ of the patients, values of aPTT, PT and thrombin time were within reference ranges (Appendix 6.B Table S6.1). Fibrinogen and VWF levels were slightly elevated, while D-dimer levels were substantially increased in the patient plasmas. On the other hand, factor VII activity levels were decreased.

Table 6.1 Characteristics and hematological parameters of patients during myelosuppression.

\begin{tabular}{|c|c|c|}
\hline Patients characteristics & \multicolumn{2}{|c|}{ Number / Value } \\
\hline Age (years) & \multicolumn{2}{|c|}{$60(60)$} \\
\hline Female/male (n) & \multicolumn{2}{|c|}{$32 / 45(20 / 32)$} \\
\hline \multicolumn{3}{|l|}{ Diagnosis (n) } \\
\hline $\mathrm{AML} / \mathrm{ALL}$ & \multicolumn{2}{|c|}{$37(25)$} \\
\hline Multiple myeloma & \multicolumn{2}{|c|}{$21(12)$} \\
\hline Lymphoma & \multicolumn{2}{|c|}{$15(13)$} \\
\hline Other & \multicolumn{2}{|c|}{$4(2)$} \\
\hline \multicolumn{3}{|l|}{ Stem cell transplantation $(\mathrm{n})$} \\
\hline Autologous & \multicolumn{2}{|c|}{$39(26)$} \\
\hline Allogeneic & \multicolumn{2}{|c|}{$11(8)$} \\
\hline Time since chemotherapy (days) & \multicolumn{2}{|c|}{$8(9)$} \\
\hline Time since stem cell transplantation (days) & \multicolumn{2}{|c|}{$8(8)$} \\
\hline Blood parameters & Value & Reference range \\
\hline Leukocyte count $\left(\times 10^{9} / \mathrm{L}\right)$ & $0.15(0.22)$ & $3.5-11.0$ \\
\hline Hemoglobin $(\mathrm{mmol} / \mathrm{l})$ & $5.7(5.7)$ & $7.5-11.0$ \\
\hline Platelet count $\left(\times 10^{9} / \mathrm{L}\right)$ & $8(7)$ & $150-400$ \\
\hline Absolute immature platelet number ( $\left.\times 10^{9} / \mathrm{L}\right)$ & $0.31(0.26)$ & \\
\hline Immature platelet fraction (\%) & $3.9(3.6)$ & $1.1-6.147$ \\
\hline
\end{tabular}

Data are for total number of patients $(n=77)$. Patient information for study $A(n=52)$ is indicated between brackets. Median values are given. AML/ALL: acute myeloid leukemia or acute lymphocytic leukemia

Treatment regimens in accordance with national guidelines varied with disease type ${ }^{16-18}$. Since these regimens consisted of multiple chemotherapeutic compounds, the distribution of the drugs was evaluated among patients with different diagnoses. Therefore, the various drugs were assigned to one of five pharmacological classes: A, antitumor antibiotics \& topo-isomerase II inhibitors; B, antimetabolites; C, alkylating agents; D, mitotic inhibitors; $E$, other (Appendix 6.B Table S6.2) ${ }^{19}$. Most patients appeared to be treated with anti-tumor antibiotics/topo-isomerase inhibitors, antimetabolites and/or alkylating agents (Appendix 6.B Table S6.3). The patients diagnosed with $A M L / A L L$ and lymphoma usually received drugs from one or more of these three classes, while the patients diagnosed with multiple myeloma only obtained alkylating agents. Of all 77 patients, 50 had undergone hematopoietic stem 
cell transplantation before inclusion, of which 39 patients received an autologous transplant and 11 an allogenic (Table 6.1). Blood samples were obtained at 8 days (median) after the last administration of chemotherapy or at 8 days (median) after stem cell transplantation.

Responsiveness of washed platelets was determined by flow cytometry, using a platelet count of $10 \times 10^{6} / \mathrm{mL}$, for 52 patients and 27 healthy control subjects. In the absence of agonists, surface activation markers were low for both patient and control platelets. After stimulation with ADP (P2Y $\mathrm{Y}_{1 / 12}$ agonist), CRP-XL (GPVI agonist) or thrombin (PAR1/4 agonist) at maximal doses, integrin $\alpha_{11 b} \beta_{3}$ activation (Figure 6.1A) and P-selectin expression (Figure 6.1B) of the patients' platelets were reduced to a variable extent, when compared to the controls, irrespective of the agonist used.

Detailed analysis indicated that the overall platelet responsiveness (median: 36.8\%, IQR: $29.7-46.7 \%)$, defined as the average fraction of platelets positive for integrin activation and P-selectin expression for the three agonists: (i) was not different between diagnoses, i.e. AML/ALL, multiple myeloma, lymphoma and other hematological malignancies (Kruskal Wallis $\mathrm{H}$ test, $p=0.192$ ); (ii) was not affected by stem cell transplantation, i.e. no transplant, autologous or allogenic stem cell transplantation (Kruskal Wallis $\mathrm{H}$ test, $p=0.640$ ); (iii) was similar for the four major treatment classes, i.e. $A+B, A+B+C, B+C, C$ (Kruskal Wallis $\mathrm{H}$ test, $p=0.512$; Appendix 6.B Figure S6.1); and (iv) did not correlate with the whole blood platelet count (Spearman's $r h o=0.175, p=0.239$ ). Together, this suggested that the variability in platelet responsiveness among patients was not directly linked to the disorder, treatment type or number of (residual) circulating platelets. Additional functional analyses were performed with platelets, always from patients in the major treatment classes.

For 36 of the patients, blood samples could be obtained before and $1 \mathrm{~h}$ after platelet transfusion. As expected, platelet count increased after transfusion (Appendix 6.B Table S6.4). The clinical efficacy of transfusion was evaluated from the corrected count increment $[\mathrm{CCl}$ : (platelet count increment $\mathrm{x}$ body surface area)/(number of transfused platelets $\left.\times 10^{11}\right)^{20}$. This was adequate for $96 \%$ of the patients, as indicated by a $\mathrm{CCl}$ value of $>7.5$ (median: 14.8, IQR: 11.3-18.0).

Flow cytometric analysis of integrin activation and P-selectin expression demonstrated that at $1 \mathrm{~h}$ after transfusion, platelet responsiveness was improved for most patients (Appendix 6.B Figure S6.2). Whenever possible, platelets were also isolated from the remainder of the transfusion concentrates. It appeared that the activity of the circulating platelets after transfusion approached that of the platelets of the concentrates, when triggered with thrombin or CRP-XL. However, the responsiveness to ADP of the circulating platelets after transfusion was higher than in 
concentrates (integrin $\alpha_{11 b} \beta_{3}$ activation, $p=0.002$ ). The improved platelet responses after transfusion underlined the low responsiveness of the autologous platelets after chemotherapy.

Figure 6.1 Variable impairment of integrin $\alpha_{11 \mathrm{~b}} \beta_{3}$ activation and P-selectin expression in stimulated platelets from cancer patients with thrombocytopenia after chemotherapy.
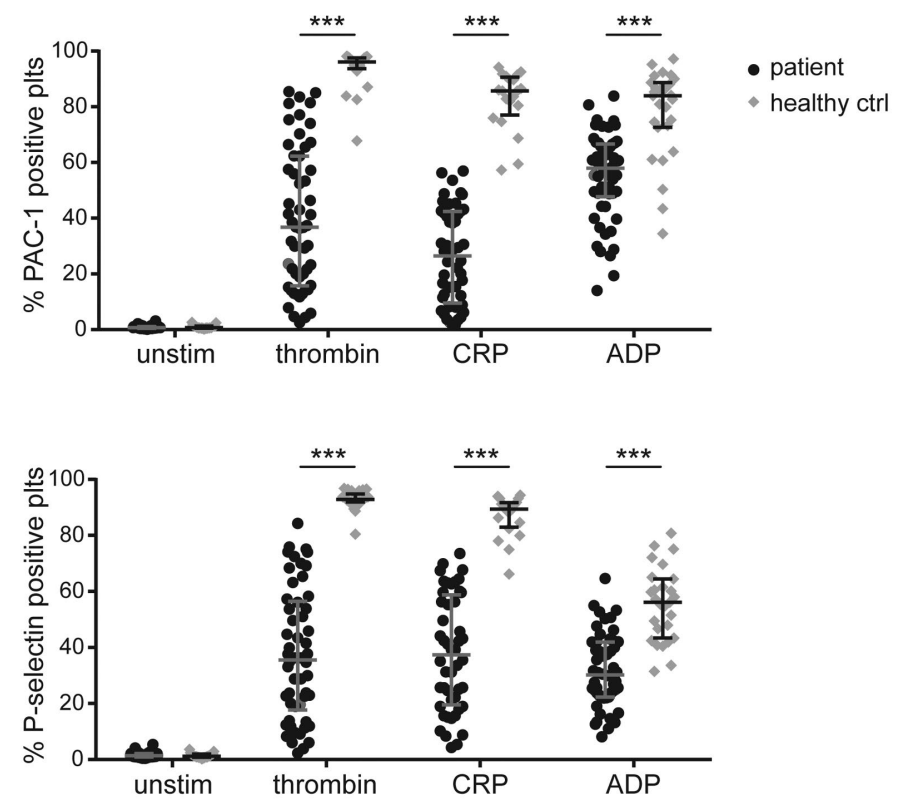

Washed platelets $\left(10 \times 10^{9} / \mathrm{L}\right)$ from healthy control subjects (healthy ctrl) and thrombocytopenic patients receiving chemotherapy were activated with thrombin $(4 \mathrm{nM}), \mathrm{CRP}-\mathrm{XL}(10 \mu \mathrm{g} / \mathrm{mL})$ or $2 \mathrm{MeS}-\mathrm{ADP}(1 \mathrm{nM})$ in the presence of $2 \mathrm{mM} \mathrm{CaCl}_{2}$. After 15 min activation, integrin $\alpha_{\| 1 b} \beta_{3}$ activation $(A)$ and $P$-selectin expression (B) were measured by flow cytometry using PAC-1 and anti-P-selectin antibody, respectively. Medians with IQR; datafrom 52 patients ( $25 \mathrm{AML} / \mathrm{ALL}, 12$ multiple myeloma, 13 lymphoma, twoother), 27 healthy controls, $* * * p<0.001$. CRP: collagen-related peptide; ADP: adenosine diphosphate.

\section{Impaired platelet responsiveness during myelosuppression}

To determine whether the reduced platelet responsiveness was linked to the treatment phase, flow cytometric analysis of platelet responsiveness was performed during the decreasing period of platelet count $\left(50-11 \times 10^{6} / \mathrm{mL}\right.$ and $\left.\leq 10 \times 10^{6} / \mathrm{mL}\right)$ and the recovery of platelet count $\left(11-50 \times 10^{6} / \mathrm{mL}\right)$. The latter was defined as a sustained increase in platelet count (observed for patient care), independent of platelet transfusion. Of the eight patients included in this category, three had received an autologous transplant and one patient an allogeneic stem cell transplant, prior to recovery. In the decreasing period, integrin activation and P-selectin expression following stimulation with thrombin 
or CRP-XL were comparable in patients with platelet counts in the range of $50-11 \times 10^{6} / \mathrm{mL}$ and $\leq 10 \times 10^{6} / \mathrm{mL}$ (Figure 6.2).

Figure 6.2 Impaired platelet responsiveness in relation to phase of treatment and/or recovery.
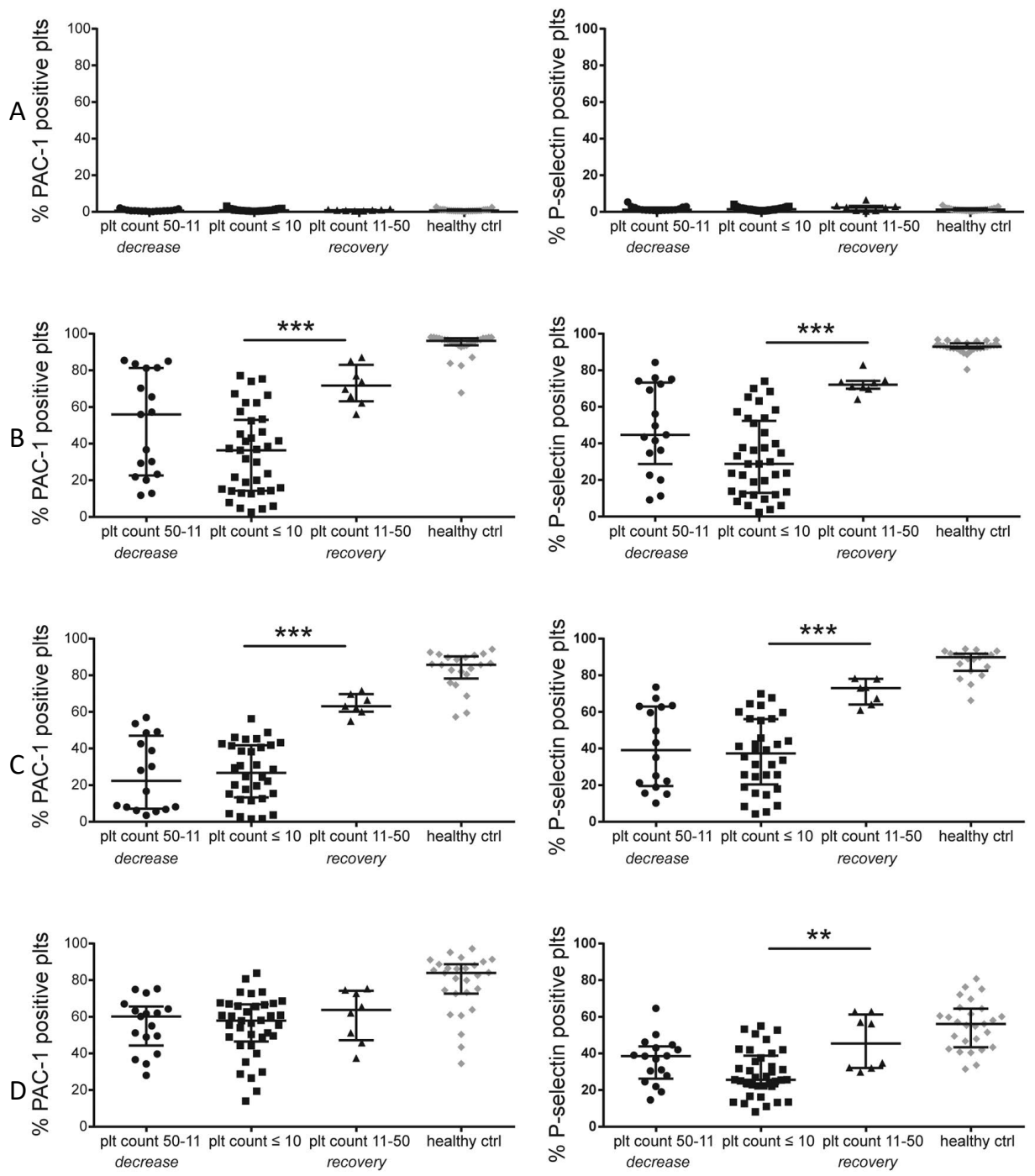

Platelet integrin $\alpha \mathrm{llb} \beta 3$ activation and P-selectin expression were measured (see Figure 6.1). Patients $(n=52)$ were divided into two categories: (i) decreasing platelet count 50-11 x 10\% $/ \mathrm{mL}(\mathrm{n}=15$ ) and (ii) decreasing platelet count $\leq 10 \times 10^{6} / \mathrm{mL}(\mathrm{n}=37)$. Furthermore, from a subset of patients a sample could be collected when the platelet count increased independently of platelet transfusion (iii): $11-5 \times 10^{6} / \mathrm{mL}(\mathrm{n}=8)$ ). Data are expressed as \% of platelets positive for PAC- 1 or anti-P-selectin staining in the absence of stimulation (A), or after stimulation with thrombin (B), CRP-XL (C) or 2MeS-ADP (D). Medians with IQR for patients and healthy controls $(\mathrm{n}=27) ;{ }^{* *} p<0.01$ and $* * * p<0.001$. 
In contrast, platelet responsiveness to thrombin and CRP-XL significantly improved in case of count recovery $(p<0.001)$. For stimulation with ADP, these differences were less pronounced, with only P-selectin expression increased during count recovery. These results indicated that platelet count alone is not a good marker of platelet activity.

For five patients ( $1 \mathrm{AML}, 3$ multiple myeloma, 1 lymphoma), blood samples could also be analyzed at an earlier time point, i.e. after the stop of chemotherapy, but before severe thrombocytopenia occurred. Remarkably, in all these samples, platelet function was within normal range for the three agonists (integrin activation 69-86\%, P-selectin expression 49-85\%). Furthermore, in vitro treatment of control blood with clinically relevant concentrations of cytarabine and/or melphalan did not affect platelet reactivity (Appendix 6.B Figure S6.3A-B). These results argue against a direct effect of the chemotherapeutics on the platelet activation properties.

\section{Impaired platelet spreading and Ca2+ signaling of platelets after chemotherapy treatment}

To further characterize the patient platelets, they were allowed to adhere and spread for $10 \mathrm{~min}$ on a fibrinogen surface, interacting with platelet integrin $\alpha_{11 b} \beta_{3}$. The observed morphology of the cells was divided into three stages: 1) formation of filopodia, 2) formation of lamellipodia, and 3) full spreading. Most of the platelets from control subjects were in stages $2-3$, while the patient platelets predominantly stayed in stage 1 (forming filopodia only), with few platelets being fully spread (Figure 6.3A). The patients' platelets displayed a slightly decreased expression of GPIba and GPVI, but not in integrin $\beta_{3}$ expression (data not shown). This suggested a diminished integrin activity and outside-in signaling in the patient platelets.

We further examined agonist-induced $\mathrm{Ca}^{2+}$ signaling after loading the platelets with Fluo-4. Stimulation with thrombin or CRP-XL induced only a small rise in $\left[\mathrm{Ca}^{2+}\right]_{\mathrm{i}}$ in patient platelets, when compared to control platelets (Figure 3.6.B-C). On the other hand, the $\left[\mathrm{Ca}^{2+}\right]_{i}$ rise induced by thapsigargin (an inhibitor of endoplasmic reticulum $\mathrm{Ca}^{2+}$-ATPases), as a measure of $\mathrm{Ca}^{2+}$ store content ${ }^{21}$, was similar for patient and control platelets. Together, this pointed to a defective agonist-induced $\mathrm{Ca}^{2+}$ signaling machinery, independently of receptor type (i.e., PAR1/4 or GPVI receptors). 
Figure 6.3 Impaired platelet spreading and $\mathrm{Ca}^{2+}$ signaling of platelets from patients.

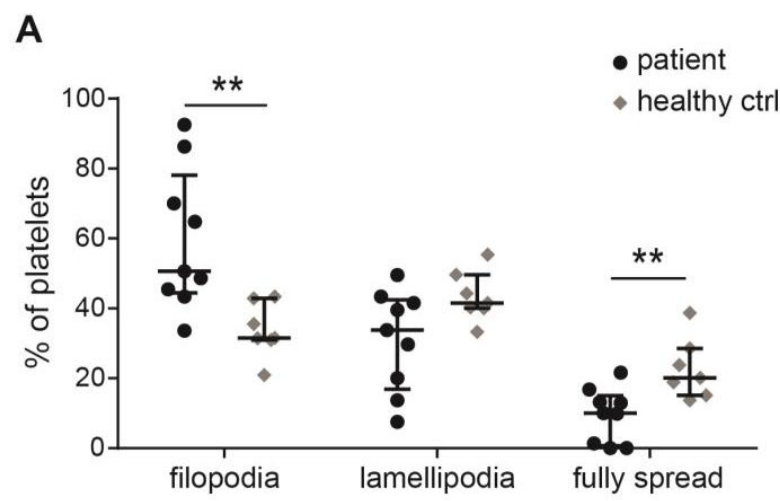

$\mathbf{B}$
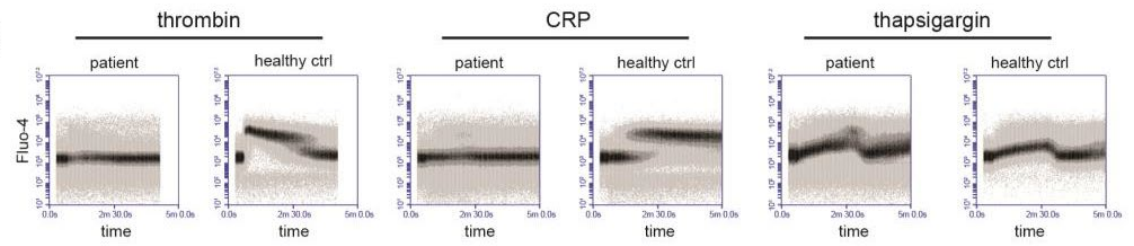

C

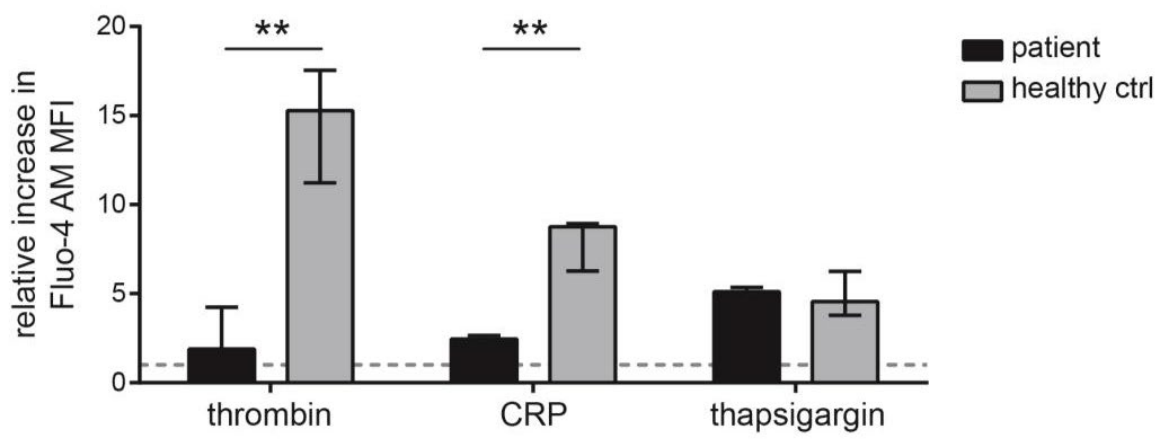

(A) Platelets from patients or healthy controls were allowed to spread on a fibrinogen surface for $10 \mathrm{~min}$, after which microscopic images were captured. Spreading state per platelet was classified in three stages based on morphology: (i) filopodia, (ii) lamellipodia, or (iii) fully spread. Percentages of platelets per category are shown. Medians (with IQR) for 9 patients, 7 control subjects. (B, C) Fluo-4-loaded platelets from patients $(n=7)$ and controls $(n=5)$ were stimulated with thrombin $(4 \mathrm{nM}), \mathrm{CRP}-\mathrm{XL}(10 \mu \mathrm{g} / \mathrm{mL})$ or thapsigargin $(0.5 \mu \mathrm{M})$ in the presence of $2 \mathrm{mM} \mathrm{CaCl}$. Changes in Fluo-4 fluorescence were measured in time by flow cytometry. (B) Representative Fluo-4 traces in time. (C) Relative increases in cytosolic Ca2+. Medians with IQR, ${ }^{* *} p<0.01$. Overall platelet responsiveness of the patients was $31.5-57.9 \%$ (IQR). 


\section{Impaired mitochondrial bioenergetics but no apoptosis in platelets after chemotherapy}

Given the cytotoxicity of chemotherapeutic compounds, we evaluated if patient platelets showed characteristics of apoptosis, since this process is known to lead to dysfunctional signaling 22 . As a marker of apoptosis, PS exposure was determined by FITC-annexin A5 binding. In contrast to control platelets, the patient platelets were prone to expose PS upon short-term storage without external stimuli (Figure 6.4A). Upon stimulation with the $\mathrm{BH} 3$ mimetic $\mathrm{ABT}-737$, triggering the intrinsic pathway of apoptosis $^{22}$, PS exposure was initially accelerated in the patient platelets, when compared to control platelets (Figure 6.4B). As expected, preincubation with the pancaspase inhibitor Q-VD-OPh fully inhibited the PS exposure triggered by ABT-737. However, Q-VD-OPh failed to affect the storage-dependent PS exposure (Figure 6.4C). Furthermore, whereas ABT-737 stimulation resulted in high caspase-3 activity, no such activity could be detected during storage (Figure 6.4D). Additional confirmation for the absence of apoptotic signaling was obtained by assessing the caspasedependent cleavage of the integrin-binding protein, kindlin- $3^{23}$. Western blot analysis indicated that, in platelets from control subjects, ABT-737 treatment induced full cleavage of kindlin-3, which was prevented by Q-VD-OPh (Figure 6.4E). In the patient platelets (with confirmed functional impairment of integrin activation and P-selectin expression), however, no kindlin-3 cleavage could be detected in the absence of ABT-737.

Platelet activation is known to rely on mitochondrial activity for sufficient ATP production ${ }^{24}$. Given that mitochondrial impairment can lead to PS exposure ${ }^{25,26}$, we assessed the activity of mitochondria in several ways. As part of the initial characterization of the patient platelets, the mitochondrial membrane potential was assessed by staining with TMRE. Whereas control platelets displayed high TMRE fluorescence, the patient platelets showed much less fluorescence intensity (Figure $6.5 \mathrm{~A})$. This suggested a depolarization of the platelet mitochondria, which was independent of diagnosis or treatment class (Kruskal-Wallis $\mathrm{H}$ test, $p=0.656$ and $p=0.126$, respectively). The low TMRE fluorescence correlated well to the reduced platelet responsiveness (Spearman's $r h o=0.569, p=0.001$ ). However, cyclosporin Ainduced inhibition of mitochondrial permeability pore formation did not affect PS exposure (data not shown).

We subsequently assessed platelet mitochondrial activity, by measuring the mitochondrial respiration using high-resolution respirometry ${ }^{27}$. With saturating amounts of complex I-II substrates of the oxidative phosphorylation (OXPHOS) chain, i.e. pyruvate, malate, ADP, glutamate and succinate, the maximal ADP-supported respiration of mitochondria was significantly lower in platelets from patients than 
from controls (Figure 6.5B). To exclude that the mitochondrial content was altered, we measured the citrate synthase activity ${ }^{28}$. However, this was unchanged in the patients' platelets (Figure 6.5C). Transmission electron microscopic images were also recorded, and these did not reveal structural abnormalities of the mitochondria (data not shown).

Figure 6.4 Increased PS exposure in platelets from patients receiving chemotherapy in the absence of apoptosis.

A

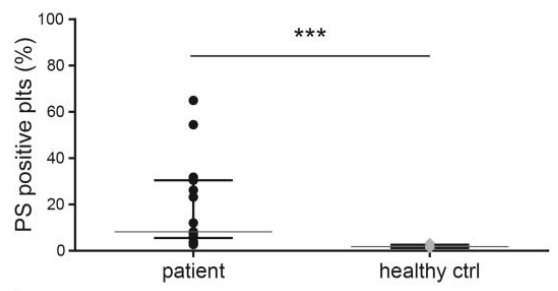

C

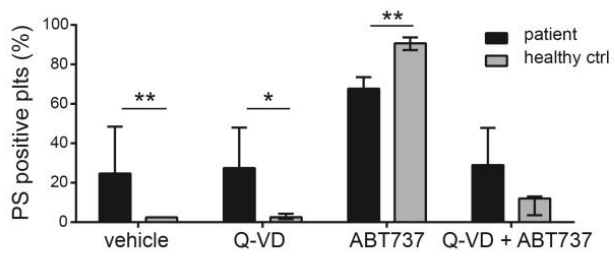

E

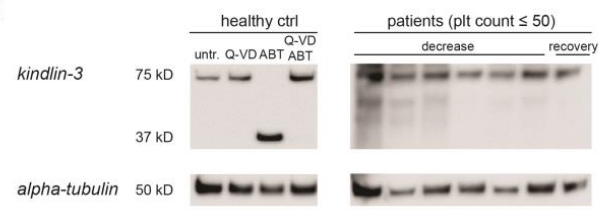

B

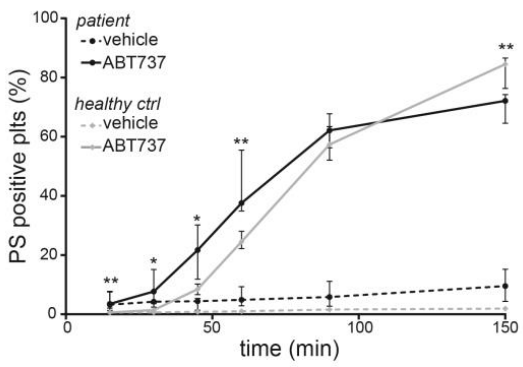

D

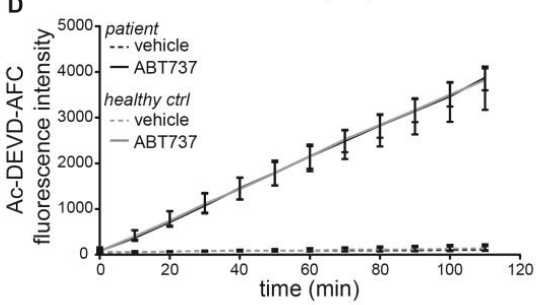

Isolated platelets from patients and controls were incubated at $37^{\circ} \mathrm{C}$ for $90 \mathrm{~min}$, and stained for PS exposure with FITC-annexin A5. (A) Percentages of PS-exposing platelets, (patients $n=15$, controls $n=12$ ). (B) PS exposure measured after indicated times with vehicle or $5 \mu \mathrm{M} \mathrm{ABT-737}$ to induce apoptosis, $(n=6-9)$. Platelets $\left(10 \times 10^{6} / \mathrm{mL}\right)$ from patients or controls were pretreated with caspase inhibitor Q-VD-OPh $(10 \mu \mathrm{M})$, as indicated, and then stimulated with $\mathrm{ABT}-737(5 \mu \mathrm{M})$ or vehicle. (C) Fractions of platelets with PS exposure, measured with FITC-annexin A5, ( $n=8)$. (D) Caspase-3 activity determined with a fluorometric assay, $(n=4)$. (E) Absence of caspase-dependent kindlin-3 cleavage in western blots from patient platelets. Control platelets were stimulated with ABT-737 with(out) Q-VD-OPh pretreatment; patient platelets were analyzed during the decreasing and recovery phases of platelet count, $(n=7)$. Overall platelet responsiveness of the patients was $30.5-48.4 \%$ (IQR). Medians with IQR, ${ }^{*} p<0.05,{ }^{* *} p<0.01$ and ${ }^{* * *} p<0.001$. 
Figure 6.5 Impaired mitochondrial bioenergetics in patient platelets.

A

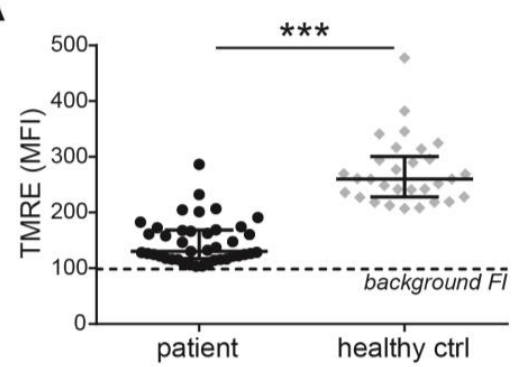

B
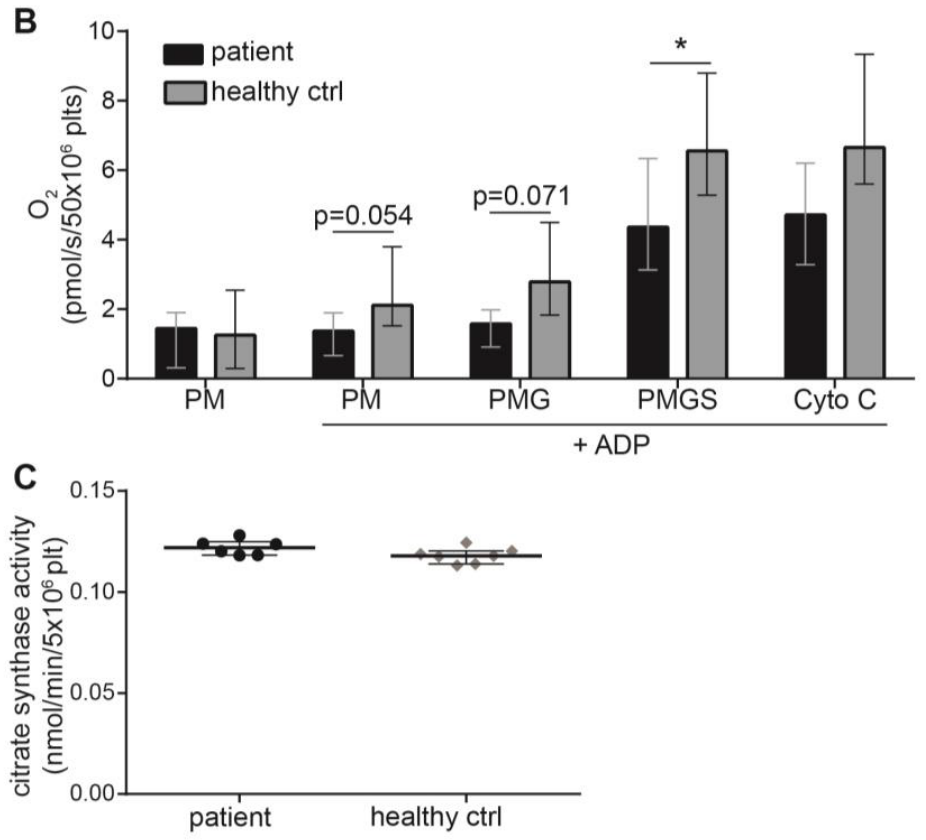

(A) Initial screening of TMRE staining of washed platelets from patients. To assess the mitochondrial membrane potential, platelets were stained with TMRE and subsequently, analyzed by flow cytometry. Shown are mean fluorescence intensities of TMRE. ( $n=39$ : treatment classes: $A+B: n=10 ; A+B+C: n=5 ; B+C$ : $n=7 ; C: n=13)$ and healthy controls $(n=27)$. (B) High resolution respirometry to measure mitochondrial respiration in washed platelets from additionally included patients $(n=7)$ and controls $(n=9)$. Depicted is oxygen consumption due to sequential addition of saturating amounts of pyruvate (P), malate (M), ADP, glutamate (G), succinate (S) and cytochrome $C$ (Cyto C). (C) Citrate synthase activity in washed platelets from patients $(n=6)$ and controls $(n=7)$ to assess mitochondrial content. Medians with IQR, ${ }^{*} p<0.05$, $* * * p<0.001$. Overall platelet responsiveness of the patients was $28.7-46.9 \%$ (IQR).

Chemotherapeutics like anthracycline analogues can cause (cardio)myopathy and neuropathy by inducing mitochondrial damage, a process mediated by oxidative stress $^{29,30}$. To determine whether a similar process is operative in the platelet lineage, 
activation markers, mitochondrial function (TMRE) and ROS levels were measured in platelets from seven patients before and after two days after chemotherapy. Additional blood samples were analyzed, when severe thrombocytopenia occurred (median 10 days after last treatment; median count $11 \times 10^{9} / \mathrm{L}$ ). Before the start of chemotherapy, platelet reactivity in these patients was comparable to that of healthy controls (Figure 6.6A-B). After two days of therapy, platelet count was slighter lowered (median decrease: $15 \times 10^{9} / \mathrm{L}$, IQR: 12.5-24.5), but platelet reactivity was not significantly changed. In contrast, reactivity in response to all agonists markedly lowered, when the patients became thrombocytopenic. Similarly, TMRE fluorescence only decreased in the latter case (Figure 6.6C), which only then was accompanied by a higher ROS production (Figure 6.6D). The reduction in TMRE fluorescence correlated with the production of ROS (Spearman's rho $=-0.459, p=0.012$ ). Treatment of control platelets in vitro with chemotherapeutics did neither affect the mitochondrial membrane potential nor the ROS production (Appendix 6.B Figure S6.3C-D). Together, these results strongly suggest that the mitochondrial dysfunction is not caused by a direct effect of chemotherapeutics on platelets, but by affecting the platelet precursor cells, the megakaryocytes.

\section{Discussion}

In this paper, we provide novel evidence that the platelets from thrombocytopenic patients, suffering from hematological malignancies and treated with myeloablative chemotherapy, are dysfunctional in multiple aspects. We found that key agonistinduced responses of the patients' platelets, such as integrin activation, secretion and $\mathrm{Ca}^{2+}$ fluxes are impaired, remarkably at a variable extent. Furthermore, the platelets from almost all patients showed agonist-independent exposure of PS upon storage, which was not linked to apoptotic caspase activity; such in contrast to the platelets from healthy subjects, not displaying PS exposure. In the patients' platelets, the defective activation could be linked to an impaired mitochondrial membrane potential and a decreased mitochondrial respiratory activity.

The impaired platelet responsiveness after myeloablative chemotherapy (median of 8 days) was only weakly correlated to the whole blood platelet count, indicating that the extent of thrombocytopenia as such was not a main factor in the dysfunction. In agreement with this conclusion, in patients with a recovering platelet count after transplantation, the functionality of the platelets improved towards normal. Detailed analysis indicated that neither disease type nor chemotherapy regimen could explain the inter-patient variation in platelet responsiveness. This points to other factors 
determining the severity of dysfunction, such as a different sensitivity of megakaryocytes in the bone marrow to the previous chemotherapy treatment. As the sensitivity of megakaryocytic precursor cells to chemotherapeutics is known to vary ${ }^{31}$, the extent of platelet dysfunction might be a combined result of the sensitivity of the precise drugs administered and their dosing.

Figure 6.6 Decreased responsiveness of patient platelets is accompanied by mitochondrial membrane depolarization and ROS production.
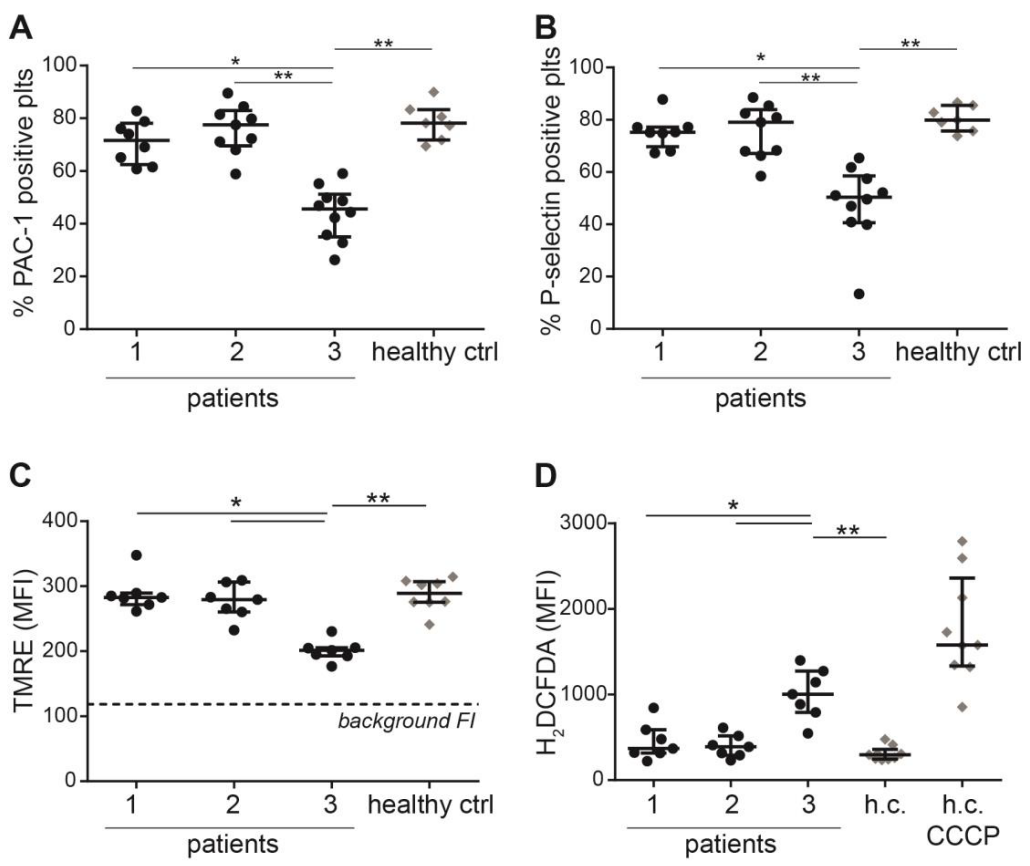

Platelets $\left(10 \times 10^{6} / \mathrm{mL}\right)$ were isolated from healthy controls (healthy ctrl) and from patients at three time points, namely 1: directly before the start of chemotherapy, 2: at 2 days of chemotherapy and, 3: upon severe thrombocytopenia (count $\leq 50 \times 10^{9} / \mathrm{L}$ ). Washed platelets were activated with thrombin $(4 \mathrm{nM}), \mathrm{CRP}$ $\mathrm{XL}(10 \mu \mathrm{g} / \mathrm{mL})$ or $2 \mathrm{MeS}-\mathrm{ADP}(1 \mu \mathrm{M})$ in the presence of $2 \mathrm{mM} \mathrm{CaCl}$. After 15 min activation, integrin $\alpha_{\| 1 \mathrm{~b}} \beta_{3}$ activation (A) and P-selectin expression (B) were measured by flow cytometry using labeled PAC-1 and antiP-selectin antibody, respectively. Depicted is mean platelet responsiveness to thrombin, CRP-XL and ADP. Platelet samples were loaded with TMRE (C) to assess mitochondrial membrane potentials, indicative of mitochondrial function, or with H2DCFDA (D) to measure ROS levels. Platelets from healthy controls were treated with CCCP as a positive control (h.c. CCCP). Medians with interquartile ranges (IQR); $n=7-10$ (patients) and $\mathrm{n}=7$ (healthy controls), ${ }^{*} p<0.05,{ }^{* *} p<0.01$ and ${ }^{* * *} p<0.001$. 
The dysfunction of platelets identified in this patient group markedly differs from the so-called 'exhausted' platelets, which have been described for patients with solid tumors ${ }^{32}$. Exhausted platelets were characterized by a high integrin activation and Pselectin expression in the absence of stimulating agents, and a reduced increase in the parameters after agonist stimulation. These changes might point to platelet activation in vivo, resulting in a secondary loss of function ${ }^{33}$. Given that in the present patient group P-selectin expression and integrin activation were low without stimulation, there is no evidence for in vivo platelet activation linked to chemotherapy treatment. On the other hand, the patients' platelets showed an tendency to expose PS, which is compatible with an apoptotic process, as apoptotic platelets are known to be defective in aggregation and secretion ${ }^{22}$. However, ongoing apoptotic signaling could be excluded, since: (i) treatment with the pan-caspase inhibitor Q-VD-OPh did not prevent PS exposure, (ii) measurable caspase-3 activity was absent, and (iii) caspasedependent cleavage of kindlin-3 could not be detected.

Platelets rely on mitochondrial ATP production, in particular upon activation when their energy demand increases ${ }^{24}$. While the mitochondrial content and ultrastructure appeared normal in the patients' platelets, we noticed a marked reduction of the platelet mitochondrial membrane potential and the mitochondrial oxidative phosphorylation. Other authors have shown that anti-tumor antibiotics (anthracyclines), an important class of chemotherapeutic agents used to treat hematological malignancies, induce cardiotoxicity and muscle weakness due to impairment of mitochondrial function via increased production of $\operatorname{ROS}^{29,34,35}$. In cardiac cells, the accumulation of iron inside the mitochondria may contribute to the production of $\operatorname{ROS}^{36}$. Furthermore, the mitochondrial activity in myocardial and hepatic cells is known to be impaired by the chemotherapeutics cyclophosphamide and carmustine $(\mathrm{BCNU})^{37-39}$. Our results suggest that a similar mechanism of ROSlinked mitochondrial dysfunction is operative in the platelet precursor cells, as deduced from the strong correlation (at >2 days after treatment) between mitochondrial dysfunction and elevated ROS levels. The fact that platelet activation induced by strong agonists (CRP-XL, thrombin) was more affected than platelet activation by ADP suggests a relatively larger role of mitochondrial ATP production upon stimulation with stronger agonists ${ }^{40}$. The slight decrease in GPVI (and GPIb $\alpha$ ) receptor levels might contribute to the lower responsiveness of platelets, although this can also be the consequence of receptor shedding induced by ROS and mitochondrial stress ${ }^{41}$.

Together, our findings suggest that ROS-induced dysfunction in the mitochondria (before the production of platelets) impairs platelet activity and induces PS exposure, thus leading to a shortened platelet lifetime. This conclusion is supported by a recent 
study in mice, developing thrombocytopenia after 5-fluororacil treatment. In these animals low-level laser therapy was found to increase the mitochondrial activity of megakaryocytes, resulting in a normalization of hemostasis ${ }^{42}$. Other possible strategies to improve mitochondrial function after chemotherapy are administration of antioxidants to reduce ROS, considering that patients with hematological malignancies have low levels of vitamin $C^{30,43}$. Alternatively, treatment with metformin to improve the mitochondrial energy metabolism could be beneficial ${ }^{30}$.

Due to ethical limitations, we could not assess whether the platelet dysfunction after chemotherapeutic treatment was linked to abnormal (pro)platelet formation from megakaryocytes in the bone marrow. The available literature yet suggests that the progenitor cells are more vulnerable towards chemotherapy than matured megakaryocytes ${ }^{31}$. In patients who received chemotherapy and had not yet developed thrombocytopenia, we observed a normal platelet activity comparable to that before treatment had started. Furthermore, in vitro treatment of whole blood from healthy controls with cytarabine and/or melphalan did neither affect platelet reactivity nor mitochondrial function. This agrees with an indirect drug effect on the megakaryocytes or precursor cells, rather than a direct effect on the circulating platelets.

With regards to the coagulant state, the reduced level of factor VII found in combination with high circulating D-dimers in the patients' plasmas is suggestive for an mild ongoing state of tissue factor-triggered coagulation ${ }^{44}$. However, the data do not provide evidence for appreciable consumption of other coagulation factors. Given that factor VII has a short half-life in blood ${ }^{45}$, it will be the first coagulation factor to decline upon ongoing coagulation. Chemotherapy can induce endothelial cell activation and upregulate tissue factor levels ${ }^{46}$, which also can explain the elevated VWF levels in patients. The increased bleeding tendency is most likely the result of the impaired platelet function, without compensation by a higher coagulant activity. Moreover, although the relative number of PS positive platelets is high, given their fast clearance from circulation, it is unlikely that this platelet population would significantly compensate for primary hemostasis.

The present study has several limitations. Given that the number of isolated platelets was limited due to the severe thrombocytopenia, only a restricted subset of measurements could be performed per patient blood sample, with as a consequence that different patient samples needed to be used for some of the measurements. Furthermore, platelet samples were analyzed from patients with different disease types (AML/ALL, multiple myeloma and malignant lymphoma) after receiving chemotherapy in distinct treatment regimens. Here we like to stress that a reduced platelet function was detected in all patient groups and all therapeutic regimens. 
Current guidelines for prophylactic transfusion during myelosuppression are based on platelet count only. Our novel findings indicate that, next to the platelet count, also the activity of circulating platelets needs to be considered for an optimal control of hemostasis. This work thus urges for an inclusion of platelet function assays for the prediction of bleeding in this patient group. 


\section{References}

1. Versteeg HH, Heemskerk JW, Levi M, Reitsma PH. New fundamentals in hemostasis. Physiol Rev. 2013;93:327-58.

2. Apelseth TO, Hervig T, Bruserud $\varnothing$. Current practice and future directions for optimization of platelet transfusions in patients with severe therapy-induced cytopenia. Blood Rev. 2011;25:113-22.

3. Stanworth SJ, Estcourt LJ, Powter G, Kahan BC, Dyer C, Choo L, et al. A no-prophylaxis platelettransfusion strategy for hematologic cancers. N Engl J Med. 2013;368:1771-80.

4. Estcourt L, Stanworth SJ, Doree C, Hopewell S, Murphy MF, Tinmouth A, et al. Prophylactic platelet transfusion for prevention of bleeding in patients with haematological disorders after chemotherapy and stem cell transplantation. Cochrane Database Syst Rev. 2012;16(5):CD004269.

5. Wandt H, Schaefer-Eckart K, Wendeling K, Pilz B, Wilhelm M, Thalheimer M, et al. Therapeutic platelet transfusion versus routine prophylactic transfusion in patients with haematological malignancies: an open-label, multicentre, randomised study. Lancet. 2012;380(9850):1309-16.

6. Friedmann AM, Sengul H, Lehmann H, Schwartz C, Goodman S. Do basic laboratory tests or clinical observations predict bleeding in thrombocytopenic oncology patients? A reevaluation of prophylactic platelet transfusions. Transfus Med Rev. 2002;16(1):34-45.

7. Slichter SJ. Relationship between platelet count and bleeding risk in thrombocytopenic patients. Transfus Med Rev. 2004;18(3):153-67.

8. Cowan DH, Graham RC, Baunach D. The platelet defect in leukemia. J Clin Invest. 1975;56:188-200.

9. Woodcock BE, Cooper PC, Brown PR, Pickering C, Winfield DA, Preston FE. The platelet defect in acute myeloid leukaemia. J Clin Pathol. 1984;37:1339-42.

10. Leinoe EB, Hoffmann $\mathrm{MH}$, Kjaersgaard E, Johnsen HE. Multiple platelet defects identified by flow cytometry at diagnosis in acute myeloid leukaemia. Br J Haematol. 2004;127:76-84.

11. Leinoe EB, Hoffmann MH, Kjaersgaard E, Nielsen JD, Bergmann OJ, Klausen TW, et al. Prediction of haemorrhage in the early stage of acute myeloid leukemia by flow cytometric analysis of platelet function Br J Haematol. 2005;128(4):526-32.

12. Kuter DJ. Managing thrombocytopenia associated with cancer chemotherapy. Oncology (Williston Park). 2015;29(4):282-94.

13. Pogliani EM, Fantasia R, Lambertenghi-Deliliers G, Cofrancesco E. Daunorubicin and platelet function. Thromb Haemost. 1981;45(1):38-42.

14. Lanzi C, Banfi P, Ravagnani F, Gambetta RA. Diversity of effects of two antitumor anthracycline analogs on the pathway of activation of PKC in intact human platelets. Biochem Pharmacol. 1988;37(18):3497-504.

15. Foss $B$, Ulvestad $E$, Hervig $T$, Bruserud $\varnothing$. Effects of cytarabine and various anthracyclins on platelet activation: characterization of in vitro effects and their possible clinical relevance in acute myelogenous leukemia. Int J Cancer. 2002;97:106-14.

16. Moreau P, San Miguel J, Sonneveld P, Mateos MV, Zamagni E, Avet-Loiseau H, et al. Multiple myeloma: ESMO clinical practice guidelines for diagnosis, treatment and follow-up. Ann Oncol. 2017;28(suppl_4):iv52-61.

17. HOVON - the Haemato Oncology Foundation for Adults in the Netherlands. Trials (by type) 2017. Available from: http://www.hovon.nl/trials/trials-by-type/all.html.

18. HOVON - the Haemato Oncology Foundation for Adults in the Netherlands. Trials NHL 2017. Available from: http://www.hovon.nl/trials/trials-by-type/nhl.html.

19. American Cancer Society. How chemotherapy drugs work 2016 [updated February 11, 2016]. Available from: https://www.cancer.org/treatment/treatments-and-side-effects/treatment-types/ chemotherapy/how-chemotherapy-drugs-work.html.

20. CBO. Blood transfusion guidelines- the Netherlands. 2011.

21. Smeets EF, Heemskerk JW, Comfurius P, Bevers EM, Zwaal RF. Thapsigargin amplifies the platelet procoagulant response caused by thrombin. Thromb Haemost. 1993;70(6):1024-9.

22. Vogler M, Hamali HA, Sun XM, Bampton ETW, Dinsdale D, Snowden RT, et al. BCL2/BCL-XL inhibition induces apoptosis, disrupts cellular calcium homeostasis, and prevents platelets activation. Blood. 2011;117:7145-54. 
23. Solari FA, Mattheij NJ, Burkhart JM, Swieringa F, Collins PW, Cosemans JM, et al. Combined quantification of the global proteome, phosphoproteome, and proteolytic cleavage to characterize altered platelet functions in the human Scott syndrome. Mol Cell Proteomics. 2016;15(10):3154-69.

24. Kramer PA, Ravi S, Chacko B, Johnson MS, Darley-Usmar VM. A review of the mitochondrial and glycolytic metabolism in human platelets and leukocytes: Implications for their use as bioenergetic biomarkers. Redox Biol. 2014;2:206-10.

25. Mattheij NJ, Gilio K, van Kruchten R, Jobe SM, Wieschhaus AJ, Chishti AH, et al. Dual mechanism of integrin $\alpha$ llb $\beta 3$ closure in procoagulant platelets. J Biol Chem. 2013;288(19):13325-36.

26. van Kruchten R, Mattheij NJA, Saunders C, Feijge MA, Swieringa F, Wolfs JLN, et al. Both TMEM16Fdependent and TMEM16F-independent pathways contribute to phosphatidylserine exposure in platelet apoptosis and platelet activation. Blood. 2013;121(10):1850-7.

27. Lanza IR, Nair KS. Mitochondrial metabolic function assessed in vivo and in vitro. Curr Opin Clin Nutr Metab Care. 2010;13(5):511-7.

28. Larsen S, Nielsen J, Hansen CN, Nielsen LB, Wibrand F, Stride N, et al. Biomarkers of mitochondrial content in skeletal muscle of healthy young human subjects. J Physiol. 2012;590(14):3349-60.

29. Gilliam LAA, Fisher-Wellman KH, Lin CT, Maples JM, Cathey BL, Neufer PD. The anticancer agent doxorubicin disrupts mitochondrial energy metabolism and redox balance in skeletal muscle. Free Radic Biol Med. 2013;65:988-96.

30. Ma J, Kavelaars A, Dougherty PM, Heijnen CJ. Beyond symptomatic relief for chemotherapy-induced peripheral neuropathy: Targeting the source. Cancer. 2018;Epub ahead of print.

31. Zeuner A, Signore M, Martinetti D, Barturcci M, Peschle C, De Maria R. Chemotherapy-induced thrombocytopenia derives from the selective death of megakaryocyte progenitors and can be rescued by stem cell factor. Cancer Res. 2007;67(10):4767-73.

32. Riedl J, Kaider A, Marosi C, Prager GW, Eichelberger B, Assinger A, et al. Decreased platelet reactivity in patients with cancer is associated with high risk of venous thromboembolism and poor prognosis. Thromb Haemost. 2017;117(1):90-8.

33. Baaten CC, Ten Cate H, van der Meijden PE, Heemskerk JW. Platelet populations and priming in hematological diseases. Blood Rev. 2017;31(6):389-99.

34. Sorensen JC, Cheregi BD, Timpani CA, Nurgali K, Hayes A, Rybalka E. Mitochondria: Inadvertent targets in chemotherapy-induced skeletal muscle toxicity and wasting? Cancer Chemother Pharmacol. 2016;78(4):673-83.

35. Gouspillou G, Scheede-Bergdahl C, Spendiff S, Vuda M, Meehan B, Mlynarski H, et al. Anthracyclinecontaining chemotherapy causes long-term impairment of mitochondrial respiration and increased reactive oxygen species release in skeletal muscle. Sci Rep. 2015;5:8717.

36. Ichikawa $Y$, Ghanefar M, Bayeva M, Wu R, Khechaduri A, Naga Prasad SV, et al. Cardiotoxicity of doxorubicin is mediated through mitochondrial iron accumulation. J Clin Invest. 2014;124(2):617-30.

37. Al-Nasser IA. In vivo prevention of cyclophosphamide-induced Ca2+ dependent damage of rat heart and liver mitochondria by cyclosporin A. Comp Biochem Physiol A Mol Integr Physiol. 1998;121(3):209-14.

38. Prasad SB, Rosangkima G, Nicol BM. Cyclophosphamide and ascorbic acid-mediated ultrastructural and biochemical changes in Dalton's lymphoma cells in vivo. Eur J Pharmacol. 2010;645(1-3):47-54.

39. Kang PT, Chen CL, Ren P, Guarini G, Chen YR. BCNU-induced gR2 defect mediates S-glutathionylation of complex I and respiratory uncoupling in myocardium. Biochem Pharmacol. 2014;89(4):490-502.

40. Corona de la Peña N, Gutiérrez-Aguilar M, Hernández-Reséndiz I, Marín-Hernández Á, RodríguezEnríguez S. Glycoprotein Ib activation by thrombin stimulates the energy metabolism in human platelets. PLoS One. 2017;12(8):e0182374.

41. Bergmeier W, Piffath CL, Cheng G, Dole VS, Zhang Y, von Andrian UH, et al. Tumor necrosis factoralpha-converting enzyme (ADAM17) mediates GPIbalpha shedding from platelets in vitro and in vivo. Circ Res. 2004;95(7):677-83.

42. Zhang $Q$, Dong $T$, Li $P$, Wu MX. Noninvasive low-level laser therapy for thrombocytopenia. Sci Trans/ Med. 2016;8(349):349ra101.

43. Huijskens MJ, Wodzig WK, Walczak M, Germeraad WT, Bos GM. Ascorbic acid serum levels are reduced in patients with hematological malignancies. Results Immunol. 2016;6:8-10. 
44. Mackman N, Tilley RE, Key NS. Role of the extrinsic pathway of blood coagulation in hemostasis and thrombosis. Arterioscler Thromb Vasc Biol. 2007;27(8):1687-93.

45. Hoffbrand AV, Moss PAH. Essential Haematology. 6th ed: Wiley-Blackwell; 2011.

46. Giordano P, Molinari AC, Del Vecchio GC, Saracco P, Russo G, Altomare M, et al. Prospective study of hemostatic alterations in children with acute lymphoblastic leukemia. Am J Hematol. 2010;85(5): 325-30.

47. Briggs C, Kunka S, Hart D, Oguni S, Machin SJ. Assessment of an immature platelet fraction (IPF) in peripheral thrombocytopenia. Br J Haematol. 2004;126(1):93-9. 


\section{Appendix 6.A Supplemental methods}

\section{Materials}

Apyrase, bovine serum albumin (BSA), calcium chloride, the citrate synthase activity kit, dimethylsulfoxide, glucose, magnesium chloride and thrombin were obtained from Sigma (St. Louis, MO, USA). Collagen related peptide (CRP) was purchased from Dr. R. Farndale (Cambridge, UK). ABT-737, 2MeS-ADP and thapsigargin were from SantaCruz Biotechnology (Santa Cruz, CA, USA); fluorescein isothiocyanate (FITC)conjugated PAC-1 antibody against active integrin $\alpha_{11 b} \beta_{3}$ and FITC-conjugated antiCD61 antibody from Becton-Dickinson Bioscience (Franklin Lakes, NJ, USA). Alexa Fluor 546 conjugated human fibrinogen; Fluo-4 AM; $\mathrm{H}_{2}$ DCFDA and NuPAGE LDS sample buffer NOVEX were from Invitrogen Life Technologies (Bleiswijk, The Netherlands). FITC-conjugated anti-P-selectin antibody came from Beckman Coulter, rabbit anti- $\alpha$-tubulin antibody from Abcam (Cambridge, UK) and annexin-A5 FITCconjugated was from PharmaTarget (Maastricht, The Netherlands). FITC-conjugated anti-GPIb $\alpha$ antibody was from Sanquin (Amsterdam, the Netherlands) and the PEconjugated anti-GPVI antibody was from Biocytex (Marseille, France). Collagen type I came from Nycomed Pharma (Munich, Germany). Tetramethyl rhodamine methyl ester (TMRE) was from Anaspec (San Jose, CA, USA); Q-VD-Oph from Calbiochem (San Diego, CA, USA) and the fluorometric caspase-3 activity assay from R\&D Systems (Minneapolis, MN, USA). Prestained SDS-PAGE standards were from BioRad (Hercules, CA, USA) and SuperSignal West pico chemiluminescent substrate came from Thermo Scientific (Waltham, MA, USA). Actin FSL Activated PTT reagent, Thrombin reagent and Innovance D-dimer test were from Siemens Healthcare Diagnostics (Den Haag, The Netherlands). Antibody against kindlin-3 was purchased at Cell Signaling Technology (Danvers, MA, USA).

\section{Preparation of washed platelets and plasma}

Platelet isolation procedures were adapted for measurements with low platelet numbers (median $0.36 \times 10^{8}$ platelets per blood sample). Platelet-rich plasma (PRP) was obtained from citrated blood by centrifugation at $290 \mathrm{~g}$ for $2 \mathrm{~min}$. For preparation of washed platelets, PRP in eppendorf tubes was supplemented with 1:10 v/v acidic citrate dextrose $(A C D, 80 \mathrm{mM}$ trisodium citrate, $52 \mathrm{mM}$ citric acid and $180 \mathrm{mM}$ glucose) and centrifuged at $2230 \mathrm{~g}$ for $2 \mathrm{~min}^{1}$. Pelleted platelets were resuspended in Hepes buffer pH 6.6 (10 mM Hepes, $136 \mathrm{mM} \mathrm{NaCl}, 2.7 \mathrm{mM} \mathrm{KCl}, 2 \mathrm{mM} \mathrm{MgCl}, 0.1 \%$ glucose and $0.1 \%$ bovine serum albumin (BSA). After another centrifugation step (2230 $\mathrm{g}$ for $2 \mathrm{~min}$ ) in the presence of $1: 15 \mathrm{ACD}+1 \mathrm{U} / \mathrm{mL}$ apyrase, pelleted platelets were resuspended in Hepes buffer pH 7.45 (10 mM Hepes, $136 \mathrm{mM} \mathrm{NaCl}, 2.7 \mathrm{mM} \mathrm{KCl}$, 
$2 \mathrm{mM} \mathrm{MgCl}, 0.1 \%$ glucose and $0.1 \% \mathrm{BSA}$ ). Platelet count was determined with a Sysmex XP300/XN9000 thrombocounter (Kobe, Japan). Platelet-free plasma was prepared, as described before. ${ }^{1}$

\section{Whole blood cell count and coagulation tests}

Whole blood cell count in $\mathrm{K}_{2}$-EDTA anticoagulated blood was determined using a Sysmex XN-9000 analyzer. Platelet-free plasma was analyzed using a Sysmex CS-2100i analyzer and Dade reagents for: activated partial thromboplastin time (aPTT, Actin FSL), prothrombin time (PT, Innovin), thrombin time (Thrombin reagent), fibrinogen, D-dimers (Innovance) and von Willebrand factor antigen. Coagulation factor activities were determined, as described previously ${ }^{2,3}$. Normal pooled plasma from healthy controls was used as a reference.

\section{Flow cytometric analysis of platelet activation}

To measure platelet activation processes, washed platelets from patients and control subjects were normalized to $10 \times 10^{6}$ platelets $/ \mathrm{mL}$. We preferred to use platelets from healthy volunteers as controls and adapt the platelet count, since in cases of immune or congenital thrombocytopenia the underlying acquired or genetic alterations in platelets may also affect platelet functions. Platelets were activated with thrombin $(4 \mathrm{nM}), \mathrm{CRP}-\mathrm{XL}(10 \mu \mathrm{g} / \mathrm{mL})$ or $2 \mathrm{MeS}-\mathrm{ADP}(1 \mu \mathrm{M})$ in the presence of $2 \mathrm{mM} \mathrm{CaCl}_{2}$ for 15 min. Integrin $\alpha_{11 b} \beta_{3}$ activation and P-selectin expression were assessed, following labeling with FITC-conjugated PAC-1 antibody $(1.25 \mu \mathrm{g} / \mathrm{mL})$ or FITC-conjugated anti-Pselectin antibody $(5 \mu \mathrm{g} / \mathrm{mL})$, as described before ${ }^{4}$. Receptor expression was determined by post-labeling of platelets with anti-CD61 FITC conjugated antibody (1:10), anti-GPIba FITC conjugated antibody (1:20) or anti-GPVI PE conjugated antibody (1:20). For detection of apoptotic phosphatidylserine (PS) exposure, washed platelets $\left(10 \times 10^{6} / \mathrm{mL}\right)$ were stimulated with ABT-737 (5-10 $\left.\mu \mathrm{M}\right)$ for 150 min. ${ }^{5}$ Subsamples were stained with FITC-annexin A5 $(0.25 \mu \mathrm{g} / \mathrm{mL})$ in the presence of $2 \mathrm{mM}$ $\mathrm{CaCl}_{2}$. Platelets were pretreated with inhibitor or vehicle for $15 \mathrm{~min}$, where indicated. Samples were analyzed using a BD Accuri C6 flow cytometer and software (BectonDickinson Bioscience, Franklin Lakes, NJ, USA). ${ }^{5}$

To assess intracellular $\mathrm{Ca}^{2+}$ rises, washed platelets were loaded with the $\mathrm{Ca}^{2+}$ probe Fluo-4 AM $(8 \mu \mathrm{M})$ in the presence of Pluronic F-127 $(0.4 \mu \mathrm{g} / \mathrm{mL})$ for $45 \mathrm{~min}$, as described. ${ }^{5}$ After removal of excess probe by a wash step, Fluo-4-loaded platelets were resuspended into Hepes buffer $\mathrm{pH} 7.45\left(2 \times 10^{6} / \mathrm{mL}\right)$ supplemented with $2 \mathrm{mM}$ $\mathrm{CaCl}_{2}$, and then activated with thrombin $(4 \mathrm{nM}), \mathrm{CRP}-\mathrm{XL}(10 \mu \mathrm{g} / \mathrm{mL})$ or the SERCA pump inhibitor thapsigargin $(0.5 \mu \mathrm{M})$. Fluorescence changes were monitored in time, 
and expressed as increases over basal fluorescence, indicative of rises in cytosolic $\mathrm{Ca}^{2+}$ concentrations ${ }^{5}$.

\section{Platelet spreading on fibrinogen}

Washed glass coverslips were coated with fibrinogen $(100 \mu \mathrm{g} / \mathrm{mL})$ and blocked with $1 \%$ BSA. Washed platelets $\left(10 \times 10^{6} / \mathrm{mL}\right)$ were allowed to adhere and spread for 10 min, ${ }^{6}$ after which brightfield images were captured using a Zeiss LSM7 confocal linescanning microscope (Oberkochen, Germany) equipped with a 63x oil immersion objective. ${ }^{7}$ Spreading state per platelet was classified in three stages based on morphology: (i) filopodia, (ii) lamellipodia, or (iii) fully spread. The analysis was executed blinded.

\section{Mitochondrial function and morphology}

To detect mitochondrial depolarization, washed platelets from patients and controls $\left(10 \times 10^{6} / \mathrm{mL}\right)$ were loaded with the mitochondrial membrane potential dye, tetramethylrhodamine, ethyl ester (TMRE, $50 \mathrm{nM}$ ) for $30 \mathrm{~min}$ at room temperature. Using flow cytometry, mean fluorescence intensities of TMRE were assessed to evaluate the mitochondrial membrane potential. ${ }^{5}$

Mitochondrial respiration was determined by high-resolution respirometry with an Oroboros Oxygraph-2K (Oroboros Instruments, Innsbruck, Austria), at $37^{\circ} \mathrm{C}$ and roomair saturated oxygen tension, according to previously established methodology. ${ }^{8}$ Briefly, washed platelets were resuspended in buffer Z (105 mM K-MES, $30 \mathrm{mM} \mathrm{KCl}$, $10 \mathrm{mM} \mathrm{KH}{ }_{2} \mathrm{PO}_{4}, 1 \mathrm{mM}$ EGTA, $5 \mathrm{mM} \mathrm{MgCl}, 5 \mathrm{mg} / \mathrm{mL}$ BSA, $5 \mu \mathrm{M}$ pyruvate and $2 \mu \mathrm{M}$ malate, $\mathrm{pH}$ 7.1) and allowed to stabilize on ice for $30 \mathrm{~min}$. Washed platelets (final concentration: $50 \times 10^{6} / \mathrm{mL}$ ) were added to the respiration chamber, which contained buffer Z, supplemented with $5 \mathrm{mM}$ pyruvate, $0.5 \mathrm{mM}$ malate and $50 \mu \mathrm{g} / \mathrm{mL}$ saponin. After approximately $15 \mathrm{~min}$, when stable respiration was reached, the OXPHOS substrates ADP $(5 \mathrm{mM})$, glutamate $(10 \mathrm{mM})$, succinate $(10 \mathrm{mM})$ and cytochrome $\mathrm{c}$ $(5 \mathrm{mM})$ were sequentially added in 10 min increments. Oxygen concentration and oxygen flux (the negative time derivative of oxygen consumption) were monitored in real time.

As an independent read-out of mitochondrial content, citrate synthase activity was determined in lysates of washed platelets $\left(5 \times 10^{6}\right)$, using a colorimetric assay (Sigma, St. Louis, MO, USA). Enzymatic activity was determined at $37^{\circ} \mathrm{C}$ from absorbance changes at $412 \mathrm{~nm}$, according to the manufacturer's protocol.

For transmission electron microscopy, washed platelets in the presence of $10 \mathrm{nM}$ iloprost were fixed during $1 \mathrm{~h}$ with $1.5 \%$ glutaraldehyde in phosphate-buffered saline. Samples were then embedded in Epon and processed, as described before ${ }^{9}$. 


\section{Reactive oxygen species (ROS)}

To detect ROS, platelets $\left(10 \times 10^{6}\right.$ platelets $\left./ \mathrm{mL}\right)$ from patients and controls were loaded with $\mathrm{H}_{2}$ DCFDA $(10 \mu \mathrm{M})$ for 30 minutes at $37^{\circ} \mathrm{C}$. Subsequently, subsamples were taken and fluorescence was measured using flow cytometry. Platelets of healthy controls were stimulated with $100 \mu \mathrm{M} \mathrm{CCCP}$ as a positive control.

\section{Western blot analysis}

Washed platelets in Hepes buffer 7.45 were lysed (1:4) into 4x ice-cold lysis buffer (600 mM NaCl, 40 mM Tris, 4 mM EGTA, 4 mM EDTA, 4\% Nonidet-P40, pH 7.5). Protein content was quantified using a micro BCA protein assay (Thermo Scientific, Waltham, MA, USA). Samples were denatured by adding 1:4 NuPAGE LDS sample buffer, and subsequently heated at $95{ }^{\circ} \mathrm{C}$ for $5 \mathrm{~min}$. The denatured samples were separated on an $8 \%$ SDS-polyacrylamide gel, and then transferred to a PVDF blotting membrane. Immunoblotting was performed with antibodies against kindlin-3 (1:1000) or $\alpha$-tubulin (1:1000; loading control) overnight at $4^{\circ} \mathrm{C}$, followed by staining using a horseradish peroxidase (HRP) coupled secondary antibody for $1 \mathrm{~h}$ at room temperature. After incubation with SuperSignal West pico-chemiluminescent substrate, protein bands were visualized using an ImageQuant LAS-4000 mini system (Wauwatosa, WI, USA).

\section{Caspase activity}

Caspase activity was assessed in washed platelets $\left(10 \times 10^{6} / \mathrm{mL}\right)$ treated with vehicle or ABT-737 (10 $\mu \mathrm{M})$ for $90 \mathrm{~min}$ at $37^{\circ} \mathrm{C}$, as described before ${ }^{5}$. 


\section{Appendix 6.B Supplemental results}

Table S6.1 Coagulation parameters and factor activities of patient blood samples.

\begin{tabular}{lcc}
\hline Coagulation parameters & Patient cohort & Reference range \\
\hline Fibrinogen (g/L) & $4.2(3.4-5.1)$ & $1.7-4.0$ \\
d-Dimer (ng/mL) & $2166(1395-4276)$ & $<500$ \\
aPTT (s) & $29.2(26.5-33.4)$ & $23.0-32.0$ \\
PT (s) & $11.0(10.6-11.6)$ & $9.9-11.5$ \\
Thrombin time (s) & $15.8(14.9-16.7)$ & $15.0-22.0$ \\
vWF (\%) & $251(200-306)$ & $40-190$ \\
Prothrombin (\%) & $92(85-100)$ & $70-130$ \\
Factor V (\%) & $126(101-150)$ & $70-130$ \\
Factor VII (\%) & $58(47-73)^{*}$ & $70-130$ \\
Factor VIII (\%) & $172(144-197)$ & $50-200$ \\
Factor IX (\%) & $127(114-139)$ & $60-140$ \\
Factor X (\%) & $77(66-84)$ & $60-140$ \\
Factor XI (\%) & $106(85-120)$ & $60-140$ \\
Factor XII (\%) & $93(69-104)$ & $60-140$ \\
Factor XIII (\%) & $105(90-129)$ & $70-140$ \\
\hline
\end{tabular}

Factor activities were assayed relatively to Standard Human Plasma (SHP) and are expressed as \% activity. Samples without prior platelet transfusion. (Medians + interquartile ranges)

Table S6.2 Chemotherapeutic drugs taken by included patients, classified according to the main action mechanism.

\begin{tabular}{ll}
\hline Class & Drug \\
\hline A & daunorubicin, doxorubicin, etoposide, mitoxantrone, idarubicin \\
B & cytarabine (ara-C), fludarabine \\
C & busulfan, carmustine (BCNU), cyclofosfamide, melphalan \\
D & vincristin \\
E & lenalidomide, tosedostat, retuximab \\
\hline
\end{tabular}

Drug classes: A, antitumor antibiotics \& topo-isomerase II inhibitors; B, antimetabolites; C, alkylating agents; $D$, mitotic inhibitors; $E$, other.

Table S6.3 Numbers of included patients taking chemotherapeutic drugs of different classes, alone or in combination.

\begin{tabular}{lccccc}
\hline Diagnosis & $\begin{array}{c}\text { A } \\
\text { anti-tumor \& } \\
\text { TI inhibitor }\end{array}$ & $\begin{array}{c}\text { B } \\
\text { anti-metabolite }\end{array}$ & $\begin{array}{c}\text { Drug class } \\
\text { alkylating agent }\end{array}$ & $\begin{array}{c}\text { D } \\
\text { mitotic } \\
\text { inhibitor }\end{array}$ & $\begin{array}{c}\text { E } \\
\text { other }\end{array}$ \\
\hline AML/ALL, $n$ & 24 & 29 & 11 & 1 & 10 \\
Lymphoma, $n$ & 13 & 15 & 15 & 1 & 1 \\
Multiple myeloma, $n$ & 0 & 0 & 21 & 0 & 0 \\
Other, $n$ & 0 & 1 & 3 & 0 & 0 \\
Total, $n(\%)$ & $37(25.5 \%)$ & $45(31.0 \%)$ & $50(34.5 \%)$ & $2(1.4 \%)$ & $11(7.6 \%)$ \\
\hline
\end{tabular}

For the list of individual drugs, see Table S6.1. 
Table S6.4 Hematologic parameters of blood samples from patients before and $1 \mathrm{~h}$ after platelet transfusion.

\begin{tabular}{lcc}
\hline Parameter & Before transfusion & After transfusion \\
\hline Leukocyte count $\left(\times 10^{9} / \mathrm{L}\right)$ & $0.14(0.04-0.41)$ & $0.12(0.04-0.44)$ \\
Hemoglobin $(\mathrm{mM})$ & $5.6(5.1-6.2)$ & $5.2(4.7-5.7)$ \\
Platelet count $\left(\times 10^{9} / \mathrm{L}\right)$ & $6(4-8)$ & $33(24.3-43.8)$ \\
Absolute immature platelet number $\left(\times 10^{9} / \mathrm{L}\right)$ & $0.21(0.07-0.38)$ & $1.17(0.69-1.60)$ \\
Immature platelet fraction $(\%)$ & $3.8(2.2-6.3)$ & $3.6(2.7-4.2)$ \\
Corrected count increment & $n . a$. & $14.8(11.3-18.0)$ \\
\hline
\end{tabular}

Data are expressed as medians (interquartile ranges)

Figure S6.1 Variable impairment in platelet responsiveness is independent of chemotherapy treatment regimen of patients.

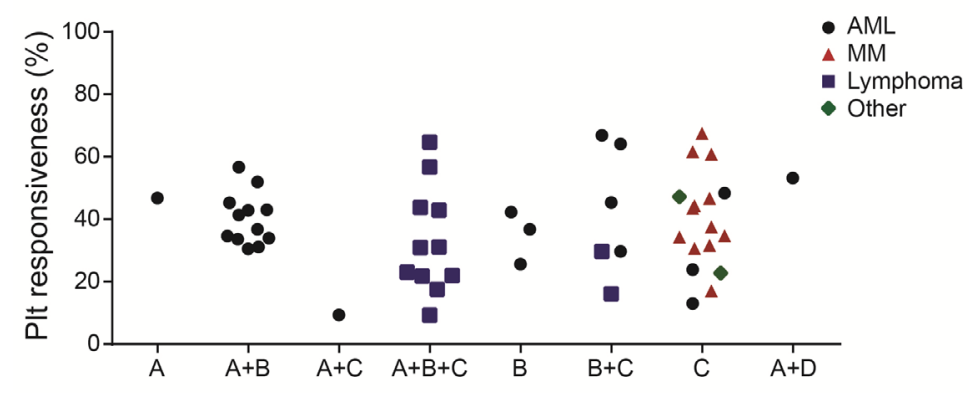

Integrin activation and P-selectin expression of washed platelets from patients was determined, as for Figure 1. Overall platelet responsiveness was defined per patient as the mean of percentages of platelets positive for integrin activation and P-selectin after stimulation with thrombin, CRP-XL or ADP. Patients were grouped based on treatment with chemotherapeutics into one of more classes (see Table S6.2): A, antitumor antibiotics \& topo-isomerase inhibitors; B, antimetabolites; C, alkylating agents; D, alkaloids. Data from 52 patients ( $A M L: n=25$, multiple myeloma: $n=12$, lymphoma: $n=13$, other: $n=2$ ). 
Figure S6.2 Transfusion partly normalizes platelet responsiveness in chemotherapy treated patients with thrombocytopenia.

\section{A}

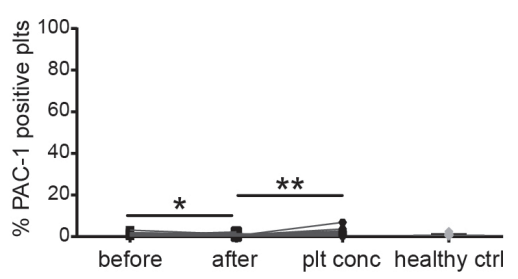

B

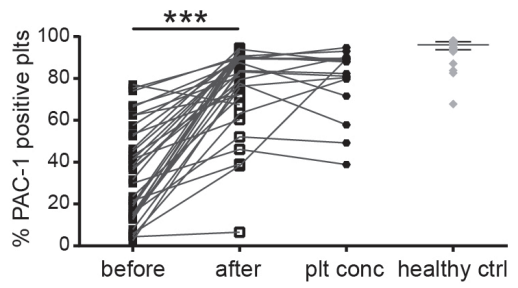

C

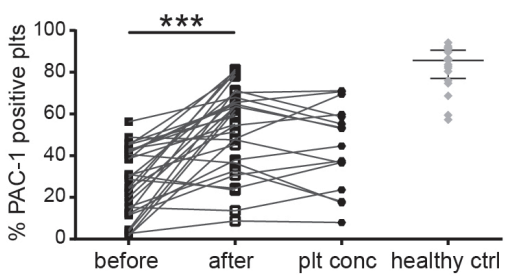

D

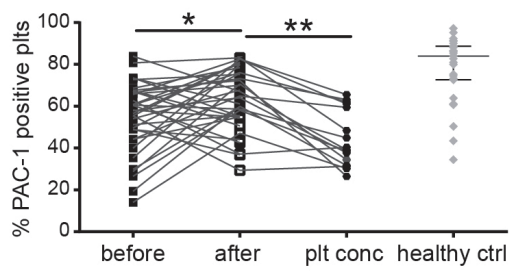

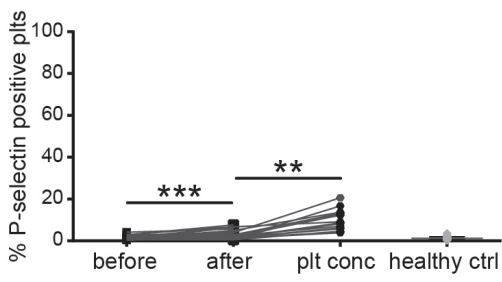

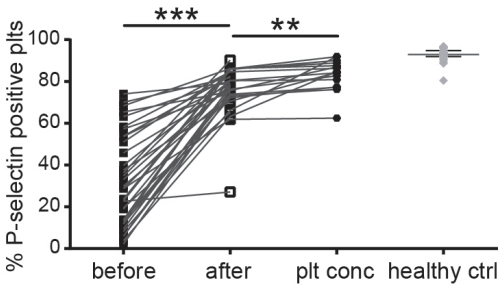

Washed platelets $\left(10 \times 10^{6} / \mathrm{mL}\right)$ analyzed from healthy controls, patients with chemotherapy-induced thrombocytopenia before and after transfusion, and from the transfused platelet concentrates. Integrin $\alpha_{\| 1 b} \beta_{3}$ activation and P-selectin expression were measured in resting platelets (A), and after stimulation with thrombin (B), CRP-XL (C) or 2MeS-ADP (D). Median values with IQR for patients ( $n=36$ : treatment classes: $A+B: n=7 ; A+B+C: n=10 ; B+C: n=4 ; C: n=11)$ and platelet concentrates $(n=16)$ and control subjects $(n=27)$; ${ }^{*} p<0.05,{ }^{* *} p<0.01$ and ${ }^{* * *} p<0.001$. 
Figure S6.3 Ex vivo treatment with chemotherapeutic agents does not influence platelet functionality.
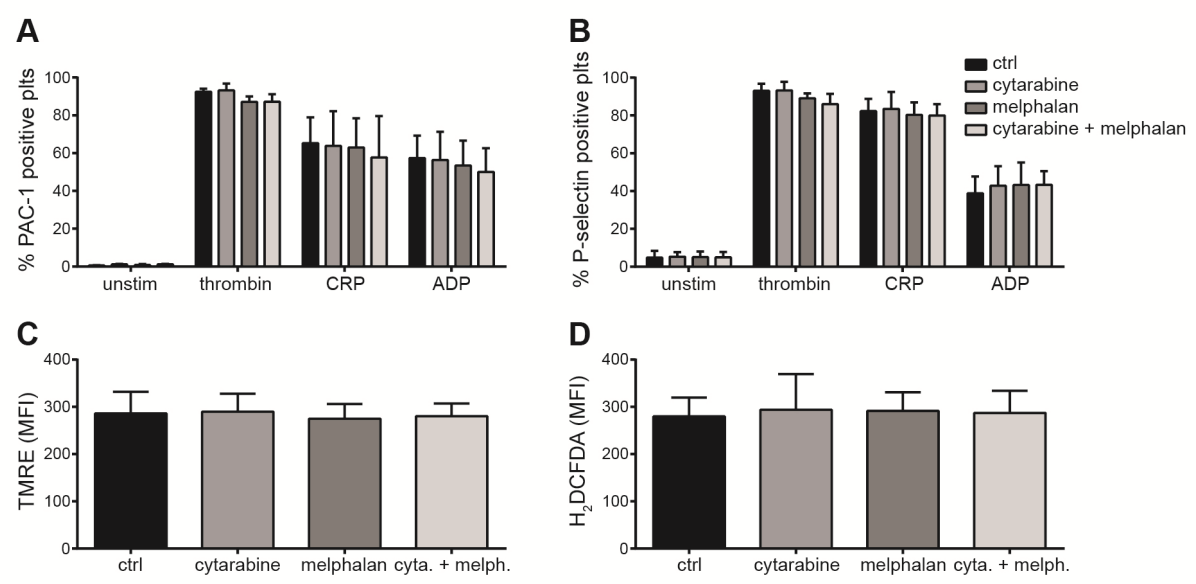

Whole blood from healthy control subjects was incubated with clinically relevant concentrations of cytarabine $(70 \mu \mathrm{g} / \mathrm{mL})$ and/or melphalan $(50 \mu \mathrm{g} / \mathrm{mL})$ for $60 \mathrm{~min}$ at $37^{\circ} \mathrm{C}$. Subsequently, platelets were isolated, and platelet reactivity (Integrin $\alpha_{11 b} \beta_{3}(A)$ and P-selectin expression (B)), mitochondrial membrane potential (TMRE) (C), and ROS ( $\mathrm{H}_{2}$ DCFDA) (D) were measured. Results are depicted as means plus $S D, n=4$, $* p<0.05$.

\section{References}

1. Cauwenberghs S, Feijge MA, Hageman G, et al. Plasma ecto-nucleotidases prevent desensitization of purinergic receptors in stored platelets. Transfusion 2006;46(6):1018-28.

2. van Beers JJ, van Egmond LT, Wetzels RJ, et al. Increased coagulation and fibrinolytic potential of solvent-detergent plasma: a comparative study between Omniplasma and fresh frozen plasma. Vox Sang 2016;111(1):33-42.

3. Schols SE, Lancé MD, Feijge MA, et al. Impaired thrombin generation and fibrin clot formation in patients with dilutional coagulopathy during major surgery. Thromb Haemost 2010;103(2):318-28.

4. Mattheij NJ, Gilio K, van Kruchten R, et al. Dual mechanism of integrin aiibb3 closure in procoagulant platelets. J Biol Chem 2013;288(19):13325-36.

5. van Kruchten R, Mattheij NJA, Saunders C, et al. Both TMEM16F-dependent and TMEM16Findependent pathways contribute to phosphatidylserine exposure in platelet apoptosis and platelet activation. Blood 2013;121(10):1850-7.

6. Nergiz-Unal R, Lamers MM, van Kruchten R, et al. Signaling role of CD36 in platelet activation and thrombus formation on immobilized thrombospondin or oxidized low-density lipoprotein. J Thromb Haemost 2011;9(9):1835-46.

7. Swieringa F, Baaten CC, Verdoold R, et al. Platelet control of fibrin distribution and microelasticity in thrombus formation under flow. Arterioscler Thromb Vasc Biol 2016;36(4):692-9.

8. Sjövall F, Morota S, Hansson MJ, et al. Temporal increase of platelet mitochondrial respiration is negatively associated with clinical outcome in patients with sepsis. Crit Care 2010;14(6):R214.

9. van Nispen tot Pannerden $\mathrm{H}$, de Haas F, Geerts $W$, et al. The platelet interior revisited: electron tomography reveals tubular alpha-granule subtypes. Blood 2010;116(7):1147-56. 



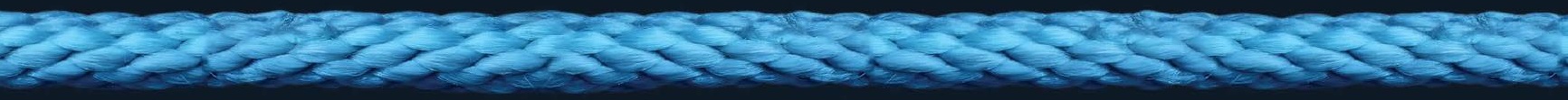




\section{CHAPTER 7}

Fibrinolysis in patients with chemotherapy induced thrombocytopenia and the effect of platelet transfusion

Floor C.J.I. Heubel-Moenen, Yvonne M.C Henskens, Paul W.M. Verhezen, Rick J. H. Wetzels, Harry C. Schouten, Erik A.M. Beckers 


\section{Abstract}

\section{Introduction}

Bleeding events in chemotherapy induced thrombocytopenic (CIT) patients with similar platelet counts might be influenced by changes in clot lysis potential.

\section{Aim}

In this observational study we investigated thromboelastographic lysis parameters, alterations in clot strength and susceptibility to clot lysis in CIT patients and identified factors associated with fibrinolytic profiles. Furthermore, the effects of platelet transfusions were evaluated.

\section{Methods}

Independent determinants of TPA-ROTEM lysis parameters were identified with multivariable linear regression. Clot formation, strength and lysis parameters were compared with results of healthy individuals. Characteristics of CIT patients with and without hyperfibrinolytic profiles were compared. tPA-ROTEM results before, 1 hour and 24 hours after platelet transfusion were compared.

\section{Results}

A total of 72 consecutive CIT patients were included. tPA-ROTEM lysis parameters correlated with changes in fibrinolytic proteins. Compared to healthy individuals, clot formation time was longer, maximum clot firmness was weaker and lysis times were shorter. CIT patients had low TAFI levels and forty percent exhibited hyperfibrinolytic profiles. Platelet transfusions resulted in less hyperfibrinolytic profiles in many, but not all CIT patients. Patients without hyperfibrinolytic profiles had higher fibrinogen, FVIII and $\alpha 2-A P$ levels.

\section{Conclusion}

tPA-ROTEM can be used as a fast and reliable assay to detect hyperfibrinolytic profiles in CIT patients. CIT patients have weaker clots which are more susceptible to clot lysis compared to healthy individuals. Besides platelets, other factors are likely to influence clot susceptibility to fibrinolysis in CIT patients. The impact of a hyperfibrinolytic TPAROTEM profile on bleeding remains to be investigated. 


\section{Introduction}

For adequate hemostasis the formation and sustainability of a stable clot containing platelets, fibrinogen, red blood cells and fibrin are essential ${ }^{1}$. Patients with chemotherapy induced thrombocytopenia (CIT) are at risk of bleeding and prophylactic platelet transfusions are widely used in CIT patients with a platelet count below $10 \times 10^{9} / \mathrm{L}$. Nonetheless, the effectiveness of platelet transfusions to prevent bleeding in CIT with platelets counts above 5,000 remains under debate ${ }^{2,3}$. On the one hand, despite prophylactic platelet transfusions, a significant number of patients will still bleed, while, on the other hand, a significant number of thrombocytopenic patients do not bleed when prophylactic platelet transfusions are withheld ${ }^{2,4}$. Hence, platelet count alone appears to be a poor predictor of bleeding. Other factors associated with severe bleeding in CIT patients are low hematocrit, low absolute immature platelet number (AIPN), prolonged activated partial thromboplastin time (APTT), prolonged prothrombin time (PT), recent allogeneic stem cell transplantation (SCT), infection, and previous bleeding, ${ }^{3,5-7}$. Clot formation and clot strength in thrombocytopenia have been investigated. Recent studies demonstrated that, in reconstituted thrombocytopenic blood, clot formation is impaired and cloth strength is reduced. This improves after in vitro addition of fibrinogen and factor $\mathrm{XIII-10}$. Two other studies showed that, in CIT patients, clot firmness is reduced, but improves after platelet transfusions ${ }^{6,11}$.

Not much is known about clot lysis and clot sustainability in CIT patients. Clot lysis, or fibrinolysis, is a highly regulated process involving the conversion of plasminogen by tissue plasminogen activator (tPA) into plasmin, which cleaves fibrin into soluble degradation products. Fibrinolysis is inhibited by $\alpha 2$-antiplasmin, plasminogen activator inhibitor-1 and 2 (PAI-1 and 2) and attenuated by thrombin-activatable fibrinolysis inhibitor (TAFI) (Figure 1.2). Since clot architecture is a key determinant of clot lysis ${ }^{1}$, the weaker clots in CIT patients might be more susceptible to fibrinolytic activity. Indeed, a higher susceptibility of clots, in reconstituted thrombocytopenic blood, for tPA-induced fibrinolysis has been shown in vitro ${ }^{8}$. Also, since platelets contain plasminogen activator inhibitor-1 (PAI-1) and alpha-2-antiplasmin ( $\alpha 2-A P)$ in their $\alpha$-granules, these concentrations might be reduced in CIT patients. The less inhibitory activity may enhance fibrinolytic activity. However, this might be compensated by higher fibrinogen levels which are often seen in patients with malignancies ${ }^{12,13}$. Differences in fibrinolytic potential might explain the clinical bleeding variability observed in CIT patients with similar platelet counts, but this has not been investigated yet. 
As clot lysis is influenced by several components, assessing in vivo clot lysis is challenging ${ }^{14-16}$. The clot lysis time assay (CLT) was developed as a plasmatic test and is able to detect hypofibrinolysis and increased risk of thrombosis ${ }^{17-19}$. Although it has been tested less extensively as a measure for hyperfibrinolysis and bleeding tendency, it appears to be non-predictive for bleeding in $\mathrm{VWD}^{20}$. Another and different approach to measure clot lysis is by the use of whole blood rotational thromboelastography with addition of recombinant tPA (tPA-ROTEM). This assay has been developed in our center to evaluate clot susceptibility to fibrinolysis following the addition of tPA ${ }^{15}$. tPA-ROTEM has been shown to provide a rapid evaluation of the kinetics of clot formation and fibrinolysis in whole blood ${ }^{15,21}$.

\section{Objectives}

In this observational, prospective study (Dutch Trial Register NL9645) we investigated whether or not the tPA-ROTEM lysis parameters lysis onset time (LOT), lysis time (LT) and delta lysis speed (LS) are in agreement with the changes in fibrinolytic proteins associated with fibrinolysis in CIT patients. Furthermore, we assessed clot strength and susceptibility to TPA induced clot lysis in CIT patients and compared the results to healthy individuals. We also tried to determine subgroups within the total CIT patient group with comparable platelet counts, based on clot lysis potential and looked for contributing factors explaining the different lysis profiles. Finally, the effects of platelet transfusions on clot strength and clot lysis were evaluated.

\section{Material and methods}

\section{Study population}

Patients admitted to the hematology ward of the Maastricht University Medical Centre, were included from November 2014 until January 2017. Eligible patients were $\geq 18$ years of age, received chemotherapy for treatment of a hematologic malignancy and consequently had or were expected to have thrombocytopenia (platelet count $\left.\leq 50 \times 10^{9} / \mathrm{L}\right)$. According to standard practice, patients received prophylactic platelet transfusions (leukocyte-depleted pooled buffy coat from 5 whole blood donors), when the morning platelet count was below $10 \times 10^{9} / \mathrm{L}$.

Patient exclusion criteria were: sepsis, splenomegaly, signs of active bleeding at the time of blood withdrawal, and/or use of antithrombotic medication. Results were compared with TPA-ROTEM results from 40 healthy individuals (as described in Kuiper et al. $^{15}$ ). The study was approved by the local ethics committee. All participating 
patients and healthy individuals gave written informed consent according to the Helsinki declaration.

\section{Blood collection}

Blood samples were obtained via a central venous line, after flushing with $100 \mathrm{ml} \mathrm{NaCl}$ $0.9 \%$. A total amount of $17.5 \mathrm{ml}$ was obtained at three moments during regular blood withdrawal; before platelet transfusion, 1 hour and 24 hours after transfusion. Blood was collected in one $7.2 \mathrm{mg} \mathrm{K} \mathrm{K}_{2}$ EDTA tube of $4.0 \mathrm{ml}$ (BD Vacutainer, Becton Dickinson, Plymouth, UK) and three $3.2 \%$ citrate tubes of $4.5 \mathrm{ml}$ (BD Vacutainer, Becton Dickinson, Plymouth, UK). Platelet poor plasma (PPP) was obtained by centrifugation of citrated blood at 2,500 x g for $5 \mathrm{~min}$ and then at $10,000 \mathrm{xg}$ for $10 \mathrm{~min}$ at $18^{\circ} \mathrm{C}$.

Complete blood count, fibrinogen measurements and the TPA-ROTEM were performed within 2 hours after blood collection. All other tests were performed batch-wise in stored PPP $\left(-80^{\circ} \mathrm{C}\right)$, which was frozen in aliquots within 2 hours after blood collection.

\section{Laboratory measurements}

Hemoglobin $(\mathrm{Hb})$, platelet count and white blood cell (WBC) measurements were performed on a Sysmex XN 9000 analyzer (Sysmex Corporation, Kobe, Japan). PT (Innovin, Siemens), APTT (ActinFSL, Siemens), thrombin time (Thromboclotin Siemens), fibrinogen (Clauss method, Thrombin Reagent, Siemens) and FXIII activity (Berichrom FXIII, Siemens) measurements were performed on a Sysmex CS 2100i (Sysmex Corporation, Kobe, Japan). Levels of FVIII, FII (neoplastine R), FV, FVII, FVIII, FIX, FX (neoplastine R) and FXI were measured with a clotting assay using factordepleted plasma on a Sta-R apparatus (Stago Diagnostics, Paris, France). Levels of VWF antigen and activity were determined on a Sysmex CS2100i analyzer (VWF Reag and VWF Ac Reagens, Siemens) Plasminogen (Stachrom Plasminogen) and $\alpha 2-A P$ (Stachrom Antiplasmin) were performed by chromogenic assays on Sta- $R$, (Stago, Paris). Bio-immunoassays were used to measure tissue plasminogen activator (tPA) activity, plasminogen activator inhibitor-1 (PAI-1) activity and thrombin activatable fibrinolysis inhibitor (TAFI) zymogen (Zymutest, Hyphen, Biomed).

The tPA-ROTEM was performed according to earlier published methods ${ }^{15}$ on the ROTEM $^{\circledR}$ device (ROTEM $^{\circledR}$, TEM International $\mathrm{GmbH}$, Munich, Germany), within 2 hours of blood withdrawal. Tissue Factor, $\mathrm{CaCl}_{2}$ and rtPA (Actilyse) were added to the whole blood (to obtain final concentrations of $35 \mathrm{pM} \mathrm{TF}, 10 \mathrm{mM} \mathrm{CaCl}_{2}$ and $125 \mathrm{ng} / \mathrm{mL} \mathrm{rtPA}$ ). The device temperature was set to $37^{\circ} \mathrm{C}$ and maximum runtime was 120 minutes. The following tPA-ROTEM parameters were analysed: CT (clotting time in seconds), CFT (clot formation time in seconds), MCF (maximum clot firmness in 
millimetres), $\alpha$ ( $\alpha$-angle in degrees), LOT (lysis onset time in seconds; time taken for amplitude to decrease by $15 \%$ of MCF), LT (lysis time in seconds; time taken for amplitude to drop to $10 \%$ of MCF). Furthermore, to investigate the speed of clot lysis, delta lysis speed (LS) was calculated at two different moments using the tPA-ROTEM data: delta LS 100-85, defined as the decline in \%/min between MCF and LOT, and the delta LS 85-10, defined as the decline in \%/min between LOT and LT ${ }^{15}$. See Figure 7.1.

\section{Figure 7.1 Schematic representation of tPA-ROTEM assay.}

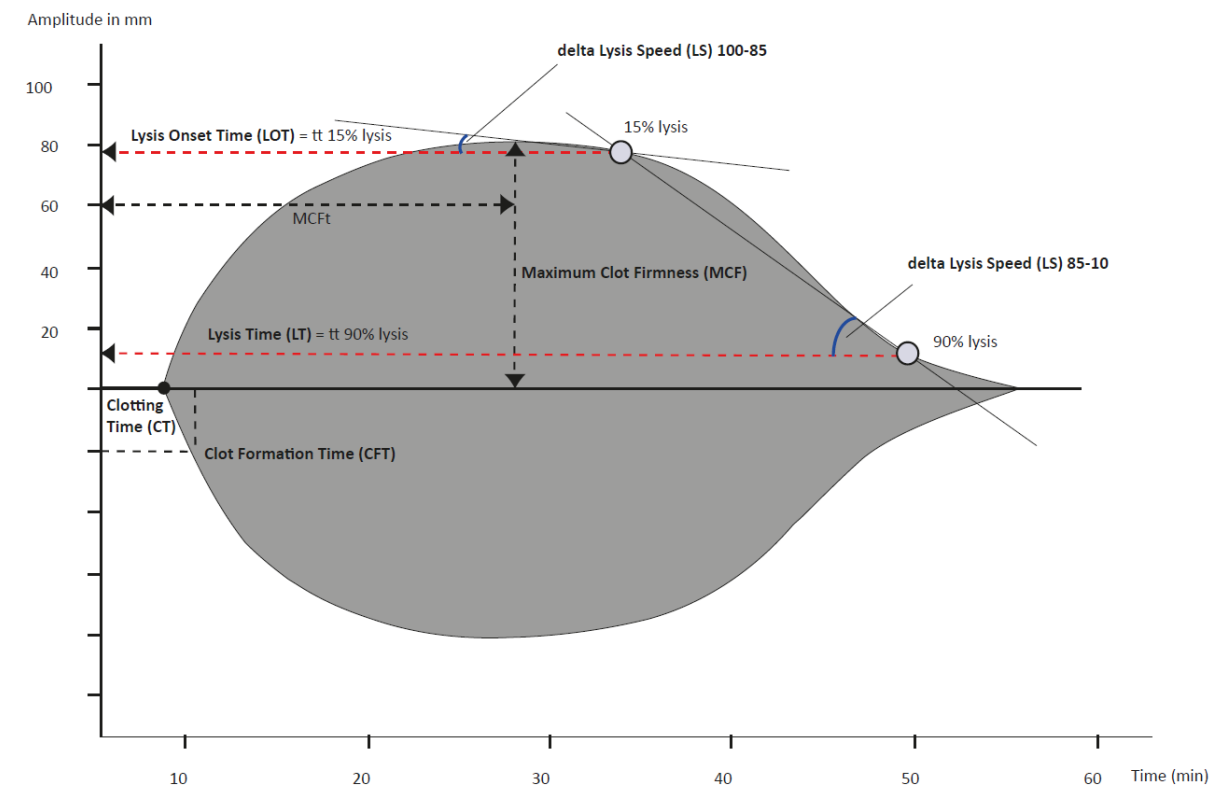

Important fibrinolysis parameters are illustrated. Delta lysis speed $100-85$ is defined as the decline in \%/min between MCF and LOT, delta lysis speed $85-10$ is defined as the decline in \%/min between LOT and LT. CT, clotting time; CFT, clot formation time; MCF, maximum clot firmness; LOT, lysis onset time; LS, lysis speed; LT, lysis time.

\section{Definition of hyperfibrinolysis as detected by tPA-ROTEM}

There is no 'gold standard' method for the detection of hyperfibrinolysis. ROTEM (EXTEM) has been used to determine the hyperfibrinolytic profile in trauma patients ${ }^{22}$. Previous studies investigating tPA-ROTEM lysis parameters showed that a decreased LOT has a good correlation with increasing tPA levels in vitro reflecting a hyperfibrinolytic profile. Even more, a decreased LOT was found to be a fast marker for fulminant hyperfibrinolysis in a patient population with out-of hospital cardiac arrest $^{21,23}$. Kuiper et al. clinically validated the tPA-ROTEM lysis parameters in different 
patient groups. Hypofibrinolytic profiles in patients with early sepsis and patients undergoing cardiothoracic surgery after tranexamic acid were reflected in a prolonged LOT, LT and a reduced delta $\mathrm{LS}^{15}$.

The cut-off values for a hyperfibrinolytic profile as measured by the tPA-ROTEM were defined by a LOT of less than 1560 seconds, a LT of less than 2100 seconds, a delta LS $100-85$ of more than $1.5 \% / \mathrm{min}$ and a delta LS $85-10$ of more than $9.8 \% / \mathrm{min}$. The cut-off values for a hypofibrinolytic profile were defined as a LOT of more than 2940 seconds, a LT of more than 4620 seconds, a delta LS 100-85 of less than $0.5 \% / \mathrm{min}$ and a delta LS $85-10$ of less than $2.5 \% / \mathrm{min}^{15}$. By applying these values, the lysis parameters were correlated with fibrinolytic proteins in this study. Figure 7.2 summarizes the changes in TPA-ROTEM lysis parameters and the corresponding changes in fibrinolytic proteins associated with a hypo- or hyperfibrinolytic profile.

Figure 7.2 TPA-ROTEM lysis parameters and fibrinolytic proteins.

\begin{tabular}{|c|c|c|c|c|c|c|c|c|c|c|c|}
\hline & LOT & LT & delta LS & a2-AP & TAFI & PAI-1 & Fibrinogen & tPA & Plasminogen & FII & FXIII \\
\hline $\begin{array}{c}\text { Hyperfibrinolytic } \\
\text { profile }\end{array}$ & $\downarrow$ & $\downarrow$ & $\uparrow$ & $\downarrow$ & $\downarrow$ & $\downarrow$ & $\downarrow$ & $\uparrow$ & $\uparrow$ & $\downarrow$ & $\downarrow$ \\
\hline $\begin{array}{c}\text { Hypofibrinolytic } \\
\text { profile }\end{array}$ & $\uparrow$ & $\uparrow$ & $\downarrow$ & $\uparrow$ & $\uparrow$ & $\uparrow$ & $\uparrow$ & $\downarrow$ & $\downarrow$ & $\uparrow$ & $\uparrow$ \\
\hline
\end{tabular}

Expected changes in TPA-ROTEM lysis parameters and fibrinolytic proteins associated with a hypo- or hyperfibrinolytic profile. $\alpha 2-\mathrm{AP}$, alfa-2-antiplasmin; tPA, tissue plasminogen activator; PAl-1, plasminogen activator inhibitor-1; TAFI, thrombin activatable fibrinolysis inhibitor; F, factor; LOT, lysis onset time; LT, lysis time; LS, lysis speed.

\section{Statistical analysis}

Continuous variables were presented as either mean \pm standard deviation for normally distributed traits or median \pm interquartile range otherwise. Categorical variables were expressed as counts and percentages. Differences between samples of healthy individuals and patients, and difference between samples of patients with and patients without a hyperfibrinolytic profile, were tested with an unpaired t-test in case of normal distribution, with the Mann-Whitney $U$ test in case of not normal distribution and with a Fisher's exact test in case of categorical variables.

Differences between samples before and after platelet transfusions were tested with the paired T-test in case of normal distribution and the Wilcoxon matched pair signed rank test in case of not normal distribution. Differences in proportions were tested with the Chi square test. Correlations were visualized with scatterplots and tested with Spearman Rank Order Correlation. Statistical significance was assumed at $p$ value $<0.05$. Differences between samples before, 1 hour and 24 hours after platelet 
transfusions were tested with the on way repeated ANOVA in case of normal distribution, the Friedman/Wilcoxon matched pair signed rank test in case of not normal distribution and McNemar in case of categorical variables. Bonferroni adjustment was made to the alpha value to control for type 1 errors. The adjusted alpha value was $0.017(0.05 / 3)$.

Multivariable linear regression was used to identify independent determinants of the TPA-ROTEM lysis parameters LOT, LT and delta LS, corrected for age and gender. Included predictor variables were fibrinogen, FII, FVII, FXIII, plasminogen, $\alpha 2-A P$, TPA, PAI-1, TAFI, hemoglobin level, platelet count and leukocyte count. Since the variables included in the model have different measurement units or measurement ranges, we used standardised regression coefficients (Beta) to compare relative strengths of the various determinants within the model. The standardised regression coefficient of a determinant indicates the increase in standard deviations of the outcome variable when the determinant increases with one standard deviation and all other variables in the model remain unchanged. Only patients for whom values of all predictor variables and test outcomes were available were included in the regression models. Data were missing in case of a partially failed blood withdrawal, or when no blood was left to repeat the laboratory assay when it initially failed. Statistical analyses were performed with IBM SPSS statistics version 24.0.

\section{Results}

\section{Baseline characteristics and determinants of tPA ROTEM lysis parameters in CIT patients}

A total of 72 consecutive CIT patients were included. Timing of the evaluations was before transfusion $(n=72), 1$ hour after transfusion $(n=57)$ and 24 hours after transfusion ( $n=45)$. TPA-ROTEM measurements of 40 healthy individuals were available ${ }^{15}$.

Characteristics and baseline laboratory values of $72 \mathrm{CIT}$ patients before transfusion are shown in Table 7.1. Mean age was $58 \pm 12$ years and 42/72 (58\%) patients were male. Most patients were diagnosed with acute leukemia (43\%) or multiple myeloma (32\%) and were treated with high dose chemotherapy followed by autologous stem cell transplantation (58\%) or chemotherapy alone (31\%). Hemoglobin level, leukocyte and platelet count were low due to treatment with chemotherapy. Conventional coagulation tests (APTT and PT) were normal. Fibrinogen level was above the normal range but showed a large variation. D-dimer level was 4-5 fold above the normal range. FVIII levels were high, as were VWF activity and antigen levels. Evaluation of 
fibrinolytic proteins showed low TAFI levels, all other fibrinolytic proteins were within the normal range.

Table 7.1 Patients characteristics and baseline laboratory values $(n=72)$.

\begin{tabular}{|c|c|c|}
\hline Patient characteristics & & mean \pm SD, $n(\%)$ \\
\hline Age in years & & $58 \pm 12$ \\
\hline Male & & $42(58)$ \\
\hline \multicolumn{3}{|l|}{ Diagnosis } \\
\hline Acute leukemia (AML/ALL) & & $31(43)$ \\
\hline Multiple myeloma & & $23(32)$ \\
\hline Non Hodgkin lymphoma & & $12(17)$ \\
\hline Other & & $6(8)$ \\
\hline \multicolumn{3}{|l|}{ Treatment } \\
\hline Chemotherapy & & $22(31)$ \\
\hline Autologous stem cell transplantation & & $42(58)$ \\
\hline Allogeneic stem cell transplantation & & $8(11)$ \\
\hline Height $(m)$ & & $1.73 \pm 0.09$ \\
\hline Weight $(\mathrm{kg})$ & & $75 \pm 12$ \\
\hline Baseline laboratory values & normal range & mean \pm SD, median [IQR] \\
\hline Hemoglobin (mmol/L) - male & $8.2-11.0$ & $5.8 \pm 0.7$ \\
\hline Hemoglobin (mmol/L) - female & 7.3-9.7 & $5.8 \pm 0.6$ \\
\hline Leukocytes $\left(\times 10^{9} / \mathrm{L}\right)$ & $3.5-11$ & $0.14[0.03-0.49]$ \\
\hline Thrombocytes (x10 $/ \mathrm{L})$ & $130-350$ & $6[4-9]$ \\
\hline $\operatorname{APTT}(s)$ & 23-32 & $31 \pm 5$ \\
\hline $\mathrm{PT}(\mathrm{s})$ & $9.9-11.5$ & $11.5 \pm 1.4$ \\
\hline Thrombin time (s) & $15-22$ & $16 \pm 1$ \\
\hline D-dimer (ng/ml) & $<500$ & 2387 [1641-4391] \\
\hline PAI-1 activity (ng/ml) & $<10.7$ & $0.60[0.29-1.02]$ \\
\hline TAFI (\%) & $100-172$ & $87 \pm 22$ \\
\hline$\alpha 2-\mathrm{AP}(\%)$ & $80-130$ & $101 \pm 16$ \\
\hline Plasminogen (\%) & $75-160$ & $90 \pm 17$ \\
\hline tPA activity (ng/ml) & $1-20$ & $6[5-10]$ \\
\hline FXIII & $60-140$ & $102 \pm 26$ \\
\hline FII (\%) & $60-140$ & $91 \pm 14$ \\
\hline Fibrinogen (g/dl) & $1.7-4.0$ & $4.6 \pm 1.5$ \\
\hline FV (\%) & $60-140$ & $130 \pm 31$ \\
\hline FVII (\%) & $60-140$ & $60 \pm 18$ \\
\hline FVIII (\%) & $50-150$ & $172 \pm 45$ \\
\hline FIX (\%) & $60-140$ & $127 \pm 27$ \\
\hline FX (\%) & $60-140$ & $74 \pm 15$ \\
\hline FXI (\%) & $60-140$ & $106 \pm 24$ \\
\hline VWF act (\%) & $50-150$ & $256 \pm 85$ \\
\hline VWF ant (\%) & $50-150$ & $256 \pm 97$ \\
\hline
\end{tabular}

$\alpha 2-A P$, alfa-2-antiplasmin; act, activity; ALL, acute lymphatic leukemia; AML, acute myeloid leukemia; ant, antigen; APTT, activated partial thrombin time; m, meters; kg, kilogram; L, liters; PT, prothrombin time; tPA, tissue plasminogen activator; PAI-1, plasminogen activator inhibitor-1; TAFI, thrombin activatable fibrinolysis inhibitor; F, factor; VWF, von Willebrand Factor; Rco; IQR, interquartile range; SD, standard deviation; $n$, number.

Multivariable linear regression analysis was used to identify independent determinants of tPA-ROTEM lysis parameters LOT, LT and delta LS, corrected for gender, age. Results are shown in Appendix 7.A Table S7.1. A positive Beta indicates 
the increase in SD of LOT, LT or delta LS if the predictor variable increases with 1 SD. A negative Beta indicates a decrease in SD of LOT, LT or delta LS if the predictor variable increases with 1 SD.

Certain parameters were positively correlated with indicators of enhanced fibrinolysis (defined as a decreased LOT and/or LT or an increased delta LS 100-85 and/or delta LS 85-10) and other parameters were negatively correlated with indicators of enhanced fibrinolysis. Hence, lower levels of fibrinogen, TAFI, a2-AP, prothrombin, FXIII, and leukocytes and higher levels of plasminogen were independently associated with hyperfibrinolysis in these subjects.

Platelet count and TPA activity did not have an effect on TPA-ROTEM lysis parameters. Changes in TPA-ROTEM lysis parameters were in agreement with the expected changes in fibrinolytic proteins associated with hypo- or hyperfibrinolytic profiles as depicted in Figure 7.2.

\section{Results of CIT patients compared to healthy individuals}

The tPA-ROTEM results of 40 healthy individuals ${ }^{13}$ and $72 \mathrm{CIT}$ patients before transfusion are shown in Figure 7.3. All data are presented in Appendix 7.A Table S7.2. Clotting time (CT) and clot formation time (CFT) were significantly longer in CIT patients compared to healthy individuals (CT 58 vs. 51 seconds, $p=0.015$; CFT: 153 vs. 63 seconds, $p<0.001$ ) and maximum clot firmness (MCF) was significantly lower (amplitude 25 vs. $59 \mathrm{~mm}, p<0.001$ ). LOT and LT were shorter in CIT patients compared to healthy individuals (LOT 1495 vs. 2167 seconds, $p<0.001$; LT 2551 vs. 3154 seconds, $p<0.001$ ). Delta LS 85-10 was not significantly different between the two groups, but delta LS 100-85 was significantly higher in the CIT patient group (1.4 vs. $0.9 \% / \mathrm{min}$, $p<0.001)$.

\section{Results of CIT patients with and without a hyperfibrinolytic profile}

According to LOT results, $27 / 66$ (44\%) of the CIT patients were defined as having a hyperfibrinolytic profile, compared to $26 / 65$ (40\%) according to LT results. When LOT and LT results were combined 26/66 (39\%) of patients were defined as having a hyperfibrinolytic profile. According to delta LS 100-85, 24/65 (37\%) had a hyperfibrinolytic profile (data are presented in Appendix 7.A Table S7.3. There were no patients with a hypofibrinolytic profile. 
Figure 7.3 tPA-ROTEM results of healthy individuals compared to CIT patients.
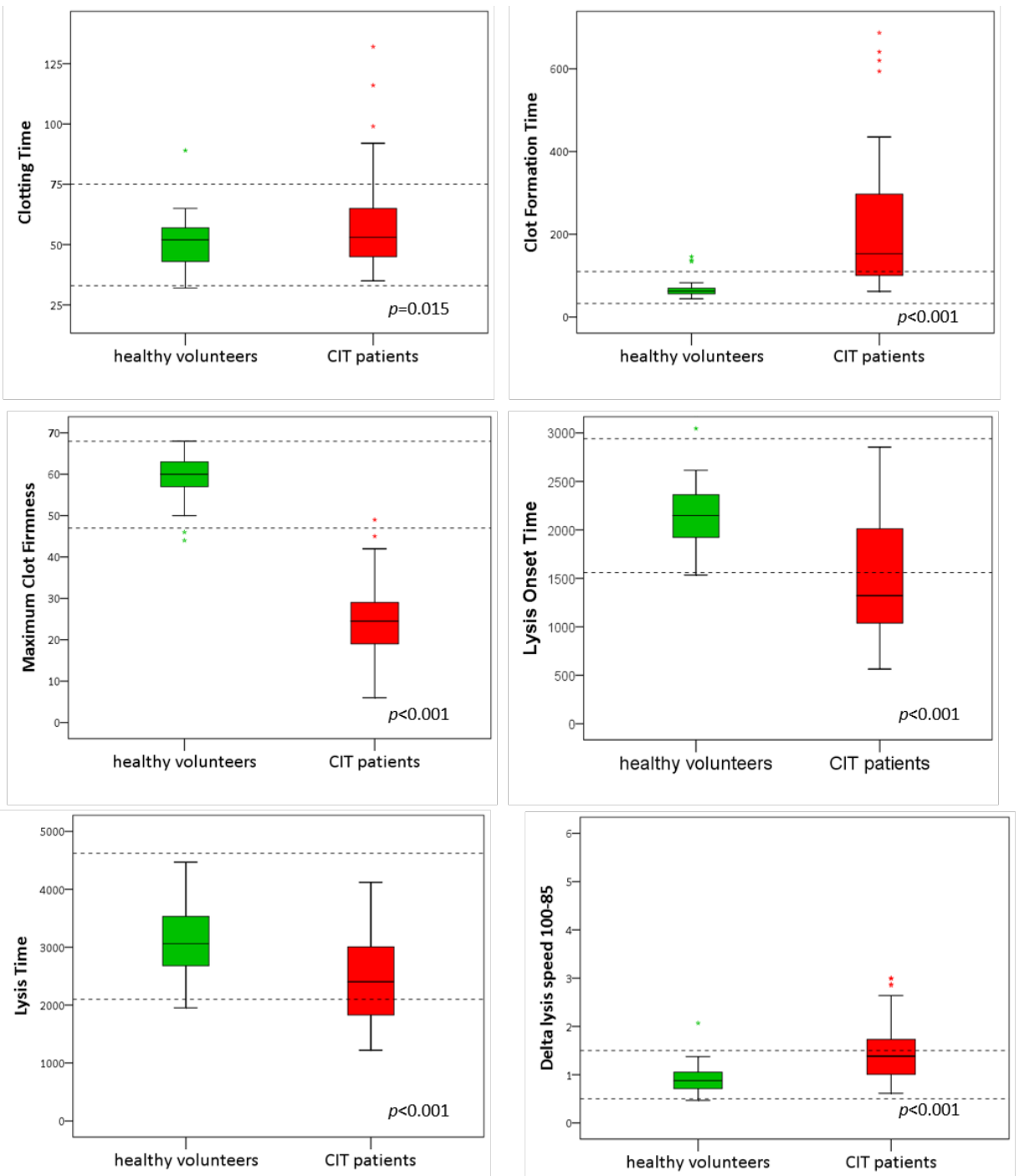

tPA-ROTEM results of healthy individuals $(n=40)$ compared to CIT patients before transfusion $(n=72)$ presented in boxplots, showing the median (dark line in the middle), 1st and 3th quartile, minimum and maximum values and outliers. Levels of significance are given $(p)$, alpha level of significance $=p<0.017$. Dotted lines indicate upper and lower reference value. $n$, number.

Characteristics of CIT patients with and without hyperfibrinolytic profiles (based on abnormal LT ànd LOT) are shown in Table 7.2. Patients without hyperfibrinolytic profiles had lower hemoglobin levels, but higher a2-antiplasmin, FVIII and fibrinogen 
levels compared to the patients with a hyperfibrinolytic profile. No differences in diagnosis of treatment were seen between the two groups.

\section{Results of CIT patients after platelet transfusion}

Table 7.3 shows platelet transfusion characteristics. A total of 65 platelet transfusions (median storage time 5 days, mean platelet count: $347 \times 10^{9} / \mathrm{L}$ ) were given in 62/72 (85\%) patients. Corrected count increment after platelet transfusion was sufficient (>7.5 after 1 hour and $>4.5$ after 24 hours). In 45 patients, results of tPA-ROTEM measurements before, 1 hour and 24 hours after platelet transfusion were available and analyzed. Results are shown in Figure 7.4. (Data are presented in Appendix 7.A Table S7.4).

Table 7.3 Transfusion characteristics.

\begin{tabular}{lc}
\hline Transfusion characteristics & Mean \pm SD, Median [IQR], $\mathbf{n}(\%)$ \\
\hline Number of transfusions given & 65 \\
Patients receiving transfusion & $62(86)$ \\
Time after previous transfusion (days) & $3 \pm 1$ \\
Number of thrombocytes transfused $\left(\times 10^{9} / \mathrm{L}\right)$ & $347 \pm 50$ \\
Storage time (days) & $5[3-6]$ \\
Evaluations 1 hour after transfusion $(\mathrm{n})$ & 57 \\
Evaluations 24 hours after transfusion $(\mathrm{n})$ & 45 \\
Platelet count before transfusion $\left(\times 10^{9} / \mathrm{L}\right)$ & $6[4-9]$ \\
Platelet count 1 hour after transfusion $\left(\times 10^{9} / \mathrm{L}\right)$ & $32[22-43]$ \\
Platelet count 24 hours after transfusion $\left(\times 10^{9} / \mathrm{L}\right)$ & $19[14-26]$ \\
$\mathrm{CCl}$ after 1 hour & $15[11-18]$ \\
$\mathrm{CCl}$ after 24 hours & $8[5-11]$ \\
\hline
\end{tabular}

$\mathrm{CCl}$, corrected count increment; IQR, interquartile range; L, liter; $\mathrm{n}$, number; SD, standard deviation

CT did not change after platelet transfusion (before: 55 seconds vs. 1 hour: 58 seconds vs. 24 hours: 59 seconds, $p=0.215$ ). CFT was significantly shorter 1 hour after platelet transfusion, compared to before platelet transfusion (120 vs. 152 seconds, $p<0.001)$. This effect remained significant after 24 hours. MCF was significantly higher 1 hour after platelet transfusion, compared to before platelet transfusion (39 vs. $23 \mathrm{~mm}, p<0.001$ ) and this effect also remained significant after 24 hours. LOT and LT significantly prolonged 1 hour after transfusion compared to before transfusion (LOT 1785 vs. 1457 seconds, $p<0.001$; LT 2672 vs. 2391 seconds, $p<0.001$ ). Only the LOT remained significantly increased 24 hours after platelet transfusion. Delta LS 100-85 significantly decreased 1 hour after transfusion, compared to before transfusion ( 1.1 vs. $1.4 \% . \mathrm{min}, p=0.009$ ), this effect disappeared after 24 hours. There was no effect of platelet transfusion on delta LS 85-10. 
Figure 7.4 tPA-ROTEM results of CIT patients before, 1 hour after and 24 hours after transfusion.
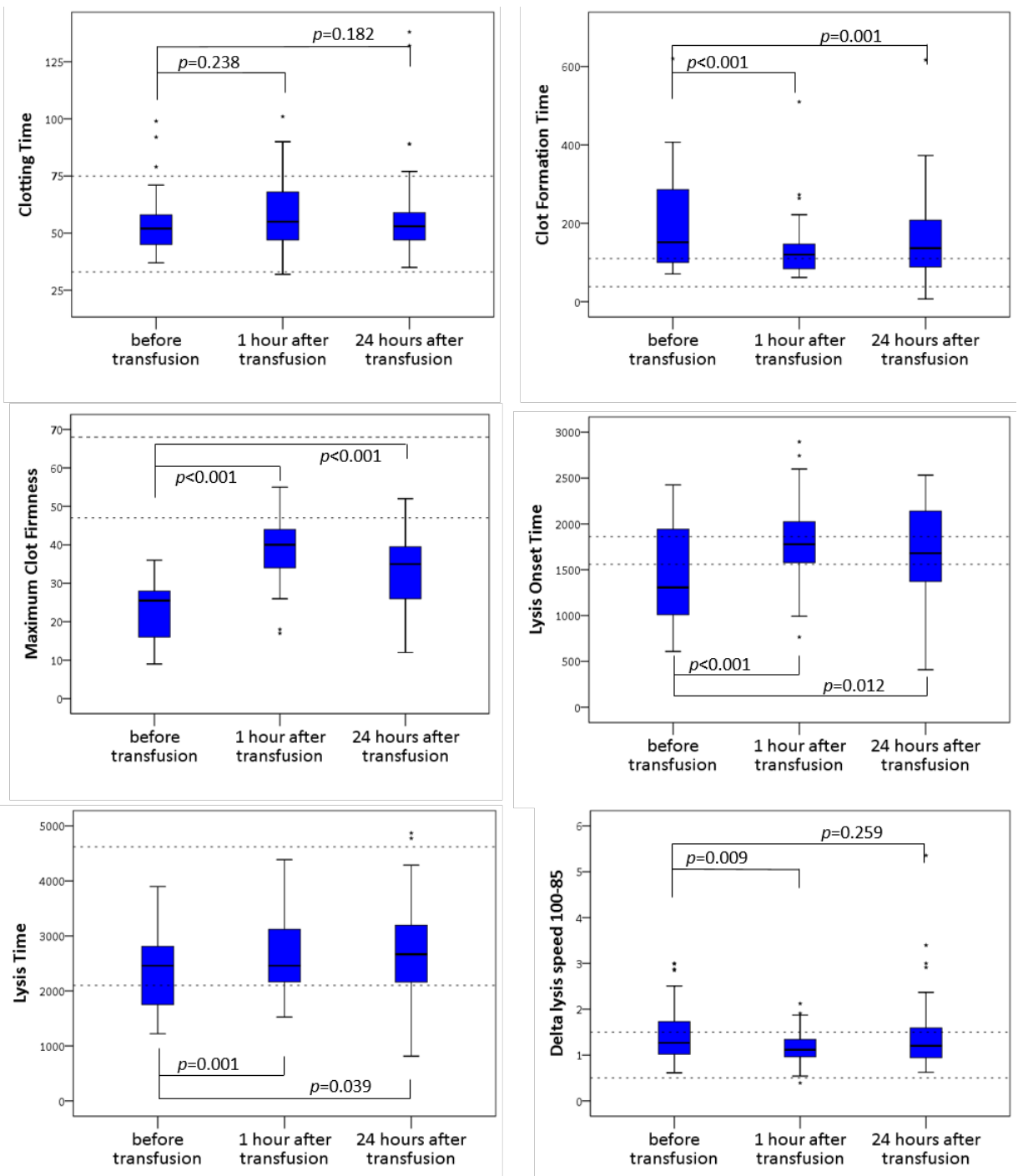

tPA-ROTEM results of CIT patients before, 1 hour after and 24 hours after transfusion $(n=45)$ presented in boxplots, showing the median (dark line in the middle), $1^{\text {st }}$ and 3 th quartile, minimum and maximum values and outliers. Levels of significance are given $(p)$, alpha level of significance $=p<0.017$. Dotted lines indicate upper and lower reference value. $n$, number

One hour after transfusion, significantly less patients had a hyperfibrinolytic profile as compared to before transfusion, according to LOT results ( $23 \%$ vs. $57 \%, p=0.001$ ) and LOT \& LT results combined ( $19 \%$ vs. $38 \%, p=0.012)$. There was a trend toward less 
patients with hyperfibrinolytic profiles according to LT results ( $22 \%$ vs. $41 \%, p=0.039)$. 24 hours after transfusion the number of patients with a hyperfibrinolytic profile increased again (data are presented in Appendix 7.A Table S7.5).

There was no significant difference in number of patient with a hyperfibrinolytic profile according to delta lysis speed 100-85 before and after platelet transfusion. Figure 7.5 shows the direction of change of LOT and LT before and after transfusion. Blue lines indicate an increase in LOT or LT (reflecting a less hyperfibrinolytic profile) after transfusion, red lines indicate a decrease in LOT or LT (reflecting a more hyperfibrinolytic profile) after transfusion. This figure shows that in most patients tPAROTEM changed from a hyperfibrinolytic profile to a less hyperfibrinolytic or normal profile after transfusion. However in some patients tPA-ROTEM changed from a normal to a hyperfibrinolytic profile after transfusion.

Figure 7.5 Direction of change of LOT and LT before and after transfusion.
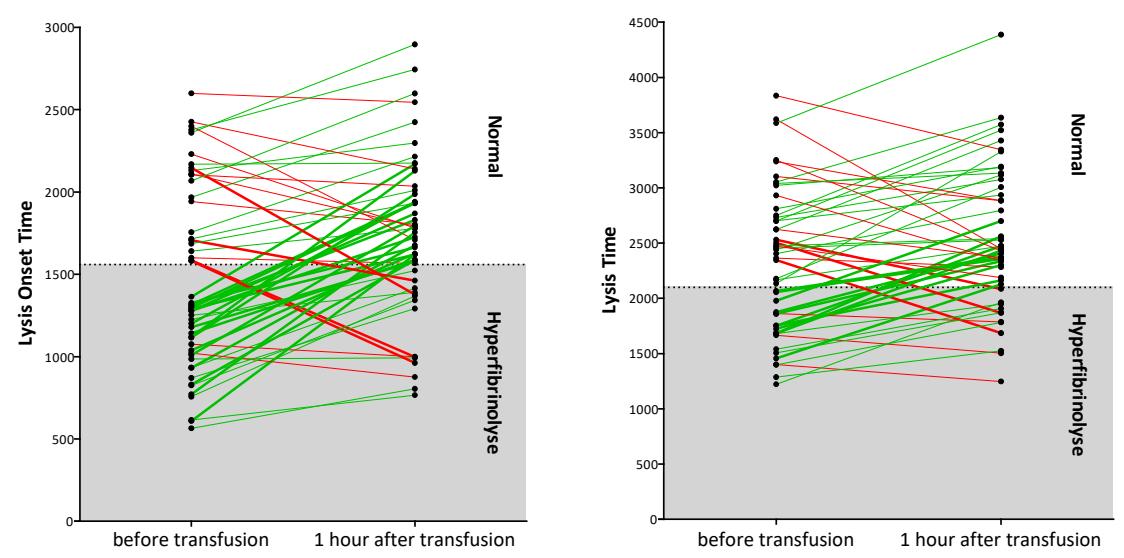

Blue lines indicate an increase in lysis onset time in seconds (left panel) or lysis time in seconds (right panel), reflecting a less hyperfibrinolytic profile, after transfusion, red lines indicate a decrease in LOT or LT (reflecting a more hyperfibrinolytic profile) after transfusion. Bold lines indicate a change in fibrinolytic profile after platelet transfusion. CIT, chemotherapy induced thrombocytopenia.

In patients with a hyperfibrinolytic profile at baseline, the proportion of patients with an increase in LOT and/or LT after transfusion was significantly larger compared to the proportion of patients with a decrease after transfusion (LOT: $93 \%$ vs. $7 \%$ $p<0.001$; LT: $85 \%$ vs. $15 \%, p=0.002$ ). In patients with a normal profile at baseline the proportion of patients with an increase in LOT and/or LT compared to a decrease in LOT and/or LT was not significantly different (data are presented in Appendix 7.A Table S7.6). 
Baseline LOT was negatively correlated with the difference in LOT before and after transfusion (rho=-0.52, $p<0.001$ ), meaning that a lower LOT correlated with a larger difference in LOT before and after transfusion. Also, LT before transfusion was negatively correlated with the difference in LT before and after transfusion (rho=-0.34, $p=0.018$ ). The median change in LOT after transfusion was -48 seconds in the patients with a normal profile, and 520 seconds in the patients with a hyperfibrinolytic profile. This was a significant difference $(p<0.001)$. The median change in LT after transfusion was 93 seconds in the patients with a normal profile, and 397 seconds in the patients with a hyperfibrinolytic profile. This was a near significant difference $(p=0.051)$. Data are presented in Appendix 7.A Table S7.7.

Differences in fibrinolytic protein levels before and after platelet transfusion were analysed. Data are presented in Appendix 7.A Table S7.8. There was a significant difference in fibrinogen level before versus 1 hour after platelet transfusion $(4.7 \mathrm{~g} / \mathrm{dl}$ vs. $4.5 \mathrm{~g} / \mathrm{dl}, p=0.001$ ), and a significant difference in FXIII level before and 1 hour after platelet transfusion ( $104 \%$ vs. $108 \%, p=0.047$ ) However, the differences were rather small and were more likely to reflect the within and between assay variation, than an actual difference due to the presence of factor FXIII in the plasma component (300 $\mathrm{ml})$ of the platelet product, or due to dilution of blood caused by the plasma component of the platelet product.

\section{Discussion}

This is the first study to investigate the use of tPA-ROTEM in CIT patients. Our results showed that the lysis parameters LOT, LT and delta LS are in agreement with the expected changes in fibrinolytic proteins. The TPA-ROTEM is rapid and easy to handle and, in contrast to plasma-based tests, uses whole blood, allowing to assess all of the cellular components that may play a major role in clot formation and fibrinolysis. By use of the TPA-ROTEM, we found that CIT patients have slower clot formation and form weaker clots, which are more susceptible to tPA induced fibrinolysis, as compared to healthy individuals.

Platelet transfusions significantly increased resistance to fibrinolysis, with the most distinct effect in patients with a hyperfibrinolytic profile at baseline. Previous studies have shown that platelets influence fibrin formation and clot structure by: (i) regulation of local thrombin generation through exposure of the negatively charged phospholipid phosphatidylserine (PS) at the platelet membrane surface ${ }^{24}$, (ii) release of polyphosphate from dense granules, which directly influences fibrin architecture and attenuates fibrinolysis through reduced binding of fibrinolytic proteins ${ }^{25}$, (iii) 
release of platelet factor 4 which reduces fibrin network porosity ${ }^{26}$, (iv) release of platelet factor XIII which increases the fibrinolytic resistance of platelet rich clots by catalyzing the crosslinking of alpha 2-antiplasmin to fibrin ${ }^{27}$, (v) promoting the formation of a dense fibrin network, highly resistant to tPA-activated fibrinolysis, through platelet glycoprotein IIb/III ${ }^{28}$.

In CIT patients, all these interactions and mechanisms are likely to be altered, leading to weaker clots, and increasing susceptibility to clot lysis. Even more, these alterations might not only be due to the low platelet count, but also due to altered platelet function. Baaten et al. found lower platelet responsiveness in CIT patients, due to mitochondrial dysfunction, possibly caused by chemotherapy effect on the megakaryocytes ${ }^{29}$.

Even after platelet transfusions, tPA-ROTEM profiles remained hyperfibrinolytic in some patients. Even more, within the group of CIT patients before transfusion, large variability was seen in lysis parameter results and we could define two subgroups: patients with a hyperfibrinolytic profile and patients without a hyperfibrinolytic profile. This indicates that besides platelets, other factors influence clot susceptibility to fibrinolysis in these patients. Overall, CIT patients had high fibrinogen, D-dimer, FVIII and VWF levels which might be a reflection of endothelial activation and a procoagulant state ${ }^{30-32}$

We also found that CIT patients had low TAFI levels. This might contribute to an increased susceptibility to fibrinolysis. TAFI is important for the protection against fibrinolysis especially at the site of clot formation. Even though TAFI in platelets represents only $0.1 \%$ of the total TAFI in blood, secretion of platelet derived TAFI has a substantial effect on the fibrinolytic cascade in addition to TAFI present in plasma ${ }^{33}$. Release of TAFI from activated platelets concentrated within the fibrin clot leads to significant concentrations and variations in TAFI concentration were found to influence clot lysis time $\mathrm{e}^{34,35}$ and thromboelastographic lysis parameters ${ }^{36}$. In thrombocytopenia, local TAFI concentrations within the clot might lead to less protection against fibrinolysis ${ }^{9}$. Why CIT patients have low TAFI levels is not clear, research results of TAFI in cancer are conflicting, some studies show high levels of TAFI in cancer patients while other studies show that TAFI levels in cancer patients are not different as compared to a control group ${ }^{37-40}$.

The low leukocyte count in CIT patients might also lead to more susceptibility to clot lysis. Leukocytes influence coagulation by expressing tissue factor (TF) and the release of pro-coagulant molecules. In this way, both monocytes and neutrophils can modulate activity of the fibrinolytic pathway and susceptibility to fibrinolysis. In an in vitro model of clot lysis, Semeraro et al. found that activated monocytes inhibited fibrinolysis, through a tissue factor mediated way ${ }^{41}$. Also, an increase in TF-expressing 
circulating monocytes has been documented in pathologic conditions like antiphospholipid syndrome and thrombosis ${ }^{42,43}$. Low leukocyte count in CIT patients may lead to less TF expression and less inhibition of fibrinolysis.

When we compared the subgroups of patients with and without a hyperfibrinolytic profile, we found that patients who did not have a hyperfibrinolytic profile had higher fibrinogen, higher FVIII and higher $\alpha 2$-AP levels. Lang et al. showed that cloth strength measured by ROTEM in thrombocytopenia increases in a fibrinogen dependent manner and increasing fibrinogen levels increased cloth strength even at low platelet count in vitro ${ }^{10}$. Shenkman et al. showed that spiking thrombocytopenic blood with fibrinogen increased clot strength but did not influence tPA-induced fibrinolysis ${ }^{9}$. Another study showed that higher fibrinogen levels compensate the decrease in clot firmness observed in thrombocytopenic patients following cardiopulmonary bypass and reduce bleeding and transfusion needs ${ }^{44}$.

Also FVIII is important for resistance to fibrinolysis. In hemophilia patients lack of FVIII results in the production of a thicker but brittle fiber network with fibers that are more sensitive to fibrinolysis ${ }^{45,46} . \alpha 2-\mathrm{AP}$ is a potent regulator of fibrinolysis and previous studies showed that elevated levels of $\alpha 2-A P$ is associated with failure of tissue plasminogen activator (tPA) reperfusion therapy in patients with pulmonary embolism and stroke ${ }^{47-49}$. In CIT patients, high fibrinogen, FVIII and $\alpha 2$-AP levels might act as a compensatory mechanism to maintain clot firmness even in the absence of platelets.

This study has some limitations. Although the tPA-ROTEM can be considered a global hemostatic assay, the role of the endothelial damage and/or activation cannot be studied. Furthermore, clot structure itself was not assessed and we can merely speculate on the role of platelets and other coagulation factors in how clot structure might be altered in CIT patients. Further investigation of these clots with electronic microscopy or in vitro clot formation (microfluidic tests) might give new insights. Also, we were not able to measure platelet content of fibrinolytic proteins like TAFI and PAI-1 and could not compare this between healthy volunteers and CIT patients. Finally, bleeding episodes were not registered in our study.

Large multicenter, randomized controlled studies in CIT patients could elucidate the clinical relevance of a hyperfibrinolytic tPA-ROTEM profile on bleeding. Future research could focus on including parameters like tPA-ROTEM LOT, LT and delta LS, fibrinogen, FVIII and $\alpha 2-A P$ in the decision to transfuse platelets of a single platelet count and could test the hypothesis that only patients with a hyperfibrinolytic profile benefit from a platelet transfusion. Also the effect of antifibrinolytic therapy should be further investigated. 


\section{References}

1. Chapin JC, Hajjar KA. Fibrinolysis and the control of blood coagulation. Blood Reviews 2015;29(1): 17-24.

2. Stanworth SJ, et al. A no-prophylaxis platelet-transfusion strategy for hematologic cancers. N Engl J Med 2013;368(19):1771-80.

3. Uhl L, et al. Laboratory predictors of bleeding and the effect of platelet and RBC transfusions on bleeding outcomes in the PLADO trial. Blood 2017;130(10):1247-58.

4. Wandt $\mathrm{H}$, et al. Therapeutic platelet transfusion versus routine prophylactic transfusion in patients with haematological malignancies: an open-label, multicentre, randomised study. Lancet 2012; 380(9850):1309-16.

5. Vinholt PJ, et al. Prediction of bleeding and prophylactic platelet transfusions in cancer patients with thrombocytopenia. Platelets 2016;27(6):547-54.

6. Estcourt LJ, et al. Prospective observational cohort study of the association between thromboelastometry, coagulation and platelet parameters and bleeding in patients with haematological malignancies- The ATHENA study. Br J Haematol 2014;166(4):581-91.

7. Friedmann $\mathrm{AM}$, et al. Do basic laboratory tests or clinical observations predict bleeding in thrombocytopenic oncology patients? A reevaluation of prophylactic platelet transfusions. Transfus Med Rev 2002;16(1):34-45.

8. Shenkman B, et al. In vitro evaluation of clot quality and stability in a model of severe thrombocytopenia: effect of fibrinogen, factor XIII and thrombin-activatable fibrinolysis inhibitor. Blood Transfus 2014;12(1):78-84.

9. Shenkman B, et al. Rotation thromboelastometry analysis of clot formation and fibrinolysis in severe thrombocytopenia: effect of fibrinogen, activated prothrombin complex concentrate, and thrombinactivatable fibrinolysis inhibitor. Int J Lab Hematol 2015;37(4):521-9.

10. Lang $\mathrm{T}$, et al. The Effects of Fibrinogen Levels on Thromboelastometric Variables in the Presence of Thrombocytopenia. Anesth Analg 2009;108(3):751-8.

11. Munk-Andersen $\mathrm{H}$, et al. Fibrinogen concentrate improves clot strength in patients with haematological malignancies requiring platelet transfusion. Transfus Med 2016;26(4):291-6.

12. Troppan KT, et al. The clinical significance of fibrinogen plasma levels in patients with diffuse large $B$ cell lymphoma. J Clin Pathol 2016;69(4):326-30.

13. Mei $Y$, et al. Plasma fibrinogen level may be a possible marker for the clinical response and prognosis of patients with breast cancer receiving neoadjuvant chemotherapy. Tumor Biology 2017;39(6): 1010428317700002.

14. Kowalski E, Kopec M, Niewiarowski. An evaluation of the euglobulin method for the determination of fibrinolysis. J Clin Pathol 1959;12(3):215-8.

15. Kuiper GJAJM, et al. Validation of a modified thromboelastometry approach to detect changes in fibrinolytic activity. Thrombosis Journal 2016;14:1.

16. Rijken DC, et al. Evidence for an enhanced fibrinolytic capacity in cirrhosis as measured with two different global fibrinolysis tests. Journal of Thrombosis and Haemostasis, 2012. 10(10): p. 2116-2122.

17. Hoekstra, J., et al., Impaired fibrinolysis as a risk factor for Budd-Chiari syndrome. Blood 2010;115(2): 388-95.

18. Meltzer ME, et al. The impact of the fibrinolytic system on the risk of venous and arterial thrombosis. Semin Thromb Hemost 2009;35(5):468-77.

19. Meltzer ME, et al. Synergistic effects of hypofibrinolysis and genetic and acquired risk factors on the risk of a first venous thrombosis. Plos Medicine 2008;5(5):751-9.

20. De Wee EM, et al. Effect of fibrinolysis on bleeding phenotype in moderate and severe von Willebrand disease. Haemophilia 2012;18(3):444-51.

21. Dekker SE, et al. Lysis onset time as diagnostic rotational thromboelastometry parameter for fast detection of hyperfibrinolysis. Anesthesiology 2014;121(1):89-97.

22. Schochl H, et al. Hyperfibrinolysis After Major Trauma: Differential Diagnosis of Lysis Patterns and Prognostic Value of Thrombelastometry. J Trauma 2009; 67(1):125-31. 
23. Nielsen VG, Cohen BM, Cohen E. Elastic modulus-based thrombelastographic quantification of plasma clot fibrinolysis with progressive plasminogen activation. Blood Coagul Fibrinolysis 2006;17(1):75-81.

24. Swieringa $F$, et al. Integrating platelet and coagulation activation in fibrin clot formation. Res Pract Thromb Haemost 2018;2(3):450-60.

25. Mutch NJ, et al. Polyphosphate modifies the fibrin network and down-regulates fibrinolysis by attenuating binding of tPA and plasminogen to fibrin. Blood 2010;115(19):3980-8.

26. Wolberg AS. Plasma and cellular contributions to fibrin network formation, structure and stability. Haemophilia 2010;16 Suppl 3:7-12.

27. Reed GL, Matsueda GR, Haber E. Platelet factor XIII increases the fibrinolytic resistance of platelet-rich clots by accelerating the crosslinking of alpha 2-antiplasmin to fibrin. Thromb Haemost 1992;68(3): 315-20.

28. Collet JP, et al. Disaggregation of in vitro preformed platelet-rich clots by abciximab increases fibrin exposure and promotes fibrinolysis. Arterioscler Thromb Vasc Biol 2001;21(1):142-8.

29. Baaten CCFMJ, et al. Impaired mitochondrial activity explains platelet dysfunction in thrombocytopenic cancer patients undergoing chemotherapy. Haematologica 2018;103(9):1557-67.

30. Minnema MC, et al. Extremely high levels of von Willebrand factor antigen and of procoagulant factor VIII found in multiple myeloma patients are associated with activity status but not with thalidomide treatment. J Thromb Haemost 2003;1(3):445-9.

31. van Marion AM, et al. Prospective evaluation of coagulopathy in multiple myeloma patients before, during and after various chemotherapeutic regimens. Leuk Res 2008;32(7):1078-84.

32. Levi M. Cancer-related coagulopathies. Thromb Res 2014;133 Suppl 2:S70-5.

33. Schadinger SL, et al. Secretion and antifibrinolytic function of thrombin-activatable fibrinolysis inhibitor from human platelets. J Thromb Haemost 2010;8(11):2523-9.

34. Mosnier LO, et al. Plasma TAFI levels influence the clot lysis time in healthy individuals in the presence of an intact intrinsic pathway of coagulation. Thromb Haemost 1998;80(5):829-35.

35. Mosnier LO, et al. Identification of thrombin activatable fibrinolysis inhibitor (TAFI) in human platelets. Blood 2003;101(12):4844-6.

36. Carrieri $\mathrm{C}$, et al. The role of thrombin activatable fibrinolysis inhibitor and factor $\mathrm{XI}$ in plateletmediated fibrinolysis resistance: a thromboelastographic study in whole blood. J Thromb Haemost 2011;9(1):154-62.

37. Radu CM, et al. Thrombin Activatable Fibrinolysis inhibitor in Cancer Patients with and without Venous Thromboembolism. Thromb Res 2013;132(4):484-6.

38. Balcik OS, et al. Serum thrombin activatable fibrinolysis inhibitor levels in patients with newly diagnosed multiple myeloma. Blood Coagul Fibrinolysis 2011;22(4):260-3.

39. Hataji $O$, et al. Increased circulating levels of thrombin-activatable fibrinolysis inhibitor in lung cancer patients. Am J Hematol 2004;76(3):214-9.

40. Eser $\mathrm{M}$, et al. Is there any role of thrombin activatable fibrinolysis inhibitor in the development of a hypercoagulable state in gastric cancer. World J Surg Oncol 2012;10(1):180.

41. Semeraro F, et al. Tissue factor-expressing monocytes inhibit fibrinolysis through a TAFI-mediated mechanism, and make clots resistant to heparins. Haematologica 2009;94(6):819-26.

42. Swystun LL, Liaw PC. The role of leukocytes in thrombosis. Blood, 2016. 128(6): p. 753-62.

43. Cuadrado, M.J., et al., Thrombosis in primary antiphospholipid syndrome: a pivotal role for monocyte tissue factor expression. Arthritis Rheum 1997;40(5):834-41.

44. Ranucci $M$, et al. Fibrinogen levels compensation of thrombocytopenia-induced bleeding following cardiac surgery. Int J Cardiol 2017;249:96-100.

45. He S, et al. The role of recombinant factor VIIa (FVIIa) in fibrin structure in the absence of FVIII/FIX. J Thromb Haemost 2003;1(6):1215-9.

46. Lisman $\mathrm{T}$, et al. Inhibition of fibrinolysis by recombinant factor VIla in plasma from patients with severe hemophilia A. Blood 2002;99(1):175-9.

47. Reed GL, et al. alpha2-Antiplasmin: New Insights and Opportunities for Ischemic Stroke. Semin Thromb Hemost 2017;43(2):191-9.

48. Marti-Fabregas J, et al. Hemostatic markers of recanalization in patients with ischemic stroke treated with rt-PA. Neurology 2005;65(3):366-70. 
Chapter 7

49. Butte $\mathrm{AN}$, et al. Alpha 2-antiplasmin causes thrombi to resist fibrinolysis induced by tissue plasminogen activator in experimental pulmonary embolism. Circulation 1997;95(7):1886-91. 


\section{Appendix 7.A Supplementary materials}

Table S7.1 Determinants of tPA-ROTEM in CIT patients ( $n=72)$.

\begin{tabular}{|c|c|c|c|c|c|c|c|c|}
\hline & \multicolumn{2}{|c|}{$\begin{array}{c}\text { Lysis Onset Time } \\
\mathbf{R}^{2}=47 \%\end{array}$} & \multicolumn{2}{|c|}{$\begin{array}{l}\text { Lysis Time } \\
\mathbf{R}^{2}=46 \%\end{array}$} & \multicolumn{2}{|c|}{$\begin{array}{c}\text { Delta LS } 85-10 \\
R^{2}=52 \%\end{array}$} & \multicolumn{2}{|c|}{$\begin{array}{c}\text { Delta LS } 100-85 \\
R^{2}=43 \%\end{array}$} \\
\hline & Beta & $p$ & Beta & $p$ & Beta & $p$ & Beta & $p$ \\
\hline Hemoglobin & -0.147 & 0.223 & -0.211 & 0.086 & 0.275 & 0.021 & 0.073 & 0.477 \\
\hline Thrombocytes & 0.076 & 0.546 & 0.030 & 0.817 & 0.088 & 0.473 & -0.093 & 0.394 \\
\hline Leukocytes & 0.301 & 0.027 & 0.283 & 0.040 & -0.062 & 0.632 & -0.197 & 0.091 \\
\hline Fibrinogen & 0.07 & 0.640 & 0.23 & 0.137 & -0.507 & 0.001 & -0.376 & 0.006 \\
\hline Plasminogen & -0.45 & 0.014 & -0.36 & 0.047 & 0.197 & 0.253 & 0.345 & 0.013 \\
\hline a2-AP & 0.37 & 0.023 & 0.44 & 0.010 & -0.224 & 0.153 & -0.255 & 0.061 \\
\hline tPA activity & -0.13 & 0.381 & -0.00 & 0.989 & -0.058 & 0.681 & 0.069 & 0.570 \\
\hline PAI-1 activity & 0.26 & 0.061 & 0.17 & 0.225 & 0.101 & 0.453 & -0.131 & 0.281 \\
\hline TAFI & 0.18 & 0.194 & 0.15 & 0.288 & -0.017 & 0.889 & -0.270 & 0.034 \\
\hline FII & 0.38 & 0.023 & 0.27 & 0.108 & -0.047 & 0.762 & -0.224 & 0.075 \\
\hline FXIII & 0.32 & 0.008 & 0.14 & 0.256 & 0.098 & 0.403 & -0.190 & 0.074 \\
\hline
\end{tabular}

Multivariable linear regression was used to identify independent determinants of the tPA-ROTEM lysis parameters LOT, LT and delta LS, corrected for age and gender. A positive Beta indicates the increase in SD of LOT or LT if the predictor variable increases with 1 SD. A negative Beta indicates a decrease in SD of LOT or LT. a2-AP, alfa-2-antiplasmin; tPA, tissue plasminogen activator; PAI-1, plasminogen activator inhibitor-1; TAFI, thrombin activatable fibrinolysis inhibitor; $F$, factor. Beta = standardised regression coefficients. $R^{2}=$ the percentage of the response variable variation that is explained by the regression model.

Table S7.2 tPA-ROTEM results in healthy individuals $(n=40)$ compared to CIT patients $(n=72)$.

\begin{tabular}{lccc}
\hline tPA-ROTEM parameter & Healthy individuals & CIT patients & p \\
\hline CT (s) & $51 \pm 11$ & $58 \pm 18$ & 0.015 \\
CFT (s) & $63[56-70]$ & $153[100-299]$ & $<0.001$ \\
MCF (mm) & $59 \pm 5$ & $25 \pm 9$ & $<0.001$ \\
MCFt (s) & $1092 \pm 134$ & $820 \pm 354$ & $<0.001$ \\
alpha (dgr) & $77 \pm 4$ & $69 \pm 7$ & $<0.001$ \\
LOT (s) & $2167 \pm 355$ & $1495 \pm 577$ & $<0.001$ \\
LT (s) & $3154 \pm 663$ & $2551 \pm 634$ & $<0.001$ \\
LOT-MCFt (s) & $1075 \pm 290$ & $713 \pm 290$ & $<0.001$ \\
Delta LS 100-85 (\%/min) & $0.9[0.7-1.1]$ & $1.4[1.0-1.8]$ & $<0.001$ \\
Delta LS 85-10 (\%/min) & $4.9[3.8-5.7]$ & $4.9[3.9-6.2]$ & 0.669 \\
\hline
\end{tabular}

Data are presented in mean $\pm S D$ or median $[I Q R]$, alpha level of significance $=p<0.05$. CT, clotting time; CFT, clot formation time; MCF, maximum clot firmness; MCFt, time to maximum clot firmness; LOT, Iysis onset time; LS, lysis speed; LT, lysis time. 
Table S7.3 Number of CIT patients with hyperfibrinolysis according to tPA-ROTEM results ( $n=72)$.

\begin{tabular}{lcc}
\hline tPA-ROTEM parameter & Profile & $\mathbf{n}(\%)$ \\
\hline LOT & Hyperfibrinolytic* & $27(44)$ \\
& Normal & $29(56)$ \\
& Total* & $66(100)$ \\
LT & Hyperfibrinolytic* & $26(40)$ \\
& Normal & $39(60)$ \\
LOT \& LT & Total** & $65(100)$ \\
& Hyperfibrinolytic* & $26(39)$ \\
& Normal & $40(61)$ \\
Delta LS 100-85 & Total** & $66(100)$ \\
& Hyperfibrinolytic* & $24(37)$ \\
& Normal & $41(63)$ \\
\hline
\end{tabular}

* Hyperfibrinolysis was defined when LOT was $<1560$ s., LT was $<2100$ s. or Delta LS $100-85$ was $>1.5 \% / \mathrm{min}$

** in some cases LOT or LT was not measured, therefor numbers do not count up to the total number of patients. LOT, lysis onset time; LS, lysis speed; LT, lysis time.

Table S7.4 tPA-ROTEM results in CIT patients before, 1 hour and 24 hours after transfusion ( $n=45)$.

\begin{tabular}{|c|c|c|c|c|c|c|c|}
\hline $\begin{array}{l}\text { tPA ROTEM } \\
\text { parameter }\end{array}$ & $\begin{array}{c}\text { before } \\
\text { transfusion }\end{array}$ & $\begin{array}{l}1 \text { hour after } \\
\text { transfusion }\end{array}$ & $\begin{array}{c}24 \text { hours after } \\
\text { transfusion }\end{array}$ & $\begin{array}{c}p \text { all } \\
\text { groups }\end{array}$ & $\begin{array}{l}p \text { before } \\
\text { vs. } 1 \text { hour }\end{array}$ & $\begin{array}{c}p \text { before vs. } \\
24 \text { hours }\end{array}$ & $\begin{array}{l}p 1 \text { hour vs. } \\
24 \text { hours }\end{array}$ \\
\hline $\mathrm{CT}(\mathrm{s})$ & $55 \pm 14$ & $58 \pm 16$ & $59 \pm 21$ & 0.215 & 0.238 & 0.182 & 0.829 \\
\hline $\mathrm{CFT}(\mathrm{s})$ & 152 [98-292] & $120[84-120]$ & $137[87-210]$ & 0.000 & $<0.001$ & 0.001 & 0.008 \\
\hline $\mathrm{MCF}(\mathrm{mm})$ & $23 \pm 7$ & $39 \pm 8$ & $34 \pm 9$ & $<0.001$ & $<0.001$ & $<0.001$ & $<0.001$ \\
\hline alpha (dgr) & $69 \pm 6$ & $71 \pm 5$ & $69 \pm 8$ & 0.152 & 0.063 & 0.659 & 0.238 \\
\hline LOT (s) & $1457 \pm 525$ & $1785 \pm 392$ & $1663 \pm 554$ & $<0.001$ & $<0.001$ & 0.012 & 0.089 \\
\hline $\mathrm{LT}(\mathrm{s})$ & $2391 \pm 676$ & $2672 \pm 628$ & $2649 \pm 904$ & 0.005 & 0.001 & 0.039 & 0.833 \\
\hline Delta LS 85-10 (\%/min) & $4.9[4.0-6.5]$ & $6.1[4.0-7.3]$ & $5.2[3.6-7.1]$ & 0.241 & 0.412 & 0.936 & 0.375 \\
\hline Delta LS 100-85 (\%/min) & $1.4[1.0-1.8]$ & $1.1[1.0-1.5]$ & $1.2[0.9-1.7]$ & 0.500 & 0.009 & 0.259 & 0.077 \\
\hline
\end{tabular}

Data are presented in mean \pm SD or median $[\mathrm{IQR}]$, alpha level of significance $=p<0.017$. CT, clotting time; CFT, clot formation time; MCF, maximum clot firmness; MCFt, time to maximum clot firmness; LOT, lysis onset time; LS, lysis speed; LT, lysis time. 
Table S7.5 Number of CIT patients with hyperfibrinolysis according to tPA-ROTEM results before, 1 hour and 24 hours after platelet transfusion $(n=45)$.

\begin{tabular}{|c|c|c|c|c|c|c|c|c|}
\hline $\begin{array}{l}\text { tPA } \\
\text { ROTEM } \\
\text { parameter }\end{array}$ & profile & $\begin{array}{c}\text { before } \\
\text { transfusion } \\
\text { n (\%) }\end{array}$ & $\begin{array}{c}1 \text { hour } \\
\text { after } \\
\text { transfusion } \\
n(\%)\end{array}$ & $\begin{array}{c}24 \text { hours } \\
\text { after } \\
\text { transfusion } \\
n(\%)\end{array}$ & $p$ & $\begin{array}{c}p \text { before } \\
\text { vs. } \\
1 \text { hour }\end{array}$ & $\begin{array}{l}p \text { before } \\
\text { vs. } \\
24 \text { hours }\end{array}$ & $\begin{array}{c}p 1 \text { hour } \\
\text { vs. } \\
24 \text { hours }\end{array}$ \\
\hline \multirow[t]{3}{*}{ LOT } & Hyperfibrinolytic* & $24(57)$ & $10(23)$ & $16(37)$ & 0.001 & 0.001 & 0.039 & 0.146 \\
\hline & Normal & $18(43)$ & $33(77)$ & $27(63)$ & & & & \\
\hline & Total* & $42(100)$ & $43(100)$ & $43(100)$ & & & & \\
\hline \multirow[t]{3}{*}{ LT } & Hyperfibrinolytic* & $17(41)$ & $9(22)$ & $10(25)$ & 0.001 & 0.039 & 0.070 & 0.687 \\
\hline & Normal & $25(60)$ & $32(78)$ & $31(75)$ & & & & \\
\hline & Total** & $42(100)$ & $41(100)$ & $41(100)$ & & & & \\
\hline \multirow[t]{3}{*}{ LOT \& LT } & Hyperfibrinolytic* & $17(38)$ & $08(19)$ & $10(23)$ & 0.008 & 0.012 & 0.070 & 0.453 \\
\hline & Normal & $25(60)$ & $35(81)$ & $33(77)$ & & & & \\
\hline & Total** & $42(100)$ & $43(100)$ & $43(100)$ & & & & \\
\hline Delta LS & Hyperfibrinolytic* & $14(34)$ & $07(17)$ & $11(26)$ & 0.046 & 0.065 & 0.219 & 0.453 \\
\hline \multirow[t]{2}{*}{$100-85$} & Normal & $27(66)$ & $34(83)$ & $32(74)$ & & & & \\
\hline & Total** & $41(100)$ & $41(100)$ & $43(100)$ & & & & \\
\hline
\end{tabular}

Alpha level of significance $=p<0.017$. ${ }^{*}$ Hyperfibrinolytic profile was defined when LOT was $<1560 \mathrm{~s}$., or LT was $<2100 \mathrm{~s}$, or Delta LS $100-85$ was $>1.5 \% / \mathrm{min} . * *$ in some cases LOT, LT or Delta LS was not measured, therefor numbers do not count up to the total number of patients. LOT, lysis onset time; LS, lysis speed; LT, lysis time

Table S7.6 Differences in direction of change of LOT and LT after transfusion according to baseline profile $(n=57)$.

\begin{tabular}{|c|c|c|c|}
\hline \multirow[t]{2}{*}{ LOT profile at baseline } & \multicolumn{3}{|c|}{ Direction of change of LOT after transfusion } \\
\hline & Increase & Decrease & $p^{*}$ \\
\hline Normal profile & $10(45)$ & $12(55)$ & 0.670 \\
\hline Hyperfibrinolytic profile & $28(93)$ & $2(7)$ & 0.000 \\
\hline \multirow[t]{2}{*}{ LT profile at baseline } & \multicolumn{3}{|c|}{ Direction of change of LT after transfusion } \\
\hline & Increase & Decrease & $p^{*}$ \\
\hline Normal profile & $17(59)$ & $12(41)$ & 0.353 \\
\hline Hyperfibrinolytic profile & $17(85)$ & $3(15)$ & 0.002 \\
\hline
\end{tabular}

${ }^{*}$ Chi-square Data are presented as n (\%). LOT, lysis onset time; LT, lysis time.

Table S7.7 Magnitude of change of LOT and LT according to baseline profile ( $n=57)$.

\begin{tabular}{lccc}
\hline Change after transfusion & Normal profile & $\begin{array}{c}\text { Baseline } \\
\text { Hyperfibrinolytic profile }\end{array}$ & $\boldsymbol{p}^{*}$ \\
\hline LOT in seconds & $-48[-351-323]$ & $520[321-748]$ & 0.000 \\
LT in seconds & $93[-399-631]$ & $397[277-709]$ & 0.051 \\
\hline
\end{tabular}

*Wilcoxon. Data are presented as median [IQR]. LOT, lysis onset time; LT, lysis time. 
Table S7.8 Fibrinolysis proteins in CIT patients before and 1 hour after platelet transfusion ( $n=57)$.

\begin{tabular}{lccc}
\hline & Before transfusion & 1 hour after transfusion & $\boldsymbol{p}$ value \\
\hline Fibrinogen (g/dl) & $4.7 \pm 1.5$ & $4.5 \pm 1.4$ & 0.001 \\
Plasminogen (\%) & $91 \pm 17$ & $90 \pm 16$ & 0.542 \\
$\alpha 2-A P(\%)$ & $102 \pm 16$ & $101 \pm 14$ & 0.169 \\
tPA activity (ng/ml) & $8 \pm 4$ & $7 \pm 5$ & 0.060 \\
PAI-1 activity (ng/ml) & $0.58[0.28-1.02]$ & $0.48[0.25-0.90]$ & $0.056^{*}$ \\
TAFI (\%) & $87 \pm 23$ & $89 \pm 24$ & 0.246 \\
FII (\%) & $91 \pm 14$ & $90 \pm 13$ & 0.775 \\
FXIII (\%) & $104 \pm 28$ & $108 \pm 25$ & 0.047 \\
\hline
\end{tabular}

*Wilcoxon. Data are presented in mean \pm SD or median [IQR], alpha level of significance $=p<0.05$. a2-AP, alfa-2-antiplasmin; tPA, tissue plasminogen activator; PAI-1, plasminogen activator inhibitor-1; TAFI, thrombin activatable fibrinolysis inhibitor; $F$, factor. 



\section{CHAPTER 8}

General discussion and future perspectives 



\section{General discussion of main findings}

The studies in this thesis were performed (i) to evaluate diagnostic tests in patients referred for bleeding evaluation (chapter 2, 3, 4 and 5) and (ii) to gain more insight in mechanisms of bleeding in patients with chemotherapy induced thrombocytopenia (chapter 6 and 7). The results of our studies led to the formation of a number of statements and topics, which will be addressed in this chapter. For part I, two cases to illustrate the difficulties and challenges in the diagnostic work-up of patients with mild bleeding symptoms, are presented. In the end, we will focus on future perspectives for both parts of this thesis.

\section{Defined statements and topics}

- Definition of a mild bleeding disorder: let's keep it simple!

- Diagnosing mild bleeding disorders: clinical utility is in the eyes of the beholder.

- The bleeding assessment tool is of limited additional diagnostic value to the workup of patients referred for bleeding evaluation in daily practice.

- Using the currently available laboratory tests, we might never know why most patients bleed.

- Bleeding risk in patients with mild bleeding disorders: putting together pieces of evidence.

- Balance of hemostasis in hemato-oncology patients: an extra dimension.

\section{Part I. Diagnosis of mild bleeding disorders}

\section{Case 1}

A male patient, 23 years old, was referred by his general practitioner for evaluation of bleeding symptoms. He presented with spontaneous hematomas on his arms and legs with a maximum diameter of $3 \mathrm{~cm}$. Upon questioning he mentioned prolonged bleeding of small wounds (15 minutes) when inflicted with shaving. He never needed dental extraction or surgery, nor did he use any medication, and his further medical history was unremarkable. The calculated bleeding score was 2 (normal). Family history however, revealed von Willebrand disease (VWD) in his sister and mother. After laboratory testing, he was diagnosed with mild VWD type 1 (VWF activity 39\%).

\section{Case 2}

A female patient, 68 years old, was referred for bleeding evaluation before knee replacement surgery of her right knee. Her bleeding history revealed a dental extraction at the age of 21 with prolonged bleeding requiring extra sutures. In the 
past, she had 3 vaginal deliveries, after which she consequently bled for 6 weeks, resulting in iron deficiency requiring iron therapy. After colonic biopsy she presented to the emergency room with rectal bleeding, which was self-limiting. At the age of 50 she needed a knee replacement of her left knee, which was complicated by a bleeding in the prosthesis, resulting in limited mobility. In 2003, bleeding evaluation in another center revealed a VWF activity of 59\%. In 2005 she needed a cholecystectomy, which was performed with Desmopressin and tranexamic acid pre-operative. No bleeding occurred.

Upon questioning she reported epistaxis at least once a month with a duration of $>5$ minutes, bleeding after minor wounds $>5$ minutes, and heavy menstrual bleeding in the past, requiring iron therapy. Her bleeding score was 14. Family history was negative for bleeding, or bleeding disorders. Physical examination did not reveal any abnormalities, apart from a few bruises on her forearms $(2 \mathrm{~cm})$. Laboratory results showed no abnormalities in complete blood count and coagulation screening tests, apart from a prolonged PFA (CT-EPI 240s, CT-ADP 133s). VWF levels (VWF activity 76\%), light transmission aggregometry (LTA) and ATP release were normal. All coagulation factors, including fibrinolytic proteins were within the normal range. The PFA was repeatedly abnormal in both cartridges. She was diagnoses as a patient with, bleeding of unknown cause (BUC; PFA-only). A treatment plan was provided with tranexamic acid and DDAVP. During surgery for her knee replacement, after the DDAVP gift, the PFA normalized (CT-EPI 102s, CT-ADP 77s), her VWF activity raised to $226 \%$, and FVIII increased to $368 \%$. Thrombosis prophylaxis was started. No bleeding or thrombotic complications occurred.

\section{Definition of a mild bleeding disorder: let's keep it simple!}

Classification of disease severity in bleeding disorders is not straightforward and differs between the different diseases. In hemophilia $A$ and $B$, the severity of the disease is based on the level of factor VIII or IX, with a factor level of $<1 \%$ classifying as severe hemophilia, $1-5 \%$ as moderate and $5-40 \%$ as mild hemophilia ${ }^{1}$. In von Willebrand disease type 1 , there is no uniform classification: a VWF level below $30 \%$ defines VWD type 1 , with a subgroup classified as severe VWD type 1 , when VWF activity is below $10 \%^{2}$. When VWF levels are between $30-50 \%$, definitions vary from mild VWD type 1 to 'possible' VWD type 1 to low VWF, implicating that a VWF level within this range is not a definite disease ${ }^{3,4}$. In the recently published ASH ISTH NHF WFH 2021 guidelines on the diagnosis of von Willebrand disease, a VWF level of 30$50 \%$ indicates VWD type 1 , if it is accompanied by abnormal bleeding 5 .

The platelet function disorders Glanzmann thrombasthenia and Bernard Soulier, are regarded as severe PFD, based on the severe bleeding phenotype associated with 
these disorders. Mild platelet function disorders are classified as such, based on the mild bleeding phenotype according to some, but also on the mild or non-specific laboratory abnormalities of platelet aggregation and secretion according to others ${ }^{6,7}$.

Another complicating factor in the classification of bleeding disorders is, that there is often no clear correlation between the severity of abnormalities found in the laboratory and bleeding phenotype, for example in carriers of hemophilia ${ }^{8}$ and in patients with low VWF or FXI deficiency ${ }^{9}$. The explanation for discrepancy in bleeding phenotypes is likely to be multifactorial. Various genetic or acquired factors might contribute to plasma factor levels and hence influence bleeding in these patients ${ }^{10}$.

In 2016 the European Hematology Association (EHA) established an International Working Group (IWG) to develop a series of guidelines on mild bleeding disorders. In their first communication they acknowledge that a precise definition of a mild bleeding disorder is lacking ${ }^{11}$. They also define the ' $M$ ' in the acronym MBD as Mild to Moderate. However, we question the usefulness of adding another grading step to an already complex definition. In Table 8.1. the proposed definitions for mild/moderate bleedings disorders, mild/moderate bleeding symptoms and mild/moderate bleeding phenotype, are given:

Table 8.1 Recommended Definitions and Terminology for the Description of Inherited/Congenital bleeding disorder, the Severity of Bleeding Symptoms and Associated Bleeding Phenotypes (adapted from Rodeghiero et al.) ${ }^{11}$.

\begin{tabular}{ll}
\hline MBD & Denomination used to collectively describe any bleeding disorder (BD) \\
associated with a mild to moderate bleeding phenotype. The bleeding \\
phenotype is often not distinctively manifest or predictable at the time of \\
diagnosis. It may become evident later in life or only after significant \\
hemostatic challenges. A typical example may be von Willebrand disease. \\
Any BD consistently associated with a mild bleeding phenotype in the great \\
majority of affected, present at the time of diagnosis or reasonably \\
predictable. Examples: mild hemophilia A and B (FVIII or FIX > 5\% and < 40\%); \\
some disorders with a reduced platelet count and/or function defects. \\
Any BD consistently associated with a moderate bleeding phenotype in the \\
great majority of the affected. The bleeding phenotype may be already \\
manifest at the time of diagnosis or become evident later in life or after \\
disorder \\
significant hemostatic challenges. Examples: moderate hemophilia A and B \\
(FVIII or FIX between 1\% and 5\%); some disorders with a reduced platelet \\
count and/or function defects. \\
Easy bleeding consisting in frequent (>5 times/year) bruising, nose, gum, mild \\
menorrhagia or other minor hemorrhagic symptoms that may be disturbing for \\
the patient, although not causing the patient to seek medical care. The single \\
bleeding manifestations would have otherwise received score 0 to 1 with ISTH \\
BAT. \\
Any bleeding event that does not require specific intervention by a doctor \\
beyond simple consultation, referral for investigation or general education on \\
admitted medications and life style (score 1 or 2). Antifibrinolytic and \\
hormonal/local IUD treatment for heavy menstrual bleeding or for post- \\
partum hemorrhage might be required.
\end{tabular}


Table $8.1 \quad$ (continued)

\begin{tabular}{ll}
\hline Moderate bleeding & $\begin{array}{l}\text { Any bleeding event that requires active medical intervention or treatment to } \\
\text { control hemorrhage and does not exceed score 3. Subdural hematoma not } \\
\text { requiring treatment or prolonged observation is considered a moderate }\end{array}$ \\
symptom. & $\begin{array}{l}\text { Excessive bleeding tendency represented by more or less frequent mild } \\
\text { bleeding symptoms that, despite being perceived as alarming by the patient or } \\
\text { patient family members, are not organ-threatening or fatal and do not require } \\
\text { medical intervention (apart from referral for investigation or general education } \\
\text { on medications and life style). They may appear late in life. May be } \\
\text { spontaneous (epistaxis), but are usually provoked by significant hemostatic } \\
\text { challenges such as menstruation, dental extraction, surgery or trauma. The } \\
\text { impact of the perceived QoL should be reported but be minimal and not or } \\
\text { minimally affecting daily life style. Bleeding episodes do not exceed score } 2 \\
\text { ISTH BAT. } \\
\text { Excessive bleeding tendency characterized by mild, but also by one or more } \\
\text { moderate symptoms not exceeding score 3 ISTH BAT. On the overall the } \\
\text { moderate bleeding phenotype is of intermediate severity. Single bleeding } \\
\text { episodes may be spontaneous or secondary to minimal trauma and may } \\
\text { require specific medical intervention. Organ-threatening damage is possible, } \\
\text { but rare and never fatal. In these patients, the frequency of bleeding } \\
\text { symptoms is variable but they may occur at an early age and should call for } \\
\text { prompt investigation. }\end{array}$ \\
\hline
\end{tabular}

ISTH BAT, International Society of Thrombosis and Hemostasis Bleeding Assessment Tool; MBD, mild to moderate bleeding disorder; QoL, quality of life.

The distinction between mild and moderate is quite confusing and seems to be based on factor levels (bleeding disorder), the need for medical intervention or not (bleeding symptom), the effect on quality of life (bleeding phenotype) and on the grading of bleeding episodes on the ISTH BAT score (bleeding symptom and bleeding phenotype). However, in daily practice a patient does not present with a clear cut bleeding history and a patient with mild bleeding symptoms can have a moderate bleeding phenotype resulting from a mild bleeding disorder. The IWG also acknowledges that the discrimination of abnormal bleeding and the assessment of severity of bleeding symptoms and bleeding phenotypes is dependent on the subjective judgement of an expert clinician, integrating many aspects such as age, comorbidities, number of hemostatic challenges, recall bias and affected family members ${ }^{11}$.

To keep things simple, in our opinion, bleeding disorders without severe phenotype or classifying as severe or moderate based on factor level, should be defined as mild bleeding disorders.

\section{Diagnosing mild bleeding disorders: clinical utility is in the eyes of the beholder}

In Chapter 5, we performed a comparison of effectiveness, hospital resource use and costs, and patient burden of two diagnostic approaches for patients referred for 
bleeding evaluation. The all-in-one diagnostic approach had a higher diagnostic yield, and provided a faster diagnosis with less patient burden at a higher cost, compared to the standard stepwise approach. These findings open the discussion to what the clinical utility of the search and final diagnosis of a mild bleeding disorder is.

The clinical utility of a test or diagnostic work-up refers to a test outcome that provides valuable information to the person being tested and the clinician treating this person ${ }^{12}$. The information can be used to apply effective treatment or preventive therapy. If no interventions are available, or if there is no final diagnosis, there may also be benefits associated with this knowledge ${ }^{13}$.

Patients with mild bleeding disorders have no increased mortality. However, they may suffer from increased morbidity, like uncontrolled bleeding after surgery, trauma or childbirth ${ }^{14-16}$. Also, bleeding symptoms can be perceived as alarming by the patient or family members and can have an impact on quality of life $(\mathrm{Q} o L)^{11}$. A large study in the Netherlands in more than 500 adult patients with VWD (VWF levels 10-30\%) reported a lower health related quality of life for general health, vitality (energy and fatigue) and for physical component summary compared to the general population ${ }^{17,18}$. Another study, including patients VWF levels of $30-50 \%$, also found significant reductions in quality of life for general health, vitality and social functioning ${ }^{19}$. Especially, heavy menstrual bleeding in female patients with mild VWD reduced quality of life ${ }^{20,21}$. The impact of VWD on health related quality of life already started at a young age ${ }^{22}$ and the risk of bleeding, accompanied by lower QoL, remained constant throughout life ${ }^{9}$. In 2020 another Dutch study investigated the quality of life of 165 patients with congenital platelet function disorders. They reported decreased physical functioning, limitations in daily activities due to physical health problems, limitations in social activities, decreased energy levels, more fatigue and pain, and lower general health status in this population compared to the general Dutch population ${ }^{23}$.

After correct diagnosis of a MBD, preventive treatment e.g. during menstrual cycle or peri-operatively can be applied, in order to prevent heavy bleeding. Other positive patient-related outcomes of extensive investigations for a MBD have been described: reassurance of complete investigation even if no diagnosis is found; explanation for alarming bleeding symptoms; tailored information and education regarding lifestyle; avoidance of certain medication; consideration of job-related risks; women's issues; preparation for dental or surgical procedures; targeted investigation of family members and genetic counseling ${ }^{11,24}$. However, also negative outcomes have been considered, like unnecessary concerns and exaggerated perception of bleeding risk; improper delay of dental or surgical procedures and unwarranted treatment, possible causing a risk of thrombosis ${ }^{11,15}$. 
If a patient seeks consultation for bleeding symptoms, this indicates that the perceived complaints are of concern, worry or anxiety to the patient. Before initiating any investigation, the pros and cons of a thorough bleeding investigation as depicted above, need to be discussed with the patient. Specific attention has to be paid to the clinical utility of the investigation for the individual patient or situation, and to management of the patient's expectations. Shared-decision making between a wellinformed patient and an experienced clinician can then guide further investigation.

The bleeding assessment tool is of limited additional diagnostic value to the work-up of patients referred for bleeding evaluation in daily practice

Assessment of bleeding requires recording of bleeding sites, the amount of blood lost, the frequency and duration of bleeding episodes, easy bruising in unusual sites, bleeding proportionate to the magnitude of injury, and the need for blood transfusion, iron deficiency therapy or surgery to stop bleeding ${ }^{25}$. A bleeding assessment tool (BAT) can summarize a great deal of this clinical information.

In Chapter $\mathbf{2}$ we performed a systematic review of diagnostic studies assessing the performance of the BAT in patients referred to a secondary care setting for expert evaluation of bleeding symptoms. Our results showed that a negative BAT cannot be used to rule out mild bleeding disorders, and a positive BAT increases the likelihood of having a mild bleeding disorder, so further investigation is warranted. The main advantage of the BAT is that it offers a complete and structured interview, addressing bleeding symptoms in different organs, especially in settings were standardization of clinical assessment is mandatory. However, the BAT is of limited additional diagnostic value to the work-up of patients referred for bleeding evaluation in clinical practice ${ }^{26}$. Literature published after the final search date of our review in June 2017, shows that the discussion about the diagnostic value of the BAT is still ongoing. Some authors suggest that the ISTH (self) BAT is useful as a screening tool in patients with a suspected $\mathrm{PFD}^{27,28}$, or that it is useful for identifying subjects requiring laboratory evaluation for a suspected PFD once VWD is excluded ${ }^{29}$. Other authors found low positive and negative predictive values of the Vicenza based BATs and the ISTH BAT for the diagnosis of a bleeding disorder in patients referred for bleeding evaluation ${ }^{30}$.

As discussed in Chapter $\mathbf{2}$ and as our first patient case shows, care should be taken when interpreting the BAT score in young people or in subjects with no or few previous hemostatic challenges ${ }^{11}$. Moreover, within the BAT scoring system the family history is not taken into account. It is also important to keep in mind that any prophylactic treatment can falsely increase the overall bleeding score. Furthermore, clinical situations as an appropriate explanation for bleeding at the time of surgery or 
post-partum (e.g. uterine atony) should be considered. Another issue is that, in the BAT score, the frequency of bleeding is not accounted for, only the most severe bleeding episode is scored. Thus the score becomes saturated after the first severe event, and does not take into account the frequency of bleeding events ${ }^{31}$. Therefore, the IWG proposes a new category of nuisance bleeding, to account for patients suffering from frequent and disturbing but mild bleeding symptoms (Table 8.1). They advise that patients with nuisance bleeding, independent of the BAT score, deserve extensive laboratory investigation. Also, guidance is given to specific situations in which further investigation is appropriate, e.g. patients with any grade 1 bleeding at very young age, or with a history of infancy-specific bleeding symptoms (e.g. umbilical stump bleeding $)^{11}$.

It has also been shown that the results of a quantitative bleeding score highly correlate with the estimation of the physician who interviewed the patients $(R=0.75$, $p<0.0001)^{14}$. We therefore conclude that a personal, structured and brief but complete interview, guided by, but not limited to, a bleeding assessment tool can support the clinical judgement of an experienced physician in identifying patients with abnormal bleeding.

\section{Using the currently available laboratory tests, we will never know why most patients bleed ${ }^{4}$}

Primary hemostasis depends on many factors involved in the interaction of VWF and platelets at the site of vessel wall injury. Quantitative or qualitative defects in any of these factors, known or unknown, and disturbances in the complex interaction of other blood components might cause or contribute to bleeding 4,32 . Given this complexity, it is not surprising that standard laboratory testing only identifies a hemostatic defect in $25-50 \%$ of patients referred for bleeding evaluation ${ }^{10,33,34}$.

Patients with a significant bleeding history and no reproducible abnormality with the standard tests of hemostasis are classified as patients with bleeding of unknown cause $(B \cup C)^{35}$. These patients suffer from the same bleeding as patients with a diagnosed mild bleeding disorder ${ }^{10,34,36,37}$ and are often provided with a treatment plan to prevent bleeding after surgery or childbirth ${ }^{38}$. Bleeding without a diagnosis is likely to cause a psychological strain on affected individuals and physicians for a few reasons: lack of diagnosis or explanation for bleeding gives the uncertainty that 'something is missed'; counselling families about inheritance and implications for pregnancy is difficult; a treatment plan according to existing guidelines cannot be established and is, as our second case shows, not without (thrombotic) risks ${ }^{33,34}$.

It is expected that patients with BUC suffer from a heterogeneous group of disorder(s) of high frequency and unknown pathogenesis ${ }^{10}$. In this population new 
techniques will hopefully identify new disease entities. Many new hemostatic tests like thrombin generation, fibrinolysis tests and viscoelastic testing, but also genetic testing have been performed in this patient group. Results are interesting, but until now, no clear clues have been found to why these patients bleed ${ }^{34,38-42}$. In one of the first reports identifying patients with BUC, 19\% of the patients had a prolonged bleeding time ${ }^{10}$. Although this test is obsolete due to the invasive character and poorly standardization of the test, it was the only test so far measuring coagulation in vivo ${ }^{43}$. Succeeding the bleeding time, the platelet function analyzer (PFA) is also strongly criticized $\mathrm{on}^{44}$. However, the PFA is the only standard coagulation test incorporating blood flow into its model.

In Chapter 3 we investigated the diagnostic performance of the PFA-200 and Multiplate for platelet function disorders as detected by light transmission aggregometry in different patient populations. We found that PFA and Multiplate were not able to discriminate between preoperative and referred patients with and without mild platelet function disorders, and therefore are not useful as screening tests to rule out mild PFD in these populations. Both PFA and Multiplate were able to detect severe platelet function disorders ${ }^{45}$. In Chapter 4 we investigated patients with bleeding of unknown cause with a prolonged PFA as the only aberrant finding in the work-up of bleeding evaluation. With use of a multiparameter microfluidic test, whole-blood thrombus formation under shear was investigated, taking into account the blood flow component and cellular interactions. We found that microfluidic test parameters were impaired in PFA-only patients compared to healthy controls. Abnormalities reached to levels seen in patients with established bleeding disorders, such as Glanzmann thrombasthenia and VWD. The microfluidic test sensed single or combined effects of low-normal VWF and low-normal platelet adhesion, activation and aggregation in these patients, suggesting a shear-dependent platelet function defect, not detected by the static conventional hemostatic tests.

So why is it that, with all available tests, we often do not know why patients bleed? Bleeding is likely to be influenced by the interaction and accumulation of small defects or polymorphisms in different genes or acquired factors. Increased variability in penetrance and expressivity can cause large inter-individual variability in synthesis of a functional factor ${ }^{39}$ and the combination of one of more coagulation factors in there lower range can trigger bleeding ${ }^{46}$. This partially explains why bleeding in patients with bleeding of unknown cause may be controlled with empirically use of therapeutic agents like desmopressin and tranexamic acid ${ }^{14}$, but also why the addition of an acquired factor (aspirin) elicits bleeding in some of these patients ${ }^{10,25}$. Another important consideration is that the function of the vessel wall is not evaluated by standard available coagulation tests. There are multiple diseases causing bleeding 
symptoms not related to a defect in platelets or coagulation factors (e.g. EhlersDanlos symptoms, vasculitis, Cushing syndrome) in which a dysfunction of endothelial cells or subendothelial integrity may account for the bleeding ${ }^{33}$.

\section{Bleeding risk in patients with mild bleeding disorders: putting together pieces of evidence}

As described above in patients with BUC, bleeding phenotypes in patients with mild bleeding disorders can be variable because of interplay between genetic and environmental factors. Also, the frequency of combined hemostatic defects in bleeding patients is higher than is predicted by chance ${ }^{10}$. The occurrence of severe bleeding, or a high BAT score in a patient diagnosed with a mild bleeding disorder, should therefore always raise the consideration of an additional hemostatic defect that may tip the hemostatic balance over towards bleeding ${ }^{46}$. In line with that, a single laboratory measurement and even identification of a specific molecular defect cannot be used as a marker of individual bleeding risk ${ }^{24}$. Bleeding in MBD might be comparable to myocardial infarction or venous thromboembolism (VTE) ${ }^{4}$, which often occur in subjects with several individually weak risk factors that cooperate to cause an event ${ }^{47}$. The use of data-derived risk management for cardiovascular disease and VTE is daily practice and this might also be applicable to bleeding.

An interesting approach has been the Bayesian analysis of bleeding symptoms, VWF level and VWF inheritance to predict the likelihood of VWD type 1 among subjects enrolled in the European VWD type 1 study ${ }^{48}$. According to the Bayes theorem, the baseline probability of a disease (prevalence) is multiplied by the likelihood ratio (LR) given by a clinical or laboratory finding to obtain a posterior probability of the disease in the studied subject. For example, in this study, the final odds of having VWD type 1 (prevalence of $0.1 \%$ ) ranged from 0.9 in a patient with one affected family member, a bleeding score of 3 and a VWF level of $20-40 \%$ to $>10.000$ in a patient with three affected family members, a bleeding score of 5 and a VWF level of $<20 \%$. This method could be extended to all patients referred for evaluation of bleeding symptoms, but unfortunately, this is hampered by the lack of data from large multicenter studies on cohorts of MBD, other than VWD, and the unclear genetic model, to compute the LR.

Alternatively, when discussing patients referred for bleeding disorders in a multidisciplinary team, presenting all the different 'risk factors' could provide the basis for a balanced approach and judgement of clinical and laboratory data in order to decide on the likelihood of a bleeding disorder being present (or on the level of certainty that it is absent). This discussion should at least include the pretest probability of a bleeding disorder based on the incidence of a bleeding disorder in the 
population tested (e.g. in patients referred for bleeding evaluation to the hematologist up to $44 \%{ }^{14,49}$ ), the reason for referral, a critically appraised history and BAT score, the family history, sport activities, the results of repeated laboratory testing, genetic findings, possibilities for vascular disease and bleeding on aspirin or NSAIDs/natural supplements. Apart from these, referral for specific situations like high risk surgery or pregnancy and labor should guide clinical practice (Table 8.2). The multidisciplinary assessments and careful judgement of the pieces of evidence for or against a bleeding disorder should provide maximal information about the bleeding risk in each individual patient and circumstance.

\section{Future perspectives (part I)}

By the end of this thesis, the bleeding algorithm Maastricht (BAM) is established. Based on the results of this thesis, the work-up of patients referred for bleeding evaluation has changed from a stepwise approach to an all-in-one approach.

In all patients referred for bleeding evaluation a critical appraisal of (nuisance) bleeding symptoms guided by a bleeding assessment tool is performed in the context of clinical information on: reason for referral, age; sex; personal history of hemostatic challenges; medication/supplement use; family history; possibilities for vascular disease, and bleeding on aspirin or NSAIDs. Then, all routinely available hemostatic tests are performed. Finally, DNA analysis or WES can be performed in specific cases. All this information and the test results will be discussed in a multidisciplinary team, putting together the pieces of evidence and resulting in carefully judged indication of the likelihood of having a bleeding disorder. Implications for treatment will be established, and a personalized treatment plan is formed, taking into account specific situations like high risk surgery or pregnancy.

Further research on experimental hemostatic assays in bleeding patients (e.g. multiparameter microfluidic tests, ROTEM based platelet and fibrinolytic assays, or thrombin generation) including critical appraisal of their diagnostic value, will bring these tests closer to clinical practice and may fill some of the existing knowledge gaps. Ultimately, this will lead to better guidance and treatment of patients with mild bleeding disorders including patients with bleeding of unknown cause, during their lives and in all possible situations. 


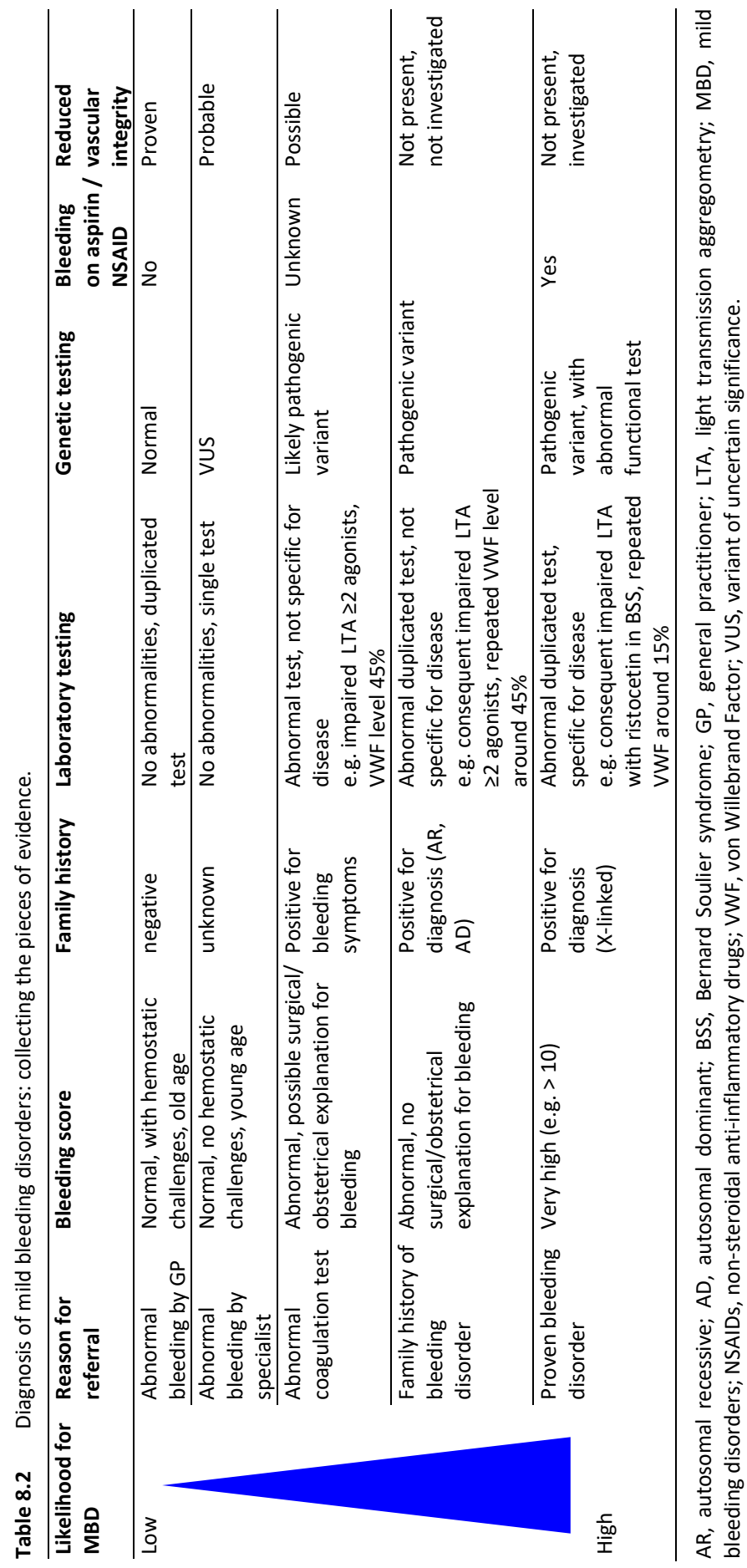




\section{Part II Mechanisms of bleeding in chemotherapy induced thrombocytopenia}

\section{Balance of hemostasis in hemato-oncology patients: an extra dimension}

In hemato-oncology patients, chemotherapy can cause thrombocytopenia in different ways: by reducing pluripotent stem cells or subsequent megakaryocyte progenitors (alkylators, cyclophosphamide) or by inducing platelet apoptosis (cisplatin) ${ }^{50}$. The primary goal of thrombocytopenic management following chemotherapy is the prevention of major bleeding with minimal use of platelet transfusions.

In Chapter 6 we focused on thrombocyte dysfunction in patients with chemotherapy induced thrombocytopenia (CIT) and found that mitochondrial function was markedly reduced in platelets, as evidenced by a low mitochondrial membrane potential and low oxygen consumption, while the mitochondrial content was normal. Moreover, the mitochondrial impairments coincided with elevated levels of reactive oxygen species. This platelet dysfunction only appeared after two days of chemotherapy, suggesting origination in the megakaryocytes. In patients with bone marrow recovery, platelet function improved. Apart from lower platelet count, platelet dysfunction might contribute to bleeding in these patients ${ }^{51}$. Other studies investigating platelet dysfunction performed flowcytometry and also found that agonist induced platelet reactivity was inversely correlated with bleeding in CIT patients ${ }^{52}$, and reduced platelet aggregation and activation predicted bleeding better than platelet count alone ${ }^{53}$. These and our results indicate that the use of thrombopoietin receptor agonists, although a positive effect on platelet nadir and duration of thrombocytopenia has been described ${ }^{54}$, might be of limited use for the prevention of bleeding in these patients.

In Chapter 7, fibrinolysis in CIT patients was evaluated with tPA-ROTEM. Results showed that clots of CIT patients were more susceptible to fibrinolysis and a subgroup of patients had a hyperfibrinolytic profile. Platelet transfusions resulted in less hyperfibrinolytic profiles in many, but not all CIT patients ${ }^{55}$. To prevent bleeding in patients with a hyperfibrinolytic profile, instead of platelet transfusion, this selected group of patients might be treated with tranexamic acid (TXA). Tranexamic acid is a lysine analogue and inhibits conversion of plasminogen to plasmin to slow down clot lysis. Results of a recent Dutch nationwide survey showed that tranexamic acid in CIT patients is predominantly prescribed as supportive care in case of a bleeding tendency or a clinically relevant bleeding in addition to platelet transfusion ${ }^{56}$.

Literature about the use of antifibrinolytics for the prevention of bleeding in CIT patients is conflicting. A Cochrane systematic review and meta-analysis published in 2016 concluded that there is uncertainty about the reduction in bleeding risk by use 
of antifibrinolytics in hemato-oncology patients, due to the small number of study participants and low quality of evidence of the studies performed ${ }^{57}$. A subsequent meta-analysis published in 2017 evaluated the safety and efficacy of lysine analogues in a total of 1177 cancer patients. The use of lysine analogues significantly decreased blood loss and transfusion risk, without increasing the risk of venous thromboembolism ${ }^{58}$. However, only 2 studies with hemato-oncology patients were included, the same as in the Cochrane review published earlier.

Three interesting trials were recruiting participants in 2016 and were not included in this Cochrane review (PROBLEMA 2014 (still recruiting), TREATT 2015 (still recruiting), A-TREAT 2015). The results of the A-TREAT trial were presented at the ASH in 2020 and published in abstract form ${ }^{59}$. This randomized controlled trail assessed prophylactic tranexamic acid administration in addition to routine platelet transfusion. The study included 165 patients in each study arm and found no difference in the rate of WHO grade $\geq 2$ bleeding, and platelet and blood cell transfusion rates between both study arms. In the TXA arm, there was an increased rate of central line occlusions. This preliminary publication suggests that TXA should not be routinely used for preventing bleeding in addition to prophylactic platelet transfusions.

Another factor contributing to bleeding risk in these patients is reduced vascular integrity. A study investigating markers of endothelial insufficiency and inflammation in CIT patients found an increase in grade 2 bleeding in patient with higher urine albumin/creatinine ratio and an increase in the odds of grade 2 bleeding for every doubling of serum C-reactive protein as markers for endothelial damage ${ }^{60}$. Furthermore, lack of thrombocytes leads to ultrastructural changes and increased permeability of the endothelium ${ }^{61}$.

The greatest difficulty in preventing bleeding in CIT patients is that cancer patients are at risk of thrombosis, and thrombocytopenia is not protective. In a large cohort of more than 1500 patients undergoing hematopoietic stem cell transplantation, $4.6 \%$ of the patients developed venous thromboembolism, $34 \%$ of these patients had platelet count less than $50 \times 10^{9} / \mathrm{L}$ and $13 \%$ occurred at a platelet count of less than $20 \times 10^{9} / \mathrm{L}$. Clinically significant bleeding occurred in $15.2 \%$ of the patients, $3.6 \%$ had a fatal bleeding. Bleeding was associated with anticoagulant therapy ${ }^{62}$. The balance of bleeding and thrombotic risk in hemato-oncology patients is therefore as challenging as, or even more complex than it is, in patients with 'benign' bleeding disorders. 


\section{Future perspectives (part II)}

Our next focus in CIT patients will be on the role of leukocytes in coagulation. Leukocytes, especially monocytes and neutrophils, influence hemostasis in several different ways and lack of leukocytes in CIT patients might influence their hemostatic potential. Normally, monocytes express protein $C$ receptor, thrombomodulin and tissue factor pathway inhibitor, thereby inhibiting coagulation. However, upon activation by inflammatory factors, monocytes express tissue factor (TF) and it has been suggested that monocytes are the main source of blood-borne $\mathrm{TF}^{63,64}$. Furthermore, monocytes and macrophages are able to secrete microparticles coated with $\mathrm{TF}^{65}$. Also, activated neutrophils are able to form neutrophil extracellular traps (NETs). NETs consists of fibers made by histones and DNA released by these cells in order to trap pathogens within blood. In coagulation, NETs provide a negatively charged surface where contact activation occurs. The structure also contribute to formation of thicker clot fibers with greater mechanical stability ${ }^{63}$.

We will also investigate the use of ROTEM in plasma, to gain more insight in the specific effects of plasma components on coagulation and to be able to measure stored samples, or samples from other centers or studies (e.g. the Dutch Bleeding In Thrombocytopenia Explained (BITE) study ${ }^{66}$ ). Finally, we will investigate native rotational thromboelastometry (NATEM) ROTEM in different patient populations. In this assay the patient sample is solely re-calcified in absence of an activator of hemostasis in order to test the endogenous hemostatic potential. 


\section{References}

1. Srivastava A, et al. WFH Guidelines for the Management of Hemophilia, 3rd edition. Haemophilia 2020;26 Suppl 6:1-158.

2. Federici AB. Clinical diagnosis of von Willebrand disease. Haemophilia 2004;10(s4):169-76.

3. Leebeek FW, Eikenboom JC. Von Willebrand's Disease. N Engl J Med 2016;375(21):2067-80.

4. Sadler JE. Low von Willebrand factor: sometimes a risk factor and sometimes a disease. Hematology Am Soc Hematol Educ Program 2009:106-12.

5. James PD, et al. ASH ISTH NHF WFH 2021 guidelines on the diagnosis of von Willebrand disease. Blood Adv 2021;5(1):280-300.

6. Norman JE, et al. How should we test for nonsevere heritable platelet function disorders? Int J Lab Hematol 2014;36(3):326-33.

7. Quiroga T, et al. Diagnosis of mild platelet function disorders. Reliability and usefulness of light transmission platelet aggregation and serotonin secretion assays. British Journal of Haematology 2009;147(5):729-36.

8. James PD, et al. Evaluation of the utility of the ISTH BAT in haemophilia carriers: a multinational study. Haemophilia 2016;22(6):912-8.

9. Tosetto A, et al. A quantitative analysis of bleeding symptoms in type 1 von Willebrand disease: results from a multicenter European study (MCMDM-1 VWD). J Thromb Haemost 2006;4(4):766-73.

10. Quiroga T, et al. High prevalence of bleeders of unknown cause among patients with inherited mucocutaneous bleeding. A prospective study of 280 patients and 299 controls. Haematologica 2007; 92(3):357-65.

11. Rodeghiero F, et al. Fundamentals for a Systematic Approach to Mild and Moderate Inherited Bleeding Disorders: An EHA Consensus Report. Hemasphere 2019;3(4):e286.

12. Bossuyt PM, et al. Beyond diagnostic accuracy: the clinical utility of diagnostic tests. Clin Chem 2012; 58(12):1636-43.

13. Lesko L, Zineh I, Huang SM. What Is Clinical Utility and Why Should We Care? Clin Pharmacol Ther 2010;88:729-33.

14. Quiroga T, Mezzano D. Is my patient a bleeder? A diagnostic framework for mild bleeding disorders. Hematology Am Soc Hematol Educ Program 2012;2012:466-74.

15. Greaves M, Watson HG. Approach to the diagnosis and management of mild bleeding disorders. J Thromb Haemost 2007;5 Suppl 1:167-74.

16. Orsini $\mathrm{S}$, et al. Bleeding risk of surgery and its prevention in patients with inherited platelet disorders. Haematologica 2017;102(7):1192-203.

17. Sanders YV, et al. [Von Willebrand disease in the Netherlands: the WiN study]. Ned Tijdschr Geneeskd 2014;158:A6518.

18. de Wee EM, et al. Health-related quality of life among adult patients with moderate and severe von Willebrand disease. J Thromb Haemost 2010;8(7):1492-9.

19. Xu Y, et al. Relative contributions of bleeding scores and iron status on health-related quality of life in von Willebrand disease: a cross-sectional study. Haemophilia 2017;23(1):115-21.

20. Govorov I, et al. Heavy menstrual bleeding and health-associated quality of life in women with von Willebrand's disease. Exp Ther Med 2016;11(5):1923-9.

21. Rae $\mathrm{C}$, et al. Bleeding disorders, menorrhagia and iron deficiency: impacts on health-related quality of life. Haemophilia 2013;19(3):385-91.

22. De Wee EM, et al. Impact of von Willebrand disease on health-related quality of life in a pediatric population. J Thromb Haemost 2011;9(3):502-9.

23. Blaauwgeers MW, et al. Congenital platelet disorders and health status-related quality of life. Res Pract Thromb Haemost 2020;4(1):100-5.

24. Rodeghiero F, Tosetto A, Castaman G. How to estimate bleeding risk in mild bleeding disorders. J Thromb Haemost 2007;5:157-66.

25. Mezzano D, Quiroga T. Diagnostic challenges of inherited mild bleeding disorders: a bait for poorly explored clinical and basic research. J Thromb Haemost 2019;17(2):257-70. 
26. Moenen $F$, et al. The diagnostic accuracy of bleeding assessment tools for the identification of patients with mild bleeding disorders: A systematic review. Haemophilia 2018;24(4):525-35.

27. Adler $\mathrm{M}$, et al. Diagnostic utility of the ISTH bleeding assessment tool in patients with suspected platelet function disorders. J Thromb Haemost 2019;17(7):1104-12.

28. Punt MC, et al. Reliability and Feasibility of the Self-Administered ISTH-Bleeding Assessment Tool. TH Open 2019;3(4):e350-5.

29. Gresele P, et al. Validation of the ISTH/SSC bleeding assessment tool for inherited platelet disorders: A communication from the Platelet Physiology SSC. J Thromb Haemost 2020;18(3):732-9.

30. Gebhart J, et al. The discriminatory power of bleeding assessment tools in adult patients with a mild to moderate bleeding tendency. Eur J Intern Med 2020;78:34-40.

31. Bowman ML, James PD. Bleeding Scores for the Diagnosis of von Willebrand Disease. Semin Thromb Hemost 2017;43(5):530-9.

32. Sang Y, et al. Interplay between platelets and coagulation. Blood Rev 2021;46:100733.

33. Thomas W, Downes K, Desborough MJR. Bleeding of unknown cause and unclassified bleeding disorders; diagnosis, pathophysiology and management. Haemophilia 2020.

34. Gebhart J, et al. High proportion of patients with bleeding of unknown cause in persons with a mildto-moderate bleeding tendency: Results from the Vienna Bleeding Biobank (VIBB). Haemophilia 2018; 24(3):405-13.

35. Zegers SAM, et al. Diagnostic work up of patients with increased bleeding tendency. Haemophilia 2020;26(2):269-77.

36. Sramek A, et al. Usefulness of Patient Interview in Bleeding Disorders. Arch Intern Med 1995;155(13):1409-15.

37. Relke $\mathrm{N}$, et al. The bleeding score: Useful in predicting spontaneous bleeding events in adults with bleeding of unknown cause? Haemophilia 2020;26(2):e31-3.

38. MacDonald S, et al. Characterization of a large cohort of patients with unclassified bleeding disorder; clinical features, management of haemostatic challenges and use of global haemostatic assessment with proposed recommendations for diagnosis and treatment. Int J Lab Hematol 2019;42(2):116-25.

39 Downes K, et al. Diagnostic high-throughput sequencing of 2396 patients with bleeding, thrombotic, and platelet disorders. Blood 2019;134(23):2082-91.

40. Hofer $\mathrm{S}$, et al. Thrombin-generating potential, plasma clot formation, and clot lysis are impaired in patients with bleeding of unknown cause. J Thromb Haemost 2019;17(9):1478-88.

41. Veen CSB, et al. Evaluation of thromboelastometry, thrombin generation and plasma clot lysis time in patients with bleeding of unknown cause: A prospective cohort study. Haemophilia 2020;26(3): e106-15.

42. MacDonald S, et al. Investigation of patients with unclassified bleeding disorder and abnormal thrombin generation for physiological coagulation inhibitors reveals multiple abnormalities and a subset of patients with increased tissue factor pathway inhibitor activity. Int J Lab Hematol 2020; 42(3):246-55.

43. Paniccia R, et al. Platelet function tests: a comparative review. Vasc Health Risk Manag 2015;11: 133-48.

44. Hayward C, et al. Platelet function analyzer (PFA)-100 closure time in the evaluation of platelet disorders and platelet function. J Thromb Haemost 2006;4(2):312-9.

45. Moenen FCJ, et al. Screening for platelet function disorders with Multiplate and platelet function analyzer. Platelets 2019;30(1):81-7.

46. Chauleur $\mathrm{C}$, et al. Some hemostasis variables at the end of the population distributions are risk factors for severe postpartum hemorrhages. J Thromb Haemost 2008;6(12):2067-74.

47. Brouwer JL, et al. The pathogenesis of venous thromboembolism: evidence for multiple interrelated causes. Ann Intern Med 2006;145(11):807-15.

48. Tosetto A, Castaman G, Rodeghiero F. Evidence-based diagnosis of type 1 von Willebrand disease: a Bayes theorem approach. Blood 2008;111(8):3998-4003.

49. Tosetto A, et al. Prospective evaluation of the clinical utility of quantitative bleeding severity assessment in patients referred for hemostatic evaluation. J Thromb Haemost 2011;9(6):1143-8. 
50. Leader A, Hofstetter L, Spectre G. Challenges and Advances in Managing Thrombocytopenic Cancer Patients. J Clin Med 2021;10(6).

51. Baaten CCFMJ, et al. Impaired mitochondrial activity explains platelet dysfunction in thrombocytopenic cancer patients undergoing chemotherapy. Haematologica 2018;103(9):1557-67.

52. Batman B, et al. Agonist-induced platelet reactivity correlates with bleeding in haemato-oncological patients. Vox Sanguinis 2017;112(8):773-9.

53. Vinholt PJ, et al. Prediction of bleeding and prophylactic platelet transfusions in cancer patients with thrombocytopenia. Platelets 2016;27(6):547-54.

54. Vadhan-Raj S, et al. Randomized, Double-Blind, Placebo-Controlled, Dose and Schedule-Finding Study of AMG 531 In Chemotherapy-Induced Thrombocytopenia (CIT): Results of a Phase I/II Study. Blood 2010;116(21):1544.

55. Heubel-Moenen $\mathrm{F}$, et al. Fibrinolysis in patients with chemotherapy-induced thrombocytopenia and the effect of platelet transfusion. J Thromb Haemost 2019;17(7):1073-84.

56. Cornelissen LL, et al. Platelet transfusion and tranexamic acid to prevent bleeding in outpatients with a hematological disease: A Dutch nationwide survey. Eur J Haematol 2021;106(3):362-70.

57. Estcourt $\amalg$, et al. Antifibrinolytics (lysine analogues) for the prevention of bleeding in people with haematological disorders. Cochrane Database Syst Rev 2016;3:CD009733.

58. Montroy J, et al. The Safety and Efficacy of Lysine Analogues in Cancer Patients: A Systematic Review and Meta-Analysis. Transfus Med Rev 2017;31(3):141-8.

59. Gernsheimer TB, et al. Effects of Tranexamic Acid Prophylaxis on Bleeding Outcomes in Hematologic Malignancy: The a-TREAT Trial. Blood 2020;136(Supplement 1):1-2.

60. Ypma PF, et al. The association between haemorrhage and markers of endothelial insufficiency and inflammation in patients with hypoproliferative thrombocytopenia: a cohort study. $\mathrm{Br} \mathrm{J}$ Haematol 2020;189(1):171-81.

61. Kitchens CS, Weiss L. Ultrastructural changes of endothelium associated with thrombocytopenia. Blood 1975;46(4):567-78.

62. Gerber DE, et al. The incidence of and risk factors for venous thromboembolism (VTE) and bleeding among 1514 patients undergoing hematopoietic stem cell transplantation: implications for VTE prevention. Blood 2008;112(3):504-10.

63. Swystun LL, Liaw PC. The role of leukocytes in thrombosis. Blood 2016;128(6):753-62.

64. Giesen PL, et al. Blood-borne tissue factor: another view of thrombosis. Proc Natl Acad Sci U S A 1999;96(5):2311-5.

65. Falati $S$, et al. Accumulation of tissue factor into developing thrombi in vivo is dependent upon microparticle P-selectin glycoprotein ligand 1 and platelet P-selectin. J Exp Med 2003;197(11): 1585-98.

66. Cornelissen LL, et al. Risk factors for bleeding in haemato-oncology patients-a nested case-control study: The BITE study protocol (Bleeding In Thrombocytopenia Explained). BMJ Open 2020;10(6): e034710. 


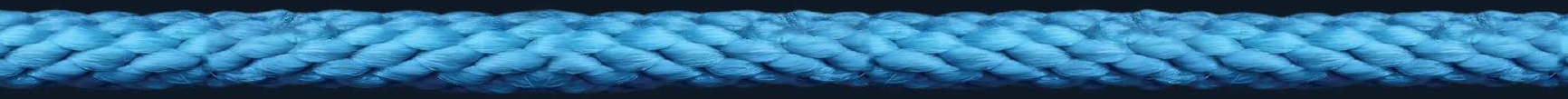


ADDENDUM

Nederlandse samenvatting 



\section{Nederlandse samenvatting}

Milde bloedingsziekten (MBD) omvatten onder andere de ziekte van Von Willebrand (VWD), bloedplaatjesfunctiestoornissen (PFD), milde tot matige stollingsfactor deficiënties en afwijkingen in de fibrinolyse. Correcte identificatie en tijdige herkenning van een milde bloedingsziekte is van belang om bloedingen, met name tijdens of na ingrepen of bevallingen, te voorkomen door behandeling of preventieve therapie in te stellen. Bij patiënten met een milde bloedingsziekte zijn de klinische symptomen heterogeen en moeilijk te onderscheiden van normaal. Ook de interpretatie van laboratorium uitslagen is bij deze patiënten niet altijd eenduidig. Daarnaast wordt in ongeveer $50-75 \%$ van de patiënten die verwezen worden naar de hematoloog voor analyse bloedingsneiging, ondanks uitvoerig laboratoriumonderzoek, geen diagnose gevonden.

Bij patiënten met een hematologische maligniteit leidt de ziekte of behandeling vaak tot een laag aantal bloedplaatjes (trombocytopenie). Dit lage aantal bloedplaatjes verhoogt het risico op bloedingen. Daarom krijgen deze patiënten bij een bloedplaatjes aantal onder de $10 \times 10^{9} / \mathrm{L}$, een profylactische bloedplaatjes transfusie. Echter, sommige patiënten krijgen ondanks deze behandeling een bloeding, terwijl andere patiënten niet gaan bloeden ook al krijgen ze deze behandeling niet.

Dit proefschrift heeft zich gericht op de waarde van verschillende diagnostische testen om patiënten met milde bloedingsziekten te identificeren (deel I) en op het verhelderen van mechanismen van bloeding bij patiënten met door chemotherapie geïnduceerde trombocytopenie (deel II).

In Hoofdstuk 2 werd een systematische review uitgevoerd van negen studies die de diagnostische waarde van een bloedingsvragenlijst en score, de zogenaamde 'bleeding assessment tool' (BAT) onderzochten, bij patiënten die verwezen werden naar een tweedelijns zorginstelling voor evaluatie van bloedingssymptomen. De resultaten van deze review toonden aan dat een negatieve BAT score niet in staat is om milde bloedingsziekten uit te sluiten. Bij een positieve BAT score is er sprake van een verhoogde kans op een milde bloedingsziekte, waarbij uitgebreid laboratoriumonderzoek gerechtvaardigd is. De vraag is echter of dit niet altijd al het geval is, als een patiënt met bloedingssymptomen naar een hematoloog verwezen wordt. De conclusie van dit onderzoek was dat de BAT zorgt voor een complete en gestructureerde anamnese van bloedingssymptomen. Als diagnosticum voor milde bloedingsziekten heeft de BAT echter een beperkte aanvullende diagnostische waarde in de klinische praktijk. 
In Hoofdstuk 3 werd de diagnostische waarde van de bloedplaatjesfunctie testen Multiplate en platelet function analyzer (PFA) geëvalueerd, als screeningstest voor plaatjesfunctiestoornissen in verschillende patiënt populaties. De resultaten toonden aan dat bij preoperatieve patiënten en patiënten die verwezen worden voor evaluatie van bloedingssymptomen de Multiplate en PFA niet in staat zijn om onderscheid te maken tussen patiënten met en zonder milde plaatjesfunctiestoornissen. Ze kunnen dus niet gebruikt worden als screeningstest om milde plaatjesfunctiestoornissen in deze populaties uit te sluiten. Zowel Multiplate als PFA zijn in staat om ernstige plaatjesfunctiestoornissen te detecteren in patiënten die al bekend zijn met deze diagnose.

Hoofdstuk 4 beschrijft flowkamerproeven die werden uitgevoerd bij patiënten met een verlengde PFA als enige afwijkende bevinding in hun diagnostisch onderzoek naar bloedingsneiging. De flowkamerproeven lieten zien dat er bij deze patiënten sprake is van minder bloedplaatjes adhesie, aggregatie en activatie en verminderde stolselvorming in vergelijking met gezonde controles. De conclusie was dan ook dat er in deze patiënten sprake lijkt te zijn van een stromingsafhankelijke plaatjesfunctiestoornis, die niet opgepikt wordt door de standaard plaatjesfunctietesten.

In Hoofdstuk $\mathbf{5}$ werd een kosteneffectiviteitsanalyse gedaan van twee verschillende diagnostische algoritmes, in patiënten verwezen naar de hematoloog voor analyse bloedingsneiging. Het zorgverbruik, de kosten, de belasting voor de patiënt en de effectiviteit van de standaard stapsgewijze diagnostische benadering, werden vergeleken met een nieuwe 'alles in een' benadering, waarbij bijna alle beschikbare hemostasetesten in een keer gedaan werden. De resultaten toonden aan dat deze 'alles in een' benadering effectiever is en zorgt voor minder belasting voor de patiënt, maar dat deze benadering wel duurder is.

In Hoofdstuk 6 werd de bloedplaatjesfunctie van patiënten met door chemotherapie geïnduceerde trombocytopenie (CIT) onderzocht. De resultaten lieten zien dat er in deze bloedplaatjes sprake is van verstoring van verschillende processen met als voornaamste bevinding defecte receptor signalering ten gevolge van mitochondriële disfunctie, onafhankelijk van klassieke apoptose.

Hoofdstuk 7 beschrijft het onderzoek naar stolselafbraak bij CIT patiënten, onderzocht met de tPA-ROTEM, en het effect van bloedplaatjestransfusie hierop. Er werd aangetoond dat CIT patiënten zwakkere stolsels hebben die gevoeliger zijn voor stolselafbraak in vergelijking met gezonde personen. In een deel van de patiënten, maar niet bij iedereen, werd het stolsel minder gevoelig voor afbraak na het geven van een bloedplaatjestransfusie. Naast bloedplaatjes zijn er daarom waarschijnlijk andere factoren die de gevoeligheid voor stolselafbraak in CIT patiënten beïnvloeden. 
In Hoofdstuk 8 werden de belangrijkste bevindingen van dit proefschrift samengevat, en werden er een aantal stellingen gevormd en bediscussieerd. Ook werden onze toekomstplannen uiteen gezet. 


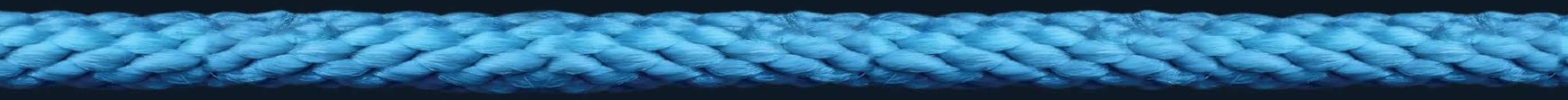


ADDENDUM

Impact paragraph 



\section{Impact paragraph}

Patients with mild bleeding disorders suffer from disproportionate bleeding after minor injuries, surgery or delivery, leading to use of blood products, re-surgery or prolonged hospitalization ${ }^{1,2}$. In daily life, bleeding symptoms like epistaxis or heavy menstrual bleeding, can have a great impact on social and school or work-related activities and can lead to lower quality of life $\mathrm{e}^{3-6}$. As pointed out in the preceding chapters, recognition and correct identification of mild bleeding disorders and establishing a personalized treatment plan, are crucial to prevent bleeding and its consequences, with accompanied economic and social benefits. However, in about $50-75 \%$ of the patients referred for bleeding evaluation, no reproducible abnormality in hemostatic laboratory tests is found ${ }^{7-9}$. In patients with bleeding of unknown cause (BUC), providing a personalized treatment plan ensuring save hemostasis for surgery or childbirth, and family counseling is difficult ${ }^{10}$.

Patients with a hematologic malignancy often develop low platelet count due to their disease or its treatment. A prophylactic transfusion strategy does not prevent bleeding in all patients on the one hand, and is, on the other hand not always necessary ${ }^{11,12}$. A personalized transfusion strategy can prevent unwanted complications and can save costs ${ }^{13}$.

The main goal of the studies described in this thesis is to improve diagnostic workup in patients with a bleeding tendency, to determine the value and place of new diagnostic tests, and to gain more insight in the mechanism of bleeding in different patient populations. The results of this thesis are not only relevant for scientific purposes, but are also of importance in social and economic perspective. The knowledge gained in the preceding chapters of this thesis (i) enables critical appraisal and use of established diagnostic tests and diagnostic approaches and, (ii) reveals potential pathophysiological mechanisms of bleeding in patients with bleeding of unknown cause (PFA-only) and patients with chemotherapy induced thrombocytopenia (CIT).

\section{Critical appraisal and use of established diagnostic tests and current diagnostic approaches in bleeding evaluation}

The studies described in Chapter 2, 3 and 5 investigated widely used and accepted diagnostic tests and approaches in bleeding evaluation.

Chapter 2 revealed that the bleeding assessment tool (BAT) alone is of limited value in the diagnostic work-up of patients referred for bleeding evaluation. A negative BAT was not able to exclude a mild bleeding disorder and a positive BAT increased the likelihood of having a mild bleeding disorder. However, the value of this 
increased likelihood in a referred setting is questionable. We believe that the main benefit of the BAT is that it provides a structured and complete interview of the patients' bleeding history. Chapter 3 showed that both Multiplate and platelet function analyzer (PFA) were not suitable as screening tests for mild platelet function disorders in preoperative patients and patient referred to the hematologist for bleeding evaluation. Although the results of both studies might be regarded as 'negative' findings, and might not lead to significant changes in guidelines, they do have the potential to increase awareness of hematologists and other physicians treating patients with bleeding symptoms, leading to well considered use of the BAT, Multiplate and PFA.

In Chapter 5 effectiveness, healthcare resource use and costs, and patient burden of two diagnostic approaches for patients referred for bleeding evaluation were compared. The newly proposed all-in-one diagnostic work-up was more effective and reduced patient burden, compared to the conventional stepwise approach, however this was at a higher cost. These findings open the discussion to what a conclusive diagnosis of a mild bleeding disorder and lower patient burden in the diagnostic process is worth. An important benefit of extensive bleeding evaluation, is the reassurance of complete investigation (even if no diagnosis is found) and the ability of putting together all the available pieces of evidence in order to carefully judge on the likelihood of having, or not having, a bleeding disorder.

\section{Potential pathophysiological mechanisms of bleeding}

In Chapter 4, 6 and 7, new experimental tests were evaluated in patients with bleeding of unknown cause (e.g. PFA-only patients) and patients with chemotherapy induced thrombocytopenia (CIT), in order to explore different mechanisms of bleeding. Our data adds new information to the recognized knowledge gaps of bleeding mechanisms in these patients. This will aid both physicians treating patients with congenital bleeding disorders and physicians treating patients with hematologic malignancies.

Chapter 4 described multiparameter microfluidic platelet function analysis in bleeding patients with a prolonged platelet function analyzer closure time as the only aberrant finding in their diagnostic work-up. Results showed altered microfluidic thrombus formation indicating a shear-dependent platelet function defect not detected by the static conventional hemostatic tests.

Chapter 6 described platelet activation processes and procoagulant activity in CIT patients and showed defective receptor signaling related to impaired mitochondrial function in platelets from these patients. In Chapter 7 the fibrinolytic potential of whole blood clots of CIT patients was explored with TPA-ROTEM, before and after 
platelet transfusion. Results showed that clots of CIT patients were more susceptible to tPA induced lysis compared to healthy individuals. Platelet transfusion resulted in less hyperfibrinolytic profiles, but not in all patients. Besides platelets, other factors are likely to influence clot lysis in CIT patients.

All studies are registered at the Dutch Trial Registry. Results described in this thesis have been, and will be, published in international peer review journals, and presented on (inter)national congresses. In this way, our results are shared with a broad audience of hematologists and other physicians treating bleeding patients and researchers in the field. We hope that in the end, we are able to change the statement 'we might never know why most patients bleed', into 'we might never know why some patients bleed'. 


\section{Reference}

1. Orsini S, et al. Bleeding risk of surgery and its prevention in patients with inherited platelet disorders. Haematologica 2017;102(7):1192-203.

2. Greaves M, Watson HG. Approach to the diagnosis and management of mild bleeding disorders. J Thromb Haemost 2007;5 Suppl 1:167-74.

3. Sanders YV, et al. Von Willebrand disease in the Netherlands: the WiN study. Ned Tijdschr Geneeskd 2014;158:A6518.

4. de Wee EM, et al. Health-related quality of life among adult patients with moderate and severe von Willebrand disease. J Thromb Haemost 2010;8(7):1492-9.

5. Blaauwgeers MW, et al. Congenital platelet disorders and health status-related quality of life. Res Pract Thromb Haemost 2020;4(1):100-5.

6. De Wee EM, et al. Impact of von Willebrand disease on health-related quality of life in a pediatric population. J Thromb Haemost 2011;9(3):502-9.

7. Quiroga $\mathrm{T}$, et al. High prevalence of bleeders of unknown cause among patients with inherited mucocutaneous bleeding. A prospective study of 280 patients and 299 controls. Haematologica 2007; 92(3):357-65.

8. Thomas W, Downes K, Desborough MJR. Bleeding of unknown cause and unclassified bleeding disorders; diagnosis, pathophysiology and management. Haemophilia, 2020.

9. Gebhart J, et al. High proportion of patients with bleeding of unknown cause in persons with a mildto-moderate bleeding tendency: Results from the Vienna Bleeding Biobank (VIBB). Haemophilia 2018; 24(3):405-13.

10. Rodeghiero F, et al. Fundamentals for a Systematic Approach to Mild and Moderate Inherited Bleeding Disorders: An EHA Consensus Report. HemaSphere 2019;3(5).

11. Wandt $\mathrm{H}$, et al. Therapeutic platelet transfusion versus routine prophylactic transfusion in patients with haematological malignancies: an open-label, multicentre, randomised study. Lancet 2012; 380(9850):1309-16.

12. Stanworth SJ, et al. A no-prophylaxis platelet-transfusion strategy for hematologic cancers. N Engl J Med 2013;368(19):1771-80.

13. Schiffer CA, et al. Platelet Transfusion for Patients With Cancer: American Society of Clinical Oncology Clinical Practice Guideline Update. J Clin Oncol 2018;36(3):283-99. 



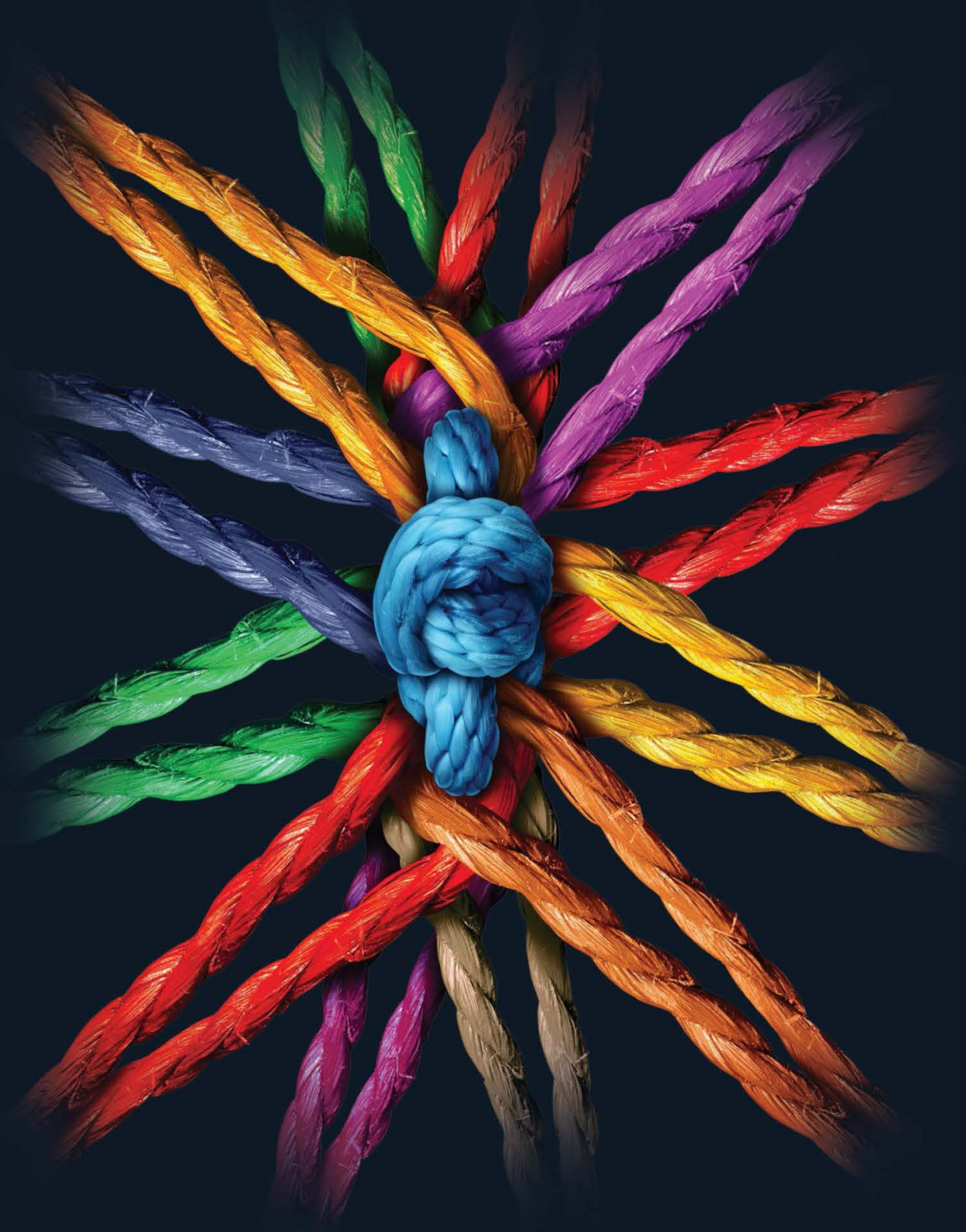


ADDENDUM

Dankwoord 

De afbeelding op de kaft van dit proefschrift staat voor verbinding.

Verbinding tussen de verschillende mechanismen om te komen tot een perfect stolsel.

Verbinding tussen de verschillende afdelingen, ieder met hun eigen expertise,

maar met een collectief belang: het verbeteren van patiëntenzorg.

Verbinding tussen mensen die vanuit hun ambities en talenten samen een doel realiseren. Vanuit gezamenlijke ambities en waarden. Vanuit echtheid en zichtbaarheid. Vanuit eigen verantwoordelijkheid en persoonlijk ondernemerschap. En vooral vanuit commitment.

Dan kan het bloed stromen (en stollen). Dan kan excelleren ontstaan.

Er zijn veel mensen die, op welke manier dan ook, hebben bijgedragen aan het maken van de verbindingen voor dit proefschrift en die mij gestimuleerd hebben om deze promotie tot een goed einde te brengen. Deze mensen wil ik hierbij persoonlijk bedanken.

Allereerst mijn promotoren, Prof. dr. Erik Beckers en Prof. dr. ir. Yvonne Henskens.

Beste Erik, in 2013 bespraken we voor het eerst de mogelijkheden tot het doen van onderzoek naar bloedingsziekten. Ik kwam toen vanuit een perifeer ziekenhuis en tijdens mijn opleiding tot hematoloog hebben we allebei lang volgehouden dat ik een echte 'perifere dokter' was. Maar... dat onderzoek, het academische, waarbij je dingen mag doen gewoon omdat je nieuwsgierig bent en omdat je er iets van wilt leren; het heeft me gegrepen. De eindeloze met-de-benen-op-tafel-plafond-staarsessies en de jaarlijkse congres lunch in de hoogste toren om de voortgang van mijn proefschrift te bespreken, dat zijn dingen die ik niet snel zal vergeten.

Lieve Yvonne, bedankt voor de fijne begeleiding tijdens mijn promotietraject. Jij bent voor mij het voorbeeld van hoe je op een warme en menselijke manier kunt excelleren in je vak. Door niet te zeuren, maar te zoeken naar oplossingen. Door soms iets te accepteren en los te laten, zodat je ook weer verder kunt. En door dicht bij jezelf te blijven. Bedankt voor je vertrouwen in mij als persoon. En voor de gezellige tripjes naar Cambridge!

Jullie manier van leiding geven is waar de afbeelding op de kaft van mijn boekje voor staat: verbinden. Daar blijf ik van leren.

Leden van de beoordelingscommissie, Prof. dr. H. ten Cate, Dr. J.M.E.M. Cosemans, Prof. dr. C.D. Dirksen, Prof. dr. M.P.M. de Maat, Prof. dr. J.J. Zwaginga, hartelijk dank voor het lezen en beoordelen van mijn proefschrift. 
Mijn dank gaat ook uit naar alle medeauteurs, voor het kritisch meedenken, het schrijven en het verbeteren van de artikelen. In het bijzonder wil ik bedanken $\mathrm{dr}$. Minka Vries, dr. Saskia Schols, dr. Patricia Nelemans en Prof. dr. Manuela Joore.

Lieve Minka, jouw precisie en vasthoudendheid waren voor mij een voorbeeld. Ik herinner me nog onze 'strijd' om een auteursplek. We gaven allebei niet toe, maar we konden er gewoon over praten. Dat is hoe het moet.

Beste Saskia, een groot deel van onze opleiding tot hematoloog hebben we samen gedaan. Onze gezamenlijke liefde voor stolling verbindt ons nog steeds.

Beste Patty, bedankt voor je epidemiologische en statistische adviezen, en zeker ook voor de interessante discussies over de verschillen tussen dokters en epidemiologen.

Beste Manuela, door jou ben ik op een andere, voor mij nieuwe, manier naar de gezondheidszorg gaan kijken. Bedankt voor jouw openheid en laagdrempeligheid. Ik hoop dat we deze eerste stap mogen uitbreiden tot iets moois.

De 'plaatjesclub' van de afdeling biochemie: Linda Herfs, Natalie Jooss, dr. Sanne Brouns, dr. Frauke Swieringa, dr. Constance Baaten, dr. Paola van der Meijden en Prof. dr. Johan Heemskerk. Jullie weten tot in de details hoe bloedplaatjes functioneren en ontwikkelen mooie manieren om deze details in kaart te brengen. Voor mij als dokter heel leerzaam. Bedankt voor deze samenwerking.

I also collaborated with several authors from the University of Cambridge in the United Kingdom: Prof. dr. Willem Ouwehand, dr. Kate Downes, dr. Karyn Megy. Thank you for the pleasant collaboration and teaching me about the do's and don'ts in genetics, for the diagnosis of patients with bleeding disorders.

De collegae van de Speciële Hemostase van het MUMC+, in het bijzonder: Paul Verhezen, Rick Wetzels, Rene van Oerle, Dave Hellenbrand en Ron Straat. Wat fijn om met jullie samen te werken! Jullie hebben het leeuwendeel van het laboratoriumwerk voor deze studies gedaan. En ook al hebben jullie het mega druk, er is altijd tijd om te overleggen, iets ingewikkelds te plannen, data ergens vandaan te toveren, last minute metingen te doen etc. Het is mooi om te zien met wat voor enthousiasme en kennis jullie je werk doen.

Met veel plezier heb ik studenten begeleid die tijdens, en sommigen zelfs na, hun wetenschapsparticipatie stage een bijdrage hebben geleverd aan de studies waarop dit proefschrift is gebaseerd. Paul Heuvelmans, Inge Verheijen, Edmée Kerkhofs, Lotte Smals, Lieve Faessen, Anita Engels, Lara Boerenkamp, Cristina Saelmans, Caspar Hullemans, Felicia Tjahjadi, Loes Ansems en Loïs Brady: bedankt voor jullie inzet, enthousiasme en gezelligheid. 
Mijn lieve kamergenoten: Marjolein van der Poel, Fabienne Magdelijns en Hanneke Joosten. Op een gezamenlijke werkplek deel je lief en leed met elkaar: slapeloze nachten, eerste stapjes, kolf ellende, contractperikelen, COVID drukte, huizenjacht, patiëntenzorg en noem maar op. Maar natuurlijk ook heel veel gezelligheid! Ik wil jullie bedanken voor al jullie steun. Marjolein en Fabienne, ik ben er trots op dat jullie mijn paranimfen willen zijn, nu komt het helemaal goed! Zodra het weer kan gaan we echt ons kamer etentje plannen!

Mijn directe collegae van de hematologie: Erik Beckers, Astrid Demandt, Michel van Gelder, Gwendolyn van Gorkom, Marjolein van der Poel, Janine van Elssen, Aniek de Coninck en Heidi van Ojik. Lieve collegae, bedankt voor de steun en de tijd die ik van jullie kreeg om te werken aan mijn proefschrift, zeker het laatste jaar. Vanaf nu zal ik weer meer zichtbaar zijn op de werkvloer, bij besprekingen en in de kliniek. We zijn een superfijn team. Bedankt daarvoor! En Janine, voor een diehard maligne hematoloog kun je de stolling prachtig illustreren, dankjewel!

Ons HBC-NEM team locatie Maastricht: Erik Beckers, Yvonne Henskens, Bernd Granzen, Paul Brons, Maroeska te Loo, Arian van der Veer, Sofie Reynders, Inge Merry, Joyce van Beers, Paul Verhezen, Dave Hellenbrand, Danielle Meijer, Tom van de Berg en Anne-Marije Hulshof. Bedankt voor de fijne samenwerking. En bedankt voor de fijne sfeer, laagdrempeligheid, humor, kennis en soms verhitte discussies tijdens ons wekelijks MDO op vrijdagochtend.

In het bijzonder wil ik nog benoemen de hemofilieverpleegkundigen, Inge Merry en wijlen Katrien van Rooij. Lieve Inge, door jouw harde werken heb ik de eindsprint in mijn promotietraject gehaald, bedankt daarvoor. We vormen een goed team en ik ben echt heel blij met jou als collega. En geloof me, onderzoek doen is echt heel leuk! Lieve Katrien, wat zou je dit geweldig gevonden hebben. Bedankt voor al jouw werk voor de 'bloody study' en je inzet voor onze patiënten.

Het HBC-NEM team van het Radboud UMC en het MMC-Veldhoven. Hoewel we elkaar de laatste tijd alleen maar digitaal gezien hebben, wil ik jullie bedanken voor de fijne samenwerking binnen de patiëntenzorg. Een etentje in Maastricht staat nog steeds op de planning.

Beste Tiny, bedankt voor het klaarmaken van dit proefschrift voor de beoordelingscommissie en je snelle reacties op mails als ik weer met wijzigingen kwam. Beste Jean, van een lief en vrouwelijk, naar een stoer en flitsend design. Bedankt voor al je werk en geduld! 
Zonder de steun van mijn familie en vrienden was het mij niet gelukt om dit proefschrift te schrijven naast mijn opleiding en baan.

Hartelijk dank aan ons vaste oppas team: Nicky, Zara en Jill. Wat zijn jullie toch lief voor Sophie en Luuk. Zo kon ik met een gerust hart gaan werken en heb ik ook in de weekenden meters kunnen maken!

Lieve pap en mam, dank jullie wel voor alle steun en vertrouwen in mij. Zonder jullie was ik nooit de persoon geworden die ik nu ben. Dankzij alle rondjes om de kerk, lukte het me ook om in de moeilijk momenten mezelf te blijven en mijn studie en later mijn opleiding, tot een goed einde te brengen. Jullie hebben altijd in me geloofd. Natuurlijk wil ik jullie ook bedanken voor alle oppasuren en logeerpartijtjes, voor de gezelligheid, maar ook als wij het even nodig hebben.

Lieve Charlotte en Isabelle, onverwacht kwam ik in jullie leven terecht. Dat was soms heel moeilijk, maar we hebben onze weg daarin gevonden. Wat ben ik trots op jullie! Charlotte, je werkt keihard om jouw pittige opleiding tot een goed einde te brengen. En met resultaat! Isabelle, ik bewonder jouw doorzettingsvermogen, ook in lastige situaties blijf je bij jezelf. Daar kunnen veel mensen een voorbeeld aan nemen. En dan jullie onvoorwaardelijke liefde voor Sophie en Luuk (en andersom!), zij hebben de beste grote zussen ever!

Lieve Sophie en Luuk, jullie zijn nog te klein om dit allemaal goed te begrijpen, maar weten ondertussen wel wat 'computeren' en 'mama moet nog even werken' betekent. Ik ben er trots op hoe jullie meegaan in ons hectische leven en vol goede moed de wereld tegemoet treden. Ik krijg zoveel energie van onze heerlijke knuffel momentjes. Word niet te snel groot!

Mijn allerliefste Patrick, woorden schieten te kort om jou te bedanken. Wat hebben we samen veel meegemaakt en bereikt de afgelopen 6,5 jaar. Heerlijke vakanties, ontspannen boottochtjes, een prachtig huis gekocht en twee fantastische kinderen rijker. Zeker het laatste jaar heb jij veel uren doorgebracht in de speeltuin, terwijl ik achter mijn computer zat. 'Ga maar naar boven, je moet het afmaken'. Zonder jouw steun en vertrouwen had ik dit niet kunnen doen. Ik ben er trots op hoe we deze tropenjaren samen managen. The sky is the limit: en dat nog neet! Ik hou van je. 



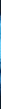


ADDENDUM

Curriculum vitae List of Publications 



\section{Curriculum vitae}

Floortje Catharina Josephine Irene Moenen werd geboren op 26 juni 1984 in Maastricht. In 2002 behaalde ze haar VWO diploma met het profiel 'natuur en gezondheid \& natuur en techniek' en muziek aan het Sint-Maartenscollege in Maastricht. Daarna startte zij aan de Universiteit Maastricht met de opleiding Geneeskunde. Tijdens deze opleiding liep ze coschappen in onder andere Ghana en Zuid-Afrika en deed ze haar wetenschapsparticipatie stage in Bergamo, Italië. In 2008 behaalde zij haar basisartsgetuigschrift en koos ze voor verdere specialisatie in de Interne Geneeskunde. Het eerste deel van haar opleiding vond plaats in het Catharina Ziekenhuis in Eindhoven, waarna ze in 2013 terug verhuisde naar Maastricht, voor de verdere specialisatie tot hematoloog aan het Maastricht Universitair Medisch Centrum+ (MUMC+).

Tijdens haar specialisatie startte ze haar promotieonderzoek binnen het project 'Predictors of Bleeding', onder supervisie van Prof. dr. E.A.M. Beckers, Prof. dr. ir. Y.M.C Henskens en Prof. dr. H.C. Schouten. Dit onderzoeksproject is een samenwerking tussen de klinische afdeling Hematologie/Interne Geneeskunde, het laboratorium voor Transfusie en Hemostase en de facultaire afdeling Biochemie binnen de researchschool Cardiovascular Research Institute Maastricht (CARIM). In 2014 onderbrak ze haar opleiding voor een jaar om de verschillende onderzoekslijnen binnen dit project op te zetten. Tijdens haar promotietraject publiceerde ze haar data in gerenommeerde internationale peer-reviewed medische tijdschriften en presenteerde zij haar data op diverse (internationale) conferenties. Daarnaast was zij tijdens haar opleiding bestuurslid en voorzitter van de junior afdeling van de Nederlandse Vereniging voor Hematologie.

In 2017 studeerde ze af als internist-hematoloog en kreeg zij een vaste aanstelling als hematoloog en hemofilie behandelaar in het MUMC+. In haar dagelijks werk ligt haar focus op het diagnosticeren en behandelen van patiënten met een bloedingsziekte en patiënten met myeloproliferatieve aandoeningen, paroxysmale nachtelijke hemoglobinurie en aplastische anemie. $\mathrm{Zij}$ is de lokale principal investigator van diverse (inter)nationale studies met betrekking tot deze ziektebeelden. Sinds 2021 is zij lid van de hoofdredactieraad van het Nederlands Tijdschrift voor Hematologie. Naast patiëntenzorg en research is zij actief binnen het onderwijs als docent voor o.a. de jaarlijkse transfusie en hemostasecursus, onderwijsdagen binnen de opleiding klinische chemie en de NVTH PhD cursus. Daarnaast is zij mentor van studenten in de bachelor geneeskunde en begeleidt zij meerdere master geneeskunde studenten tijdens hun wetenschapsparticipatiestage. Met haar werkzaamheden onderstreept ze het belang van een goede en sterke 
samenwerking en verbinding tussen clinici, laboratoria en onderzoeksafdelingen als basis voor innovatieve en excellente patiëntenzorg.

In haar vrije tijd is zij als arts en teamleider medische dienst verbonden aan het Huis voor de Pelgrim. In 2017 trouwde ze met Patrick Heubel, in 2018 werd hun gezin uitgebreid met dochter Sophie en in 2019 kregen zij zoon Luuk. 


\section{List of publications}

\section{Publications in international journal (Wi-1)}

Herfs L, Swieringa F, Jooss N, Kozlowski M, Heubel-Moenen F, van Oerle R, et al. Multiparameter microfluidics assay of thrombus formation reveals increased sensitivity to contraction and antiplatelet agents at physiological temperature. Thromb Res. 2021;203:46-56.

Perrella G, Huang J, Provenzale I, Swieringa F, Heubel-Moenen F, Farndale RW, et al. Nonredundant Roles of Platelet Glycoprotein VI and Integrin $\alpha$ llb $\beta 3$ in Fibrin-Mediated Microthrombus Formation. Arterioscler Thromb Vasc Biol. 2021;41(2):e97-e111.

van Lier YF, Davids M, Haverkate NJE, de Groot PF, Donker ML, Meijer E, FCJI HeubelMoenen, et al. Donor fecal microbiota transplantation ameliorates intestinal graftversus-host disease in allogeneic hematopoietic cell transplant recipients. Sci Transl Med. 2020;12(556).

Heubel-Moenen FCI, Henskens YMC, Verhezen PWM, Wetzels RJH, Schouten HC, Beckers EAM. Fibrinolysis in patients with chemotherapy-induced thrombocytopenia and the effect of platelet transfusion. J Thromb Haemost. 2019;17(7):1073-84.

Moenen FCJ, Vries MJA, Nelemans PJ, van Rooy KJM, Vranken J, Verhezen PWM, et al. Screening for platelet function disorders with Multiplate and platelet function analyzer. Platelets. 2019;30(1):81-7.

Baaten C, Moenen FCJI, Henskens YMC, Swieringa F, Wetzels RJH, van Oerle R, et al. Impaired mitochondrial activity explains platelet dysfunction in thrombocytopenic cancer patients undergoing chemotherapy. Haematologica. 2018;103(9):1557-67.

Moenen FCJ, Nelemans PJ, Schols SEM, Schouten HC, Henskens YMC, Beckers EAM. The diagnostic accuracy of bleeding assessment tools for the identification of patients with mild bleeding disorders: A systematic review. Haemophilia. 2018;24(4):525-35.

Moenen FC, Bakers FC, Bos GM. Pancreatic atrophy after allogeneic peripheral blood stem cell transplantation. Br J Haematol. 2016;172(2):155.

Moenen FC, van den Haak A, Gilissen LP. Colonic stenting for malignant bowel obstruction: cure or cause? Dig Liver Dis. 2011;43(5):416. 


\section{Publications in national journal (WN)}

Hulshof A, Heubel-Moenen FCI, Beckers EAM, ten Cate $H$, Henskens YM. Het geluid van hemostase: een nieuwe visco-elastische meetmethode met mogelijke toepassingen in transfusiemanagement, hypercoagulabiliteit en trombocytenfunctie. Ned Tijdschr Hematol. 2020;17:330-6.

Vries MJA, Heubel-Moenen FCJ, Henskens YM, Beckers EAM. De (on)zin van preoperatief screenen naar bloedingsziekten. Ned Tijdschr Hematol. 2019;16:151-6.

Moenen FCI, Rippen AW, Schouten HC. Arbeidsmarkt hematologen 2016. Ned Tijdschr Hematol. 2017;14:156-8.

Moenen FCJ, Vries MJA, Henskens YM, Cate ten H, Wetzels RJH, Verhezen PWM, et al. Uitdagingen bij het screenen en diagnosticeren van milde bloedingsziekten. Ned Tijdschr Hematol. 2016;13:182-9.

\section{Accepted for publication}

Heubel-Moenen FCJ, Brouns SLN, Herfs L, Boerenkamp SL, Wetzels RJH, Verhezen PWM, Machiels P, Megy K, Downes K, Heemskerk JWM, Beckers EAM, Henskens YMC. Multiparameter platelet function analysis of bleeding patients with a prolonged platelet function analyzer closure time. $\mathrm{Br} J$ Haematol.

\section{Submitted for publication}

Heubel-Moenen FCJ, Verhezen PWM, Wetzels RJH, van Oerle R, Straat R, Megy K, Downes K, Henskens YMC, Beckers EAM, Joore MA. Effectiveness and costs of a stepwise versus an all-in-one approach to diagnose mild bleeding disorders.

Kranen-Mastenbroek VHJM, Willems PCPH, Verhezen PWM, Beckers EAM, HeubelMoenen FCI, Henskens YMC. Need for preoperative hemostatic assessment in patients using valproic acid.

Goedhart MCHJ, Bukkems LH, Coppens M, Fijnvandraat CJ, Schols SEM, Schutgens REG, Eikenboom HCJ, Heubel-Moenen FCJ, Ypma PF, Nieuwenhuizen L, Meijer K, Leebeek WWG, Mathôt RAA, Cnossen MH, for the OPTI-CLOT study group and SYMPHONY consortium. Design of a prospective study on pharmacokinetic-guided dosing of prophylaxis factor replacement in hemophilia A and B (OPTI-CLOT TARGET study). 


\section{Oral presentations}

DHC 2020 - Fibrinolysis in patients with chemotherapy induced thrombocytopenia and the effect of platelet transfusion (Dutch Top Publications)

DHC 2020 - Multiparameter platelet function analysis of bleeding patients with a prolonged platelet function analyzer closure time

VHL/PAOKC symposium 2020 - Fibrinolysis in patients with chemotherapy induced thrombocytopenia and the effect of platelet transfusion

ISTH 2019 - Fibrinolysis in patients with chemotherapy induced thrombocytopenia and the effect of platelet transfusion

\section{Poster presentations}

ISTH 2021 - Cost-effectiveness of a stepwise versus an all-in-one approach to diagnose mild bleeding disorders (online poster pitch)

ISTH 2020 - Multiparameter platelet function analysis of bleeding patients with a prolonged platelet function analyzer closure time

EHA 2019 - Fibrinolysis in patients with chemotherapy induced thrombocytopenia and the effect of platelet transfusion

NVKC voorjaarscongres 2019 - Fibrinolysis in patients with chemotherapy induced thrombocytopenia and the effect of platelet transfusion (poster pitch)

NVB-TRIP symposium 2018 - Fibrinolysis in patients with chemotherapy induced thrombocytopenia and the effect of platelet transfusion

ISTH 2017 - Screening for platelet function disorders with the Multiplate and Platelet Function Analyzer

ISTH 2016 - Validation of a tissue plasminogen activator induced thromboelastometry in chemotherapy induced thrombocytopenic patients

ECTH 2016 - Validation of a tissue plasminogen activator induced thromboelastometry in chemotherapy induced thrombocytopenic patients

ISTH 2015 - Monitoring platelet transfusion in thrombocytopenic patients with hematologic malignancies measured by thromboelastometry

ISTH 2015 - Influence of pre-analytical heparin on t-PA induced fibrinolysis determined by thromboelastography despite the use of discard tubes

ASH 2015 - The diagnostic accuracy of bleeding assessment tools for the identification of patients with mild bleeding disorders: a systematic review 
